\author{
Aus dem Fachbereich Medizin \\ der Johann Wolfgang Goethe-Universität \\ Frankfurt am Main
}

\author{
betreut am \\ Zentrum der Pharmakologie \\ Institut für Klinische Pharmakologie \\ Direktor: Prof. Dr. Dr. Gerd Geißlinger
}

\title{
Screening for CEBPD-Modulating Compounds Using \\ a THP-1-Derived Reporter Cell Line in the Context of Rheumatoid Arthritis
}

\author{
Dissertation \\ zur Erlangung des Doktorgrades der theoretischen Medizin \\ des Fachbereichs Medizin \\ der Johann Wolfgang Goethe-Universität \\ Frankfurt am Main
}

vorgelegt von

Tatjana Ullmann

aus Orsk (Russland)

Frankfurt am Main, 2020 
Dekan:

Prof. Dr. Stefan Zeuzem

Referent:

Prof. Dr. Dr. Gerd Geißlinger

Korreferent:

Prof. Dr. Michael Parnham

Tag der mündlichen Prüfung: $\quad$ 28.04.2021 


\section{Content}

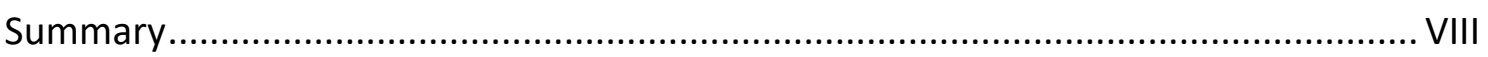

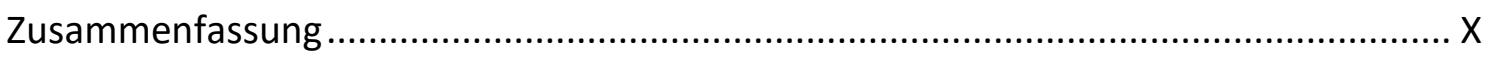

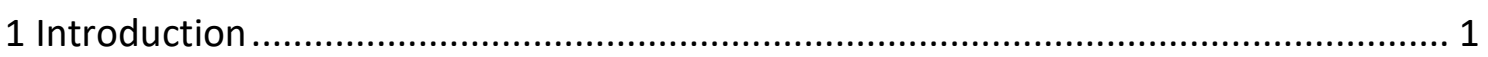

1.1 Phenotypic screening - a drug discovery strategy ............................................ 1

1.2 Gene reporters in phenotypic screening: SEAP and GLuc ................................ 3

1.3 Target gene for screening readout encodes C/EBPS ...................................... 5

1.3.1 Structure, regulation, and physiological function of C/EBP $\delta \ldots \ldots \ldots \ldots \ldots \ldots \ldots . . . . . . . . \ldots$

1.3.2 Role of C/EBP $\delta$ in the context of inflammation and related disorders ......... 15

1.3.3 Rheumatoid arthritis and the macrophage C/EBPS ................................ 18

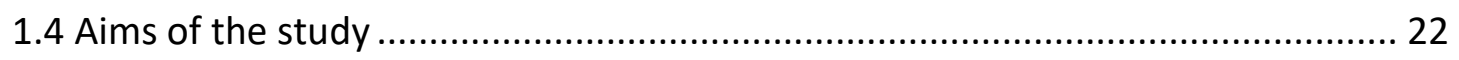

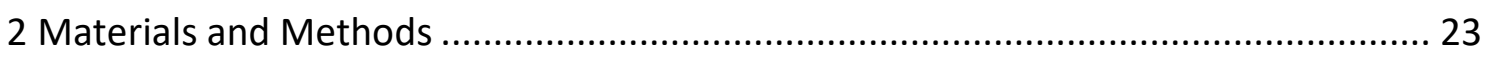

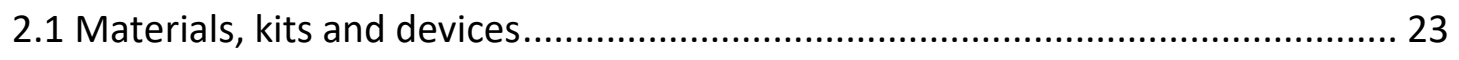

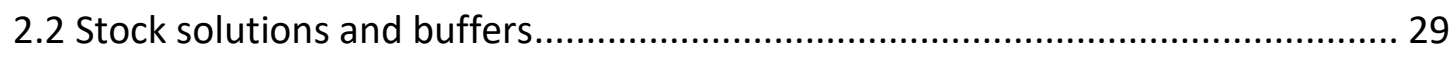

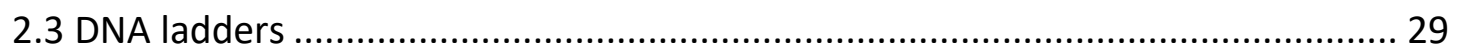

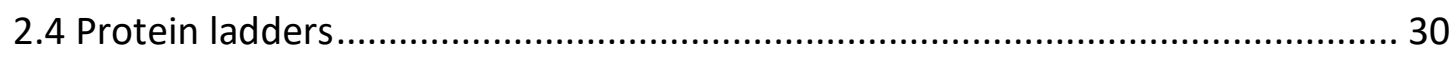

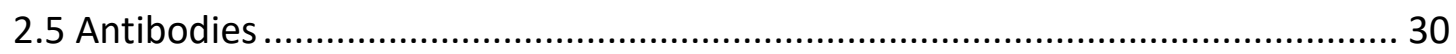

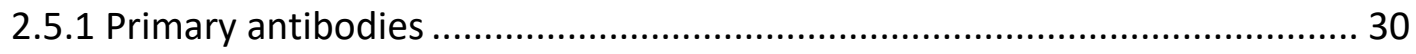

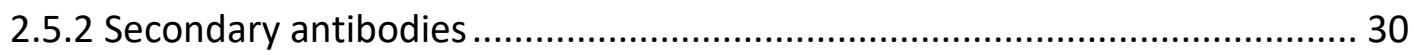

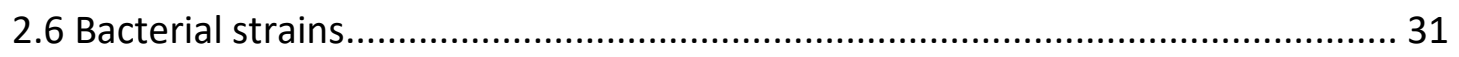

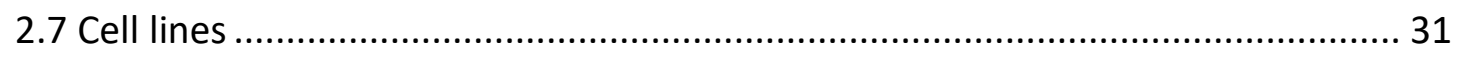

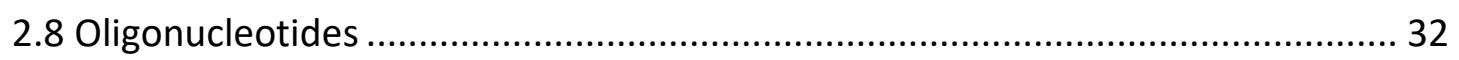

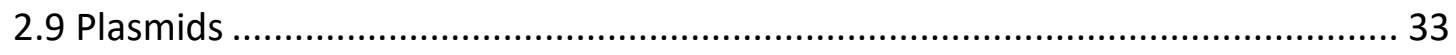

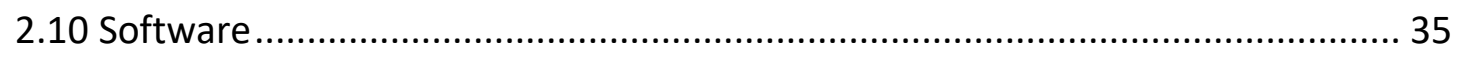

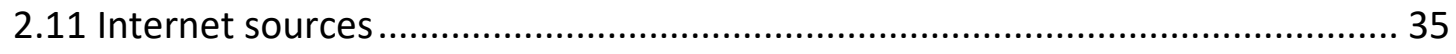

2.12 Determination of DNA and RNA concentration............................................ 37 


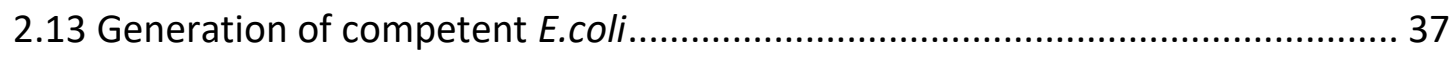

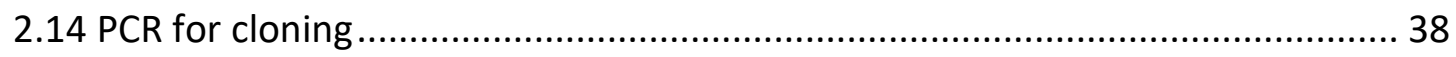

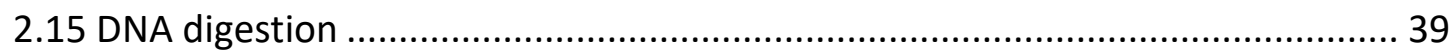

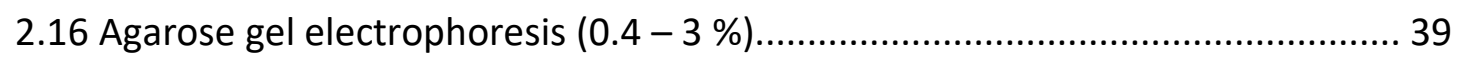

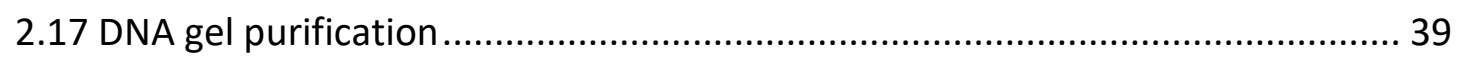

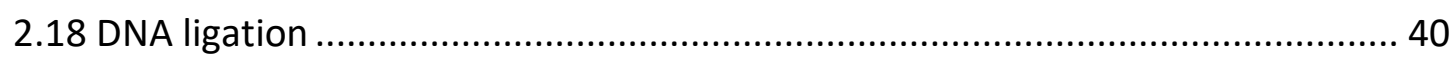

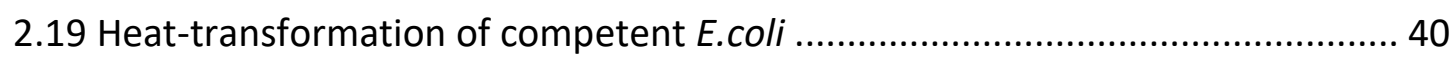

2.20 Plasmid preparation according to Holmes and Quigley ................................ 41

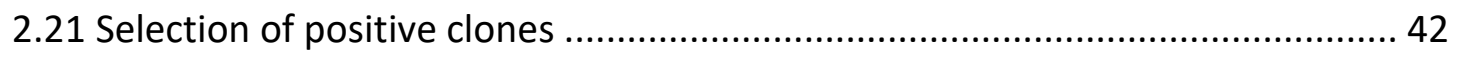

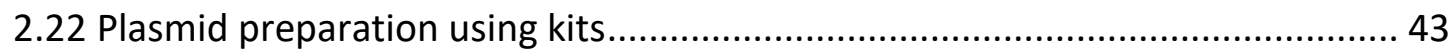

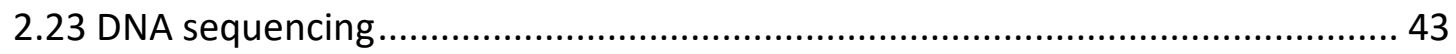

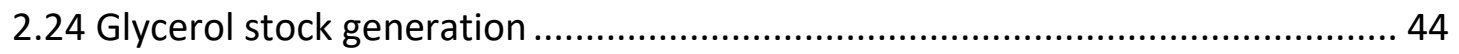

2.25 Bacterial artificial chromosome cloning ...................................................... 44

2.25.1 Verification of a wild type BAC clone ................................................ 44

2.25.2 BAC in vivo recombination (preliminary protocol) ................................. 45

2.25.3 Bacteria electroporation and positive clone selection (preliminary protocol)

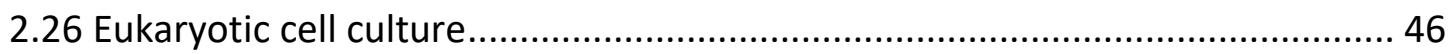

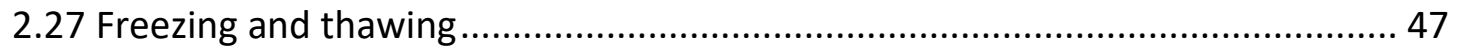

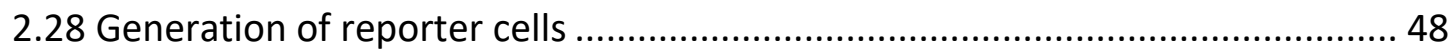

2.28.1 Transient and stabile transfection of HEK293T cells............................... 48

2.28.2 Generation of THP-1 reporter cell lines by viral transduction .................... 49

2.28.3 Generation of THP-1 reporter cells by electroporation (preliminary protocol) 50

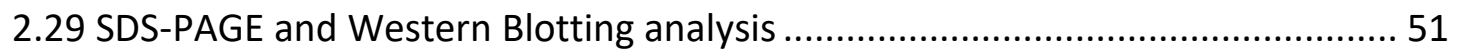

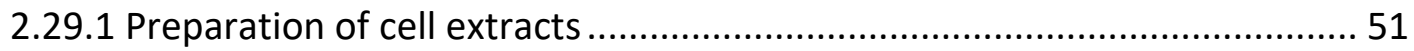


2.29.2 Determination of protein concentration ............................................ 51

2.29.3 SDS-polyacrylamide gel electrophoresis ............................................ 52

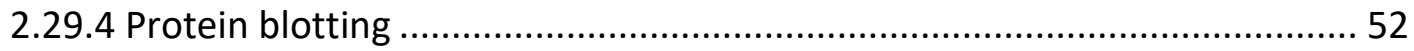

2.29 .5 Immunodetection of proteins ....................................................... 52

2.30 Preparation of immunofluorescence samples............................................. 53

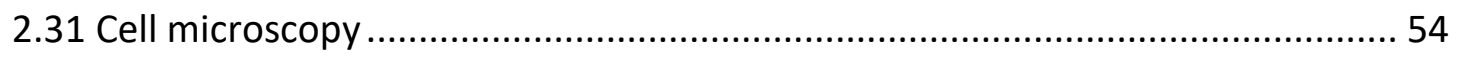

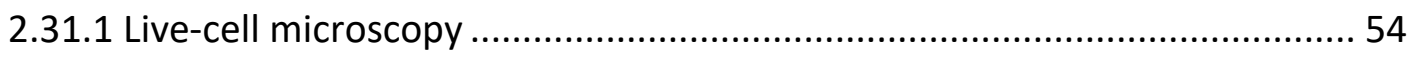

2.31.2 Confocal fluorescent microscopy .................................................... 54

2.32 RNA purification and real-time quantitative PCR .......................................... 54

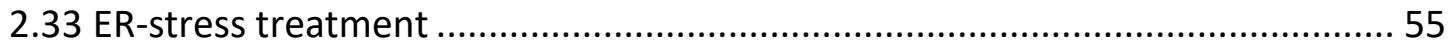

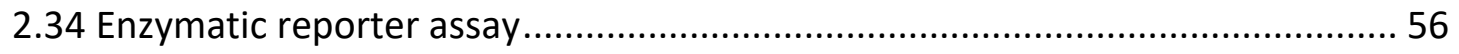

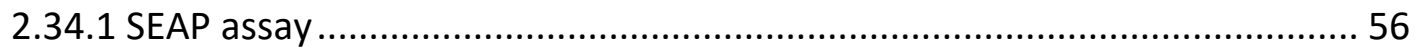

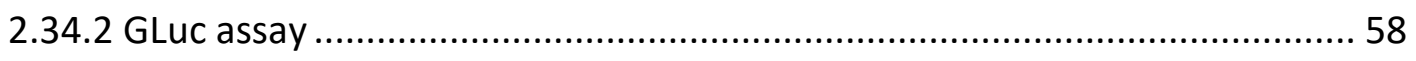

2.35 SEAP and GLuc assay validation parameters ............................................. 58

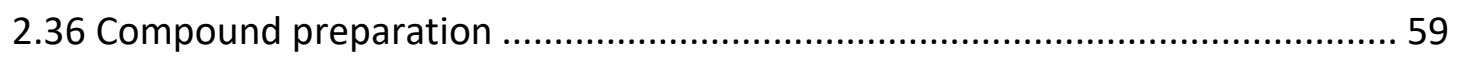

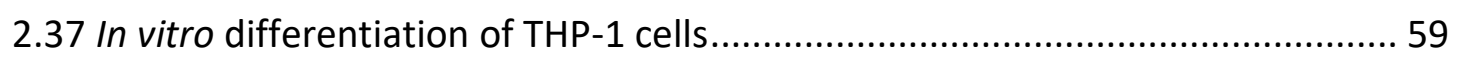

$2.38 \mathrm{M} 1$ treatment: in vitro polarization of THP-1 cells ......................................... 59

2.39 Screening for CEBPD-modulating compounds ........................................... 60

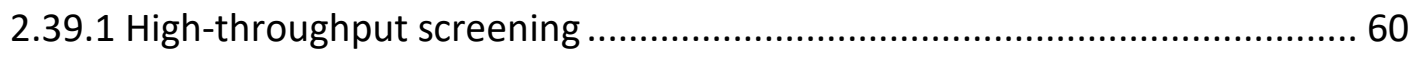

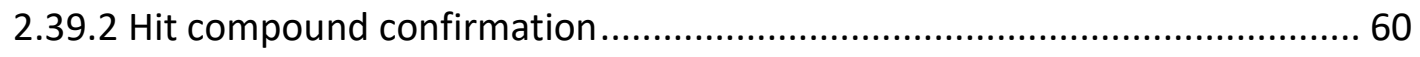

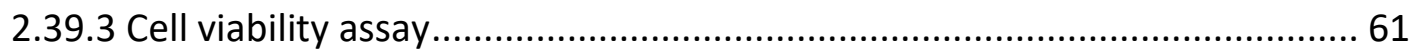

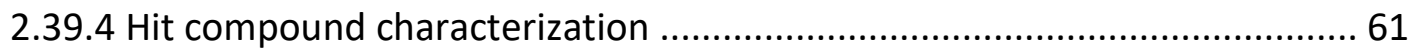

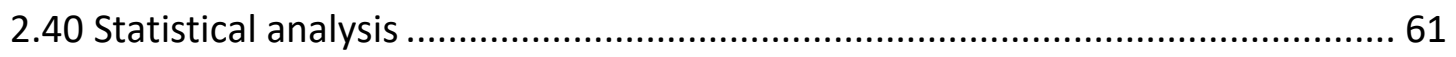

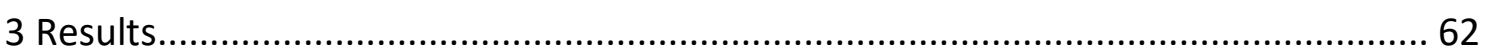

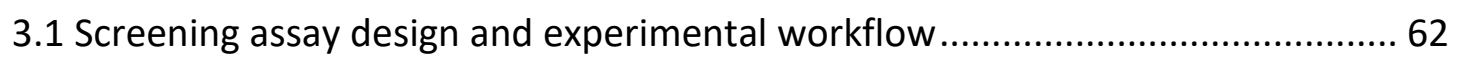

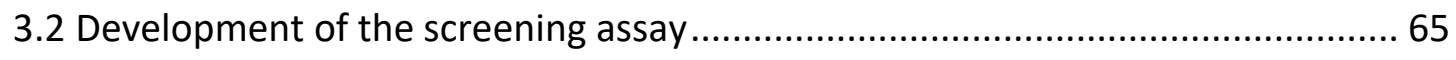


3.2.1 Generation and characterization of the multi-gene-reporter cassette $1.0 \ldots 65$

3.2.2 Generation and functionality test of the multi-gene-reporter cassette 2.0.. 72

3.2.3 Validation of the cellular system and specification of the screening procedure

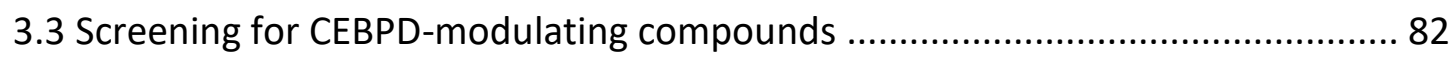

3.3.1 High-throughput screening of LOPAC ${ }^{\circledR 1280}$ and ENZO ${ }^{\circledR 774}$ libraries................ 82

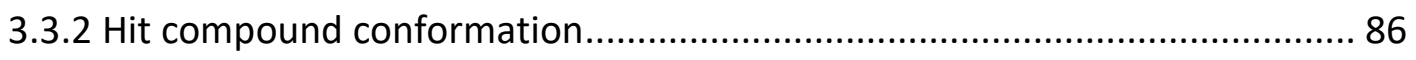

3.4 Epigenetically active hit compounds modulate CEBPD gene expression............. 89

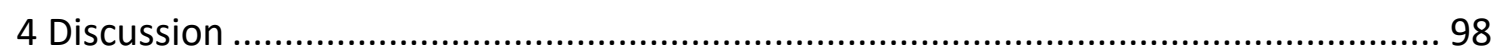

4.1 Translational potential of the developed screening assay ................................ 98

4.1.1 Phenotypic screening assay: evaluation of the chosen cellular system ........ 98

4.1.2 Phenotypic screening assay: evaluation of the chosen stimulus.................. 99

4.1.3 Phenotypic screening assay: evaluation of the chosen readout .................. 99

4.1.4 Evaluation of the screening tool: multi-gene-reporter cassette 1.0 .......... 100

4.2 Characterization of CEBPD-modulating hit compounds ................................ 102

4.2.1 Hit compounds and a general principle of their action ........................... 102

4.2.2 Hit compounds affect gene expression in THP-1 M

4.3 Epigenetic action of CEBPD-modulating hit compounds as RA treatment strategy

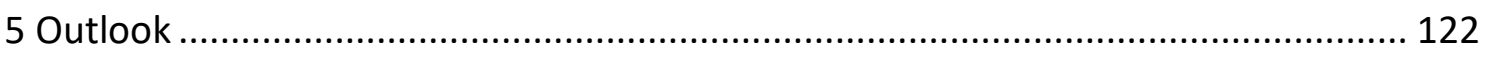

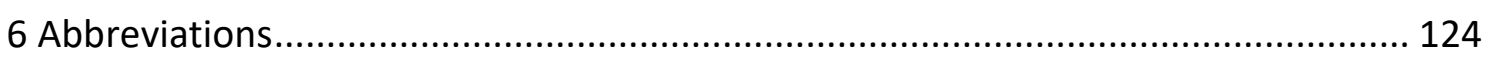

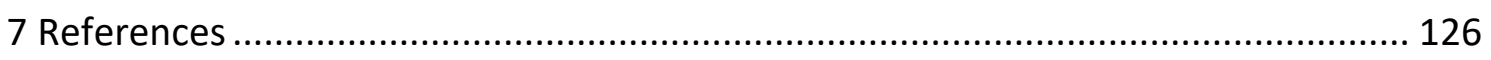

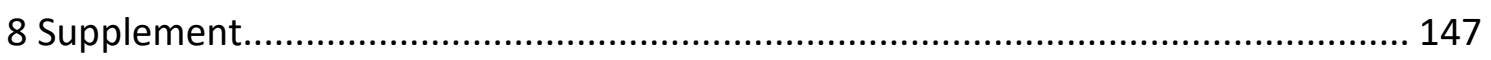

8.1 Structure and regulation of CEBPD target promoter .................................... 147

8.1.1 Sequencing of CEBPD-encoding region on BAC clone CH17-293N3 ............ 147

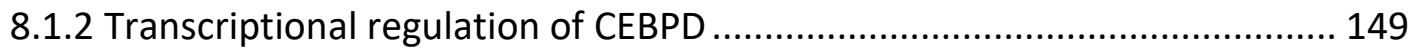


8.2 Characterization of SEAP and eGFP reporter assays ................................... 150

8.2.1 Characterization of colorimetric SEAP assay ........................................ 150

8.2.2 Characterization of fluorescent SEAP assay............................................ 151

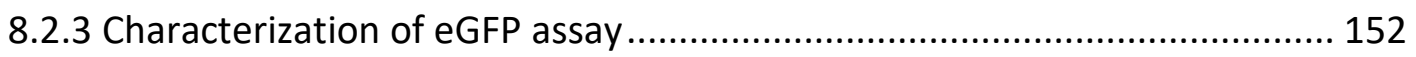

8.3 Characterization of CEBPD promoter strength ........................................... 154

8.4 Development of the final screening protocol ............................................... 156

8.4.1 Cell culture condition in 384 -well format ............................................... 156

8.4.2 Down-scaling and characterization of SEAP assay in 384-well format........ 158

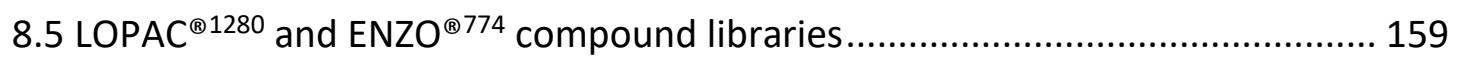

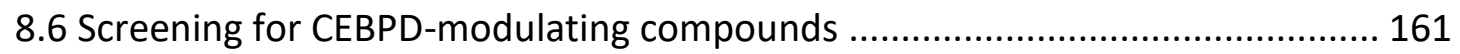

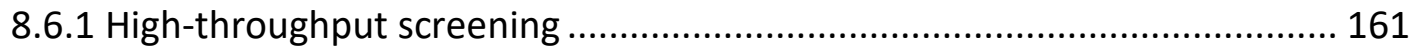

8.6.2 Hit compound confirmation .............................................................. 166

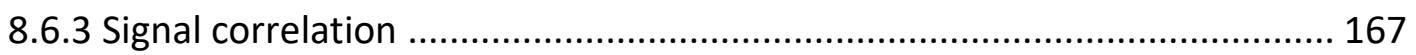

8.6.4 Hit compound characterization via RT-qPCR ........................................ 168

8.7 Analysis of TSA- and vorinostat-mediated effects in a longitudinal study ......... 170

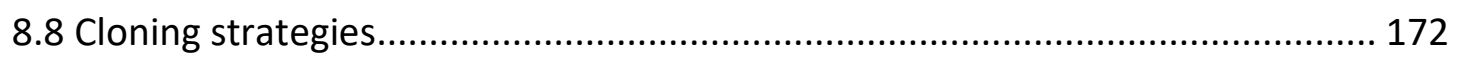

8.8.1 Cloning strategy of pcDNA3.1(-)-G2P-based vectors ............................. 172

8.8.2 Cloning strategy of SP-SEAP-myc-NLS-mCherry vectors .......................... 173

8.8.3 Cloning strategy of pSEW-eGFP-based viral vectors encoding SEAP and GLuc

8.8.4 Cloning strategy of the multi-gene-reporter cassette 2.0 in pcDNA3.1(-) backbone

8.8.5 Cloning strategy of multi-gene-reporter cassette 2.0 in pR6k backbone .... 179

8.8.6 Cloning strategy of multi-gene-reporter cassette 2.0 under control of CEBPD

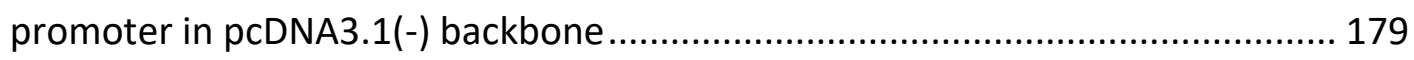

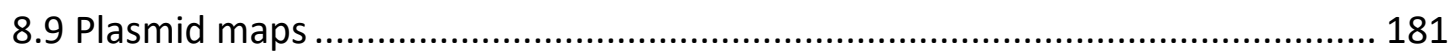


Content

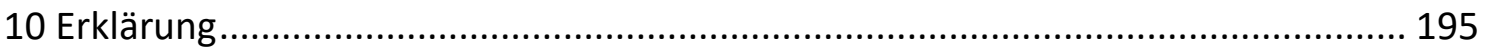




\section{Summary}

Development of treatment strategies of chronic inflammatory disorders relies on ongoing progress in drug discovery approaches and related molecular biologics. This study presents a gene reporter-based approach of phenotypic screening for anti-inflammatory compounds in the context of rheumatoid arthritis (RA).

CEBPD gene, used as the target gene for the screening readout, encodes CCAAT/enhancer binding protein delta (C/EBPS) transcription factor (TF). Structural and regulatory characteristics of CEBPD gene as well as function of C/EBPS TF in the context of inflammation satisfied assay requirements. C/EBPS TF acts as a key regulator of inflammatory gene transcription in macrophages $(\mathrm{M} \varphi)$ and is observed to contribute to disease development in both a rodent model of RA and RA patient biopsies.

Despite well-described pro-inflammatory effects of C/EBPS TF, it functions as a cell context-specific signal integrator showing also an anti-inflammatory activity. Consequently, both activation and inhibition of CEBPD alike may display a desired anti-inflammatory effect. The aim of this study was to develop a high-throughput screening assay for CEBPD-modulating compounds and confirm hit compounds' anti-inflammatory effects via gene expression analysis.

Generation and characterization of a multi-gene-reporter cassette 1.0 encoding enzymatic secreted alkaline phosphatase (SEAP) gene reporter was a priority during the assay development. Chemiluminescent SEAP assay demonstrating high assay sensitivity, broad linear range, high reproducibility and repeatability was chosen to monitor activity of the defined CEBPD promoter (CEBPD::SEAP). PMA-differentiated and M1-polarized THP-1-derived $\mathrm{M} \varphi$ stably expressing multi-gene-reporter cassette 1.0 were used as the assay's cellular system. mRNA expression of both reporter CEBPD::SEAP and endogenous CEBPD mirrored each other in response to a LPS and IFN-g-triggered inflammatory stimulus (M1 treatment), even though the defined CEBPD promoter region, utilized in the assay, contained only the most proximal and known regulatory sequences. SEAP chemiluminescence in the reporter cells' supernatant reliably correlated with the M1 treatment-induced CEBPD::SEAP gene expression. The final screening protocol was developed for semi-automatic screening in the 384-well format.

In total, 2054 compounds from LOPAC ${ }^{\circledR 1280}$ and ENZO ${ }^{\circledR 774}$ libraries were screened twice 
using the enzymatic SEAP readout with subsequent analysis of 18 selected compounds: nine with the highest and nine with the lowest signals, further characterized by qPCR. Gene expression levels of endogenous CEBPD, CEBPD::SEAP reporter as well as, IL-6, IL-1 $\beta$, and CCL2 as inflammatory markers were quantified. qPCR assays failed to correlate to SEAP readout in 15 compounds within three standard deviations (SDs) from solvent control: nine low signal and six high signal compounds. Demonstrating both assay sensitivity and specificity, a correlation between QPCR gene expression and SEAP readout was observed for three hit compounds with signals above three SDs: BET inhibitors (BETi) GSK 1210151A and Ro 11-1464 as well as an HDAC inhibitor (HDACi) vorinostat. The control compound trichostatin A (TSA) that reproducibly upregulated SEAP readout is also an HDAC inhibitor with a similar structure to vorinostat and was therefore included in the anti-inflammatory phenotype analysis.

The observed suppression of IL-6, IL-1ß, and CCL2 gene expression by hit compounds suggested their anti-inflammatory effect in THP-1 reporter M $\varphi$. mRNA expression of IL-6 and CCL2 was suppressed by HDACi and BETi at both 4 and 24 hours, while BETi reduced IL-1 $\beta$ mRNA expression 24 hour time point. BETi significantly upregulated gene expression of both reporter CEBPD::SEAP and endogenous CEBPD, 4 hours after M1 treatment. At the same time point, HDACi completely abolished the mRNA expression of the endogenous CEBPD, while simultaneously upregulating mRNA expression of the reporter CEBPD::SEAP. The use of the most proximal 300 base pairs region of endogenous CEBPD promoter, making the upstream regulatory elements unavailable in the assay, may account for differential expression levels of SEAP and C/EBPS TF. This observation corroborated the need to include a longer and more extensive CEBPD's gene regulatory area. Thus, an improved multi-gene-reporter cassette 2.0 was generated to be used on the basis of a bacterial artificial chromosome (BAC) covering CEBPD's genomic area of about 200,000 base pairs.

The generated screening assay is flexible, reliable, and sensitive displaying potential for drug discovery and drug repurposing. The pharmacological modulation of CEBPD gene expression, first reported for GSK 1210151A, Ro 11-1464, and vorinostat, contributes to the understanding of inflammatory responses in $M \varphi$ and may have RA therapeutic applications. 


\section{Zusammenfassung}

Die Entwicklung alternativer Behandlungsstrategien von chronisch-entzündlichen Erkrankungen baut auf dem kontinuierlichen Fortschritt in der Wirkstoffentdeckung und der damit verbundenen Molekularbiologie auf. Diese Arbeit präsentiert einen genreporterbasierten Ansatz eines phänotypischen Screenings zum Erkennen anti-entzündlich wirkender Verbindungen im Kontext von Rheumatoide Arthritis (RA).

Das CEBPD Gen, das für den CCAAT/enhancer binding protein delta (C/EBPS) Transkriptionsfaktor (TF) kodiert, stellte das Zielgen für den Screening-Readout dar. Strukturelle und regulatorische Charakteristika von CEBPD sowie die Rolle von C/EBPS TF im Entzündungsprozess erfüllten die Anforderungen des gewählten Screening-Ansatzes. C/EBPס TF fungiert als zentraler Regulator entzündlicher Gentranskription in Makrophagen $(\mathrm{M} \varphi)$ und wurde beobachtet, sowohl im RA-Tiermodell als auch in Gewebeproben von RA-Patienten, zur Krankheitsentwicklung beizutragen.

Trotz prominenter pro-entzündlicher Wirkung von C/EBPS TF, fungiert er als zellkontextspezifischer Signalintegrator, der auch eine anti-entzündliche Wirkung zeigt. Folglich können sowohl Aktivierung als auch Hemmung von CEBPD eine erwünschte anti-entzündliche Wirkung hervorrufen. Ziel dieser Arbeit war es, einen Hochdurchsatz-Screening-Assay für CEBPD-modulierende Verbindungen zu entwickeln und die entzündungshemmende Wirkung der Trefferverbindungen (Hits) durch Genexpressionsanalyse zu untersuchen.

Die Generierung und Charakterisierung der Multigenreporterkassette 1.0, die sezernierte alkalische Phosphatase (SEAP) als Genreporter exprimiert, war eine Schlüsselaufgabe bei der Assay-Entwicklung. Der chemilumineszente SEAP-Assay zeigte eine hohe Sensitivität, einen breiten linearen Bereich, sowie eine hohe Wiederholbarkeit und Reproduzierbarkeit und wurde daher zur Aktivitätsüberwachung des definierten CEBPDPromoters (CEBPD::SEAP) gewählt. PMA-differenzierte und M1-polarisierte THP-1 Reporter $M \varphi$, die die generierte Multigenreporterkassette 1.0 stabil exprimierten, stellten das zelluläre System des Assays dar. Die mRNA-Expression des CEBPD::SEAP Reporters und des endogenen CEBPD spiegelten sich in Reaktion auf die Behandlung mit LPS und IFN-g (M1-Behandlung), obwohl der definierte CEBPD-Promoter, der im Assay verwendet wurde, nur die proximalsten regulatorischen Sequenzen enthielt. SEAP zeigte die M1-behandlungsinduzierte CEBPD::SEAP Genexpression durch ihr erhöhtes Niveau im 
Überstand von THP-1 Reporterzellen zuverlässig an. Das endgültige Protokoll wurde für das halbautomatische Screening im 384-Well-Format entwickelt.

Insgesamt wurden 2054 Verbindungen von LOPAC $^{\circledR 1280}$ und ENZO ${ }^{\circledR 774}$ Bibliotheken mit Hilfe des enzymatischen SEAP-Readouts zweimal untersucht und anschließend 18 Verbindungen - neun mit dem höchsten und neun mit dem niedrigsten SEAP-Signal - mittels qPCR analysiert. Die Genexpressionsniveaus von endogenem CEBPD, CEBPD::SEAP Reporter sowie IL-6, IL-1ß und CCL2 Entzündungsmarker wurden quantifiziert. qPCR-Assays korrelierten nicht mit dem SEAP-Readout bei 15 Verbindungen innerhalb von drei Standardabweichungen (SDs) von der Lösungsmittelkontrolle: neun mit dem niedrigsten und sechs mit dem höchsten SEAP-Signal. Bei drei Hits mit SEAP-Signalen über drei SDs, den BET-Inhibitoren (BETi) GSK 1210151A und Ro 11-1464 sowie einem HDAC-Inhibitor (HDACi) Vorinostat, wurde eine Korrelation zwischen dem qPCR-Assay und dem SEAPReadout beobachtet, was sowohl die Assay-Spezifität als auch die Sensitivität zeigte. Die Kontrollverbindung Trichostatin A (TSA), die reproduzierbar ein hohes SEAP-Signal verursachte, gehört auch zu den HDACi und wurde daher in die Hit-Analyse mit einbezogen. Alle vier Hits zeigten ihre entzündungshemmende Wirkung durch die Suppression der Genexpression von IL-6, IL-1ß und CCL2. HDACi und BETi unterdrückten die mRNA-Expression von IL-6 und CCL2 4 und 24 Stunden nach der M1-Behandlung, während BETi die IL-1ß mRNA-Expression reduzierten. BETi regulierten Genexpression des CEBPD::SEAP und des endogenen CEBPD 4 Stunden nach der M1-Behandlung hoch. Zum gleichen Zeitpunkt, unterdrückten HDACi die mRNA-Expression des endogenen CEBPD, während sie diese des CEBPD::SEAP Reporters hochregulierten. Für die unterschiedlichen Expressionsniveaus von SEAP und C/EBPS TF kann die Verwendung der proximalsten 300 Basenpaare Region des CEBPD-Promoters verantwortlich sein, wodurch die distalen regulatorischen Elemente im Assay nicht verfügbar waren. Diese Beobachtung zeigte die Notwendigkeit, einen umfassenderen regulatorischen Bereich von CEBPD zu verwenden. Dafür wurde eine verbesserte Multigenreporterkassette 2.0 generiert, die auf einem bakteriellen künstlichen Chromosom (BAC) exprimiert werden sollte, das ein CEBPD-Genumfeld von 200.000 Basenpaaren umfasst.

Der entwickelte Screening-Assay ist flexibel, zuverlässig, sensibel und zeigt ein Potential für die Wirkstoffentdeckung. Die pharmakologische Modulation von CEBPD, erstmals gezeigt für BETi und Vorinostat, trägt zum Verständnis von Entzündungsreaktionen in $\mathrm{M} \varphi$ bei und weist mögliche therapeutische Anwendungen für RA auf. 


\section{Introduction}

\subsection{Phenotypic screening - a drug discovery strategy}

Process of drug discovery starts with basic biology research focusing on characterisation of original physiological and pathological signalling pathways, involved interactions, and molecular interlayers. Obtained evidence is further investigated during pre-clinical research using available or generating new cell-based or animal-based disease models. At this step, promising pharmacologically active compounds are identified and characterized. Their safety and efficacy is further evaluated during clinical studies performed on healthy volunteers and then on a targeted patient cohort. The most successful candidate with desired and proven functions is finally released as a novel drug available for patients.

In drug discovery process, translational research aims to integrate basic biology evidence into practical solutions. Therefore, translational research is considered to speed up scientific discovery into applied therapeutic uses for a patient benefit. ${ }^{1}$ Target-based or system-based (phenotypic) screening approaches are used in preclinical research to identify potential drug candidates. ${ }^{2}$

Phenotypic screening, which is defined as the testing of a large compound number in a system-based manner, ${ }^{3}$ is target-agnostic and focuses on monitoring of screening compound-mediated changes in cellular phenotype. ${ }^{4,5}$ In contrary, target-based screening relies on determined identity of target molecule and its molecular mechanism of action (MMOA). ${ }^{2}$ Here it is important to consider pharmacological plasticity of identified drug candidates, as a chosen target molecule is never static in an organism treated dynamically. ${ }^{6}$ Phenotypic screening advantageously enables identification of compounds that act through unknown targets or unexpected MMOA for known targets. ${ }^{2,4,5}$ In the period from 1999 to 2013, 33 of first-in-drugs approved by U.S. Food and Drug Administration (FDA) were discovered using a system-based approach and eight of them - by the phenotypic screening. ${ }^{3}$ Several drug candidates such as bromodomain inhibitors derived from a phenotypic screening. 3,7

Translational ability of phenotypic screening relies on a screening assay design defined through three specific criteria associated with chosen pathological disorder - stimulus, 
system, and readout (Fig.1-1). ${ }^{4}$ The chosen assay system is represented by a relevant (and best available) in vitro or in vivo disease model that displays major disease-related pathological aspects. ${ }^{4}$ Phenotypic screening is mostly performed in a high-throughput format using a well-established cell line. The chosen stimulus, applied during phenotypic screening assay, should activate a relevant network of signalling pathways that contribute to the targeted disease pathology. ${ }^{4}$ The chosen screening assay readout should display a close proximity to the clinical end point of selected disorder. ${ }^{4}$

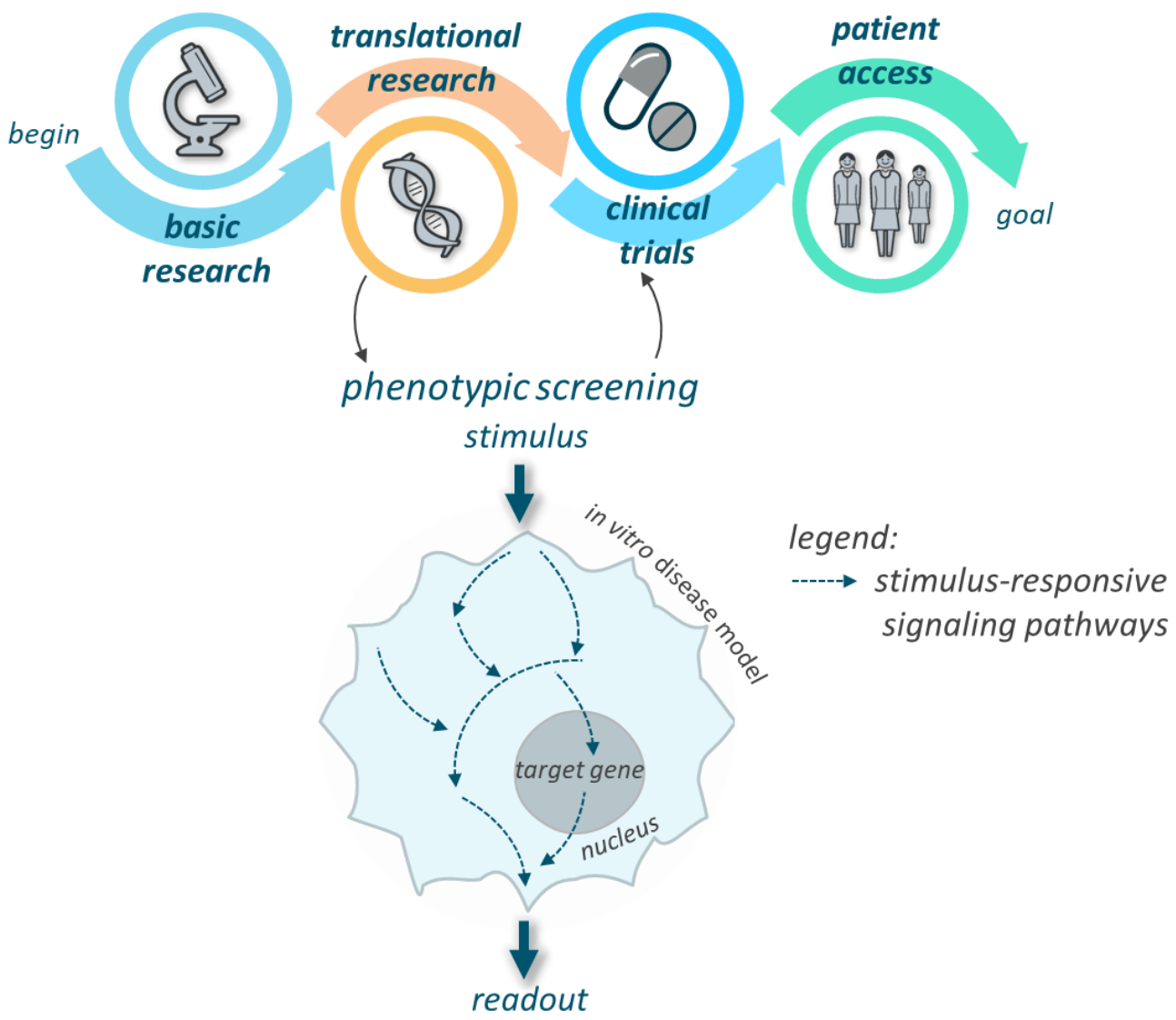

Figure 1-1: Overview of phenotypic screening characteristics in the context of a drug discovery pathway. Drug discovery is a complex process that undergoes several stages. It originates at the level of basic research, is directed toward clinical trials over translational research, and terminates at the desired stage of drug associability for patients. Being a translational research approach, phenotypic screening is performed in a target-agnostic manner and focuses on monitoring of compound-mediated changes in a cellular phenotype. In the chosen disease context, represented here by an in vitro disease model, the chosen stimulus activates corresponding known and unknown signalling pathways (arrows) leading to phenotypic alterations detected by the chosen readout. As cellular phenotype is also defined by the environment-responsive gene and protein expression, promoter activity monitoring of a carefully chosen target gene can be used as a screening readout.

During phenotypic screening, the choice of downstream readout beneficially enables observation of a larger number of mechanisms that modulate cellular phenotype in a chosen disease context. ${ }^{4}$ Here, monitoring of gene expression as an assay readout gains a special status relying on the connection of the gene function to a cellular phenotype. 
Accompanied by other factors, an environment-responsive gene and protein expression define cell's individual morphology and function resulting in a cell-specific phenotype. ${ }^{8}$ Gene reporter assays represent a specific category of phenotypic screening ${ }^{3}$ relying on the relationship between gene expression and the resulting cellular phenotype. For a reliable readout, this type of phenotypic screening requires careful selection of both target gene and gene reporter proteins.

\subsection{Gene reporters in phenotypic screening: SEAP and GLuc}

Gene reporter is a gene that is expressed under control of a chosen promoter and encodes an easily assayed protein. ${ }^{9,10}$ Reporter proteins reveal cellular processes in the cell uncharacterized otherwise. Gene reporter assays belong to molecular-biologic instruments widely-used to identify modulators of signal transduction and gene expression ${ }^{11-}$ 13 and are also suitable for the phenotypic screening for drug candidates. ${ }^{14,15}$ During phenotypic screening, stimulation of an upstream receptor-mediated or receptor-independent signalling activates transcription factors that bind a target promoter and activate a reporter gene. ${ }^{4}$ Thus, the screening-compound mediated activation of a target promoter is detected by measurement of reporter protein activity by the corresponding assay. To the common gene reporters used in phenotypic screening belong intracellular (e.g. chloramphenicol acetyltransferase, Firefly and Renilla luciferases, or fluorescent proteins) or extracellular (e.g. SEAP, secreted luciferases) proteins. ${ }^{14}$ In this study, multiple reporter proteins such as SEAP and GLuc were used.

Secreted alkaline phosphatase (SEAP), derived from the membrane-bound human placental alkaline phosphatase (PLAP), ${ }^{16}$ is a well-established gene reporter that is used for monitoring of gene expression, ${ }^{17}$ promoter activity, ${ }^{18}$ or endoplasmic reticulum (ER) stress. ${ }^{19}$ SEAP enzymatic activity can be detected in lysates or cellular supernatants of SEAP-expressing mammalian cells via colorimetric, fluorescent, or chemiluminescent assays. In absence of ER-stress affecting the secretory pathway, changes in SEAP levels in cellular supernatant are linear to changes in intracellular SEAP mRNA and cell number. ${ }^{16,20}$ SEAP displays a high protein stability with a half-live of over 20 days ${ }^{21}$ leading to its accumulation in cellular supernatant upon promoter activation and so correlates with bulk levels of target gene expression. SEAP is easy to distinguish from endogenous pro- 
teins as it is resistant to inhibition by L-homoarginine and shows high heat tolerance, in contrary to other phosphatases. ${ }^{16,22}$

Isolated from a marine copepod Gaussia princeps, Gaussia luciferase (GLuc) is one of the brightest and smallest luciferases ${ }^{23,24}$ and is used as a gene reporter for monitoring of gene expression, ${ }^{25}$ promoter activity, ${ }^{26}$ ER-stress, ${ }^{27}$ or protein-protein interaction. ${ }^{28} \mathrm{Be}$ ing a naturally secreted protein, ${ }^{23}$ GLuc can be detected in both lysates and cellular supernatants of GLuc-expressing mammalian cells. In the absence of ER-stress, changes in GLuc levels in conditioned medium are direct proportional to cell number, growth, and proliferation. ${ }^{27,29}$ GLuc oxidizes the natural substrate coelenteracine to coelenteramide 25,30 - a light-producing molecule, which bioluminescent signal rapidly decays after substrate addition. Chemiluminescent detection of GLuc requires, therefore, a luminometer with a built-in injector. GLuc itself shows high protein stability in the conditioned medium with a half-live of 6 days. ${ }^{31}$ As GLuc is a non-human protein, its detection in cellular supernatants of human target cells is not biased by the activity of endogenous proteins.

During phenotypic screening, the use of secreted enzymatic gene reporters like SEAP or GLuc display following advantages: i) no need for cell lysis, allowing cells to be used for further analysis; ii) temporally flexible detection of enzymatic activity in cellular supernatants; iii) possibility to perform longitudinal studies; iv) reduced assay variability and increased assay stability due to the long half-lives of reporters. Nonetheless, secreted reporter assays show following limitations during screening: i) no indication of transfection efficiency or cell viability for transient transfected reporter cells; ii) sensitivity to ER stress; iii) need to select distinct enzyme-specific substrates for the use of both enzymes in the screening assay. Gene reporter assays may display further limitation in screening reliability, as gene reporters are usually expressed under control of core promoters, which can lack regulatory sequences present in endogenous promoter of a target gene. 


\subsection{Target gene for screening readout encodes C/EBP $\delta$}

\subsubsection{Structure, regulation, and physiological function of C/EBP $\delta$}

\subsubsection{Structure of CEBPD gene, C/EBP $\delta$ mRNA, and C/EBP $\delta$ protein}

CCAAT/enhancer binding proteins (C/EBPs) belong to a family of transcription factors (TFs) of the basic-leucine zipper (bZIP) class. ${ }^{32}$ The name refers to the ability of the first identified C/EBP TF to bind CCAAT box of several promoters and core homology region of some virus enhancers. ${ }^{33}$ In mammalian cells, six members of C/EBP gene family were identified to date: C/EBP $\alpha, C / E B P \beta, C / E B P Y, C / E B P \delta, C / E B P \varepsilon$, and DDIT3 (CHOP, C/EBPZ), designated by Greek characters indicating the chronological order of their discovery. ${ }^{34}$

CCAAT/enhancer binding protein delta (C/EBPS, alternative names CELF, CRP3, NF-IL6 3 , RcC/EBP2), is discovered in 3T3-L1 adipoblast cells in an effort to identify regulatory protein factors in the context of adipocyte differentiation. ${ }^{35}$ CEBPD gene that encodes C/EBP $\delta$ protein displays a very simple gene structure containing a distinct promoter and a single exon. ${ }^{35}$ As CEBPD gene lacks introns, alternative promoters or alternative polyadenylation sites, ${ }^{35}$ a single C/EBP $\delta$ mRNA is produced. ${ }^{36}$ CEBPD gene, which is among the shortest human protein-coding genes (around 1.2 kilobases), is located on chromosome $8\left(8 p 11^{36}\right.$ or $\left.8 q 11.21: 47,738,164-47,736,913^{1 *}\right)$ (Fig.1-2). Homology analysis between human CEBPD and rodent cebpd genes reveals high similarity in protein coding and untranslated regions, less similarity in proximal promoter region, upstream of transcription start site (TSS), and no similarity in sequences downstream of transcription termination site. ${ }^{36}$

According to different sources, C/EBP $\delta$ mRNA contains $1269^{36}$ or $1252^{1 *}$ nucleotides showing differences in length of the 3 ' UTRs. In this study, I refer to genomic information for CEBPD gene (NC_000008.11), C/EBPS mRNA (NM_005195.4) and protein (NP_005186.2) provided by NCBI ${ }^{1 *}$ (Fig.1-2). High instability with a half-time of 35-40 minutes and relatively short poly-adenylation tail characterize human C/EBPS mRNA. ${ }^{36,37}$ Due to the lack of introns, C/EBPS mRNA cannot be exported from the nucleus by exon-junction complex, however, mechanisms of C/EBPS mRNA nuclear export

\footnotetext{
1* Gene 1052 (CEBPD, Homo sapiens). Bethesda MD: National Library of Medicine (US), National Center for Biotechnology Information; 2004. https://www.ncbi.nlm.nih.gov/gene/1052. Accessed September 10, 2019.
} 
Introduction

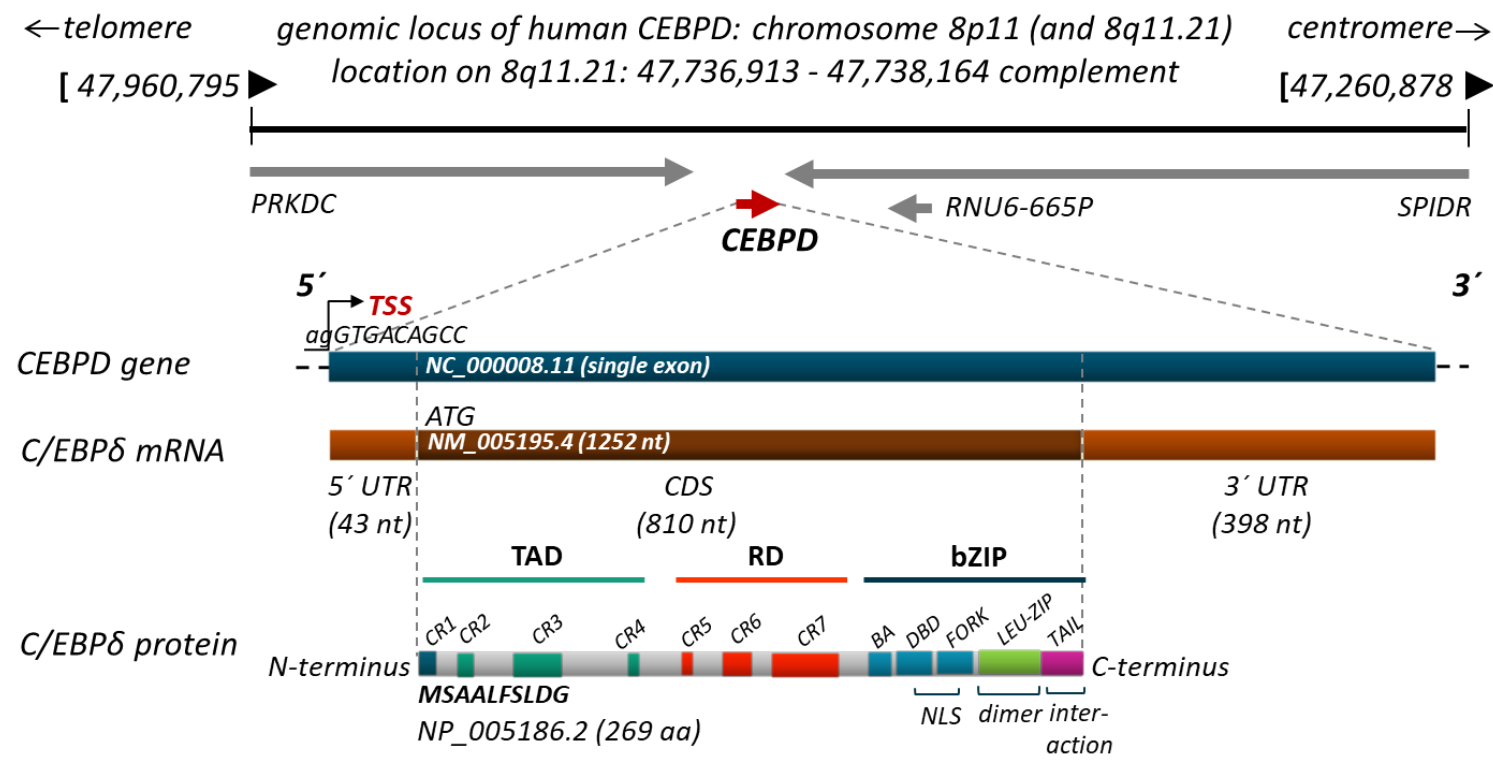

Figure 1-2: Genomic location and structure of human CEBPD gene, mRNA, and protein. Human CEBPD gene (red arrow) is encoded on chromosome 8, where its cytogenetic locus location is reported as both $8 \mathrm{p} 11$ and $8 \mathrm{q} 11.21$. On 8q11.21, CEBPD is encoded on a complementary strand at indicated positions, surrounded by SPIDR, RNU6-665P, and PRKDC neighbourhood genes (grey arrows). This schematic drawing is based on the GRCh38.p13 primary assembly (NCBI). ${ }^{1 *}$ CEBPD gene NC_000008.11 contains a distinct promoter and a single exon lacking introns, alternative promoters or polyadenylation sites. The single C/EBPS mRNA NM_005195.4 (1252 nt) is translated into a C/EBPS protein (NP_005186.2), of which only one full-length ( 269 amino acids, fist 10 are indicated in bold) functional protein isoform is known. C/EBP $\delta$ protein contains structural and functional domains including the highly conserved C-terminal basic leucine-zipper (bZIP) module, the regulatory domain (RD), and the variable $\mathrm{N}$-terminal trans-activation domain (TAD). bZIP contains TAIL sequence responsible for protein-protein interactions, the leucine zipper (LEU-ZIP) required for dimerization, DNA binding domain (DBD) and FORK, both encoding nuclear localization signal (NLS), and basic/acidic region (BA). TAD and RD contain C/EBP intra-family conserved regions (CR) CR-1 to CR-8 (blue, green, and red areas) that are connected by low conservation regions (grey areas). Individual functions of CRs are further described in text. TSS: transcription start site; ATG: translation start codon; UTR: untranslated region; CDS: coding sequences.

have not been described yet. ${ }^{36}$ Derived from the intronless CEBPD gene, C/EBP $\delta$ premRNA is not processed by the splicing machinery and is translated to a single C/EBP $\delta$ protein of approximately 28 kilo Daltons. ${ }^{36}$ To date, only one full-length (269 amino acids) functional C/EBP $\delta$ protein is known. ${ }^{36,38} \mathrm{C} / \mathrm{EBP} \delta$ protein displays a short biological half-life ranging between two and four hours in epithelial cells and macrophages. ${ }^{37,38}$

C/EBPS TF is a modular protein containing an assembly of structural and functional domains: C-terminal bZIP module, regulatory domain (RD), and $\mathrm{N}$-terminal transactivation domain (TAD) (Fig.1-2). The C-terminal amino acid sequence of bZIP is up to $90 \%$ identical in all C/EBP isoforms. ${ }^{33,36,39}$ The highly conserved bZIP module contains the pre-bZIP basic/acidic region (BA), the DNA binding domain (DBD), the fork sequence (FORK), the leucine zipper (LEU-ZIP) dimerization domain and a post-bZIP C-terminal peptide TAIL $^{36,40}$ (Fig.1-2). The BA, DBD and FORK regions of bZIP share their basic amino acid 
nature and are responsible for DNA binding capacity and nuclear transport of C/EBPS. As a structural part of C/EBPS TF, which directly interacts with DNA, DBD provides a cognate recognition of specific sequences in the promoter regions of C/EBP $\delta$ target genes. ${ }^{34,36}$ The positively churched basic-amino-acid-rich DBD binds to a negatively charged acidic DNA. ${ }^{41}$ Additionally, DBD, complemented by FORK sequence, serves as the primary nuclear localization signal (NLS) for bZIP factors, including C/EBPS. ${ }^{42}$ In a bZIP module of C/EBPS TF, leucine zipper is represented through a heptad of leucine repeats that intercalate with that of the dimer partner resulting in a formation of a coiled-coil of parallel oriented $\alpha$-helices. ${ }^{43}$ Thus, the LEU-ZIP is responsible for the formation of homo- and heterodimers with other transcription factors of C/EBP family and of a larger bZIP superfamily. ${ }^{34}$ The C-terminal peptide, TAIL, is identified in association with many bZIP modules and is characterized as an extension of the zipper dimerization domain. ${ }^{44}$ It is proposed to act as a protein-protein interaction motif for diverse associations. $^{45}$

The regulatory domain (RD) of C/EBPS TF serves as a platform for the most known and described post-translational modifications. Specific amino acids, their location, and a type of the post-translational modification of C/EBP $\delta$ are described later in this chapter.

The N-termini containing transactivation domain (TAD) are rather divergent with less than $20 \%$ sequence identity between C/EBP family members. ${ }^{36,46}$ The TAD contains effector sequences that are reported to be important for protein-protein regulatory interactions, which mediate C/EBP $\delta$ TF's trans-activation. ${ }^{34}$ Certain examples of such mediation are defined later in this chapter.

Sequence alignments of $C / E B P \alpha, C / E B P \beta, C / E B P \delta$, and $C / E B P \varepsilon$ proteins reveal a presence of short discretely conserved regions (CR, CR1 to CR7) in their regulatory and trans-activating domains ${ }^{36,40,47}$ (Fig.1-2). CR2, CR3, CR4, and CR6 reveal high conservation of primary structures even between species, whereas in CR5 and CR7 the similarities between C/EBPs were found more relaxed. ${ }^{40}$ The conserved C/EBP CRs are connected by a so called low complexity regions (LCR) displaying less degree on conservation. ${ }^{40} \mathrm{CR} 1$ to CR4 constitute the N-terminally TAD and CR5 to CR7 are located in RD. ${ }^{48}$ Although, CR1 is restricted to C/EBP 3 , sequence similarities are found in C/EBPS. ${ }^{40} C R 2, C R 3$, and CR4 are proposed to interact with components of the basic and inducible gene regulatory ma- 
chinery $^{49-51}$ resulting in modulation of C/EBP transcriptional activity. In TAD, LCR are thought to serve as flexible connection hinges or scaffolds providers for regulatory protein complex assembly. ${ }^{40} \mathrm{CR} 6$, present in all C/EBPs, contains a target for a post-translational protein modification SUMOylation involved in repressive C/EBP regulation. ${ }^{52}$ In C/EBP $\beta$ CR5 and CR7 are reported to be involved in repressive and auto-inhibitory regulation circuits, ${ }^{40}$ with no clearly revealed function for C/EBPS yet.

\subsubsection{Localization and dimerization of C/EBP $\delta$}

C/EBPS TF is widely considered to be a predominantly nuclear protein. ${ }^{36,53}$ While NF-kB, CREB or AP-1 functionally related TFs display signal-mediated translocation toward nucleus, C/EBPS immediately translocates to the nucleus after protein synthesis and does not exist in an inactive form in cytoplasm. ${ }^{36}$ As mentioned previously, NLS of C/EBPS is located in the basic DNA binding domain (DBD and FORK) (Fig.1-2). However, removal of either the basic (BA) or leucine-zipper (LEU-ZIP) domains prevents C/EBPS nuclear translocation proposing a further necessary elements located in bZIP despite DBD and FORK. ${ }^{54}$ The IPO4 importin has been reported as one of the C/EBP $\delta$ nuclear importers. ${ }^{55}$ C/EBPS TF requires dimerization for DNA binding. ${ }^{36}$ Dimerization of C/EBP $\delta$ occurs through the leucine zipper of the bZIP domain. In LEU-ZIP, 5 to 6 leucine amino acid residues at 7 residue intervals allow dimerization by hydrophobic interactions with leucine residues of the partner protein. ${ }^{36}$ Due to the high conservation in bZIP domain, C/EBPs can form homo- and hetero-dimers within the C/EBP family and with other transcription factors of bZIP superfamily. However, the LEU-ZIP domains of C/EBPs are not identical proposing the individual affinity for dimerization partners for C/EBPS. ${ }^{34}$

\subsubsection{Regulation of CEBPD gene, C/EBP $\delta$ mRNA, and C/EBP $\delta$ protein}

Based on the CEBPD's gene structure, its transcription, translation, mRNA and protein stability, C/EBPS is considered to be mainly regulated at the level of transcription initiation. ${ }^{36}$ However, C/EBP $\delta$ has been also found to be regulated at the level of DNA methylation, post-translational modifications, dimerization, and protein-protein interactions. It is also important to consider a potential existence of species-, tissue-, and cell-specific 
Introduction

differences in mechanisms of C/EBPS regulation. ${ }^{56-58}$

Up to date, $92 \mathrm{TFs}^{2 *}$ are experimentally confirmed by chromatin immunoprecipitation sequencing (ChIP-Seq) to bind human CEBPD promoter. ${ }^{59}$ The proximal CEBPD promoter region, between -300 and TSS, contains most of the characterized TF binding sites $^{36}$ (Fig.1-3A). Binding here, cAMP responsive element-binding protein (CREB), specificity protein 1 (SP1), and signal transducer and activator of transcription 3 (STAT3) belong to the most important TFs regulating C/EBP $\delta$ expression. ${ }^{36}$

Although, multiple putative CAMP responsive element (CRE) binding sites were identified in human CEBPD gene, ${ }^{60}$ only the most proximal one $(-47 /-39)$ has been reported to be functional ${ }^{36}$ (Fig.1-3A, B). Sequences of this CRE binding site are very similar in mouse and human suggesting similarities in human CEBPD and murine cebpd gene transcription regulation. ${ }^{60}$ Binding of CREB here is reported to be important for basal C/EBP $\delta$ expression in murine macrophages ${ }^{61,62}$ and CAMP-induced C/EBPS expression in mouse embryonic fibroblasts. ${ }^{60}$ It is also proposed to be involved in adipogenesis initiation. ${ }^{60}$ In neurons, in vitro data demonstrate the CREB-mediated control of neuronal C/EBP $\delta$ expression. ${ }^{63}$

Human CEBPD gene is regulated by SP1 through a proximal site $(-58 /-52)$, which is conserved in human and mouse ${ }^{64}$ (Fig.1-3A, B). This site is reported to be important for IL-6 induced C/EBPS expression in human hepatocytes ${ }^{64}$ and to be required for growth arrest induced C/EBP $\delta$ expression in human mammary epithelial cells. ${ }^{65}$ The CpG methylation of this site in human breast cancer and leukemia cell lines is related to a reduced C/EBP $\delta$ expression. ${ }^{66,67}$

In human, STAT3 transcription factor regulates CEBPD transcription through its binding to the acute phase response element (APRE) binding site (-281/-273) (Fig.1-3A, B), which is also conserved in mouse. ${ }^{65}$ This site is considered to be significant for IL-6-induced C/EBP $\delta$ expression during the acute phase response in human hepatocytes ${ }^{64,68}$ and for growth arrest induced C/EBP $\delta$ expression in mouse epithelial cells and adipocytes. ${ }^{65,69}$

\footnotetext{
2* CEBPD gene, ENCODE transcription factor targets. Harmonizome internet site. http://amp.pharm.mssm.edu/Harmonizome/gene/CEBPD. Accessed April 26, 2020.
} 


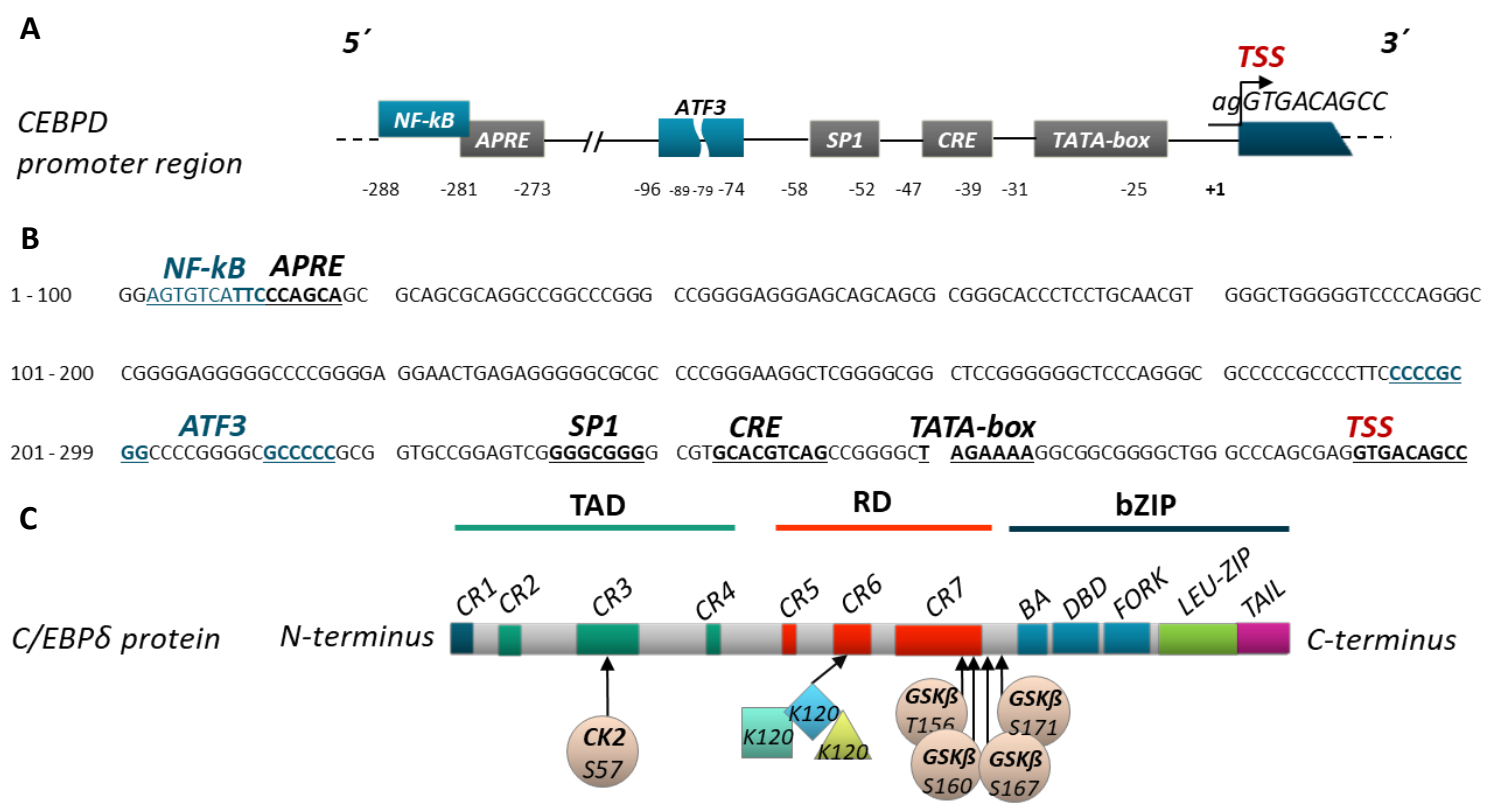

Figure 1-3: Transcriptional and post-translational regulation of C/EBPS. A, B: Identities (A, B), positions $(A)$, and sequences $(B)$ of known and proposed binding sites of regulatory TFs in the proximal CEBPD promoter (-300 bp relative to TSS). To the known and most characterized regulatory proteins belong CREB, SP1, and STAT3 TFs that bind CRE, SP1, and APRE binding sites (grey boxes, black letters), respectively. Binding sites of NF-kB and ATF3 TFs (blue boxes, blue letters) are displayed only as proposed regulatory sequences for human CEBPD, as their functionality is demonstrated only in murine macrophages. All positions refer to the reported TSS for murine cebpd gene, due to the lack of consensus regarding TSS position in human CEBPD. C: C/EBPS TF regulation by post-translational modification. Phosphorylation, the most characterized modification, is mediated by GSK $\beta$ kinase at T156, S160, S167, and by CK2 at S171 and at S57 (light orange circles). Lysine 120 represents an important target site for multiple modifications: acetylation (green square) by unknown enzyme; SUMOylation (blue rhomb) by SUMO2, and SUMO3; and ubiquitination by FBXW7 $\alpha$ and SIAH2 E3 ubiquitin ligase (yellow triangle). Number indicates the amino acid position in C/EBPS protein. S: serine; T: threonine; K: lysine.

Nuclear factor kappa-light-chain-enhancer of activated B cells (NF-kB) also belongs to the set of TFs that can influence C/EBPS expression. However, less robust evidences about NF-kB-mediated C/EBPS regulation has been reporter for human. Although, various studies demonstrate the involvement of NF-kB in C/EBPS protein expression, ${ }^{70}$ no functional NF-kB binding site in the CEBPD promoter has been clearly identified. ${ }^{36}$ In mouse $M \varphi$, a non-canonical NF-kB site is identified and reported to be essential for bacterial lipopolysaccharide- (LPS) and peptide-glycan-induced cebpd gene transcription. ${ }^{71}$ Through the alignment of the mouse cebpd and human CEBPD promoter sequences (chapter 8.1.2, Fig.8-2), the mentioned murine NF-kB binding site is located at -288/-281 position in the human sequence, partly overlapping with APRE (Fig.1-3A, B). Sequences between murine and human DNA are very similar at that point (Fig.8-2).

In mouse $M \varphi$, activating transcription factor 3 (ATF3) binds the cebpd promoter four hours after LPS treatment resulting in repression of cebpd transcription. ${ }^{71} \mathrm{~A}$ putative 
ATF3 binding site is identified in murine cebpd promoter. ${ }^{71}$ The alignment of the mouse cebpd and human CEBPD promoter sequences (Fig.8-2) revealed the corresponding ATF3 binding site position at -96/-74 in human (Fig.1-3A, B). Although, the human and murine sequences of ATF3 binding site are very similar, in human CEBPD promoter the binding site is disrupted at this position and may be non-functional (Fig.1-3B, Fig.8-2). As human CEBPD promoter contains ATF3 binding site, ${ }^{3 *}$ it must be located more distally (> $300 \mathrm{bp}$ ) relative to TSS.

DNA methylation is also suggested to represent a mechanism of C/EBPS expression regulation (Fig.1-4A). The CEBPD gene sequence displays a high frequency of CpG and encompasses a large $\mathrm{CpG}$ island that includes the whole transcribed region and up to $1 \mathrm{~kb}$ upstream of the TSS. ${ }^{36}$ Hypermethylation of proximal CEBPD promoter is suggested to be associated with reduced C/EBP $\delta$ expression in human cancers. ${ }^{72}$ However, the effect of changes in DNA methylation on C/EBPS function has not been well-characterized yet.

At the level of mRNA synthesis (Fig.1-4B) and protein translation (Fig.1-4C), single RNAbinding protein Hu antigen (HuR) is reported to bind 3'UTR of C/EBP $\delta$ mRNA leading to the increased C/EBP $\delta$ protein expression. ${ }^{36,38}$ Such stabilization of C/EBP $\delta$ mRNA by HuR is observed in growth arrested murine MCF-12A, human mammary epithelial cells, ${ }^{73}$ and human macrophages. ${ }^{74}$ In macrophages, regulatory microRNA let-7c is observed to downregulate C/EBPS mRNA by the targeting mechanism that requires the $3^{\prime} \mathrm{UTR}^{.75}$

Post-translational modifications of C/EBPS (Fig.1-4D) can affect its transcriptional activity (toward activatory or inhibitory trans-activity), DNA binding ability, protein stability, and interaction with other transcription factors and regulatory proteins. ${ }^{36}$ However, only a few post-translational modifications for C/EBP $\delta$ are described to date. The commonly reported post-translational C/EBPS modifications are phosphorylation and SUMOylation, however, acetylation and ubiquitination are also observed. ${ }^{36,76}$

Experiments with phosphatase inhibitors revealed that C/EBP $\delta$ needs to be phosphorylated to bind DNA. ${ }^{77}$ Moreover, de-phosphorylation resulted in inhibited C/EBP $\delta$ protein DNA binding and transcriptional activity. ${ }^{77} \mathrm{C} /$ EBP $\delta$ protein is phosphorylated by GSK3 $\beta$

\footnotetext{
3* CEBPD gene, ENCODE transcription factor targets. Harmonizome internet site. http://amp.pharm.mssm.edu/Harmonizome/gene/CEBPD. Accessed April 26, 2020.
} 


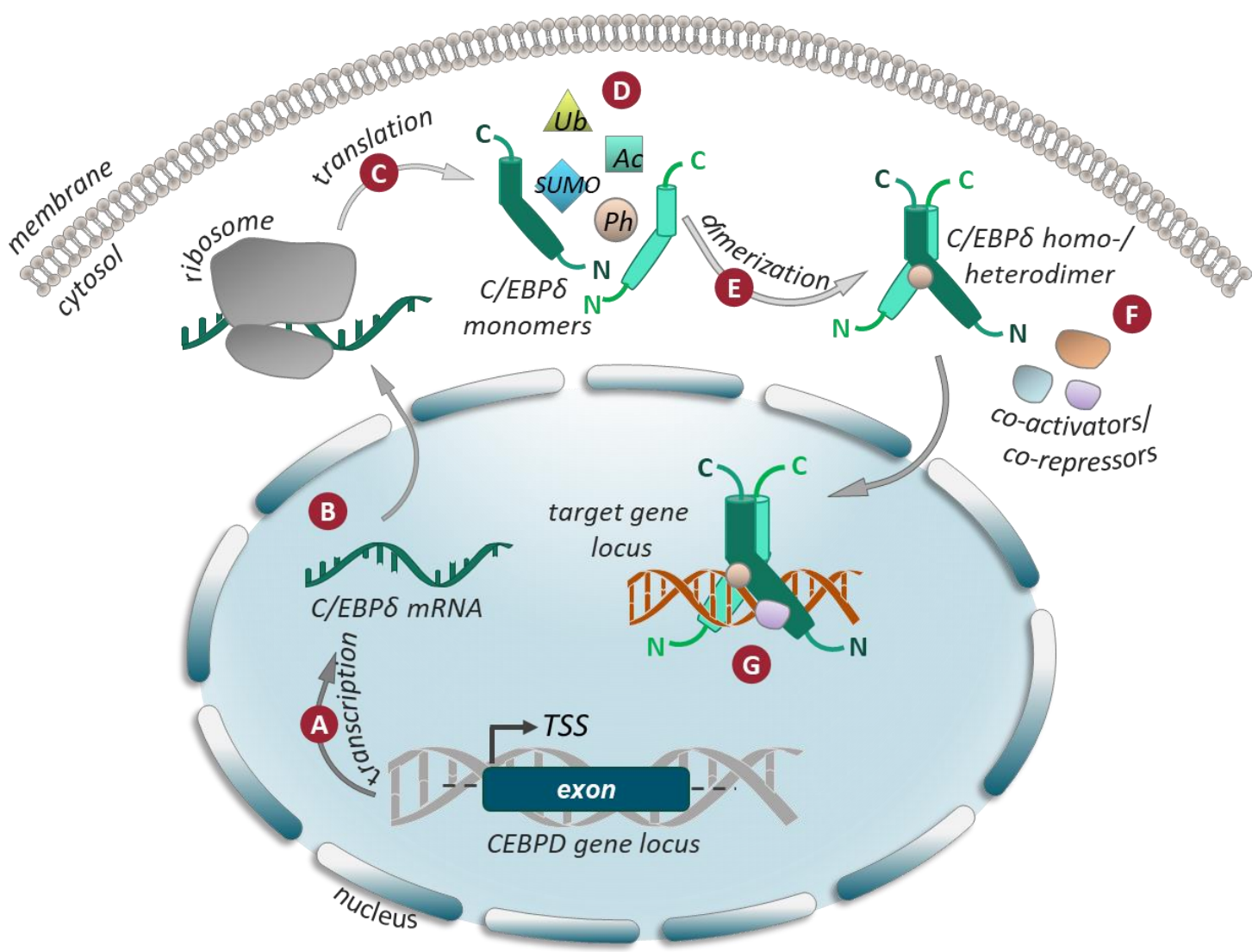

Figure 1-4: Overview of C/EBPס regulation. $A$ : Initiation of CEBPD gene transcription is the primary regulatory mechanism of C/EBP $\delta$ expression. Regulatory TFS CREB, STAT3 and SP1 are reported to bind CEBPD promoter activating CEBPD gene transcription. Methylation of CpG-rich CEBPD gene, however, results in inhibited gene expression. B: Synthetized C/EBP $\delta$ mRNA, which is highly unstable (half-time 35-40 min), can be stabilized by HuR or downregulated by microRNA let-7c. C: Generated C/EBP $\delta$ mRNA is translated into a single functional full-length $\mathrm{C} / \mathrm{EBP} \delta$ protein. D: C/EBPS protein is post-translationally phosphorylated leading to its dimerization and therefore activation. C/EBP $\delta$ can be also acetylated or SUMOylated resulting in activation or inhibition of C/EBPS target gene transcription, respectively. Ubiquitination is required for the proteasomal C/EBP $\delta$ protein degradation. E: C/EBP $\delta$ builds homo- or hetero-dimers within C/EBP family or a larger bZIP cluster. Dimerization of C/EBP $\delta$ can affect its DNA binding affinity and transactivation potential depending on the dimerization partners. F, G: Protein-protein interactions can affect C/EBP $\delta$ trans-activation potential: interaction with co-activators results in activation, while interaction with co-repressors - in inhibition of C/EBP $\delta^{\prime}$ s target genes.

kinase on Thr156 and probably Ser160 in M $\varphi$ and on Ser167 and Thr171 in astrocytes ${ }^{78}$

(Fig.1-3C). In opposition to Thr171 phosphorylation, which is constitutive, Ser167 phosphorylation is induced by IL-1 $\beta$ and is reported to be involved in C/EBP $\delta$ protein regulation during astroglial migration and astroglial-induced microglial activation. ${ }^{78}$ Further, LPS induces C/EBPS phosphorylation in IKKi/IKKe-mediated manner resulting in expression of inflammatory genes. ${ }^{79} \mathrm{C} / \mathrm{EBP} \delta$ is also phosphorylated by casein kinase 2 (CK2) on Ser57 (Fig.1-3C) leading to the enhanced C/EBP $\delta$ transcriptional activity. ${ }^{80}$

Acetylation of C/EBP $\delta$ protein by $\mathrm{p} 300 / \mathrm{CBP}^{81}$ at $\mathrm{L} 81, \mathrm{P} 82^{82}$, and $\mathrm{K} 120^{83}$ (at least in vitro) residues results in its activatory trans-potential. SUMOylation of C/EBP $\delta$ at K120 residue, 
however, eliminates its interaction with p300/CBP co-activator leading to C/EBP $\delta$ functioning as transcriptional repressor. ${ }^{83}$

An important residue in C/EBPS protein is Lys120 that is required for three different types of C/EBPS post-translational modification: acetylation, SUMOylation, and ubiquitination. ${ }^{84}$ The Lys 120 acetylation results in activatory C/EBP $\delta$ trans-activity leading to the enhanced COX2 expression, whereas the particular acetylation mechanism of C/EBPS remains unclear. ${ }^{83}$ The SUMO1, ${ }^{52}$ SUMO2, ${ }^{85}$ and SUMO3 ${ }^{85}$ proteins can be attached to Lys120 leading to an inhibitory C/EBP $\delta$ trans-activity by preventing its interaction with $\mathrm{p} 300^{83}$ and allowing recruitment of HDACs. ${ }^{86}$ Sumoylated C/EBP $\delta$ modulates HDAC4 recruitment to the COX2 promoter leading to reduced COX2 expression, whereas a non-sumoylated C/EBPS restores COX2 transcription. ${ }^{86}$ The ubiquitination of C/EBP $\delta$ at Lys120 by the ubiquitin ligase F-box and WD repeat domain containing protein 7 alpha (FBXW7 $\alpha$ ) is required for C/EBPS protein degradation, ${ }^{36}$ which is suggested to be mediated by SIAH2 ${ }^{84}$ Thus, the Lys120 poly-ubiquitination of C/EBPS by the SIAH2 E3 ubiquitin ligase leads to C/EBP $\delta$ proteasomal degradation in breast cancer cells ${ }^{84}$ and macrophages. ${ }^{36}$

At the level of protein stability, C/EBP $\delta$ is downregulated by the mentioned mechanisms involving SIAH2 E3 ubiquitin ligase ${ }^{84}$ and FBXW7 $\alpha$ poly-ubiquitination complex. ${ }^{87}$ The FBXW7 $\alpha$-mediated C/EBP $\delta$ degradation depends on prior T156 phosphorylation by GSK3 $\beta$ kinase, ${ }^{38}$ whose inhibition in turn results in C/EBPS stabilisation. Thus, in LPSactivated mouse macrophages the TLR-mediated inhibition of GSK3 $\beta / F B X W 7 \alpha$ pathway is observed to contribute to the advanced C/EBP $\delta$ protein stability. ${ }^{87}$

Both, DNA binding affinity and trans-activation potential of C/EBP $\delta$ is reported to be affected by dimerization ${ }^{36}$ (Fig.1-4E). As mentioned previously, C/EBP $\delta$ is able to form homo- and heterodimers within a C/EBP family and a bZIP class of transcription factors. ${ }^{88}$ The heterodimerization of C/EBPS with other C/EBPs doesn't affect its DNA binding affinity, ${ }^{34}$ due to the intra-familiarly conserved DBD, but can result in a changed C/EBP $\delta$ trans-activation, mediated by a variable $\mathrm{N}$-terminus of the dimerization partner. ${ }^{36}$ Heterodimerization with LIP (isoform of $\mathrm{C} / \mathrm{EBP} \beta$ ), ${ }^{89} \mathrm{C} / \mathrm{EBP} \gamma,{ }^{90}$ and $\mathrm{CHOP}^{91}$ is observed to result in an inhibitory C/EBP $\delta$ trans-activity. Within a bZIP class containing more than 100 
members including c-foc, c-jun, CREBs, and ATFs, ${ }^{92} \mathrm{C} / \mathrm{EBP} \delta$ is shown to heterodimerize with B-ATF1, B-ATF3, ATF4, and ATF5. ${ }^{88}$ The trans-activation potential and DNA binding specificity of such C/EBPS heterodimers is often different compared to its homodimers. ${ }^{56,57}$ Belonging to the stress response factors, CHOP and ATFs display well-characterized roles in hypoxia and the unfolded protein response (UPR). ${ }^{93}$ However, the specific roles of C/EBPS - CHOP/ATFs heterodimers in C/EBPS physiology function have been not specified yet. ${ }^{38}$

Protein-protein interactions of C/EBPS with other co-factors is an additional regulatory level of C/EBPS trans-activation (Fig.1-4F). Among all C/EBPs, C/EBP $\delta$ displays a unique trans-activation potential, the mediation of which also occurs through the association of C/EBPS with co-activators and co-repressors. ${ }^{36}$ As already mentioned, co-factors interact with C/EBPS via its $\mathrm{N}$-terminal RDs and TADs (Fig.1-2). The physical interaction of C/EBPS with SP94 and ZNF638 ${ }^{95}$ results in activation of C/EBP $\delta$ target genes, whereas an interaction with $\operatorname{Rad}^{96}$ or DIPA ${ }^{97}$ leads to the C/EBPS- mediated inhibition of target gene expression (Fig.1-4G). Interaction of C/EBPS with HDAC1 ${ }^{98}$ or FOXO3a ${ }^{99}$ contributes to the repression or activation of PPAR- $\gamma$ gene, respectively. C/EBPS is also reported to physically interact with Smad3 and Smad $4^{36}$ resulting in an inhibitory trans-activation function of $C / \mathrm{EBP}^{76,100}$ resulting in repression of C/EBPS target genes.

\subsubsection{Physiological function of C/EBP $\delta$}

Due to its versatile functions, C/EBP $\delta$ is considered to act as a responsive integrator of signal-dependent pathways in a cell type-specific and context-specific manner. ${ }^{38}$

C/EBPS TF is assumed to bind target gene promoters in agreement with a DNA binding model for bZIP transcription factors. ${ }^{39,101}$ Here, C/EBP $\delta$ dimer forms an inverted Y-shape structure, with each arm containing a basic region binding to one half of a C/EBP recognition sequence in the DNA major groove. ${ }^{32}$ Amino acid sequence of the DBD in C/EBP $\delta$ dictates its DNA binding specificity. ${ }^{102}$ The palindromic symmetrical repeat RTTGC GYAAY, with purines $(R)$ and pyrimidines $(Y)$ is determined as an optimal C/EBP binding site, ${ }^{103}$ whereas more complex consensus patterns are also described. ${ }^{104}$

Initially, C/EBPS is reported to serve as a marker for adipocyte ${ }^{105}$ and myeloid differenti- 
ation ${ }^{106}$ or inflammatory activation. ${ }^{38}$ To date, C/EBP $\delta$ is also known for transcriptionally modulating of cellular differentiation, ${ }^{106,107}$ macrophage polarization, ${ }^{75}$ proliferation, ${ }^{108,109}$ growth arrest, ${ }^{106}$ cell survival and cell death, ${ }^{110}$ metabolism, ${ }^{111}$ learning and memory formation, ${ }^{112}$ and immune responses (chapter 1.3.2.1). In most cells, basal C/EBP $\delta$ expression level is typically low, which can be rapidly induced in response to various stimuli including glucocorticoids, ${ }^{113}$ hormons, ${ }^{98,114}$ growth factors, ${ }^{115}$ and inflammatory factors (chapter 1.3.2.1). Regulation of gene transcription in the context of inflammation belongs to the key functions of C/EBP $\delta$.

\subsubsection{Role of C/EBP $\delta$ in the context of inflammation and related disorders}

\subsubsection{C/EBPS in inflammatory process}

Inflammation is a protective response of organism to pathogens and cell or tissue damage. It is mediated by multiple interactions of immune cells and regulated by various molecular mediators as a part of the corresponding signalling pathways. The complex inflammatory response is suggested to be mainly regulated at the level of gene transcription. ${ }^{116-118}$ C/EBPS TF is known to be predominantly involved in transcriptional control of inflammation, acting as a key regulator of inflammatory responses. ${ }^{36,119}$ Various pro-inflammatory stimuli including LPS, ${ }^{58,71,120-123}$ IFN-g, ${ }^{58,123}$ IFN- $\alpha$, ${ }^{58}$ TNF- $\alpha,{ }^{58,122,124}$ IL-6, ${ }^{64,125}$ IL-1, ${ }^{58,123}$ IL-1 $\beta,{ }^{32,78,126}$ but also glucocorticoids ${ }^{113,127}$ and PGE2 ${ }^{74,128}$ activate C/EBPS (Fig.1-5). During inflammation, signalling pathways involving PI3 kinase, ${ }^{129}$ p38 MAPK, ${ }^{78,115} \mathrm{JAK}^{125} \mathrm{JNK}^{130}$ and PKA ${ }^{128}$ mediate C/EBPS activation. Inflammation-induced C/EBPS TF regulates or co-regulates a number of inflammatory genes, including TNF- $\alpha{ }^{131,132}$ IL-1, ${ }^{131}$ IL-1 $\beta,{ }^{132}$ IL- $6,{ }^{131,132}$ CXCL1, ${ }^{132}$ IL-17A, ${ }^{132}$ IL-8, ${ }^{131}$ IL-12, ${ }^{131}$ COX2, ${ }^{83,131}$ iNOS, ${ }^{131}$ chemokines, monocyte chemoattractant protein 1 (MCP1) and anti-inflammatory IL-10 $74,78,130,133$ (Fig.1-5). Additionally, multiple cell types including $M \varphi{ }^{71,75,79,87,134}$ tumor associated macrophages (TAMs), ${ }^{74}$ hepatocytes, ${ }^{121,135}$ and astroglial cells ${ }^{122,123}$ express C/EBP $\delta$ in an inflammation-mediated manner.

The pro-inflammatory regulatory activity of C/EBPS is mostly studied in macrophages $(\mathrm{M} \varphi)$, cells of innate immune system that normally acts as the primary defence line against pathogens. Pro-inflammatory activated $\mathrm{M} \varphi$ are suggested to contribute complex pathogenesis of various disorders including atherosclerosis, ${ }^{136}$ cancer, ${ }^{137}$ and rheu- 
Introduction

matoid arthritis. ${ }^{138,139}$

Responses of the innate immune system are largely triggered by toll-like receptors (TLRs), pattern recognition receptors that mediate immune responses to infection. ${ }^{140}$ Expressed on $M \varphi$, TLRs recognise microbial components ${ }^{141,142}$ and activate corresponding signalling pathways resulting in the induction of critical pro-inflammatory genes $^{143,144}$ including C/EBPS. Inflammatory responses in $M \varphi$ are also suggested to be mainly driven at the level of transcription. ${ }^{116-118}$ TLR4 agonist LPS, ${ }^{145}$ a main component of gram-negative bacteria surface, induces a battery of genes in $M \varphi^{146}$ resulting in $M \varphi$ activation. ${ }^{71}$ In LPS-activated $\mathrm{M} \varphi$, three classes of transcriptional factors regulate transcription initiation of response genes. ${ }^{118}$ Class I transcription factors (e.g. NF-kB) are activated post-transitionally, often at the stage of nuclear translocation, up to two hours after LPS exposure. ${ }^{118}$ These transcription factors control the induction of the primary response genes. Class II transcription factors (e.g. C/EBPS, ATF3), which are induced during primary response four to six hours after LPS binding, control the induction of the secondary response genes. ${ }^{118}$ Induced during secondary response, class III transcription factors (e.g. C/EBP $\beta$, PU.1) are not directly targeted by pro-inflammatory signals but are responsible for specifying of $M \varphi$-specific gene expression pattern. ${ }^{118}$

LPS-induced activation of $\mathrm{M} \varphi$ results in production and secretion of pro-inflammatory cytokines and chemokines, enhanced co-stimulatory receptors expression required for T-cell activation, enhanced production of metabolites, and changes in phagocytosis-related systems. ${ }^{143,144,147,148}$ This complex LPS signalling network in M $\varphi$ includes C/EBPסdependent regulatory mechanisms.

Murine $M \varphi$ display an important regulatory loop that involves C/EBPS, NF-kB, and ATF3 LPS-responsive genes. ${ }^{71}$ Here, TLR4-activated NF-kB directly activates and is necessary for C/EBPS expression, which is attenuated by TLR4-activated ATF3 ${ }^{71}$ (Fig.1-5). Moreover, LPS-activated C/EBPS binds its own promoter in an auto-regulatory manner resulting in activation of C/EBP $\delta$ transcription. ${ }^{71}$ Such complex interactions in NF-kB-ATF3C/EBPS regulatory network result in a fine-tuned gene regulation of pro-inflammatory IL-6. Gene expression of IL- 6 is fully activated by the combined action of both NF-kB and NF-kB-dependent C/EBPS, whereas the single NF-kB binding elicits only a weak IL-6 expression. ${ }^{71}$ Facilitating the sustained expression of the inflammatory genes, C/EBP $\delta$ acts 


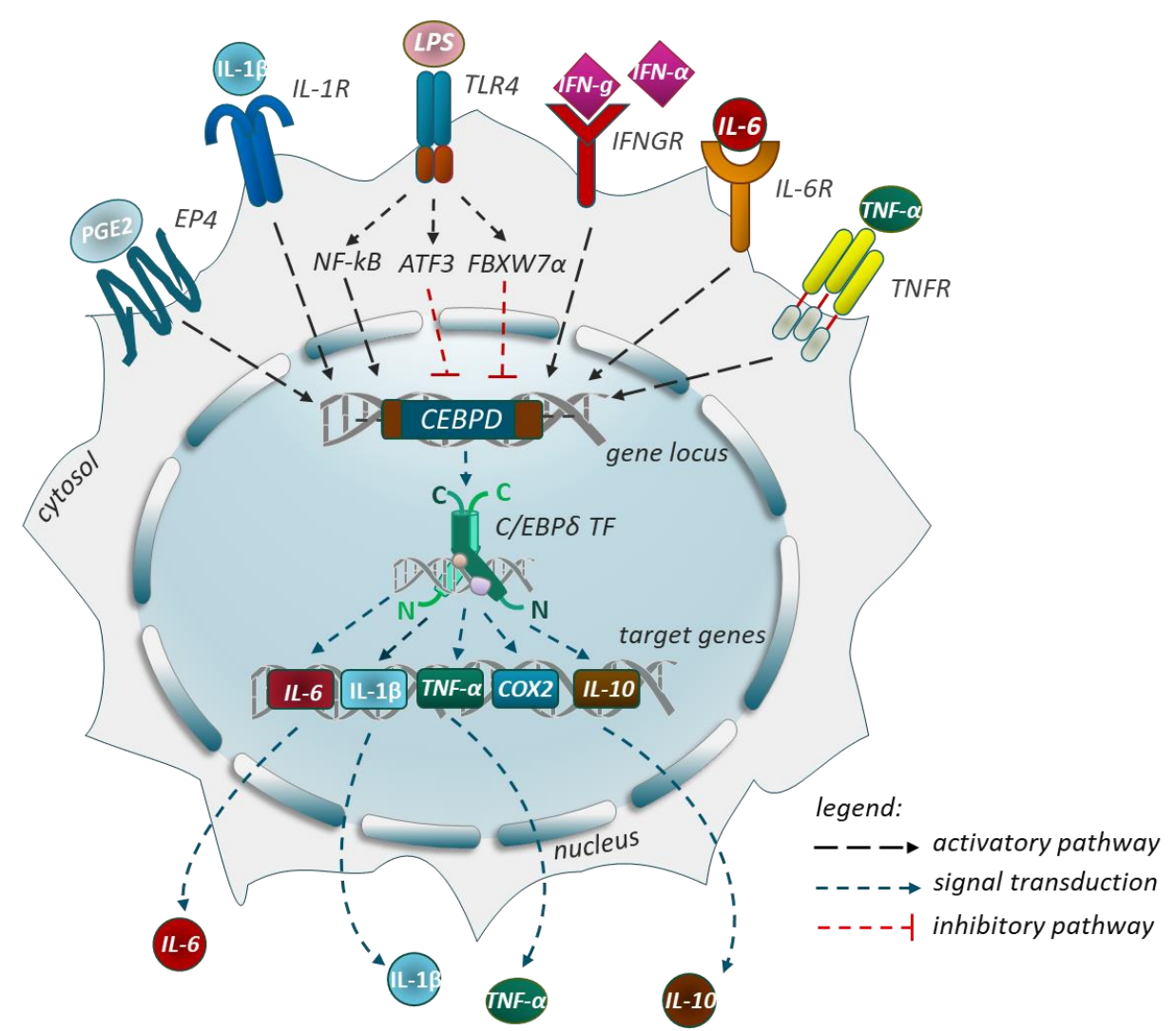

Figure 1-5: Simplified overview of CEBPD-mediated gene transcription in M $\boldsymbol{\varphi}$. CEBPD gene expression is activated by various stimuli including LPS, IFN-g, IFN- $\alpha$, TNF- $\alpha$, IL-6, and other. The LPS-mediated CEBPD expression is induced by NF-kB and attenuated by ATF3 and FBXW7 $\alpha$ through multiple regulatory mechanisms and loops. In LPS-activated $M \varphi$, CEBPD acts as a context-specific signal integrator that regulate expression of pro- (e.g. IL-6, IL-1ß, etc.) and anti-inflammatory (e.g. IL-10) target genes affecting the final $\mathrm{M} \varphi$ phenotype. For details refer to the main text. EP4: E-type prostanoid receptor 4; IL-1R: interleukin 1 receptor; TLR4: toll-like receptor 4; IFNGR: interferon gamma receptor; IL-6R: interleukin 6 receptor; TNFR: tumor necrosis factor receptor.

as an amplifier of the transient transcriptional activity of NF-kB. ${ }^{71,131}$ Such C/EBP $\delta$-mediated recognition of transient and persistent TLR4 signals enables the native immune system to mediate correct immune response. ${ }^{71}$ However, NF-kB-ATF3-C/EBP $\delta$ regulatory loop is rather cell-specific, as it is absent in kidney fibroblasts, where C/EBP $\delta$ expression is induced by c-Jun and maintained by an IRAK-1-dependent mechanism. ${ }^{149}$

In activated $M \varphi, C / E B P \delta$ is observed to amplify LPS signalling, to support expression of LPS-responsive genes, and to be essential for clearance of persistent bacterial infection. ${ }^{71}$ Identified by the "chip-on-chip" analysis in murine M $\varphi$, TLR4-stimulated C/EBP $\delta$ is responsible for direct activation of 63 LPS-induced genes, including Serpinb2, Cp, Saa3, Hp, Camp, C3, Tnfaip6, Ccl3, Cxcl2, and F10. ${ }^{71}$ In response to persistent LPS treatment, the transcription of these C/EBP $\delta$ target genes is significantly diminished in C/EBP $\delta$-deficient murine $M \varphi .^{71}$ 
Analysis of C/EBP $\delta$-deficiency further reveals its role in the context of inflammation. C/EBPS-null M $\varphi$ display reduced induction of IL- 6 and TNF- $\alpha$ in response to several TLR ligands. ${ }^{34,150,151}$ In peritoneal $\mathrm{M} \varphi, \mathrm{C} / \mathrm{EBP} \delta$-deficiency is observed to affect the expression of several TLR genes, ${ }^{87}$ not only of reported TLR4. Decreased plasma levels of TNF- $\alpha$ and IL-6 are reported to contribute to a reduction of endotoxin-induced systemic inflammation in C/EBPS-null mice. ${ }^{152}$ Further, systemic LPS-induced gene expression of iNOS, TNF- $\alpha$, IL-1 $\beta$, and IL- 6 is observed to be attenuated in glia cells of C/EBP $\delta$ knock out mice. ${ }^{153}$ Further, C/EBPS-deficiency is reported to protect against LPS-induced lung injury ${ }^{151}$ and (at least partly) from sepsis. ${ }^{152}$

However, C/EBPS TF is also observed to display an anti-inflammatory activity. ${ }^{38,119}$ C/EBPS mediates LPS- or PGE2-induced activation of anti-inflammatory IL-10 in mouse $\mathrm{M} \varphi,{ }^{74,130}$ facilitates anti-apoptosis, and attenuates IL-1ß and IFN-g-induced production of chemokines by promotion of IRF-1 expression in rat pancreatic ß-cells. ${ }^{154}$ The activated C/EBPS TF may prevent inflammatory responses in human pericytes, ${ }^{155}$ protect against radiation-induced sepsis suppressing inflammation. ${ }^{156}$

\subsubsection{C/EBPS in the context of inflammatory disorders}

Activation of C/EBPS gene transcription is observed in acute inflammatory ${ }^{119}$ and chronic inflammatory diseases including atherosclerosis, ${ }^{157,158}$ type 2 diabetes, ${ }^{159}$ Parkinson's disease, ${ }^{160,161}$ Alzheimer's disease, ${ }^{162,163}$ and rheumatoid arthritis. ${ }^{132,164}$ C/EBP $\delta$ is also involved in regulation of neuro-inflammation ${ }^{153,163}$ and cancer pathology. ${ }^{38,74,108}$ This study focuses on implementation of C/EBP $\delta$ in the pathology of rheumatoid arthritis.

\subsubsection{Rheumatoid arthritis and the macrophage C/EBPS}

Rheumatoid arthritis (RA) is a chronic inflammatory disease characterised by inflammation-driven pannus formation that affects peripheral joints resulting in cartilage and bone destruction. ${ }^{165}$ Complex pathophysiology of RA is further underlined by invasion of inflammatory cells, proliferation and migration of synoviocytes, angiogenesis, and activity of bone-destructive chondrocytes and osteoclasts. ${ }^{165,166}$ 
Synovial membrane of a normal joint capsule displays rather low cell type variety containing scarce blood vessels and $М \varphi$-encompassing fibroblast tissue in the inner joint lining $^{167}$ (Fig.1-6A). In contrary, the synovial membrane of RA joint becomes hypertrophic ${ }^{168}$ and promotes the formation of a pannus - an invasive and destructive front built of fibroblast-like synoviocytes. ${ }^{165}$ In pannus, chronic inflammatory condition is generated by the activity of infiltrated immune cells including $M \varphi$, plasma and mast cells, dendritic cells, neutrophils, and T- and B-lymphocytes ${ }^{165,166}$ (Fig.1-6A).

Inflammatory-activated $\mathrm{M} \varphi$ are suggested to represent central cells in RA pathology, ${ }^{169-}$ 172 due to their prominent number in the synovial membrane ${ }^{173,174}$ and cartilage-pannus junction. ${ }^{175}$ During inflammation, $\mathrm{M} \varphi$ undergo polarization toward a pro-inflammatory M1 state, induced by various stimuli including LPS and cytokine interferon gamma (IFN-g). ${ }^{172}$ Whereas IFN-g elicits cellular responses by binding of cytokine receptors, cell activation markers, and cell adhesion molecules, ${ }^{176,177}$ LPS activates various inflammation-linked signaling pathways acting via TLRs. ${ }^{172}$ In RA, M1-polarized pro-inflammatory $M \varphi^{167,178,179}$ act as local and systemic disease amplifiers by production and secretion of inflammatory cytokines including TNF, IL-1ß, IL-6, IL-12, IL-23 and chemokines such as CCL5, CCL8, CXCL12, CXCL4. ${ }^{167,169,171,172}$ Via their action in autocrine and paracrine manner, $M \varphi$ activate fibroblasts, ${ }^{180-182}$ osteoclasts, ${ }^{172}$ chondrocytes, ${ }^{172}$ leucocytes, and T-cells resulting a in cartilage and bone destruction, ${ }^{183,184}$ sustained inflammatory condition and pain. ${ }^{185}$

TLR2 and TLR4 receptors, ${ }^{186,187}$ which can be also activated by intrinsic non-infectious agents, are proposed to contribute to RA pathology. ${ }^{188,189}$ In RA joint, TLR2 and TLR4 are greatly expressed by synovial lining and sub-lining $M \varphi$ as well as by fibroblasts. ${ }^{190,191}$ TLR signaling activates MyD88 pathway, important for transcriptional activation of inflammation-related genes ${ }^{192}$ including NF-kB and CEBPD. ${ }^{71}$

Cytokine IFN-g belongs to intrinsic inflammatory RA mediators, which levels significantly correlate with disease severity. ${ }^{193}$ In RA joint, infiltrating immune cells like neutrophils ${ }^{172}$ and T-cells ${ }^{194,195}$ secrete IFN-g driving M1-polarization of $\mathrm{M} \varphi$. In $\mathrm{M} \varphi$, the IFN-g- and LPSresponsive C/EBPS TF controls expression of a battery of target genes, ${ }^{71}$ the summary action of which triggers inflammation and loss of function in RA joint. ${ }^{132,164}$ 
A

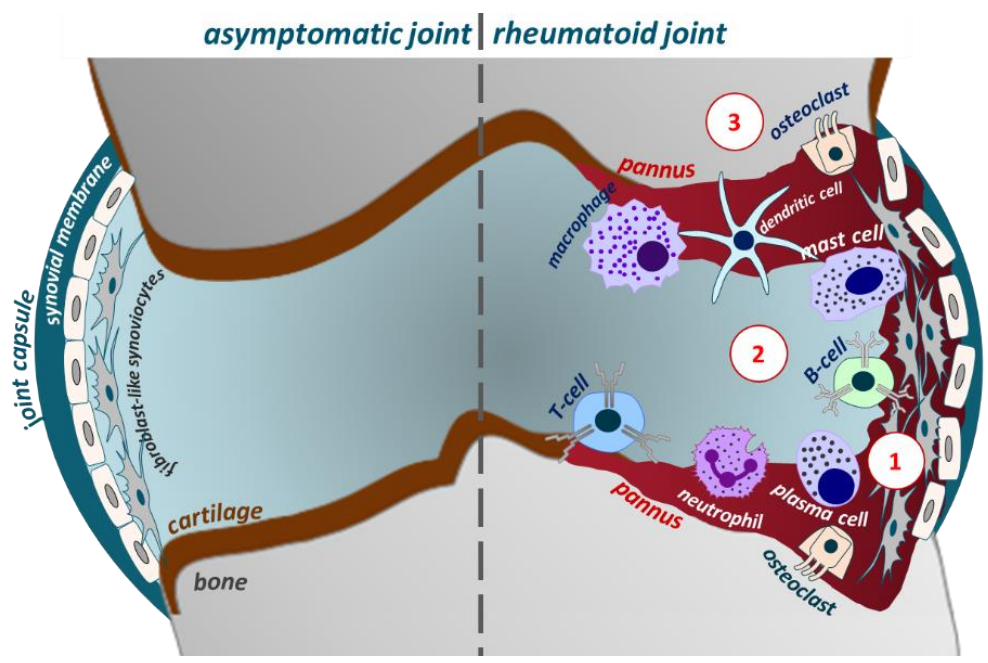

B human rheumatoid joint

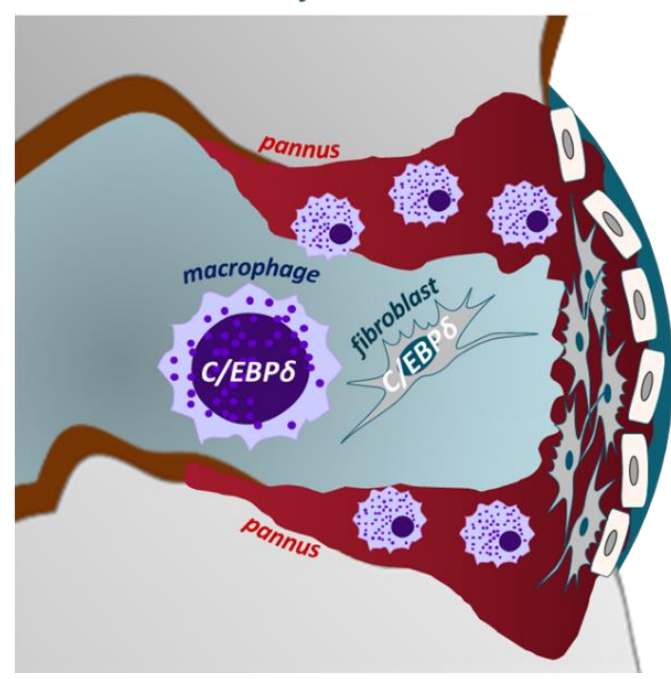

C rheumatoid joint of CIA mice

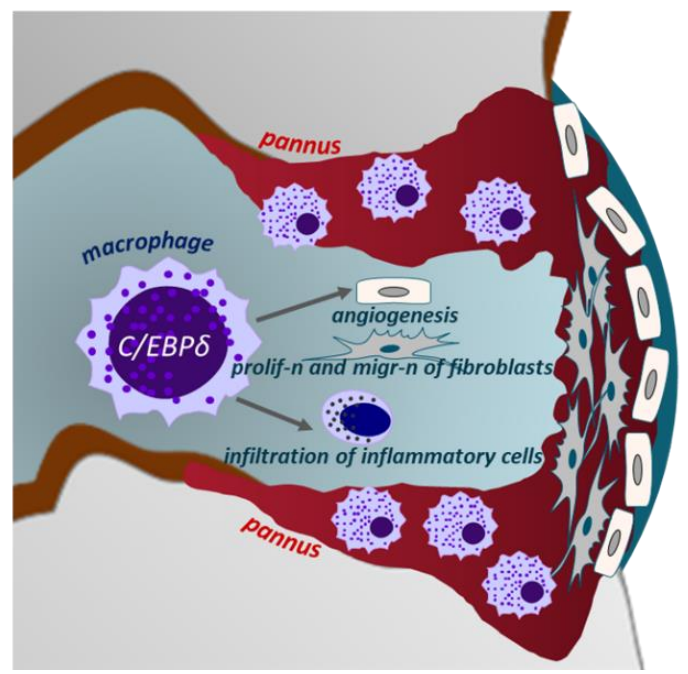

Figure 1-6: Schematic display of general and C/EBPS-mediated pathophysiology of RA. A: In a normal joint fibroblast-like synoviocytes build a tiny lining on synovial membrane, while in a rheumatoid joint these hyperplastic, proliferative cells are greatly increased in mass and form an invasive pannus front (1). Various immune cells including $M \varphi$, dendritic cells, mast cells, plasma cells, neutrophils, T- and B-cells infiltrate the pannus causing chronic inflammation. Generated inflammatory milieu is further driven by secreted cytokines and chemokines (2). As a result, inflammation-driven osteoclasts cause bone destruction contributing to the loss of joint function (3). B: In synovial tissue biopsies of RA patients, C/EBP $\delta$ TF displays an elevated DNA binding activity. Inflammation-activated C/EBPS TF predominantly localizes in the nucleus of pannus lining cells including CD4+ macrophage-like and CD4- fibroblast-like cells. C: In CIA mice, enhanced expression of macrophage C/EBP $\delta$ TF promotes angiogenesis, infiltration of inflammatory cells, as well as proliferation and migration of fibroblasts, factors that were attenuated in C/EBP $\delta$-deficient CIA mice.

The involvement of the macrophage C/EBP $\delta$ in pathophysiology of RA is shown in both, murine model of RA ${ }^{132}$ and biopsies of RA patients. ${ }^{164}$ Elevated C/EBPS DNA binding activity in RA patients' synovial tissue positively correlates with both serum C-reactive protein and synovial IL-6 mRNA levels. ${ }^{164}$ An immune-histochemical analysis reveals localization of activated C/EBPS protein predominantly in the nuclei of rheumatoid synovial lining cells including macrophage-like and fibroblast-like cells. ${ }^{164}$ Elevated C/EBPS is therefore proposed to contribute to the pathology of synovitis in RA through the activa- 
tion of gene expression in both, cytokine-producing $\mathrm{M} \varphi$ and cytokine responding fibroblast cells ${ }^{164}$ (Fig.1-6B).

As introduced previously, human CEBPD and murine cebpd gene promoter regions and corresponding proteins are highly conserved enabling function and regulation study of C/EBPS TF in mice. ${ }^{36} \mathrm{~A}$ collagen-induced arthritis (CIA) mice is a well-established murine model of RA, which displays similarities in RA symptoms such as synovitis, bone erosion, and pannus formation. ${ }^{132}$ Inflammatory-activated C/EBPS TF is proposed to contribute to disease development in CIA mice (Fig.1-6C), as C/EBPS-deficient CIA mice displays improved joint integrity, minor infiltration of inflammatory cells to the pannus, decreased pannus growth, and reduced formation of micro vessels. ${ }^{132}$

C/EBPS TF also affects mRNA expression and cytokine secretion in human THP-1 cells. ${ }^{132}$ THP-1 is a human monocyte-like cell line, which is generated by a spontaneous immortalization of monocyte cells from peripheral blood of acute monocytic leukemia (AML-M5) patient, ${ }^{196}$ widely used to study physiological and pathophysiological aspects of inflammation. ${ }^{197}$ Expression levels of over 400 genes (>1.5-fold) are significantly different in TNF- $\alpha$-treated C/EBPS-deficient THP-1 cells, as revealed by mRNA microarray analysis. ${ }^{132}$ Inflammatory-activated C/EBPS directly binds promoter regions of genes that contribute to angiogenesis, ${ }^{132}$ attract leucocytes to the synovium, ${ }^{198,199}$ and promote migration and proliferation of fibroblast-like synoviocytes in a paracrine manner, detected by ChIP analysis in THP-1 cells. ${ }^{132}$ Elevated C/EBPS levels also result in upregulated secretion of 25 proteins, as detected by a cytokine array analysis of the conditioning medium of CEBPD-overexpressing THP-1 cells. ${ }^{132}$ To these proteins belong important pro-inflammatory factors identified in RA joints: IFN-g, IL-1B, IL-6, IL-8, IL-17, IL-23A, CCL20, CXCL1, CXCR2, and TGF-ß. ${ }^{132}$

Attempts to reduce inflammation by non-steroidal anti-inflammatory drugs (NSAIDs), glucocorticoids, and disease modifying anti-rheumatic drugs (DMARDs) are the standard approach in current RA therapy. ${ }^{202,203}$ Anti-inflammatory compounds rosmanol ${ }^{200}$ and inotinol $^{201}$ inhibit TNF- $\alpha$-activated CEBPD gene expression resulting in attenuated migration and proliferation of fibroblasts and reduced neo-angiogenic tube formation by HUVECs. ${ }^{132}$ However, activation of macrophage CEBPD may also result in an anti-inflam- 
matory $M \varphi$ phenotype ( $c f$. chapter 1.3.2.1). Thus, CEBPD-modulating compounds may be potential candidates for therapy of inflammatory disorders including RA.

\subsection{Aims of the study}

Identification of anti-inflammatory compounds is a part of the ongoing development of treatment strategies of chronic inflammatory disorders like RA. Phenotypic screening is a target-agnostic drug discovery strategy that monitors screening compound-mediated changes in a system-based manner. Phenotypic screening aims to relate systemic stimulus response to a quantifiable change of phenotype such as gene expression. Gene reporter assays represent a specific category of phenotypic screening, relying on the relationship between cellular phenotype and cellular networks resulting in a measurable gene reporter activity.

Inflammatory responses in $M \varphi$ are proposed to be regulated mainly at the gene transcription level. C/EBPS transcription factor, being regulated predominantly on level of gene transcription initiation, is a primary response gene triggering downstream gene expression cascade regulating inflammatory response and defining a specific $\mathrm{M} \varphi$ phenotype. Elevated C/EBPS TF activity contributes to RA pathology, as observed in mice and human. However, activation of C/EBPS TF may result in both anti- and pro-inflammatory phenotype, functioning as a context-specific signal integrator in $M \varphi$. Therefore, both pharmacological inhibition and activation of macrophage CEBPD may display the sought after anti-inflammatory effect.

The first aim of this study was to develop a flexible, reliable, and sensitive phenotypic screening assay in M1-polarized THP-1 M $\varphi$ that mimic cellular context of inflammatory RA. Generation of the SEAP-containing gene reporter cassette, central to the screening assay flexibility, was a priority during screening assay development.

The second aim of this study was, subsequent to development of the final screening protocol, to conduct a high-throughput screening (HTS) of LOPAC ${ }^{\circledR 1280}$ and ENZO ${ }^{\circledR 774}$ libraries for CEBPD-modulating compounds with the final goal to characterize the resulting hit compounds according to their anti-inflammatory activity via gene expression regulation of CEBPD and related pro-inflammatory genes. 


\section{Materials and Methods}

\subsection{Materials, kits and devices}

The used consumables (Table 2.1), compounds, chemicals, reagents (Table 2.2), commercial kits (Table 2.3) as well as instruments, devices, and corresponding software (Table 2.4) are listed below. Instruments and devices used during a HTS on Fraunhofer IME ScreeningPort in Hamburg are noticed in Table 2.4.

Table 2.1: List of used consumables.

\begin{tabular}{|c|c|}
\hline product & supplier, location \\
\hline aerosol Barrier Pipet Tips, 0.1-1 mL, 20-200 $\mu \mathrm{L}, 2-20 \mu \mathrm{L}, 1-10 \mathrm{uL}$ & Fischerbrand $^{T M}$, Thermo Fisher Scientific, \\
\hline (Fraunhofer IME ScreeningPort) & Oberhausen, DE \\
\hline bacteria culture tube & Greiner Bio-One, Kremsmünster, AUT \\
\hline cell culture dish, $10 \mathrm{~cm}$ & Greiner Bio-One, , Kremsmünster, AUT \\
\hline cell culture flasks, $\mathrm{T} 175, \mathrm{~T} 75, \mathrm{~T} 25$ & Greiner Bio-One, , Kremsmünster, AUT \\
\hline cell culture plates, 6-, 24-, 96-, 384-well & Greiner Bio-One, , Kremsmünster, AUT \\
\hline cell scraper & Sarstedt, Nümbrecht, DE \\
\hline chambers, agarose gel elecrophoresis, 40-1214, 40-0708, 40- & Peqlab Biotechnologies, Erlangen, DE \\
\hline \multicolumn{2}{|l|}{1214} \\
\hline chambers, PAGE & Bio-Rad, Hercules, CA, USA \\
\hline Clip-tips, up to: $300 \mu \mathrm{L}, 100 \mu \mathrm{L}, 50 \mu \mathrm{L}, 10 \mu \mathrm{L}$ & Thermo Fisher Scientific, Oberhausen, DE \\
\hline combitips, up to: $10 \mathrm{~mL}, 5 \mathrm{~mL}, 1 \mathrm{~mL}, 0.5 \mathrm{~mL}, 0.2 \mathrm{~mL}, 0.1 \mathrm{~mL}$ & Eppendorf, Hamburg, DE \\
\hline Corning ${ }^{\circledR}$ CoolCell ${ }^{\circledR}$ LX, Cell Freezing Container & Corning, NY, USA \\
\hline cryo tubes, $2 \mathrm{~mL}$ & Greiner Bio-One, Kremsmünster, AUT \\
\hline cuvettes, clear & Carl Roth, Karlsruhe, DE \\
\hline Echo plates, Echo Qualified 384-well, low dead volume & Labcyte, San Jose, CA, USA \\
\hline electroporation cuvettes, Puser ${ }^{\otimes} /$ MicroPulser $^{\mathrm{TM}}, 0.1 \mathrm{~cm}$ gap & Bio-Rad, Hercules, CA, USA \\
\hline falcons, $15 \mathrm{~mL}, 50 \mathrm{~mL}$ & Greiner Bio-One, Kremsmünster, AUT \\
\hline filter, Filtropur $\mathrm{S} 0.2,0.2 \mu \mathrm{m}$ & Sarstedt, Nümbrecht, DE \\
\hline Finnpette $^{\circledR}$, multi-channel pipettes, 16-channel, manual, 5-50 & Thermo Scientific, Waltham, MA, USA \\
\hline \multicolumn{2}{|l|}{$\mu \mathrm{L}, 1-10 \mu \mathrm{L}$ (Fraunhofer IME ScreeningPort) } \\
\hline $\begin{array}{l}\text { Finnpette }^{\circledR} \text {, multi-step, multi-channel pipette, electric, 16- } \\
\text { channel, 5-50 } \mu \mathrm{L} \text { (Fraunhofer IME ScreeningPort) }\end{array}$ & Thermo Scientific, Waltham, MA, USA \\
\hline Gene Pulser ${ }^{\circledR}$ electroporation cuvette, $1 \mathrm{~mL}$ & Bio-Rad, Hercules, CA, USA \\
\hline glass coverslips, $22 \mathrm{~mm} \times 22 \mathrm{~mm}$ & Thermo Scientific, Waltham, MA, USA \\
\hline glass slides, Superfrost ${ }^{\circledR}$ Plus & Thermo Scientific, Waltham, MA, USA \\
\hline glassware & Schott, SIMAX, Duran Hirschmann \\
\hline hemocytometer, $0.1 \mathrm{~mm} \times 0.0025 \mathrm{~mm}^{2}$ & Marienfeld, Lauda-Königshofen, DE \\
\hline inoculation loop & Greiner Bio-One, Kremsmünster, AUT \\
\hline
\end{tabular}


Table 2.1: Continuance.

\begin{tabular}{|c|c|}
\hline product & supplier, location \\
\hline Mini-PROTEAN ${ }^{\circledR}$ TGX Stain-Free ${ }^{\mathrm{TM}}$ Precast gel, 8-16\% & Bio-Rad, Hercules, CA, USA \\
\hline Mr. Frosty ${ }^{\mathrm{TM}}$ Freezing Container & Thermo Fisher Scientific, Oberhausen, DE \\
\hline multi-channel pipettes, manual, 8-, 12-channel, 30-300 $\mu \mathrm{L}, 10-$ & Thermo Scientific, Waltham, MA, USA \\
\hline \multicolumn{2}{|l|}{$100 \mu \mathrm{L}, 5-50 \mu \mathrm{L}, 1-10 \mu \mathrm{L}$} \\
\hline multi-step, one-channel pipette, electric, Repeater E3 & Eppendorf, Hamburg, DE \\
\hline multi-well plate seal film & Carl Roth, Karlsruhe, DE \\
\hline multi-well plates, 96-, 384-well formats & Greiner Bio-One, Kremsmünster, AUT \\
\hline one-channel pipette tips, up to: $1 \mathrm{~mL}, 0.2 \mathrm{~mL}, 10 \mu \mathrm{L}$ & Starlab, Hamburg, DE \\
\hline one-channel pipettes, manual, 0.1-1 mL, 10-200 $\mu \mathrm{L}, 1-10 \mu \mathrm{L}, 0.1-$ & Eppendorf, Hamburg, DE \\
\hline \multicolumn{2}{|l|}{$2 \mu \mathrm{L}$} \\
\hline Parafilm $\mathrm{M}^{\circledR}$ & Pechine Plastic \\
\hline pasteur pipettes, $230 \mathrm{~mm}$ & Carl Roth, Karlsruhe, DE \\
\hline PCR foil seal (Fraunhofer IME ScreeningPort) & 4-titude ${ }^{\circledast}$, UK \\
\hline PCR tubes, $0.2 \mathrm{~mL}$, 8-strips & Biozym, Hessisch Oldendorf, DE \\
\hline petri dish & Greiner Bio-One, Kremsmünster, AUT \\
\hline \multirow[t]{2}{*}{ PipetBoy 2} & Integra ${ }^{\mathrm{TM}}$, Thermo Fisher Scientific, Ober- \\
\hline & hausen, DE \\
\hline PVDF membrane, $0.45 \mu \mathrm{m}$ & Millipore, Burlington, MA, USA \\
\hline qPCR multi-well plates, 384-well format & Thermo Fisher Scientific, Oberhausen, DE \\
\hline qPCR multi-well plates, 96-well format & Bio-Rad, Hercules, CA, USA \\
\hline qPCR plate cover foil & Greiner Bio-One, Kremsmünster, AUT \\
\hline qPCR plate seal film & Bio-Rad, Hercules, CA, USA \\
\hline qPCR strips for plate & Bio-Rad, Hercules, CA, USA \\
\hline reaction tubes, $2 \mathrm{~mL}, 1.5 \mathrm{~mL}, 0.5 \mathrm{~mL}$ & Eppendorf, Hamburg, DE \\
\hline scalpel & Heinz Herenz, Hamburg, DE \\
\hline Scepter Sensors, 60 m (Fraunhofer IME ScreeningPort) & Millipore, Burlington, MA, USA \\
\hline serological pipettes, $5-25 \mathrm{~mL}, 2-10 \mathrm{~mL}, 0.5-5 \mathrm{~mL}, 0.3-2 \mathrm{~mL}$ & Eppendorf, Hamburg, DE \\
\hline syringes, $10 \mathrm{~mL}, 5 \mathrm{~mL}, 1 \mathrm{~mL}$ & Inject $^{\circledR}, \mathrm{DE}$ \\
\hline tips for Finnpipette ${ }^{\circledR}, 16$-channel, $1-10 \mu \mathrm{L}$ & Thermo Scientific, Waltham, MA, USA \\
\hline tips for Finnpipette ${ }^{\circledR}, 16$-channel, 5-50 $\mu \mathrm{L}$ & Axygen $^{\circledR}$, Corning, NY, USA \\
\hline tips, Janus MDT P30 (Fraunhofer IME ScreeningPort) & Axygen $^{\circledR}$, Corning, NY, USA \\
\hline $\begin{array}{l}\text { Transferpipette }{ }^{\circledR} \text {, multi-step, multi-channel pipette, 8- } \\
\text { channel, electric, } 10-200 \mu \mathrm{L} \text { (Fraunhofer IME ScreeningPort) }\end{array}$ & Brand, Wertheim, DE \\
\hline water treatment plant, Milli-Q-Water-System & Millipore, Burlington, MA, USA \\
\hline whatman & Bio-Rad, Hercules, CA, USA \\
\hline 2,2',2",2"'"-(ethane-1,2-diyldinitrilo)tetraacetic acid, & Sigma Aldrich, St. Louis, MO, USA \\
\hline EDTA & \\
\hline
\end{tabular}


Table 2.2: List of used compounds, chemicals and reagents.

\begin{tabular}{|c|c|}
\hline product & supplier, location \\
\hline 2,2'-sulfonyl-bis-(3,4,6-trichlorphenol), MT4 & Sigma Aldrich, St. Louis, MO, USA \\
\hline 3-(N-morpholino)propansulfonsäure, MOPS & Sigma Aldrich, St. Louis, MO, USA \\
\hline 4',6-diamidino-2-phenylindole dihydrochlorid, DAPI & Sigma Aldrich, St. Louis, MO, USA \\
\hline acetic acid, pH 2.0 & Sigma Aldrich, St. Louis, MO, USA \\
\hline agar & Carl Roth, Karlsruhe, DE \\
\hline alkylphenylpolyethylenglycol, Triton ${ }^{\circledR}$ X 100 & Carl Roth, Karlsruhe, DE \\
\hline ampicillin & Carl Roth, Karlsruhe, DE \\
\hline bovine serum albumin, BSA & Sigma Aldrich, St. Louis, MO, USA \\
\hline calcium chloride, $\mathrm{CaCl} 2$ & Carl Roth, Karlsruhe, DE \\
\hline Chloramphenicol, ca & Carl Roth, Karlsruhe, DE \\
\hline coelenteracine & Carl Roth, Karlsruhe, DE \\
\hline DABCO & Carl Roth, Karlsruhe, DE \\
\hline diethanolamine & Carl Roth, Karlsruhe, DE \\
\hline dimethyl sulfoxide, DMSO & Sigma Aldrich, St. Louis, MO, USA \\
\hline disodiumhydrogenphosphat, $\mathrm{Na} 2 \mathrm{HPO} 4 \times \mathrm{H} 2 \mathrm{O}$ & Electran $^{\circledR}, \mathrm{DE}$ \\
\hline Escherichia coli lipopolysaccharides, LPS & Sigma Aldrich, St. Louis, MO, USA \\
\hline ethanol & Carl Roth, Karlsruhe, DE \\
\hline glucose & Sigma Aldrich, St. Louis, MO, USA \\
\hline glycerol & MP Chemicals, DE \\
\hline GSK $1210151 \mathrm{~A}$ & Sigma Aldrich, St. Louis, MO, USA \\
\hline hygromycin B gold (solution), hygro & Sigma Aldrich, St. Louis, MO, USA \\
\hline IFN-gamma recombinant human protein, IFN-g & Thermo Fisher Scientific, Oberhausen, DE \\
\hline inotilone & Cayman Chemical, Ann Arbor, MI, USA \\
\hline isopropanol & Carl Roth, Karlsruhe, DE \\
\hline kanamycin, can & Carl Roth, Karlsruhe, DE \\
\hline L-arabinose & Carl Roth, Karlsruhe, DE \\
\hline L-homoarginine & MP Biomedicals, Eschwege, DE \\
\hline lovastatin & Sigma Aldrich, St. Louis, MO, USA \\
\hline lysozyme & Sigma Aldrich, St. Louis, MO, USA \\
\hline magnesium chloride, $\mathrm{MgCl} 2$ & Sigma Aldrich, St. Louis, MO, USA \\
\hline mangan chloride, $\mathrm{MnCl} 2$ & Proanalysis, Merck, Darmstadt, DE \\
\hline methanol, $\mathrm{MeOH}$ & Carl Roth, Karlsruhe, DE \\
\hline moviol 4-88 & Carl Roth, Karlsruhe, DE \\
\hline paraformaldehyde, PFA & Sigma Aldrich, St. Louis, MO, USA \\
\hline phorbol 12-myristate 13 -acetate, PMA & Sigma Aldrich, St. Louis, MO, USA \\
\hline p-nitrophenyl phosphate & TCl, Tokio, JPN \\
\hline
\end{tabular}


Table 2.2: Continuance.

\begin{tabular}{|c|c|}
\hline product & supplier, location \\
\hline potassium acetate & Carl Roth, Karlsruhe, DE \\
\hline potassium base, $\mathrm{KOH}$ & Sigma Aldrich, St. Louis, MO, USA \\
\hline potassium chloride, $\mathrm{KCl}$ & Carl Roth, Karlsruhe, DE \\
\hline potassiumdihydrogenphosphat, $\mathrm{KH} 2 \mathrm{PO} 4$ & Carl Roth, Karlsruhe, DE \\
\hline puromycin dihydrochlorid, puro & Carl Roth, Karlsruhe, DE \\
\hline Ro $11-1464$ & Sigma Aldrich, St. Louis, MO, USA \\
\hline rosmanol & PhytoLab, Vestenbergsgreuth, DE \\
\hline sodium acetate & Sigma Aldrich, St. Louis, MO, USA \\
\hline sodium base, $\mathrm{NaOH}$ & Sigma Aldrich, St. Louis, MO, USA \\
\hline sodium chloride, $\mathrm{NaCl}$ & Carl Roth, Karlsruhe, DE \\
\hline sodium dodecyl sulphate, SDS & Sigma Aldrich, St. Louis, MO, USA \\
\hline ß-mercaptoethanol & AppliChem, Darmstadt, DE \\
\hline sucrose & Sigma Aldrich, St. Louis, MO, USA \\
\hline tetracycline hydrochloride, tet & Carl Roth, Karlsruhe, DE \\
\hline thapsigargin & Adipogen, San Diego, CA, USA \\
\hline TNF-alpha & PeproTech, Hamburg, DE \\
\hline trichostatin A, TSA & Sigma Aldrich, St. Louis, MO, USA \\
\hline Tris(hydroxymethyl)-aminomethan, TRIS & Sigma Aldrich, St. Louis, MO, USA \\
\hline TRIS-HCl & Sigma Aldrich, St. Louis, MO, USA \\
\hline tween-20 & Sigma Aldrich, St. Louis, MO, USA \\
\hline vorinostat & Sigma Aldrich, St. Louis, MO, USA \\
\hline
\end{tabular}

Table 2.3: List of used commercial kits.

\begin{tabular}{|c|c|}
\hline product & supplier, location \\
\hline Cell Line Nucleofector ${ }^{\mathrm{TM}}$ Kit V & Lonza, Basel, CHE \\
\hline CellTiter-Glo ${ }^{\circledR}$ Luminescent Cell Viability Assay & Promega, Fitchburg, WI, USA \\
\hline First Strand cDNA Synthesis Kit & Thermo Scientific ${ }^{\mathrm{TM}}$, Oberhausen, DE \\
\hline MycoAlert ${ }^{\mathrm{TM}}$ PLUS Detection Kit & Lonza, Basel, CHE \\
\hline NEBuildner ${ }^{\circledR} \mathrm{HiFi}$ DNA Assembly Cloning Kit & NEB, Ipswich, MA, USA \\
\hline NecleoBond Xtra BAC preparation kit & Macherey-Nagel, Düren, DE \\
\hline NucleoBond Gel and PCR Clean-up & Macherey-Nagel, Düren, DE \\
\hline NucleoBond Plasmid preparation kit, mini, midi & Macherey-Nagel, Düren, DE \\
\hline PEG Virus Precipitation Kit & Abcam, Cambridge, UK \\
\hline Phospha-Light ${ }^{\mathrm{TM}}$ SEAP Reporter Gene Assay System & Thermo Fisher Scientific, Oberhausen, DE \\
\hline Pierce $^{T M}$ BCA Protein Assays Kit & Thermo Fisher Scientific, Oberhausen, DE \\
\hline Quick Ligation ${ }^{\mathrm{TM}}$ Kit & NEB, Ipswich, MA, USA \\
\hline
\end{tabular}


Table 2.3: Continuance.

\begin{tabular}{ll}
\hline product & supplier, location \\
\hline RNase-Free DNase set & Qiagen, Hilden, DE \\
RNeasy Mini Kit & Qiagen, Hilden, DE \\
SEAP detection Kit, Fluorescence & Sigma Aldrich, St. Louis, MO, USA \\
\hline
\end{tabular}

Table 2.4: List of used instruments, devices and corresponding software.

\begin{tabular}{|c|c|}
\hline product & supplier, location \\
\hline 4D-Nucleofector ${ }^{\top M}$, X-Unit, 96-well Shuttle ${ }^{\top M}$ device & Lonza, Basel, CHE \\
\hline autoclave, Systec VX150 & Systec, Linden, DE \\
\hline BarTender Professional 10.1 SR 4, software (Fraunhofer IME & Citizen, Stuttgart, DE \\
\hline \multicolumn{2}{|l|}{ ScreeningPort) } \\
\hline cell counter, Scepter (Fraunhofer IME ScreeningPort) & Millipore, Burlington, MA, USA \\
\hline cell culture bench, HeraSafe HSP 12 (Fraunhofer IME Screening- & Thermo Scientific, Waltham, MA, USA \\
\hline \multicolumn{2}{|l|}{ Port) } \\
\hline cell culture bench, Herasafe KS & Thermo Scientific, Waltham, MA, USA, \\
\hline centrifuge, CL 21R & Thermo Fisher Scientific, Oberhausen, DE \\
\hline centrifuge, $5430 \mathrm{R}$ & Eppendorf, Hamburg, DE \\
\hline centrifuge, bench & Carl Roth, Karlsruhe, DE \\
\hline centrifuge, Heraeus $^{\mathrm{TM}}$ Megafuge $^{\mathrm{TM}} 16 \mathrm{R}$ (Fraunhofer IME & Eppendorf, Hamburg, DE \\
\hline \multicolumn{2}{|l|}{ ScreeningPort) } \\
\hline centrifuge, Heraeus ${ }^{T M}$ Megafuge $\mathrm{e}^{\mathrm{TM}} 40 \mathrm{R}$ & Thermo Fisher Scientific, Oberhausen, DE \\
\hline chemical cabinet & Mc6, Das Laborsystem, DE \\
\hline ChemiDoc ${ }^{\mathrm{TM}}$ MP Imaging system & Bio-Rad, Hercules, CA, USA \\
\hline Echo 550 Liquid Handler (Fraunhofer IME ScreeningPort) & Labcyte, San Jose, CA, USA \\
\hline $\begin{array}{l}\text { Echo Plate Reformat 1.5.6, software (Fraunhofer IME Scree- } \\
\text { ningPort) }\end{array}$ & Labcyte, San Jose, CA, USA \\
\hline EnSpire ${ }^{\mathrm{TM}}$ Multimode Plate Reader & PerkinElmer, Waltham, MA, USA \\
\hline EnVision ${ }^{\circledR}$ HTS Plate Reader (Fraunhofer IME ScreeningPort) & PerkinElmer, Waltham, MA, USA \\
\hline extra cold fridge, HFU500TV60 (Fraunhofer IME ScreeningPort) & Thermo Scientific, Waltham, MA, USA \\
\hline freezer, $-80^{\circ} \mathrm{C}$, HERAfreeze HFU T Series & Thermo Fisher Scientific, Oberhausen, DE \\
\hline freezers, $-20^{\circ} \mathrm{C}$ & Liebherr, Bulle, CHE \\
\hline fridges, $4^{\circ} \mathrm{C}$ & Liebherr, Bulle, CHE \\
\hline Gene Pulser Xcell ${ }^{\mathrm{TM}}$ electroporation system & Bio-Rad, Hercules, CA, USA \\
\hline heat cabinet, HeraTherm IGS60 (Fraunhofer IME ScreeningPort) & Thermo Electron LED, Langenselbold, DE \\
\hline heat cabinet, HeraTherm Oven & Thermo Scientific, Waltham, MA, USA \\
\hline ice machine, AF 103 & Scotsman, Milano, IT \\
\hline incubator, bacteria plates & Thermo Scientific, Waltham, MA, USA \\
\hline incubator, bacteria, MAXQ 8000 & Thermo Fisher Scientific, Oberhausen, DE \\
\hline
\end{tabular}


Table 2.4: Continuance.

\begin{tabular}{|c|c|}
\hline product & supplier, location \\
\hline 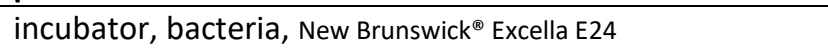 & Eppendorf, Hamburg, DE \\
\hline incubator, cell culture plate, Cytomat 6001 (Fraunhofer IME & Liconic, Mauren, LIE \\
\hline \multicolumn{2}{|l|}{ ScreeningPort) } \\
\hline incubator, cell culture, HeraCell 240 (Fraunhofer IME Screening- & Thermo Scientific, Waltham, MA, USA \\
\hline \multicolumn{2}{|l|}{ Port) } \\
\hline incubator, cell culture, New Brunswick'm GALAXY® 170 S & Eppendorf, Hamburg, DE \\
\hline JANUS Mini Plattform, MDT-Arm (Fraunhofer IME ScreeningPort) & PerkinElmer, Waltham, MA, USA \\
\hline label print system, CLP-631 (Fraunhofer IME ScreeningPort) & Citizen, Stuttgart, DE \\
\hline magnetic stirrer & NeoLab, Heidelberg, DE \\
\hline microscope, 6V30W1AF (Fraunhofer IME ScreeningPort) & VWR, Radnor, PE, USA \\
\hline microscope, confocal fluorescent, AxioVison v4.8 & Carl Zeiss, Oberkochen, DE \\
\hline microscope, Observer Z1 with AxioCam MRC camera & Carl Zeiss, Oberkochen, DE \\
\hline microwaves & Bosch, Gerlingen, DE; Sharp, Osaka, JPN \\
\hline nitrogen system, LS-600 & Taylor Wharton, Osaka, JPN \\
\hline PCR cycler, 2720 Thermal Cycler & Thermo Fisher Scientific, Oberhausen, DE \\
\hline PCR cycler, Vapo protect PCR Cycler & Eppendorf, Hamburg, DE \\
\hline pH-meter, pH Fix 4.5-10.0 & Carl Roth, Karlsruhr, DE \\
\hline plate washer, ELX405 (Fraunhofer IME ScreeningPort) & BioTeck, Nogarazza, IT \\
\hline power supply, PEQ power 300V & Peqlab, VWR, Radnor, PE, USA \\
\hline qPCR cabinet, UVP PCR Workstation & Analytik Jena, Jena, DE \\
\hline qPCR cycler, CFX96 Touch Real-Time PCR Detection System & Bio-Rad, Hercules, CA, USA \\
\hline qPCR cycler, Quantstudio 12K Flex system & Thermo Fisher Scientific, Oberhausen, DE \\
\hline roller, su 1400 & SunLab, Hamburg, DE \\
\hline scale, s-64 & Denver Instrument, Bohemia, NY, USA \\
\hline scale, Scout Pro $6000 \mathrm{~g}$ & Ohaus, Parsippany, NJ, USA \\
\hline shaker, PSS-10L & NeoLab, Heidelberg, DE \\
\hline spectrophotometer, Nanodrop ${ }^{\mathrm{TM}} 2000$ & Thermo Fisher Scientific, Oberhausen, DE \\
\hline ThermoMix Pro & Cell Media, DE \\
\hline ThermoMixer ${ }^{\circledast} \mathrm{C}$ & Eppendorf, Hamburg, DE \\
\hline Trans-Blot ${ }^{\circledR}$ Turbo $^{\text {TM }}$ System, semi-dry & Bio-Rad, Hercules, CA, USA \\
\hline vacuum pump, bench & Fischer Scientific, Oberhausen, DE \\
\hline vacuum pump, cell culture, $2511 c-02$ & Thermo Scientific, Waltham, MA, USA \\
\hline vacuum pump, cell culture, BrandTech ${ }^{T M}$ Vacuubrand $d^{T M}$ BVC & Fischer Scientific, Oberhausen, DE \\
\hline vortex, Genie 2 & Scientific Industries, Bohemia, NY, USA \\
\hline Wallac EnVision Manager 1,12, software (Fraunhofer IME & PerkinElmer, Waltham, MA, USA \\
\hline ScreeningPort) & \\
\hline water bath, GFL 1086 & GFL, Burgwedel, DE \\
\hline
\end{tabular}


Table 2.4: Continuance.

\begin{tabular}{ll}
\hline product & supplier, location \\
\hline water bath, GFL 11817318 K (Fraunhofer IME ScreeningPort) & GFL, Burgwedel, DE \\
water destillator, Mlli-Q ${ }^{\circledR}$ Integral & Merck Millipore, Burlington, MA, USA \\
WinPREP 4.10.0.54, software (Fraunhofer IME ScreeningPort) & PerkinElmer, Waltham, MA, USA \\
ZOE Fluorescent cell imager & Bio-Rad, Hercules, CA, USA \\
\hline
\end{tabular}

\subsection{Stock solutions and buffers}

The used stock solutions, buffers and the corresponding ingredients are listed below (Table 2.5).

Table 2.5: List of used stock solutions and buffers.

\begin{tabular}{|c|c|}
\hline solution / buffer & ingredients \\
\hline $\mathrm{CaCl}_{2}, 1 \mathrm{M}$ & $1 \mathrm{M} \mathrm{CaCl}_{2}$ in $\mathrm{dd} \mathrm{H}_{2} \mathrm{O}$ \\
\hline EDTA, $0.5 \mathrm{M}, \mathrm{pH} 7.6$ & $0.5 \mathrm{M}$ EDTA $(\mathrm{w} / \mathrm{v}), \mathrm{pH}$ adjusted with $\mathrm{NaOH}$ \\
\hline $\mathrm{KCl}, 1 \mathrm{M}$ & $1 \mathrm{M} \mathrm{KCl}$ in dd $\mathrm{H}_{2} \mathrm{O}$ \\
\hline $\mathrm{MgCl}_{2}, 1 \mathrm{M}$ & $1 \mathrm{M} \mathrm{MgCl}_{2}$ in $\mathrm{ddd}_{2} \mathrm{O}$ \\
\hline $\mathrm{MnCl}_{2}, 1 \mathrm{M}$ & $1 \mathrm{M} \mathrm{MnCl}_{2}$ in $\mathrm{dd} \mathrm{H}_{2} \mathrm{O}$ \\
\hline $\mathrm{NaCl}, 5 \mathrm{M}$ & $5 \mathrm{M} \mathrm{NaCl}$ in $\mathrm{dd} \mathrm{H}_{2} \mathrm{O}$ \\
\hline PBS, pH 7.4 & $137 \mathrm{mM} \mathrm{NaCl}, 2.6 \mathrm{mM} \mathrm{KCl}, 8.1 \mathrm{mM} \mathrm{Na}_{2} \mathrm{HPO}_{4}, 1.5 \mathrm{mM} \mathrm{KH}_{2} \mathrm{PO}_{4}, \mathrm{pH} 7.4$ \\
\hline TRIS-HCl, $1 \mathrm{M}, \mathrm{pH} 8.0$ & $1 \mathrm{M}$ Tris (w/v), $\mathrm{pH}$ adjusted with $\mathrm{HCl}$ \\
\hline
\end{tabular}

\subsection{DNA ladders}

The used DNA ladders are shown below (Fig.2-1).

A

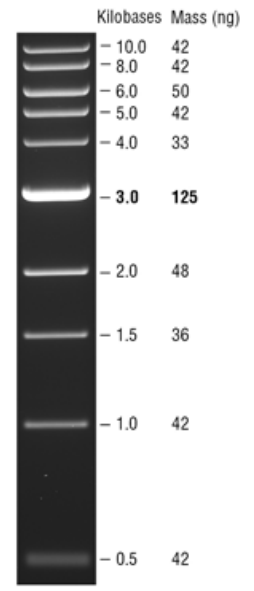

B

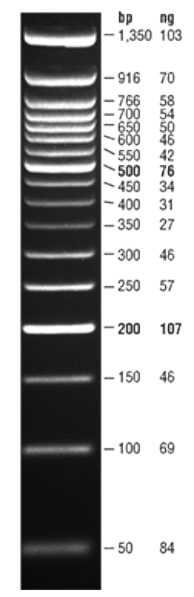

C

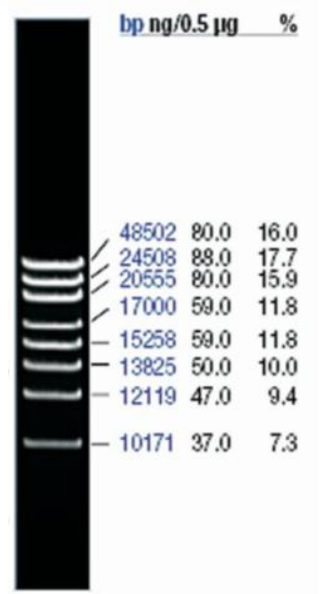

Figure 2-1: DNA ladders used in agarose gel electrophoresis.

A: Quick-Load ${ }^{\circledR} 1$ kb DNA Ladder (NEB, cat\# N0468) on a $0.8 \%$ TAE agarose gel. Mass values are for 0.5 $\mu \mathrm{g} /$ gel lane. B: Quick-Load ${ }^{\circledR}$ purple 50 bp DNA Ladder (NEB, cat\# N0556) on a $3 \%$ TAE agarose gel. Mass values are for $1 \mu \mathrm{g} /$ lane. C: GeneRuler ${ }^{\mathrm{TM}}$ high range DNA ladder (Thermo Scientific ${ }^{\mathrm{TM}}$, cat\# 11843993) on a $0.4 \% \mathrm{TAE}$ agarose gel, $5 \mathrm{~V} / \mathrm{cm}, 1.5 \mathrm{~h}$. Mass values are for $0.4 \mu \mathrm{g} / \mathrm{lane}$. 


\subsection{Protein ladders}

The used protein ladders are shown below (Fig.2-2).

A

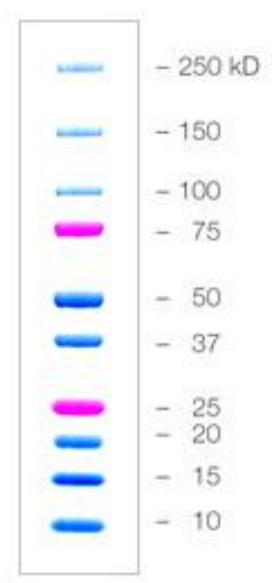

B

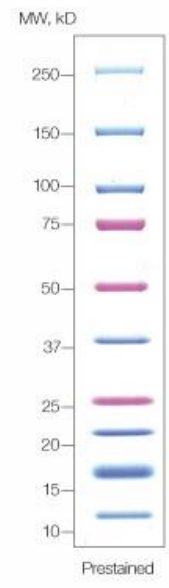

Figure 2-2: Protein ladders used in SDS-PAGE.

Protein ladders Precision Plus Protein ${ }^{\mathrm{TM}}$ Dual Color Standard (Bio-Rad, cat\# 1610374) (A) and Precision Plus Protein ${ }^{\text {TM }}$ WesternC ${ }^{\text {TM }}$ Standard (Bio-Rad, cat\# 1610385) (B) were used for protein molecular weight estimation on SDS-PAGE gels and western blots.

\subsection{Antibodies}

\subsubsection{Primary antibodies}

The following primary antibodies were used for Western Blotting and immunofluorescence staining (Table 2.6).

Table 2.6: Primary antibodies used for Western Blotting (WB) and immunofluorescence (IF) analysis.

\begin{tabular}{llllllll}
\hline antibody & monoclonal & clone / & species & \multicolumn{2}{c}{ dilution } & reference \\
\cline { 5 - 6 } & / polyclonal & cat\# & & WB & IF & \\
\hline anti-c-myc & poly & C3956 & rabbit & $1: 500$ & $1: 100$ & Sigma Aldrich \\
anti-GRP78 & mono & sc-376768 & mouse & $1: 1,000$ & - & Santa Cruz Biotechnology \\
anti-GAPDH & mono & $14 C 10$ & rabbit & $1: 1,000$ & - & Cell Signaling Technology \\
\hline
\end{tabular}

\subsubsection{Secondary antibodies}

The following secondary antibodies were used for Western Blotting and immunofluorescence staining (Table 2.7 ).

Table 2.7: Secondary antibodies used for protein detection.

HRP (horseradish peroxidase) and Alexa conjugated antibodies were used for Western Blotting and immunofluorescence staining, respectively.

\begin{tabular}{lllll}
\hline antigen & species & conjugation & dilution & reference, cat\# \\
\hline anti-rabbit IgG & goat & HRP & $1: 15,000$ & Sigma Aldrich, A9169 \\
anti-rabbit IgG & donkey & Alexa 555 & $1: 1,000$ & Thermo Fisher Scientific, A-31572
\end{tabular}


Table 2.7: Continuance.

\begin{tabular}{lllll}
\hline antigen & species & conjugation & dilution & reference, cat\# \\
\hline anti-mouse IgG & rabbit & HRP & $1: 30,000$ & Sigma Aldrich, A9044 \\
\hline
\end{tabular}

\subsection{Bacterial strains}

The bacterial strains used for cloning are listed in Table 2.8.

Table 2.8: Escherichia coli (E. coli) bacterial strains used for plasmid and BAC cloning.

\begin{tabular}{|c|c|c|}
\hline strain & genotype & reference \\
\hline $\mathrm{DH} 5 \alpha$ & $\begin{array}{l}\text { F- supE44 } \triangle \text { lacU169 } \varphi 80 \text { lacZDM15 hsdR17 recA1 endA1 gyrA96 } \\
\text { thi-1 relA1 }\end{array}$ & Bachmann 204 \\
\hline SCS110 & $\begin{array}{l}\text { rpsL (Str) thr leu endA thi-1 lacY galK galT ara tonA tsx dam } \\
\text { dcm supE44 } \triangle \text { (lac-proAB) [F traD36 proAB lacla Z } \triangle M 15]\end{array}$ & Agilent, cat\# 200247 \\
\hline DH10B & $\begin{array}{l}\text { F- mcrA } \Delta \text { (mrr-hsdRMS-mcrBC) } \varphi 80 \text { lacZ } \Delta M 15 \Delta \text { lacX74 recA1 } \\
\text { endA1 araD139 } \Delta \text { (ara-leu)7697 galU galK } \lambda-\operatorname{rpsL(StrR)~nupG~}\end{array}$ & BACPAC Chori ${ }^{205}$ \\
\hline PIR1 & $\begin{array}{l}\text { F- } \Delta \text { lac169 rpoS(Am) robA1 creC510 hsdR514 endA recA1 uidA } \\
\text { ( } \Delta \text { Mlul) ::pir-116 }\end{array}$ & $\begin{array}{l}\text { Thermo Fisher Scientific, } \\
\text { cat\# C1010-10 }\end{array}$ \\
\hline
\end{tabular}

\subsection{Cell lines}

The used wild type and generated reporter cell lines are listed below (Table 2.9).

Table 2.9: Wild type and generated reporter cell lines.

*THP-1 eGFP reporter cells were kindly provided by the working group of Prof. Brüne, Institute of Biochemistry I Pathobiochemistry, Goethe University, Frankfurt am Main.

\begin{tabular}{llll}
\hline cell line & origin & $\begin{array}{l}\text { expressed reporter } \\
\text { genes }\end{array}$ & source \\
\hline HEK 293T & human embryonic & non (wild type cell line) & $\begin{array}{l}\text { ATCC: CRL-11268, } \\
\text { Leibniz Institute DSMz, } \\
\text { cat\# ACC 635 }\end{array}$ \\
kidney & & & ATCC: TIB-202, \\
& monocyte-like & non (wild type cell line) & Leibniz Institute DSMz, \\
& cells & & cat\# ACC 16 \\
HEK 239T-G2P & & & this study \\
HEK 293T-G2P-SEAP & generated & eGFP & this study \\
HEK 293T-G2P-GLuc & generated & eGFP, SEAP-myc & this study \\
THP-1 eGFP & generated & eGFP, GLuc-myc & $*$ \\
THP-1 CMV::GLuc-CEBPD::SEAP & generated & eGFP & this study \\
THP-1 CMV::SEAP-CEBPD::GLuc & generated & eGFP, SEAP-myc, GLuc-myc & this study \\
\hline
\end{tabular}

eGFP: enhanced green fluorescent protein; G2P: eGFP linked via T2A self-cleavage peptide to puromycin resistance gene; SEAP: secreted alkaline phosphatase; GLuc: Gaussia luciferase; CMV: cytomegalovirus constitutive promoter; CEBPD: CCAAT/enhanced binding protein delta promoter. 


\subsection{Oligonucleotides}

The used primers for cloning (Table 2.10) and sequencing (Table 2.11) are summarized below.

Table 2.10: Sequences of oligonucleotides used for cloning.

F: forward; R: reverse. Restriction sites are underlined.

\begin{tabular}{|c|c|}
\hline oligonucleotide & sequence in $5^{\prime}$ to $3^{\prime}$ direction \\
\hline F: G2P_BsaBI & AGACAGGATGAGGATCGTTTCGCATGGAGAGCGACGAGAGC \\
\hline R: G2P_BstBI & GGTCATTTCGAACCCCAGAGTCCCGTCAGGCACCGGGCTTGCG \\
\hline F: Gaussia_myc & AGGGGATCCAGCCACCATGG \\
\hline R: Gaussia_myc_HindIII & CTTAAGCTTTTACAGATCCTCTTCTGAGATGAGTTTTTGTTCGTCACCACCGG \\
\hline F: uni_Nhel & GGAGACCCAAGCTGGCTAGC \\
\hline R: myc_NLS_mCherry & $\begin{array}{l}\text { TTTGGATCCCGGGCCCGCGGTACCCTGTCCAACTTGACCCTCTTGGCAG- } \\
\text { CAGGCAGATCCTCTTCTGAGATG }\end{array}$ \\
\hline F: KfII-CMV-Space & CACCTCCCAACCCCGAGGGGACCCGACAGGGTTGACATTGATTATTGACTAGTTAT \\
\hline R: Space-SPg & AGGGCAAACAGAACTTTGACTCCCATATTTCGATAAGCCAGTAAGCAGT \\
\hline F: Space_SPg & CCACTGCTTACTGGCTTATCGAAATATGGGAGTCAAAGTTCTGTTTGCCCTGAT \\
\hline R: BGH poly EcoRI & CTGCAGGAATTCGATAGCCATAGAGCCCACCGCATCCCCAGCAT \\
\hline F: Space_SPs & CCACTGCTTACTGGCTTATCGAAATATGCTGCTGCTGCTGCTGCTGCTGGGCCT \\
\hline R: Space_SPs & AGGCCCAGCAGCAGCAGCAGCAGCAGCATATTTTGATAAGCCAGTAAGCAGT \\
\hline F: Xhol SV40 poly & TCGACCTCGAGGCAAACTTGTTTATTGCAGCTTATAATGGT \\
\hline R: SV40 poly BGH poly & CTGGGGATGCGGTGGGCTCTATGGCACAGACATGATAAGATACATTGATGA \\
\hline F: gaussia backbone-CEBPD & CAAGGCAAGGCTTGACCGACGGAGTGTCATTCCCAGCAGCGCAGCGC \\
\hline R: SEAP-myc-CEBPD & GCAGCAGCAGCAGCATGGCGGCGTCGGGCCGGGC \\
\hline F: BGH poly SV40 poly & TCATCAATGTATCTTATCATGTCTGTGCCATAGAGCCCACCGCATCCCCAGCAT \\
\hline R: Acc65I CEBPD & TTAAAGGTACCGAGCTGGAGTGTCATTCCCAGCAGCGCA \\
\hline F: Xhol_pA_Gluc & TCTTACTCGAGGCCATAGAGCCCACCGCAT \\
\hline R: EcoRV_CMV & TCTGCAGATATCGACATTGATTATTGA \\
\hline \multirow[t]{2}{*}{ F: T2A_hygro } & AGGGCCGGGATTCTCCTCCACGTCACCGCATGTTAGAA- \\
\hline & GACTTCCTCTGCCCTCTTCCTTTGCCCTCGGACGAGT \\
\hline R: bGH_PGK & GGGATGCGGTGGGCTCTATGGCTACCGGGTAGGGGAGGCGCTTTTTCCCAA \\
\hline F: pcDNA_G2H & TTTAAACGGGCCCTCTAGACTCGAGGAAGGGTTCCGCAAGCTCTAG \\
\hline R: NLS_bGH & AAGTTGGACTAGGGGGATCAATTCTCTAGAGCTCGCTGA \\
\hline F: bGH_NLS_cGFP & TCAGCGAGCTCTAGAGAATTGATCCCCCTAGTCCAACTTGACCCTCTT \\
\hline R: GFP_NLS & $\begin{array}{l}\text { ATGTTAGAAGACTTCCTCTGCCCTCGTCCAACTTGACCCTCTTGGCAGCAGGGCGA- } \\
\text { GATCCGGTGGAGCCGGGT }\end{array}$ \\
\hline R: T2A_cGFP & ATGCGGTGACGTGGAGGAGAATCCCGGCCCTATGGAGAGCGACGAGA \\
\hline F: pcDNA_Prp & CCCAAGCTGGCTAGCATGGCGAACCTTAGC \\
\hline R: SV40_Poly_pcDNA & GAACCCTTCCTCGAGTAAGATACATTGATG \\
\hline F: Nhe_Prp_SEAP & CTGGCTAGCATGGCGAACCTTAGCTA \\
\hline R: Xho_SV40 & CCTTCCTCGAGTAAGATACATTGATGA \\
\hline F: Bmtl_Gluc & $\begin{array}{l}\text { AGGAGTTTACGTCCAGCCAAGCTAGCGTTGACATTGATTATTGACTAGTTATTAA- } \\
\text { TAGTAATCAATTACGGG }\end{array}$ \\
\hline R: Prp_Sacl & CACAGTCGAGGCTGATCAGCGAGCTCATGGCGAACCTTAGCTAC \\
\hline F: PCR_CEBPD_cassette & АTACTCATACTCTTCCTTTTTTCAATATTGGAGTGTCATTCCCAGCAGCGCAGCGC \\
\hline R: PCR_CEBPD_cassette & GCAGCCAGTAGCTAAGGTTCGCCATGGCGGCGTCGGGCCGGGC \\
\hline
\end{tabular}


Table 2.11: Sequences of oligonucleotides used for sequencing.

F: forward; R: reverse.

\begin{tabular}{|c|c|}
\hline oligonucleotide & sequence in $5^{\prime}$ to $3^{\prime}$ direction \\
\hline F: pcDNA_G2P_sv40 & TTCCAGAAGTAGTGAGAAGGC \\
\hline R: pcDNA_G2P & AGGTTGGGCGTCGCTTGGTC \\
\hline F: seq_Gaussia & ATATCTGCAGAATTCCACCA \\
\hline F: seq_CMV_TB & GAGGTCTATATAAGCAGAGC \\
\hline R: BGH_Poly & AACAGATGGCTGGCAACTAG \\
\hline F: seq_CMV & GAGGTCTATATAAGCAGAGC \\
\hline$R$ : seq-mCherry & GCATGAACTCCTTGATGATG \\
\hline seq_CEBPD_viral_2 & CCATTCCTGCACCAGATTCT \\
\hline seq_CEBPD_viral_3 & GACATCGCTACGCAGCTCATCT \\
\hline F: seq_pSEW_GFP & GATATTCACCATTATCGTTT \\
\hline seq_CEBPD_viral_5 & TGTCGACGATCGCCTCGCCT \\
\hline seq_CEBPD_viral_4 & AATTGCATGAAGAATCTGCT \\
\hline R: qPCR_SEAP_WT & CGCCCAGGAAGATGATGAGG \\
\hline seq_CEBPD_viral_6 & ACAGGTCGATCTGTGTGTGGACT \\
\hline R:PCR_SEAP_space & ACGCCTAACCCTAAGCAGAT \\
\hline F: qPCR_Gaussia & CCCACCGAGAACAACGAAGA \\
\hline R: qPCR_Gaussia & GGGCATTGGCTTCCATCTCT \\
\hline F: pcDNA_G2H & TTTAAACGGGCCCTCTAGACTCGAGGAAGGGTTCCGCAAGCTCTAG \\
\hline R: bGH_PGK & GGGATGCGGTGGGCTCTATGGCTACCGGGTAGGGGAGGCGCTTTTCCCAA \\
\hline R: T2A_cGFP & ATGCGGTGACGTGGAGGAGAATCCCGGCCCTATGGAGAGCGACGAGA \\
\hline F: T2A_hygro & $\begin{array}{l}\text { AGGGCCGGGATTCTCCTCCACGTCACCGCATGTTAGAAGACTTCCTCTGCCCTCTTCCTTT- } \\
\text { GCCCTCGGACGAGT }\end{array}$ \\
\hline R: SV40_Poly_pcDNA & GAACCCTTCCTCGAGTAAGATACATTGATG \\
\hline F: qPCR_SEAP_WT & CAGTTGAGGAGGAGAACCCG \\
\hline F: BAC_PCR_7 & CCTCCAGCAGCCTCTACAGT \\
\hline R: BAC_PCR_2 & GGAGTGTCATTCCCAGCAGC \\
\hline R: BAC_PCR_1 & ACTGTAGAGGCTGCTGGAGG \\
\hline
\end{tabular}

\subsection{Plasmids}

The already available plasmids, listed in the Table 2.12 , served as template vectors for the generated plasmids, listed in the Table 2.13. The used SEAP sequence corresponds to this from GenBank (acc\# U89937). BAC clone CH17-293N3 (BACPAC services, CHORI) served as a genetic source of CEBPD target promoter sequence. Plasmid maps of used and generated plasmids are shown in chapter 8.9 (Fig.8-17 - Fig.8-42).

Table 2.12: List of available plasmids.

a: working group of Prof. Starzinski-Powitz, Institute of Cell Biology and Neuroscience, Goethe University, Frankfurt am Main

b: working group of Prof. Brüne, Institute of Biochemistry I - Pathobiochemistry, Goethe University, Frankfurt am Main

c: Dr. Grote, IncRNA Research in cardio-pulmonary development, Goethe University, Frankfurt am Main

\begin{tabular}{lllcc}
\hline construct & $\begin{array}{l}\text { description/encoded } \\
\text { genes }\end{array}$ & backbone & reference / source & $\begin{array}{l}\text { plasmid } \\
\text { map Fig. }\end{array}$ \\
\hline pcDNA3.1(-) & backbone vector & - & a & $8-17$ \\
wt-SEAP-myc & wild type SEAP, myc-tagged & pcDNA3.1(-) & a & $8-18$
\end{tabular}


Table 2.12: Continuance.

\begin{tabular}{|c|c|c|c|c|}
\hline construct & $\begin{array}{l}\text { description/encoded } \\
\text { genes }\end{array}$ & backbone & reference / source & $\begin{array}{l}\text { plasmid } \\
\text { map Fig. }\end{array}$ \\
\hline$\triangle$ SP-SEAP-myc & wt-SEAP-myc, deleted SP & pcDNA3.1(-) & $\mathrm{a}$ & 8-19 \\
\hline shrew-1- $\Delta$ SP-SEAP-myc & $\begin{array}{l}\text { shrew-1 SP fused to } \triangle S P \text { - } \\
\text { SEAP-myc }\end{array}$ & pcDNA3.1(-) & a & $8-20$ \\
\hline Prl- $\triangle$ SP-SEAP-myc & $\begin{array}{l}\text { Prl SP fused to } \triangle \text { SP-SEAP- } \\
\text { myc }\end{array}$ & pcDNA3.1(-) & a & $8-21$ \\
\hline $\operatorname{Prp}-\triangle \mathrm{SP}-\mathrm{SEAP}-\mathrm{myc}$ & $\begin{array}{l}\text { Prp SP fused to } \triangle \text { SP-SEAP- } \\
\text { myc }\end{array}$ & pcDNA3.1(-) & a & $8-22$ \\
\hline pSEW-eGFP & eGFP fluorescent protein & pSEW & $b$ & $8-23$ \\
\hline pX335A-G2P & G2P construct & pX335A & c & $8-24$ \\
\hline pR6k(hygro)-bglobin & $\begin{array}{l}\text { hygromycin resistance } \\
\text { gene }\end{array}$ & pR6k & c & $8-25$ \\
\hline pSC101-gbaA & gbaA recombinases & pSC101 & c & $8-26$ \\
\hline pmCherry-N1 & $\begin{array}{l}\text { mCherry fluorescent pro- } \\
\text { tein }\end{array}$ & not specified & Clontech, cat\# 632523 & $8-27$ \\
\hline pCMV-Gaussia & Gaussia luciferase & not specified & $\begin{array}{l}\text { Thermo Fisher, cat\# } \\
16147\end{array}$ & $8-28$ \\
\hline $\mathrm{CH} 17-293 \mathrm{~N} 3$ & $\begin{array}{l}\text { CEBPD-expressing BAC } \\
\text { clone }\end{array}$ & pBACGK1.1 & BACPAC CHORI, ${ }^{205}$ & none \\
\hline
\end{tabular}

Table 2.13: List of generated plasmids.

Some of the listed plasmids were generated over multiple steps (helping plasmids), as described in each of the corresponding cloning strategies (chapter 8.8).

*Plasmids generated by Holger Jordan, Fraunhofer IME-TMP, Frankfurt am Main.

\begin{tabular}{|c|c|c|c|c|}
\hline construct & description & reference & chapter & $\begin{array}{l}\text { plasmid } \\
\text { map Fig. }\end{array}$ \\
\hline pcDNA3.1(-)-G2P & $\begin{array}{l}\text { copGFP coupled via T2A to } \\
\text { puromycin resistance }\end{array}$ & this study & 8.8 .1 & $8-29$ \\
\hline pcDNA3.1(-)-G2P-SEAP-myc & wt SEAP, myc-tagged & this study & 8.8 .1 & $8-30$ \\
\hline pcDNA3.1(-)-G2P-GLuc-myc & Gaussia, myc-tagged & this study & 8.8 .1 & $8-31$ \\
\hline pmCherry-wt-SEAP-myc-NLS & wt SEAP-myc, NLS-tagged & this study* & 8.8 .2 & $8-32$ \\
\hline pmCherry- $\triangle$ SEAP-myc-NLS & $\Delta$ SEAP-myc, NLS-tagged & this study* & 8.8 .2 & $8-33$ \\
\hline $\begin{array}{l}\text { pmCherry-shrew-1-SEAP-myc- } \\
\text { NLS }\end{array}$ & $\begin{array}{l}\text { shrew-1 SEAP-myc, NLS- } \\
\text { tagged }\end{array}$ & this study* & 8.8 .2 & $8-34$ \\
\hline pmCherry-Prp-SEAP-myc-NLS & Prp SEAP-myc, NLS-tagged & this study* & 8.8 .2 & $8-35$ \\
\hline pmCherry-PrI-SEAP-myc-NLS & Prl SEAP-myc, NLS-tagged & this study* & 8.8 .2 & $8-36$ \\
\hline $\begin{array}{l}\text { pSEW-eGFP-CMV-GLuc-myc- } \\
\text { CEBPD-SEAP-myc }\end{array}$ & wt Gaussia-myc, wt SEAP-myc & this study & 8.8 .3 & $8-37$ \\
\hline $\begin{array}{l}\text { pSEW-eGFP-CMV-SEAP-myc- } \\
\text { CEBPD-GLuc-myc }\end{array}$ & wt Gaussia-myc, wt SEAP-myc & this study & 8.8 .3 & $8-38$ \\
\hline
\end{tabular}


Table 2.13: Continuance.

\begin{tabular}{lllcc}
\hline construct & description & reference & chapter & $\begin{array}{c}\text { plasmid } \\
\text { map Fig. }\end{array}$ \\
\hline pcDNA3.1(-)-H2G-NLS-CMV-GLuc- & hygromycin resistance fused & this study & 8.8 .4 & $8-39$ \\
myc & $\begin{array}{l}\text { via T2A to copGFP-NLS, wt } \\
\text { Gaussia-myc }\end{array}$ & & \\
& Prp-SEAP-myc-NLS, H2G- & this study & 8.8 .4 & $8-40$ \\
pcDNA3.1(-)-Prp-SEAP-myc-NLS- & NLS, wt Gaussia-myc & & $8-41$ \\
H2G-NLS-CMV-GLuc-myc & Prp-SEAP-myc-NLS, H2G- & this study & 8.8 .5 & $8-42$ \\
pR6k-Prp-SEAP-myc-NLS-H2G-NLS- & & & \\
CMV-GLuc-myc & NLS, wt Gaussia-myc & this study & 8.8 .6 & \\
pcDNA3.1(-)-CEBPD-Prp-SEAP-myc- & Prp-SEAP-myc-NLS, H2G- & & \\
NLS-H2G-NLS-CMV-GLuc-myc & NLS,wt Gaussia-myc & & \\
\hline
\end{tabular}

\subsection{Software}

Clone Manager Professional 9, Scientific \& Educational Software

EndNote X8, Thomson ISI ResearchSoft

GraphPad Prism 8.0.0, Statistical Scientific Software, GraphPad

Image Lab, Life Science Research, Bio-Rad

Microsoft Office 2013, Microsoft

SnapGene ${ }^{\circledR}$ Viewer 3.2.1, gene maps generation, GSL Biotech LLC, Chicago, IL, USA

TIBCO Spotfire ${ }^{\circledR}$, data visualization and analytics, TIBCO Software Inc., Palo Alto, CA, USA

Zeiss microscope software AxioVison v4.8

\subsection{Internet sources}

BLAST $^{\circledR}$, Basic Local Alignment Search Tool, NCBI

(https://blast.ncbi.nlm.nih.gov/Blast.cgi)

Cleavage Close to the End of DNA Fragments, usage guidelines, NEB

(https://international.neb.com/tools-and-resources/usage-guidelines/cleavage-closeto-the-end-of-dna-fragments) 
Harmonizome database, Oxford

(http://amp.pharm.mssm.edu/Harmonizome/gene/CEBPD)

Integrity: knowledge-based drug discovery and development database

(https://integrity.clarivate.com)

National Center for Biotechnology Information (NCBI) and its resources

(http://www.ncbi.nlm.nih.gov/)

NEBuildner ${ }^{\circledast}$ Assembly Tool, v2.2.5, planning of assembly cloning strategy, NEB

(http://nebuilder.neb.com/\#!/)

NEBioCalculator ${ }^{\mathrm{TM}}$, v1.10.0, planning of ligation reaction, NEB

(http://nebiocalculator.neb.com/\#!/ligation)

NEBcloner ${ }^{\circledR}$, v1.3.13, planning of restriction enzyme double digestion, NEB

(https://nebcloner.neb.com/\#!/redigest)

Optimizing Restriction Endonuclease Reactions, tools and resources, NEB

(https://www.neb.com/tools-and-resources/usage-guidelines/optimizing-restriction-

endonuclease-reactions)

Poly(A) Signal Miner, prediction of poly(A) signals in human DNA sequences, Liu at al., 2003 (http://dnafsminer.bic.nus.edu.sg/PolyA.html)

Primer-BLAST, primer design tool, Coulouris et al., 2012

(https://www.ncbi.nlm.nih.gov/tools/primer-blast/)

Reverse complement, The Sequence Manipulation Suite, Paul Stothard

(http://www.bioinformatics.org/sms/rev_comp.html)

Tm Calculator, v1.12.0, NEB

(http://tmcalculator.neb.com/\#!/main)

UCSC Genome Browser, on human Dec. 2013 (GRCh38/hg38) Assembly

(https://genome.ucsc) 


\subsection{Determination of DNA and RNA concentration}

DNA and RNA concentrations were determined using the Nanodrop 2000C spectrophotometer, by measuring the absorption at the wavelength of $260 \mathrm{~nm}$. The DNAse and RNAse-free water was used as a reference.

\subsection{Generation of competent E.coli}

For the uptake of plasmid DNA (transformation), E. coli DH5 $\alpha$ and $\mathrm{DH} 10 \mathrm{~B}$ were made chemo-competent following the protocol below.

- inoculation of a $5 \mathrm{~mL} \mathrm{LB}$ medium with incompetent E-coli on a shaker at $37^{\circ} \mathrm{C}$, overnight

- inoculation of $99 \mathrm{~mL} \mathrm{LB}$ medium with $1 \mathrm{~mL}$ of the overnight culture

- incubation on a shaker at $37^{\circ} \mathrm{C}$ till $\mathrm{OD}_{600}=0.4-0.5$

- centrifugation of bacterial suspension for $5 \mathrm{~min}$, at $3,000 \times \mathrm{g}$ and $4^{\circ} \mathrm{C}$, discard supernatant

- pellet resuspension in $30 \mathrm{~mL}$ of ice-cold TFB-I buffer

- incubation of bacterial suspension for $30 \mathrm{~min}$ on ice

- centrifugation of bacterial suspension for $5 \mathrm{~min}$, at $3,000 \times \mathrm{g}$ and $4^{\circ} \mathrm{C}$, discard supernatant

- pellet resuspension in $4 \mathrm{~mL}$ of ice-cold TFB-II buffer

- aliquot the bacteria suspension (app. $100 \mu \mathrm{L}$ aliquots) in $1.5 \mathrm{~mL}$ reaction tubes

- shock freezing of aliquots in liquid nitrogen and storage at $-80^{\circ} \mathrm{C}$

TFB-I buffer

$10 \mathrm{mM} \quad \mathrm{KCl}$

$50 \mathrm{mM} \quad \mathrm{MnCl}_{2}$

$20 \mathrm{mM}$ potassium acetate

$10 \mathrm{mM} \quad \mathrm{CaCl}_{2}$

$15 \%(v / v)$ glycerol

sterile filtered and stored at $4^{\circ} \mathrm{C}$
TFB-II buffer

$19 \mathrm{mM} \quad \mathrm{MOPS}$
$75 \mathrm{mM} \quad \mathrm{CaCl}_{2}$
$10 \mathrm{mM} \quad \mathrm{KCl}$
$15 \%(\mathrm{v} / \mathrm{v})$
$\mathrm{pH}$ adjusted to 7.0 with $\mathrm{KOH}$
sterile filtered and stored at $4{ }^{\circ} \mathrm{C}$




\subsection{PCR for cloning}

During cloning, different DNA polymerases were used depending on the individual characteristics of the template used. To generate high-quality PCR products (up to $5 \mathrm{~kb}$ ) from normal or GC-reach templates (e.g. BAC) Q5 High-Fidelity DNA Polymerase (NEB, cat\# M0491G) or Advantage ${ }^{\circledR}$ HD Polymerase Mix (Clontech, cat\# PT3921-2) were used. The AccuPrime $^{\mathrm{TM}}$ Pfx SuperMix (Invitrogen ${ }^{\mathrm{TM}}$, cat\# 12344-040) was used to generate highquality PCR product up to $8.5 \mathrm{~kb}$ from a GC-reach template. The reactions were set up as follows.

\section{Q5 High-Fidelity DNA Polymerase}

$\begin{array}{ll}5 \mu \mathrm{L} & \text { 5-fold Q5 reaction buffer } \\ 200 \mu \mathrm{M} & \text { dNTPs } \\ 0.5 \mu \mathrm{M} & \text { forward primer } \\ 0.5 \mu \mathrm{M} & \text { reverse primer } \\ \text { up to } 0.5 \mu \mathrm{g} & \text { template DNA } \\ 0.25 \mu \mathrm{L} & \text { Q5 polymerase } \\ \operatorname{ad} 25 \mu \mathrm{L} & \mathrm{ddH}_{2} \mathrm{O}\end{array}$

Advantage ${ }^{\circledR}$ HD Polymerase Mix

$5 \mu \mathrm{L} \quad 5$-fold HD buffer

$2 \mu \mathrm{L} \quad$ dNTP mixture (2.5 $\mathrm{mM}$ each)

$0.2-0.3 \mu \mathrm{M}$ forward primer

$0.2-0.3 \mu \mathrm{M}$ reverse primer

$200 \mathrm{ng} \quad$ template (50 ng for GC-reach)

$0.25 \mu \mathrm{L} \quad \mathrm{HD}$ polymerase

ad $25 \mu \mathrm{L} \quad \mathrm{ddH}_{2} \mathrm{O}$

\section{$\underline{\text { AccuPrime }^{\mathrm{TM}} \text { Pfx SuperMix }}$}

$\begin{array}{ll}22.5 \mu \mathrm{L} & \text { AccuPrime Pfx SuperMix } \\ 200 \mathrm{nM} & \text { forward primer } \\ 200 \mathrm{nM} & \text { reverse primer } \\ 10 \mathrm{pg}-200 \mathrm{ng} & \text { template DNA } \\ \operatorname{ad} 25 \mu \mathrm{L} & \mathrm{ddH}_{2} \mathrm{O}\end{array}$

thermocycling conditions

$\left.\begin{array}{ll}98^{\circ} \mathrm{C} & 30 \text { seconds } \\ 98^{\circ} \mathrm{C} & 5-10 \text { seconds } \\ 50-72^{\circ} \mathrm{C} & 10-30 \text { seconds } \\ 72^{\circ} \mathrm{C} & 20-30 \text { seconds } / \mathrm{kb}\end{array}\right]$ 25-35 cycles

$4-10^{\circ} \mathrm{C}$

thermocycling conditions

$\left.\begin{array}{ll}98^{\circ} \mathrm{C} & 5 \text { minutes } \\ 98^{\circ} \mathrm{C} & 10 \mathrm{sec} /(45 \mathrm{sec} \text { for GC-reach) } \\ 55^{\circ} \mathrm{C} & 5-15 \text { seconds } \\ 72^{\circ} \mathrm{C} & 1 \text { minute } / \mathrm{kb}\end{array}\right] 30$ cycles

$4-10^{\circ} \mathrm{C}$

thermocycling conditions

$\left.\begin{array}{ll}95^{\circ} \mathrm{C} & 5 \text { minutes } \\ 95^{\circ} \mathrm{C} & 15 \text { seconds } \\ 55-65^{\circ} \mathrm{C} & 30 \text { seconds } \\ 68^{\circ} \mathrm{C} & 1 \text { minute } / \mathrm{kb}\end{array}\right] 35$ cycles




\subsection{DNA digestion}

For digestion of plasmid and BAC DNA, restriction endonucleases with the supplied buffers were used. Generally, up to $3 \mu \mathrm{g}$ of plasmid DNA and up to $0.5 \mu \mathrm{g}$ of BAC DNA were digested in a 50 and $25 \mu \mathrm{L}$ reactions respectively, according to the manufacturer's instruction.

\subsection{Agarose gel electrophoresis (0.4 - $3 \%)$}

For agarose gel electrophoresis, DNA samples were mixed with 6-fold (v/v) DNA sample buffer and loaded on the agarose gel stained with Roti ${ }^{\circledR}-$ GelStain $(3.5 \mu \mathrm{L}$ for $50 \mathrm{~mL}$ gel; Carl Roth, cat\# 3865). The concentration of the agarose gel was adapted to the size of the analyzed DNA fragment: $3 \%$ agarose for fragment size $<500 \mathrm{bp}, 1 \%$ agarose for fragment sizes between 500 and 5,000 bp, and 0.5-0.4 \% agarose for fragment size > $5,000 \mathrm{bp}$ (e.g. BAC fragments). The electrophoresis was performed in TAE buffer till the desired DNA fragment separation. The DNA was visualized using ChemiDoc ${ }^{\mathrm{TM}}$ MP Imaging system.

$\underline{0.4-3.0 \% \text { agarose gel }}$

$1 \%(w / v)$ agarose in

$1 \times$ TAE buffer

$\underline{1 \times \text { TAE buffer }}$

$20 \mathrm{~mL} 50 \times$ TAE buffer

ad $1 \mathrm{LddH} \mathrm{H}_{2} \mathrm{O}$ $\underline{50 \times \text { TAE buffer }}$

$\begin{array}{ll}242 \mathrm{~g} & \text { TRIS-HCl } \\ 57.2 \mathrm{~mL} & \text { acetic acid } \\ 100 \mathrm{~mL} & 0.5 \mathrm{M} \text { EDTA, pH } 7.8 \\ \text { ad } 1 \mathrm{~L} & \mathrm{ddH}_{2} \mathrm{O}\end{array}$

$\underline{6 \times \text { DNA sample buffer }}$

$200 \mu \mathrm{L} \quad$ DNA gel loading dye (NEB, cat\# B7025)

$100 \mu \mathrm{L} \quad$ glycerol

ad $1 \mathrm{~mL} \quad \mathrm{dd} \mathrm{H}_{2} \mathrm{O}$

\subsection{DNA gel purification}

Digested backbone plasmids and PCR fragments were purified directly using the NucleoBond Gel and PCR Clean-up (Macherey Nagel, cat\# 740609), according to the manufac-

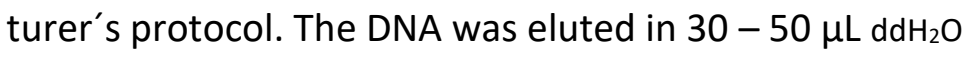




\subsection{DNA ligation}

The digested and on gel purified backbone plasmids were ligated with PCR-generated inserts following different ligation strategies: T4 ligase-mediated ligation, quick DNA ligation, DNA assembly, or in vivo DNA assembly. ${ }^{206}$ Before ligation, the digested backbone plasmid DNA was treated by the calf intestine phosphatase (CIP) to remove $3^{\prime}$ and $5^{\prime}$ phosphate residuals and therefore to prevent the possible re-ligation of the backbone vector. The reactions were set up as follows.

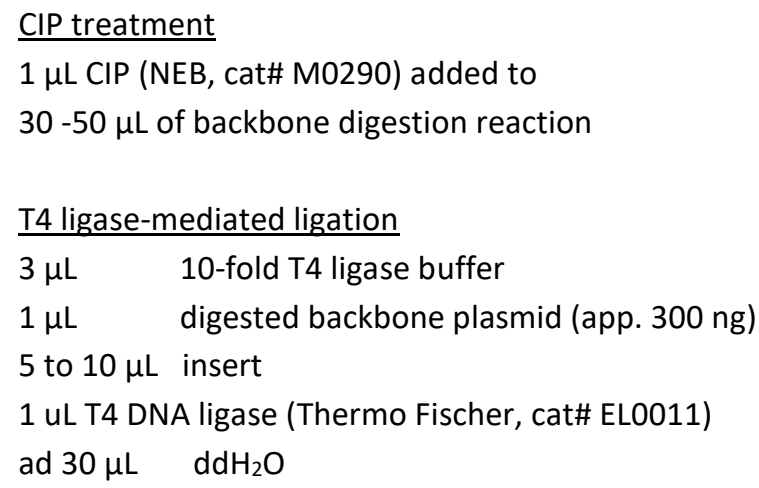

\subsection{Heat-transformation of competent E.coli}

For heat-transformation, chemo-competent E. coli DH5 $\alpha, \mathrm{DH} 10 \mathrm{~B}, \mathrm{SCS} 110$, and PIR1 were thawed on ice. Up to $20 \mu \mathrm{L}$ of ligation reaction were added to the thawed E. coli followed by gently mix via tube inverting and incubation on ice for $10 \mathrm{~min}$. Next, the bacteria were heated for 45 seconds to $42^{\circ} \mathrm{C}$, immediately followed by incubation for 5 minutes on ice. After $1 \mathrm{~mL}$ of $37^{\circ} \mathrm{C}$ warm lysogeny broth (LB) medium was added, the transformation reaction was shaken for 20 to 40 minutes at $37^{\circ} \mathrm{C}$. The bacteria were collected by centrifugation for $5 \mathrm{~min}$ at 3,000 $\times \mathrm{g}$ and streaked out on a LB agar plate containing the corresponding selection antibiotic (Table 2.14). It is crucial to use low salt LB (LB-Luria) and LB-Luria agar for bacterial clone selection with salt sensitive hygromycin antibiotic. The plate was incubated for 12 to 16 hours at $37^{\circ} \mathrm{C}$ and stored at $4^{\circ} \mathrm{C}$. 
Table 2.14: The used antibiotics, stock and working concentrations.

\begin{tabular}{llcl}
\hline antibiotic & $\begin{array}{l}\text { stock concentration } \\
(\mathrm{mg} / \mathrm{mL}), \text { diluent }\end{array}$ & $\begin{array}{c}\text { working concentration } \\
(\mu \mathrm{g} / \mathrm{mL})\end{array}$ & comment \\
\hline ampicillin & $50, \mathrm{dd} \mathrm{H}_{2} \mathrm{O}$ & 100 & $-20^{\circ} \mathrm{C}$ storage \\
tetracycline & 10, ethanol & 5 & light sensitive, $-20^{\circ} \mathrm{C}$ storage \\
chloramphenicol & 30, ethanol & 12.5 & $-20^{\circ} \mathrm{C}$ storage \\
kanamycin & $10, \quad \mathrm{ddH}_{2} \mathrm{O}$ & 30 & $-20^{\circ} \mathrm{C}$ storage \\
hygromycin & $100, \mathrm{dd} \mathrm{H}_{2} \mathrm{O}$ & 20 & low-salt LB, light sensitive, \\
& & & $4^{\circ} \mathrm{C}$ storage \\
\hline
\end{tabular}

\section{$\underline{\text { LB-Miller medium }}$}

$25 \mathrm{~g}$ LB powder

(10 g/L tryptone, $5 \mathrm{~g} / \mathrm{L}$ yeast extract, $10 \mathrm{~g} / \mathrm{L} \mathrm{NaCl} ; \mathrm{pH} 7.0$; Carl Roth, cat\# X968)

ad $1 \mathrm{LddH} \mathrm{H}_{2} \mathrm{O}$

autoclaved

$\underline{\text { LB-Luria medium }}$

15.5 g LS-LB powder

(10 g/L tryptone, $5 \mathrm{~g} / \mathrm{L}$ yeast extract, $0.5 \mathrm{~g} / \mathrm{L} \mathrm{NaCl} ; \mathrm{pH} 7.0$;

Sigma Aldrich, cat\# L3397)

ad $1 \mathrm{~L} \mathrm{dd} \mathrm{H}_{2} \mathrm{O}$

autoclaved $\underline{\text { LB-Miller agar }}$

40 g LB-agar powder

$(10 \mathrm{~g} / \mathrm{L}$ tryptone, $5 \mathrm{~g} / \mathrm{L}$ yeast extract, $10 \mathrm{~g} / \mathrm{L} \mathrm{NaCl}, 15 \mathrm{~g} / \mathrm{L}$ agaragar; pH 7.0; Carl Roth, cat\# X969)

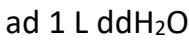

autoclaved

$\underline{\text { LB-Luria agar }}$

$15.5 \mathrm{~g}$ LS-LB powder

$(10 \mathrm{~g} / \mathrm{L}$ tryptone, $5 \mathrm{~g} / \mathrm{L}$ yeast extract, $0.5 \mathrm{~g} / \mathrm{L} \mathrm{NaCl} ; \mathrm{pH} 7.0$;

Sigma Aldrich, cat\# L3397)

$5 \%(w / v)$ agar-agar (Sigma Aldrich, cat\# A5306)

ad $1 \mathrm{LddH} \mathrm{H}_{2} \mathrm{O}$

autoclaved

\subsection{Plasmid preparation according to Holmes and Quigley}

Following heat-transformation (chapter 2.19), single clones grown on a selective agar plates were picked with sterile pipette tips, inoculated in $5 \mathrm{~mL}$ of LB medium containing the corresponding antibiotic (Table 2.14 ) and grown at $37^{\circ} \mathrm{C}$ overnight. To identify positive clones, DNA plasmid preparation was performed according to Holmes and Quigley. ${ }^{207}$ In short, $1.5 \mathrm{~mL}$ of overnight culture was collected and centrifuged for $1 \mathrm{~min}$ at $16,200 \times \mathrm{g}$ at room temperature. After supernatant was removed, the bacteria pellet was resuspended in $400 \mu \mathrm{L}$ of $4^{\circ} \mathrm{C}$ STET buffer followed by addition of $25 \mu \mathrm{L}$ of lysozyme, gently mix, and incubation for $2 \mathrm{~min}$ at room temperature. Next, bacteria were boiled for 30 seconds at $95^{\circ} \mathrm{C}$ and the broken cells were centrifuged for $10 \mathrm{~min}$ at $16,200 \times \mathrm{g}$ at room temperature. After centrifugation, cell debris were removed using a sterile toothpick and the supernatant was supplemented with $40 \mu \mathrm{L}$ of $3 \mathrm{M}$ sodium acetate $\mathrm{pH} 5.2$ 
and $420 \mu \mathrm{L}$ of isopropanol. The plasmid DNA-containing precipitate was pelletized by centrifugation for $15 \mathrm{~min}$ at $16,200 \times \mathrm{g}$ at room temperature. Next, supernatant was removed and DNA-containing pellet washed with $1 \mathrm{~mL}$ of $70 \%(\mathrm{v} / \mathrm{v})$ ethanol, followed by centrifugation for $10 \mathrm{~min}$ at $16,200 \times \mathrm{g}$ at room temperature. Finally, after ethanol supernatant was removed, DNA-containing pellet was air-dried and resuspended in 30 $\mu \mathrm{L}$ double distilled water.

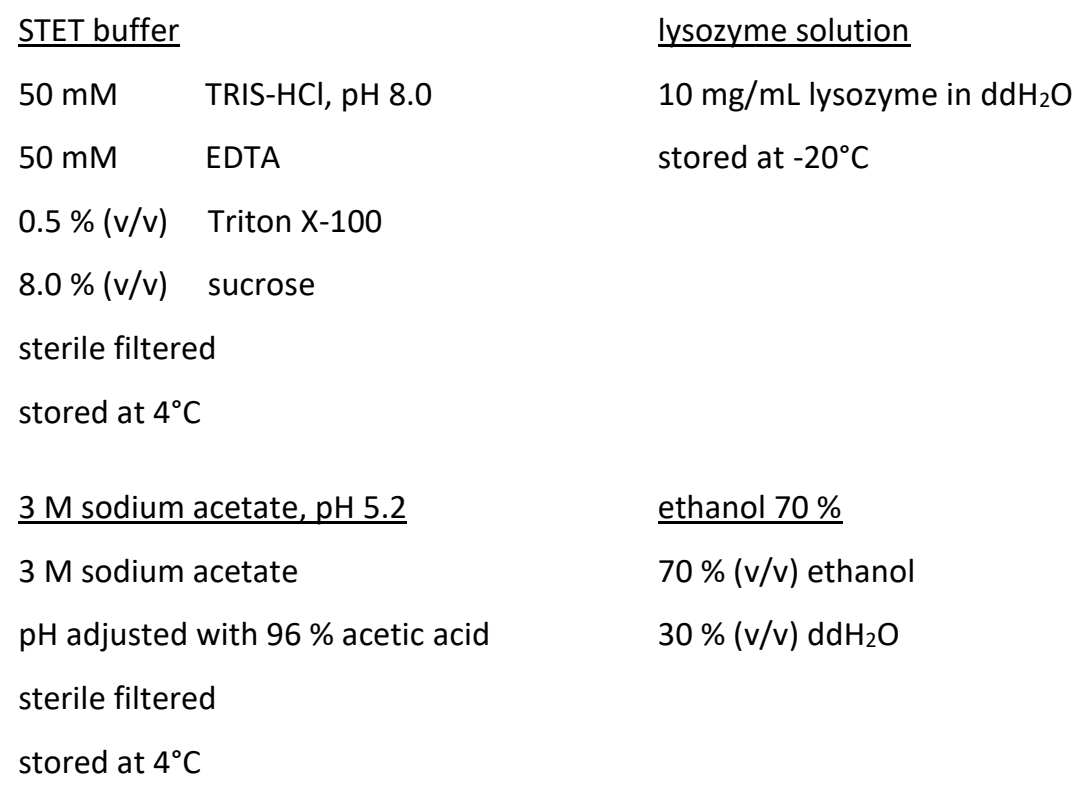

\subsection{Selection of positive clones}

Depending on cloning strategy, bacterial colonies grown on selective agar plates were tested through restriction digestion or colony PCR by Red Taq DNA polymerase (VWR, cat\# 733-1320). The oligonucleotides used for colony PCR are summarized in Table 2.15. Restriction digestion was performed following plasmid DNA isolation via Holmes and Quigley strategy (chapter 2.20). The reactions were set up as follows.

\section{Red Taq DNA Polymerase Master Mix}

$12.5 \mu \mathrm{L}$ Taq 2-fold master mix

$200 \mathrm{nM}$ forward primer

$200 \mathrm{nM}$ reverse primer

1-3 $\mu \mathrm{L}$ of picked colony in $\mathrm{dd}_{2} \mathrm{O}$

ad $25 \mu \mathrm{L} \quad \mathrm{ddd}_{2} \mathrm{O}$ test restriction digestion

$\begin{array}{ll}2.5 \mu \mathrm{L} & \text { 10-fold reaction buffer } \\ 10-15 \mu \mathrm{L} & \text { plasmid DNA } \\ 0.3 \mu \mathrm{L} & \text { of each restriction enzyme } \\ 1 \mu \mathrm{L} & \text { RNAse A (VWR, cat\# AC117) } \\ \operatorname{ad} 25 \mu \mathrm{L} & \mathrm{ddH}_{2} \mathrm{O}\end{array}$


thermocycling conditions

$95^{\circ} \mathrm{C} \quad 2$ minutes

$95^{\circ} \mathrm{C} \quad 30$ seconds

$52-55^{\circ} \mathrm{C} \quad 25$ seconds

30 cycles

$72^{\circ} \mathrm{C} \quad 1$ minute $/ \mathrm{kb}$

$72^{\circ} \mathrm{C} \quad 2$ minutes

$4-10^{\circ} \mathrm{C}$ hold

Table 2.15: Sequences of oligonucleotides used for colony PCR.

\begin{tabular}{lll}
\hline vector & oligonucleotide & sequence in 5' to 3' direction \\
\hline pcDNA3.1(-)-CMV-GLuc-myc & F: PGK_bGH & $\begin{array}{l}\text { AAAGCGCCTCCCCTACCCGGTAGCCA- } \\
\text { TAGAGCCCACCGCATCCCCAGCAT }\end{array}$ \\
\cline { 2 - 3 } & R: EcoRV_CMV & TCTGCAGATATCGACATTGATTATTGA \\
\hline pcDNA3.1(-)-H2G-NLS-CMV-GLuc-myc & F: Xho_pA_Gluc & TCTTACTCGAGGAAGGGTTCCGCAAGCT \\
\cline { 2 - 3 } & R: bGH_PGK & $\begin{array}{l}\text { GGGATGCGGTGGGCTCTATGGCTAC- } \\
\text { CGGGTAGGGGAGGCGCTTTTCCCAA }\end{array}$ \\
\hline $\begin{array}{ll}\text { PcDNA3.1(-)-Prp-SEAP-myc-NLS-H2G-NLS- } \\
\text { pR6k-Prp-SEAP-myc-NLS-H2G-NLS-CMV- } \\
\text { GLuc-myc }\end{array}$ & F: Nhe_Prp_SEAP & CTGGCTAGCATGGCGAACCTTAGCTA \\
\cline { 2 - 3 } & R: Xho_SV40 & CCTTCCTCGAGTAAGATACATTGATGA \\
$\begin{array}{l}\text { pcDNA3.1(-)-CEBPD-Prp-SEAP-myc-NLS- } \\
\text { H2G-NLS-CMV-GLuc-myc }\end{array}$ & F: PCR_CEBPD_cassette & $\begin{array}{l}\text { TATTGGAGTGTCATTCCCAGCAGCG- } \\
\text { CAGCGC }\end{array}$ \\
\hline $\begin{array}{l}\text { pcDNA3.1(-)-CEBPD-Prp-SEAP-myc-NLS- } \\
\text { H2G-NLS-CMV-GLuc-myc }\end{array}$ & R: qPCR_SEAP_WT & CGCCCAGGAAGATGATGAGG \\
\hline
\end{tabular}

\subsection{Plasmid preparation using kits}

For subsequent procedures such as DNA sequencing and cell transfection, plasmid DNA was isolated using NucleoBond Plasmid mini and midi preparation kits (Macherey \& Nagel, cat\# 740571, cat\# 740573). For isolation of high-quality BAC DNA NucleoBond Xtra BAC preparation kit (Macherey \& Nagel, cat\# 740436) was used.

\subsection{DNA sequencing}

Sequencing of plasmid DNA was performed by company Microsynth AG, Balgach, Switzerland. BAC DNA was sequenced by Zeda Sequencing Service, Fraunhofer IME, Aachen. 


\subsection{Glycerol stock generation}

After identification of a positive bacterial cell clone, a permanent glycerol stock with 800 $\mu \mathrm{L}$ of fresh overnight culture and $200 \mu \mathrm{L}$ of sterile $86 \%$ (v/v) glycerol was generated. Glycerol stocks were stored at $-80^{\circ} \mathrm{C}$.

\subsection{Bacterial artificial chromosome cloning}

\subsubsection{Verification of a wild type BAC clone}

To isolate high quality BAC DNA, DH10B bacteria containing BAC clone CH17-293N3 (201,427 bp; BACPAC CHORI, Table 2.12) was grown in LB-Miller medium supplemented with $12.5 \mu \mathrm{g} / \mathrm{mL}$ chloramphenicol for $12-14$ hours at $37^{\circ} \mathrm{C}$ and $220 \mathrm{rpm}$, till $\mathrm{OD}_{600}$ with 0.35 - 0.4. Next, BAC DNA was isolated (chapter 2.22) and verified by restriction digestion, PCR (for primers refer to Table 2.16), and sequencing (for primers refer to Table 2.11). The restriction digestion, $P C R$, and sequencing reactions were set up as follows.

BAC restriction digestion

$2.5 \mu \mathrm{L} \quad$ 10-fold CutSmart buffer

$0.5 \mu \mathrm{L} \quad$ BamHI restriction enzyme

500 ng BACDNA

ad $25 \mu \mathrm{LddH} \mathrm{H}_{2} \mathrm{O}$

incubated for 2 hours at $37^{\circ} \mathrm{C}$

electrophoresis on 0.4 \% TAE agarose gel (chapter 2.16),

for 1.5 hours at $3 \mathrm{~V} / \mathrm{cm}$

BAC sequencing

900 ng BAC DNA per primer reaction

$10 \mu \mathrm{M}$ primer

ad $15 \mu \mathrm{LddH} \mathrm{H}_{2} \mathrm{O}$
BAC verification PCR

$5 \mu \mathrm{L} \quad 5$-fold HD buffer

$0.5 \mu \mathrm{L} \quad \mathrm{dNTP}$ mixture (10 $\mathrm{mM}$ each)

$200 \mathrm{mM}$ forward primer

$200 \mathrm{mM}$ reverse primer

$5 \mathrm{ng} \quad$ BAC DNA template

$0.25 \mu \mathrm{L} \quad$ Advantage $^{\circledR} \mathrm{HD}$ Polymerase

ad $25 \mu \mathrm{L} \quad \mathrm{ddH}_{2} \mathrm{O}$

for PCR program see chapter 2.14

Table 2.16: Primer pairs used for BAC clone verification by PCR.

\begin{tabular}{llcl}
\hline template & primer pairs (5'to 3' orientation) & product size (bp) & verified BAC region \\
\hline & forward: CCT CCA GCA GCC TCT ACA GT & 669 & 3' UTR and down- \\
& reverse: TCT TGG GAC ATA GGA GCG CA & & stream sequence \\
\hline & forward: CAG CAA CGA CCC ATA CCT CA & \multirow{2}{*}{$\begin{array}{c}\text { CEBPD mRNA } \\
\text { reverse: TCT TTG CGC TCC TAT GTC CC }\end{array}$} & \\
\hline
\end{tabular}




\begin{tabular}{lll}
\hline forward: TCT TTG CGC TCC TAT GTC CC & 353 & $\begin{array}{l}\text { CEBPD protein and } \\
\text { 3' UTR }\end{array}$ \\
reverse: AGC GCA ACA ACA TCG CC & 272 & CEBPD protein \\
\hline forward: AAG CTC ACC ACG GTC TGT & & \\
reverse: ATC GAC TTC AGC GCC TAC AT & 1946 & upstream sequence \\
\hline forward: GTT TCC CTT CTG CCT TCG GG & &
\end{tabular}

UTR: untranslated region

\subsubsection{BAC in vivo recombination (preliminary protocol)}

BAC in vivo recombination relies on a homologous recombination between a linear (e.g. PCR product) and a circular DNA molecule (e.g. BAC) allowing flexible, precise, and accurate DNA modification. ${ }^{208}$ Homological recombination is mediated by recombinase proteins (e.g. rec- $\alpha$, rec- $\beta$, and rec- $\gamma$ ) and requires homology regions - pieces of DNA shared by the two molecules that recombine. As homology region sequences can be chosen freely, any position on a target molecule can be specifically altered. ${ }^{208}$

In this study, first experiments were done to generate recombinant BAC vector that should encode multi-gene-reporter cassette 2.0 under control of CEBPD target promoter. The multi-gene-reporter cassette 2.0 was constructed in a high-copy pcDNA3.1(-) backbone (chapter 8.8.4, Fig.8-40) that is easy to handle, and then cloned into pR6k backbone (chapter 8.8.5, Fig.8-41) that can be replicated exclusively in PIR1 E.coli (Table 2.8). The use of pR6k backbone eliminates a possibility of its replication in DH10B E.coli during recombination.

In short, CH17-293N3 BAC-expressing DH10B E.coli were heat-transformed (chapter 2.19) with pSC101-gbaA vector (Fig.8-26) that encodes rec- $\alpha$, rec- $\beta$, and rec- $\gamma$ recombinase proteins under control of L-arabinose-inducible promoter and contains a thermosensitive origin of replication (replication abolished at $37^{\circ} \mathrm{C}$ ). The insert, which encodes multi-gene-reporter cassette 2.0 flanked by 50 bp long homology regions, was generated by PCR (AccuPrime ${ }^{\mathrm{TM}}$ Pfx SuperMix, chapter 2.14) using pR6k-Prp-SEAP-myc-NLSH2G-NLS-CMV-GLuc-myc vector (Fig.8-41) as a template. The pSC101-gbaA-containing, BAC-expressing DH10B E.coli were cultured in $1.5 \mathrm{~mL}$ LB-Luria medium supplemented with $12.5 \mu \mathrm{g} / \mathrm{mL}$ chloramphenicol (ca) and $5 \mu \mathrm{g} / \mathrm{mL}$ tetracycline (tet) at $30^{\circ} \mathrm{C}$ and 1000 
rpm, overnight. Next day, $50 \mu \mathrm{L}$ of the overnight culture were inoculated in $1.4 \mathrm{~mL}$ of fresh ca + tet conditioned LB-Luria medium and incubated for 2 hours at $30^{\circ} \mathrm{C}$ and 1000 rpm till $\mathrm{OD}_{600} \sim 0.3$. Next, $50 \mu \mathrm{L}$ of $10 \%(\mathrm{w} / \mathrm{v})$ L-arabinose, which induces recombinase protein expression, were added to the bacterial suspension, followed by incubation for 1 hour at $37^{\circ} \mathrm{C}$ and $1000 \mathrm{rpm}$.

\subsubsection{Bacteria electroporation and positive clone selection (preliminary protocol)}

After L-arabinose treatment, bacterial cells were collected by centrifugation for 2 minutes $3,000 \times \mathrm{g}$ and $2^{\circ} \mathrm{C}$. Next, supernatant was removed and bacterial pellet was resuspended in $1 \mathrm{~mL}$ of ice-cold cell culture grade deionized water. Centrifugation and on ice resuspension steps were repeated. A small volume 1-2 $\mu \mathrm{L}$ of fresh PCR product (insert, $0.2-1 \mu \mathrm{g}$ ) was added to up to $30 \mu \mathrm{L}$ of bacterial suspension, gently mixed, placed into an ice-cold electroporation cuvette (Bio-Rad, $0.1 \mathrm{~cm}$ gap, cat\# 1652083), and electroporated at $1350 \mathrm{~V} 10 \mu \mathrm{F}$ and 600 Ohms for optimally $5 \mathrm{~ms}$ using Gene Pulser Xcell ${ }^{\mathrm{TM}}$ electroporation system (Bio-Rad). Immediately after electroporation, bacteria were collected in $37^{\circ} \mathrm{C}$ warm SOC medium without antibiotics and incubated for 70 minutes at $37^{\circ} \mathrm{C}$ and $1000 \mathrm{rpm}$. Finally, bacteria were streaked out on LB-Luria agar plates conditioned with $12.5 \mu \mathrm{g} / \mathrm{mL}$ chloramphenicol $+20 \mu \mathrm{g} / \mathrm{mL}$ hygromycin and incubated at $37^{\circ} \mathrm{C}$, overnight. Grown cell colonies were tested using UV-light to detect copGFP fluorescence and by colony PCR (chapter 2.21).

$\underline{\text { SOC medium }}$

$1 \mathrm{~L} \quad$ LB-Luria medium (chapter 2.19)

autoclaved

$20 \mathrm{mM}$ glucose

sterile filtered $(0.2 \mu \mathrm{m}$ filter $)$
$10 \%(w / v)$ L-arabinose stock solution

$0.5 \mathrm{~g} \mathrm{L(+)-arabinose} \mathrm{(Carl} \mathrm{Roth,} \mathrm{cat \#} \mathrm{5118)}$ ad $5 \mathrm{~mL}$ dd $\mathrm{H}_{2} \mathrm{O}$

\subsection{Eukaryotic cell culture}

HEK293T wild type and reporter cells were cultured in DMEM GlutaMAX ${ }^{\mathrm{TM}}$ (Thermo Fisher Scientific, cat\# 31966047) supplemented with $10 \%$ (v/v) heat-inactivated FBS (Thermo Fisher Scientific, cat\# 10270-106), 1 \% (v/v) pen-strep $(10,000 \mathrm{U} / \mathrm{mL}$, Thermo 
Fisher Scientific, cat\# 15140-122). Stably transfected HEK293T reporter cells were cultured in cell culture medium additionally supplemented with $10 \mu \mathrm{g} / \mathrm{mL}$ puromycin dihydrochlorid (Carl Roth, cat\# 0240) for selective pressure.

THP-1 wild type and reporter cells were cultured in RPMI 1640 GlutaMAX ${ }^{\mathrm{TM}}$ medium (Thermo Fisher Scientific, cat\# 61870-044) supplemented with 10 \% (v/v) heat-inactivated FBS, $1 \%(\mathrm{v} / \mathrm{v})$ penicillin-streptomycin solution. During screening, THP-1 reporter cells were cultured in RPMI 1640 medium without phenol red (Thermo Fisher Scientific, cat\# 11835105), supplemented with $10 \%$ (v/v) heat-inactivated FBS, $1 \%$ (v/v) pen-strep, $2 \mathrm{mM}$ glutamine (Thermo Fisher Scientific, cat\# 25030081). All cells were incubated at $37^{\circ} \mathrm{C}$, in an atmosphere of $5 \% \mathrm{CO}_{2}$ and $95 \%$ humidity. Generated HEK293T and THP-1 reporter cells were tested for mycoplasma contamination using mycoplasma detection kit (Lonza, cat\# LT07-318) after cell sorting.

For propagation, HEK293T cells were washed with Dulbecco's phosphate-buffered saline, DPBS (no calcium, no magnesium, pH 7.0-7.3, Thermo Fisher Scientific, cat\# 14190169) and detached with trypsin-EDTA (Thermo Fischer Scientific, cat\# 25300054) solution. Trypsin-treated cells were resuspended in DMEM GlutaMAX ${ }^{\mathrm{TM}}$ medium and propagated in a ratio of 1:10 to 1:15. THP-1 suspension cells were collected by centrifugation for 5 min at $300 \times \mathrm{g}$, resuspended in RPMI 1640 GlutaMAX $^{\mathrm{TM}}$ medium and propagated in a ratio of $1: 10$ to $1: 15$.

For experiments, HEK293T and THP-1 reporter cells were seed in different multi-well formats. The routinely used cell numbers are listed in Table 2.17.

Table 2.17: Routinely used cell numbers for culture in multi-well format.

\begin{tabular}{lcc}
\hline multi-well plate format & HEK293T (cells/well) & THP-1 / THP-1 M $\boldsymbol{\varphi}$ (cells/well) \\
\hline 6-well format & 600,000 & 650,000 \\
24-well format & - & 120,00 \\
96-well format & - & 40,000 \\
\hline
\end{tabular}

\subsection{Freezing and thawing}

\section{Freezing}

For long-term storage, cells were frozen in liquid nitrogen. HEK293T cells, when reached 
around $80 \%$ confluence in a $175 \mathrm{~cm}^{2}$ flask, were washed with $15 \mathrm{~mL}$ DPBS, detached with $10 \mathrm{~mL}$ trypsin-EDTA and collected in $30 \mathrm{~mL}$ DMEM GlutaMAX ${ }^{\mathrm{TM}}$ medium followed by a centrifugation for $5 \mathrm{~min}$ at $300 \times \mathrm{g}$. THP-1 suspension cells, when reached cell concentration with 800,000 - 900,000 cells per milliliter, were directly collected by centrifugation for $5 \mathrm{~min}$ at $300 \times \mathrm{g}$. The cellular supernatant was collected for mycoplasma test. The cell pellet was resuspended in $10 \%(\mathrm{v} / \mathrm{v})$ DMSO/FBS with up to 5 million cells per millilitre, split on corresponding number of cryo-tubes, and frozen in freezing container at $-80^{\circ} \mathrm{C}$. Frozen cells were finally transferred to the liquid nitrogen tank upon negative mycoplasma test result.

\section{$10 \%$ DMSO / FBS}

$10 \%$ DMSO (v/v)

ad final volume FBS

\section{Thawing}

Frozen cells were thawed through the short incubation of cell-containing cryo-tube at $37^{\circ} \mathrm{C}$ in water bath and subsequently transferred to a $14 \mathrm{~mL}$ of $37^{\circ} \mathrm{C}$ warm cell culture medium in a $15 \mathrm{~mL}$ tube, followed by centrifugation for $5 \mathrm{~min}$ at $300 \times \mathrm{g}$. The cell-pellet was resuspended in corresponding cell culture medium and transferred to a culture flask. To remove residual DMSO, the cell culture medium was changed 24 hours after thawing.

\subsection{Generation of reporter cells}

\subsubsection{Transient and stabile transfection of HEK293T cells}

\section{Transient transfection}

Wild type HEK293T cells were transfected with Lipofectamine ${ }^{\circledR} 2000$ transfection reagent (Invitrogen ${ }^{\mathrm{TM}}$, Thermo Fischer Scientific, cat\# 11668027) one day after cell seed in a 6-well format (Table 2.17), according to the manufacturer's protocol. In short, plasmid DNA and Lipofectamine ${ }^{\circledR} 2000$ were first diluted in an appropriated volume of Opti$\mathrm{MEM}^{\circledR}$ reduced serum medium (Gibco ${ }^{\mathrm{TM}}$, Thermo Fischer Scientific, cat\# 31985062). Next, the diluted DNA was added to the diluted Lipofectamine ${ }^{\circledR} 2000(1: 1, v / v)$ and in- 
cubated for $5 \mathrm{~min}$ at room temperature. The DNA-Lipofectamine complex was added to the cells, followed by the incubation at $37^{\circ} \mathrm{C}$ for at least 12 hours.

\section{Stable transfection}

HEK293T reporter cells were generated by stably transfection of wild type HEK293T cells using Lipofectamine ${ }^{\circledR} 2000$ reagent in a 6-well format (see transient transfection). For cloning strategies of backbone and enzyme-encoding plasmids refer to chapter 8.8.1.

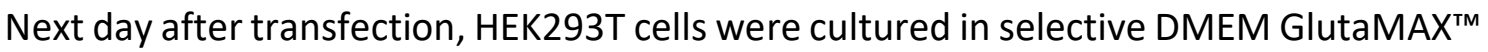
medium containing $10 \mu \mathrm{g} / \mathrm{mL}$ puromycin. Positive HEK293T cell clones, stably expressing generated eGFP-positive constructs, were raised up as single clones and selected via fluorescence activated cell sorting (FACS) approximately three weeks after transfection. Transfection reactions were set up as follows.

$\begin{array}{lll}\underline{\text { transient }} & \underline{\text { stable }} & \\ 2 \mu \mathrm{g} & 2.5 \mu \mathrm{g} & \text { DNA amount per well } \\ \text { ad } 125 \mu \mathrm{L} & \text { ad } 125 \mu \mathrm{L} & \text { Opti-MEM medium volume for DNA dilution } \\ 9 \mu \mathrm{L} & 9 \mu \mathrm{L} & \text { Lipofectamine } 2000 \text { per well } \\ \text { ad } 125 \mu \mathrm{L} & \text { ad } 125 \mu \mathrm{L} & \text { Opti-MEM medium volume for Lipofectamine dilution } \\ 250 \mu \mathrm{L} & 250 \mu \mathrm{L} & \text { total amount of DNA-Lipofectamine complex per well }\end{array}$

\subsubsection{Generation of THP-1 reporter cell lines by viral transduction}

THP-1 reporter cells stably expressing the multi-gene-reporter cassette 1.0 were generated via viral transduction. Cloning of corresponding vectors is described in chapter 8.8.3.

\section{Generation of viral particles}

Viral particles containing generated vectors (Fig.8-37, Fig.8-38) were produced using HEK293T wild type cells, which were transfected with jetPRIME ${ }^{\circledR}$ transfection reagent (Polyplus transfection ${ }^{\circledR}$, cat\# 114-07) in $10 \mathrm{~cm}^{2}$ dishes with 2 million cells per dish. One day after transfection, cellular supernatants were collected and centrifuged for 5 minutes at $500 \times \mathrm{g}$. To obtain highly concentrated viral particles, virus-containing supernatants were sterile-filtered and precipitated using PEG virus precipitation kit (Abcam, cat\# 102538) according to the manufacturer's protocol. In short, virus concentrating so- 
lution was added to supernatants and incubated for 24 hours at $4^{\circ} \mathrm{C}$, followed by centrifugation for 30 minutes at $3,200 \times \mathrm{g}$ and $4^{\circ} \mathrm{C}$. The virus-enriched phase was aliquoted and stored at $-80^{\circ} \mathrm{C}$. Transfection reaction was set up as follows.

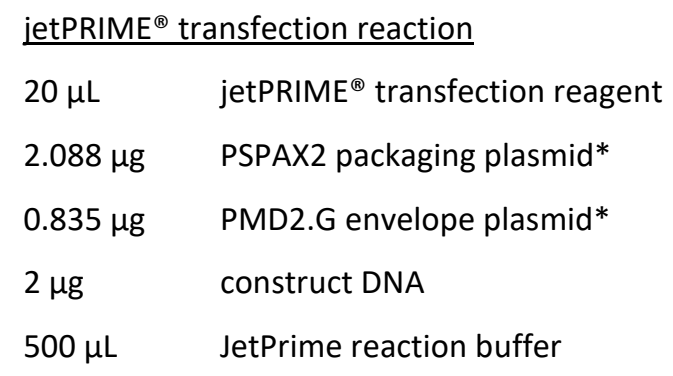

*Plasmids were kindly provided by the working group of Prof. Brüne, Institute of Biochemistry I - Pathobiochemistry, Goethe University, Frankfurt am Main

\section{Cell transduction}

Fresh aliquot of a virus-containing suspension was added to 1 million of wild type THP1 cells, which were cultured in $1.5 \mathrm{~mL}$ of medium in a 6-well format, and incubated for 6 hours. Next, $1 \mathrm{~mL}$ of fresh medium was added to the cells followed by cell culture for 24 hours. Virally transduced cells were expanded for up to four weeks, before positive eGFP-expressing THP-1 cell clones were selected by FACS.

\subsubsection{Generation of THP-1 reporter cells by electroporation (preliminary protocol)}

THP-1 reporter cells that were proposed to express the multi-gene-reporter cassette 2.0 were electroporated with the corresponding vector (Fig.8-42). For cloning strategy refer to chapter 8.8.6.

THP-1 cells were electroporated using Amaxa 4D-Nucleofector (Lonza) and the corresponding cell electroporation kit (V4XC-3024), according to the manufacturer's protocol. In short, wild type THP-1 cells were seeded in a 6-well format with 200,000 cells per well and cultured for one day, till 300-400,000 cells per well. Further, cells were combined to aliquots, each with two million cells, and centrifuged for 10 minutes at $90 \times \mathrm{g}$, and room temperature. After cell culture supernatants were removed, cells of each aliquot were resplendent in $100 \mu \mathrm{L}$ of a prepared Nucleofector solution, supplemented with $0.8-6 \mu \mathrm{g}$ of plasmid DNA (generated vector), and placed into electroporation cuvettes avoiding air bubbles. Placed into Nucleofector, cells were electroporated using 
different, from a supplier suggested electroporation programs. Just after electroporation, cells were resplendent in $0.5 \mathrm{~mL}$ of pre-warmed cell culture medium, transferred to a 6-well plate, and incubated as usual. Growing electroporated cells were continuously expanded before being sorted via FACS. Positive copGFP-expressing THP-1 reporter cells were cultured in a 6-well format after cell sorting. To the end of this timely restricted project, electroporated THP-1 reporter cells did not survive a FACS procedure due to very low transfection efficiency.

\subsection{SDS-PAGE and Western Blotting analysis}

\subsubsection{Preparation of cell extracts}

For Western Blotting analysis, wild type and stable transfected HEK293T cells were rinsed with DPBS and lysed in with protease inhibitor (cOmplete ${ }^{\mathrm{TM}}$ Protease Inhibitor Cocktail, Sigma-Aldrich, cat\# 11697498001) supplemented RIPA buffer, for 30 min on ice. For these experiment, HEK293T cells were used that remained in the well after immunofluorescence analysis (chapter 2.30). Cell lysates were cleared by centrifugation for $5 \mathrm{~min}$ at $10,000 \times \mathrm{g}, 4^{\circ} \mathrm{C}$ and stored at $-20^{\circ} \mathrm{C}$.

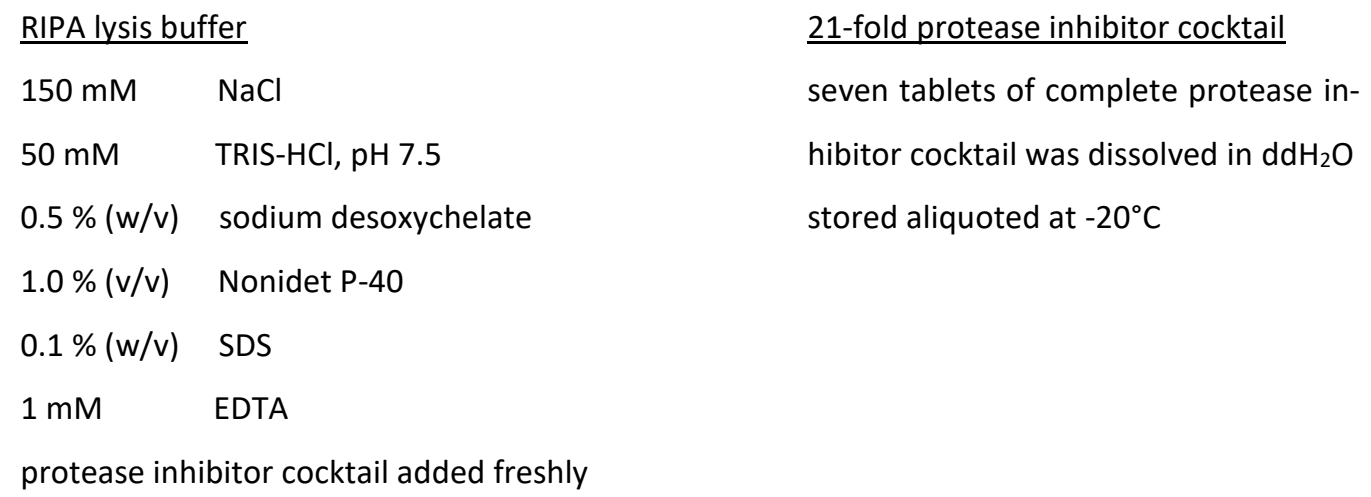

\subsubsection{Determination of protein concentration}

Protein concentration was determined using Pierce ${ }^{\mathrm{TM}}$ BCA Protein Assays Kit (Pierce, cat\# 23225) according to the manufacturer's protocol. The protein calibration curve was generated using supplied protein standards. Sample absorption, measured at $540 \mathrm{~nm}$ using EnSpire plate reader, was converted into the sample protein concentration using the generated protein calibration curve. Protein standards and samples were measured in RIPA lysis buffer. 


\subsubsection{SDS-polyacrylamide gel electrophoresis}

Protein samples were ssupplemented with 4-fold Laemmli buffer (Bio-Rad, cat\# 1610747) and boiled for $5 \mathrm{~min}$ at $95^{\circ} \mathrm{C}$. The 5 and $10 \mu \mathrm{g}$ of sample protein as well as protein marker (Bio-Rad, Fig.2-2) were loaded on the Mini-PROTEAN ${ }^{\circledR}$ TGX Stain-Free $^{\mathrm{TM}}$ Precast gel (8-16\%, Bio-Rad, cat\# 4568104) and separated for up to 1 hour at 85 mA.

\subsubsection{Protein blotting}

Proteins were transferred from gel to PVDF membrane $(0.45 \mu \mathrm{m}$, Millipore, cat\# IPVH00010) by a semi-dry blotting procedure using Trans-Blot ${ }^{\circledR}$ Turbo $^{\text {TM }}$ System (BioRad, 7 minutes blotting program). The membrane was first activated by incubation in $100 \%$ methanol for 5 minutes and additionally incubated with thick blot filter paper (Bio-Rad, cat\# 1703932) in the transfer buffer. After blotting, the membrane was incubated either in $5 \%(\mathrm{w} / \mathrm{v})$ BSA in PBS or in a blotting-Grade Blocker (Bio-Rad, cat \# 1706404), at $4^{\circ} \mathrm{C}$ overnight.

$\underline{1 \times \text { transfer buffer }}$

$200 \mathrm{~mL} \quad 5 \times$ transfer buffer (Bio-Rad)

$200 \mathrm{~mL}$ ethanol

ad $1 \mathrm{~L} \quad \mathrm{ddH}_{2} \mathrm{O}$

\subsubsection{Immunodetection of proteins}

Western Blotting membranes were incubated with primary (Table 2.6) and the HRP-conjugated secondary (Table 2.7) antibodies in PBST for $2 \mathrm{~h}$ and $1 \mathrm{~h}$ at room temperature, respectively. During antibody application membranes were washed three times with PBST for 5 min and three times shortly. After final rinse with PBS, membranes were developed using Clarity ${ }^{\mathrm{TM}}$ Western ECL Blotting Substrate (Bio-Rad, cat\# 1705060).

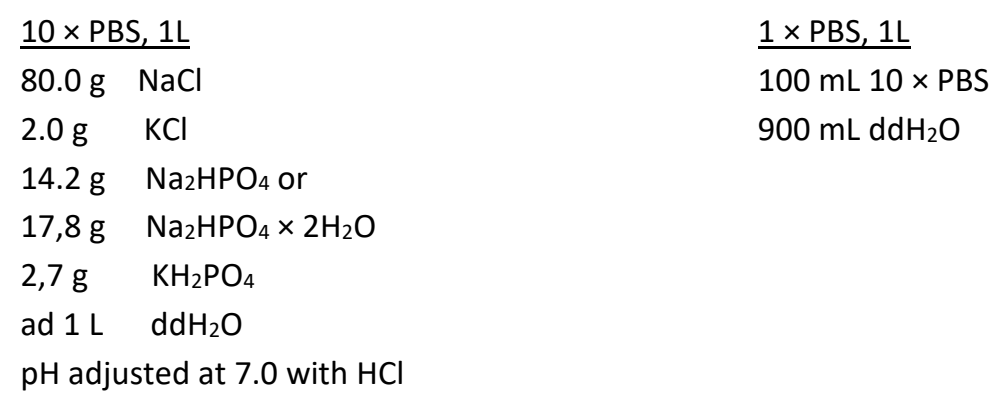


$\underline{\text { PBST, } 1 \mathrm{~L}}$

$1 \mathrm{~L} 1 \times$ PBS

$0.5 \mathrm{~mL}$ tween-20
BSA blocking solution

$5 \%(w / v)$ BSA in TBST

\subsection{Preparation of immunofluorescence samples}

For immunofluorescence analysis, generated HEK293T reporter cells were cultured on glass cover slips in a 6 -well format with 650,000 cells per well for $24 \mathrm{~h}$. Next day, after the cell culture medium was removed (used for SEAP enzymatic assay, chapter 2.34.1), cells were rinsed twice with the sterile DPBS and transferred on glass slips into a fresh plate. Here, cells were first fixed with $4 \%(\mathrm{v} / \mathrm{v})$ PFA in PBS for $20 \mathrm{~min}$, permeabilized with $0.1 \%(\mathrm{v} / \mathrm{v})$ Triton X-100 in PBS for 5 min and blocked with $10 \%(\mathrm{v} / \mathrm{v})$ FBS in PBS for $30 \mathrm{~min}$ at room temperature. Between the steps, cells were rinsed with PBS. Both, the primary anti-c-myc (1:100 in 10 \% (v/v) FBS in PBS) and Alexa Fluor 555 (1:1,000 in $10 \%$ (v/v) FBS in PBS) secondary antibodies were applied for 1.5 hours at room temperature. During antibody application cells were washed three times with PBS for 5 min. Nuclei were stained with DAPI for $15 \mathrm{~min}$ at room temperature. Through the procedure, reporter cells were protected from light. The DAPI-stained cells were mounted on glass slides using mowiol mounting solution and stored in the dark for long term at $4^{\circ} \mathrm{C}$.

$0.1 \%$ Triton $\mathrm{X}-100$

$0.1 \%$ Triton $X-100(v / v)$ in PBS

sterile filtered, stored at $4^{\circ} \mathrm{C}$

DAPI stock

$0.5 \mathrm{mg} / \mathrm{mL}$ DAPI in $\mathrm{dd}_{2} \mathrm{O}$

stored at $-20^{\circ} \mathrm{C}$

Mowiol mounting solution

$6 \mathrm{~g}$ glycerol

$2.4 \mathrm{~g}$ Mowiol 4-88

$12 \mathrm{~mL} \quad 0.2 \mathrm{M}$ TRIS-HCl, $\mathrm{pH} 8.5$

$6 \mathrm{~mL} \quad \mathrm{ddH}_{2} \mathrm{O}$

$450 \mathrm{mg}$ DABCO

stored aliquoted at $-20^{\circ} \mathrm{C}$
$10 \%$ FBS in PBS

$10 \%$ FBS $(v / v)$ in PBS

prepared freshly

$4 \%$ PFA

$4 \%$ paraformaldehyd $(w / v)$ in PBS

stored at $4^{\circ} \mathrm{C}$ 


\subsection{Cell microscopy}

\subsubsection{Live-cell microscopy}

Wild type and reporter THP-1 cells were examined via live-cell microscopy. Phase contrast and fluorescent pictures of cultured THP-1 cells were taken by Observer Z1 with AxioCam MRC camera or ZOE Fluorescent Cell Imager (Bio-Rad), respectively.

\subsubsection{Confocal fluorescent microscopy}

During immunofluorescence analysis, fixed HEK293T reporter cells were examined using Zeiss confocal microscopy and images were captured using Software (AxioVison v4.8).

\subsection{RNA purification and real-time quantitative PCR}

Total RNA was extracted using RNeasy Mini Kit (Qiagen, cat\# 74104), according to the manufacturer's instructions, including on-column DNA digestion using RNase-free DNase kit (Qiagen, cat\# 79254). RNA was eluted in RNase free water and stored at $-80^{\circ} \mathrm{C}$.

Up to $1 \mu \mathrm{g}$ of the total RNA was reverse transcribed using the First Strand cDNA Synthesis Kit (Thermo Scientific, cat\# 10774691) with random primers according to the manufacturer's instructions. In 96-well format, real-time quantitative PCR (RT-qPCR) was carried out in $20-\mu \mathrm{L}$ reaction containing $10 \mathrm{ng}$ transcribed cDNA, SsoAdvanced ${ }^{\mathrm{TM}}$ Universal SYBR $^{\circledR}$ Green Supermix (Bio-Rad, cat\# 1725271) up to $0.5 \mathrm{nM}$ of each primer pair (Table 2.18) in technical duplicates on a CFX96 Touch Real-Time PCR Detection System (BioRad). In 384-well format, RT-qPCR was carried out in 10- $\mu \mathrm{L}$ reaction containing $10 \mathrm{ng}$ transcribed cDNA, SYBR ${ }^{\circledR}$ Select Master Mix (Thermo Fischer Scientific, cat\# 4472908) and up to $0.5 \mathrm{nM}$ of each primer pair (Table 2.18) in technical triplicates on a Quantstudio $12 \mathrm{~K}$ Flex system (Thermo Fischer Scientific). The used qPCR programs are described below.

\begin{tabular}{|c|c|c|c|c|c|c|}
\hline 96-well forma & & & 384-well for & & & \\
\hline $95^{\circ} \mathrm{C}$ & $1 \mathrm{~min}$ & & $95^{\circ} \mathrm{C}$ & $10 \mathrm{~min}$ & & activation \\
\hline $95^{\circ} \mathrm{C}$ & $15 \mathrm{sec}$ & & $95^{\circ} \mathrm{C}$ & $15 \mathrm{sec}$ & & denaturation \\
\hline $60^{\circ} \mathrm{C}$ & $15 \mathrm{sec}$ & 40 cycles & $60^{\circ} \mathrm{C}$ & $1 \mathrm{~min}$ & 40 cycles & $\begin{array}{l}\text { annealing/ex- } \\
\text { tension }\end{array}$ \\
\hline $60^{\circ} \mathrm{C}-95^{\circ} \mathrm{C}$ & $15 \mathrm{sec}$ & & $60^{\circ} \mathrm{C}-95^{\circ} \mathrm{C}$ & $15 \mathrm{sec}$ & & melting curve \\
\hline
\end{tabular}


Relative gene expression levels in wild type and reporter THP-1 М $\varphi$ were normalized to the MO (PMA-differentiated, non-polarized, non-treated) control group using the $2^{-\triangle \Delta C t}$ method. ${ }^{209}$ The ribosomal protein L37A (RPL37A) and ß-actin (ACTB) proteins served as a housekeeping genes ${ }^{210}$ for $4 \mathrm{~h}$ and $24 \mathrm{~h}$ time points, respectively.

Table 2.18: Primer pairs used in RT-qPCR.

*Primer pairs designed and validated by Dr. Hiromi Shiratori, Fraunhofer IME-TMP, Frankfurt am Main.

\begin{tabular}{|c|c|c|c|}
\hline gene & primer pairs ( $5^{\prime}$ to $3^{\prime}$ orientation) & primer conc. (nM) & primer efficiency (\%) \\
\hline \multirow{2}{*}{ CEBPD } & forward: CAG CAA CGA CCC ATA CCT CA & \multirow{2}{*}{350} & \multirow{2}{*}{102.3} \\
\hline & reverse: TCT TTG CGC TCC TAT GTC CC & & \\
\hline \multirow{2}{*}{ RPL37A } & forward: CTC GTC CGC CTA ATA CCG C & \multirow{2}{*}{425} & \multirow{2}{*}{100.6} \\
\hline & reverse: TAC CGA CGA TCC CGA CTT TC & & \\
\hline \multirow{2}{*}{ ACTB* } & forward: ATT GCC GAC AGG ATG CAG AA & \multirow{2}{*}{400} & \multirow{2}{*}{98.7} \\
\hline & reverse: GCT GAT CCA CAT CTG CTG GAA & & \\
\hline \multirow{2}{*}{ SEAP } & forward: GAG ATG AGT TTT TGT TCA CCC G & \multirow{2}{*}{500} & \multirow{2}{*}{110.0} \\
\hline & reverse: GAC CTT CAT AGC GCA CGT CA & & \\
\hline \multirow{2}{*}{ CCL2* } & forward: AAA CTG AAG CTC GCA CTC TCG C & \multirow{2}{*}{500} & \multirow{2}{*}{97.03} \\
\hline & reverse: AGG TGA CTG GGG CAT TGA TTG & & \\
\hline \multirow{3}{*}{$I L-6^{*}$} & forward: GTG TGA AAG CAG CAA AGA GGC & \multirow{3}{*}{400} & \multirow{3}{*}{90.6} \\
\hline & reverse: TCT GTT CTG GAG GTA CTC TAG & & \\
\hline & GTA T & & \\
\hline \multirow{3}{*}{$I L-1 \beta^{*}$} & forward: GTG GCA ATG AGG ATG ACT TGT & \multirow{3}{*}{400} & \multirow{3}{*}{93.47} \\
\hline & $T C T$ & & \\
\hline & reverse: TGT AGT GGT GGT CGG AGA TTC G & & \\
\hline
\end{tabular}

All primers were designed for human mRNA sequences covering exon-exon junctions, except for CEBPD (an intronless gene). RPL37A primer pair was exclusively designed to detect gDNA contamination (chapter 8.6.4, Fig.8-15). Primer efficiencies were verified in 96-well format, under mentioned conditions for RT-qPCR.

\subsection{ER-stress treatment}

Thapsigargin, a SERCA inhibitor, ${ }^{211}$ was used to induce endoplasmic reticulum stress (ERstress) in HEK293T reporter cells. In short, HEK293T wild type cells were seeded on glass cover slips in a 6-well format and transiently transfected (chapter 2.28.1) with SP-SEAP-myc-NLS-mCherry encoding constructs (for cloning strategy see chapter 8.8.2; Fig.8-32 to Fig.8-36). Next day after transfection, the overnight cellular supernatants 
were replaced by fresh cell culture medium containing $3 \mu \mathrm{M}$ thapsigargin and incubated for 6 hours. The thapsigargin treated cells were examined via Western Blotting (chapter 2.29) and immunofluorescence (chapter 2.30) analysis as well as via SEAP enzymatic assay (chapter 2.34.1.1). DMSO-treated cell were used as negative control.

\section{$1.5 \mathrm{mM}$ thapsigargin stock solution}

$1 \mathrm{mg}$ thapsigargin (Adipogen, cat\# AG-CN2-0003)

$1 \mathrm{~mL}$ DMSO

stored aliquoted at $-20^{\circ} \mathrm{C}$

\subsection{Enzymatic reporter assay}

Secreted enzymatic SEAP and GLuc gene reporters were detected in cellular supernatants of HEK293T and THP-1 reporter cells. After cell debris were removed by centrifugation for 2 minutes at $16,200 \times \mathrm{g}$, the cleared supernatants were stored at $-20^{\circ} \mathrm{C}$.

\subsubsection{SEAP assay}

SEAP utilizes three different substrates enabling its chemiluminescent, fluorescent, and colorimetric detection. To eliminate possible bias through the enzymatic signals produced by the endogenous SEAP-like proteins, the collected cellular supernatants were heat-inactivated for 30 minutes at $65^{\circ} \mathrm{C}$ prior measurement. ${ }^{16}$

\subsubsection{Chemiluminescent SEAP assay}

96-well format

Chemiluminescent SEAP assay protocol was adapted from Phospha-Light ${ }^{\text {TM }}$ SEAP Kit (Applied Biosystems ${ }^{\mathrm{TM}}$, Thermo Fischer Scientific, cat\# T1016) protocol. In short, heat-inactivated cellular supernatants were diluted 1:1 (v/v) in kit provided dilution buffer. On the plate (white, Greiner Bio-One, cat\# 655098, 655083), $50 \mu \mathrm{L}$ of assay buffer were added to $50 \mu \mathrm{L}$ of diluted sample and incubated for 5 minutes at room temperature. The enzymatic reaction was started by addition of $50 \mu \mathrm{L}$ of substrate buffer and immediately monitored using EnSpire multimode plate reader for 25 to 45 minutes ( $0.1 \mathrm{sec} /$ well). Cellular supernatants of wild type THP-1 cells or HEK293T cells, stably expressing backbone vector were used as a negative control. 
384-well format

During HTS for CEBPD-modulating compounds, chemiluminescent SEAP assay was performed in a 384-well format (white plate, Greiner Bio-One, cat\# 781904). Here, the routinely used chemiluminescent SEAP protocol in 96-well format was down-scaled according to the new developed protocol with $10 \mu \mathrm{L}$ of diluted sample (1:1 dilution with a supplied dilution buffer), $10 \mu \mathrm{L}$ of assay buffer, and $10 \mu \mathrm{L}$ of substrate buffer per well.

\subsubsection{Fluorescent SEAP assay}

Fluorescent SEAP assay was performed using Alkaline Phosphatase Detection Kit (Sigma Aldrich, cat\# APF-1KT), according to the manufacturer's instructions. Shortly, $20 \mu \mathrm{L}$ of heat-inactivated cellular supernatants were placed on the 96-well plate (black, Greiner Bio-One, cat\# 655090) and diluted with $20 \mu \mathrm{L}$ of supplied dilution buffer, followed by the addition of $160 \mu \mathrm{L}$ fluorescent assay buffer. The enzymatic reaction was started by addition of $1 \mu \mathrm{L}$ of $10 \mathrm{mM}$ 4-MPP substrate buffer and immediately monitored using EnSpire multimode plate reader at $360 \mathrm{~nm}$ excitation and $440 \mathrm{~nm}$ emission wavelengths for 25 to 45 minutes.

$10 \mathrm{mM}$ 4-methylumbelliferyl phosphate disodium salt (4-MPP)

$1 \mathrm{mg} \quad 4-\mathrm{MPP}$ (supplied in Kit)
$330 \mathrm{\mu L} \quad \mathrm{dd}_{2} \mathrm{O}$
stored aliquoted at $-20^{\circ} \mathrm{C}$

\subsubsection{Colorimetric SEAP assay}

Colorimetric SEAP assay protocol was adopted from Berger et al. ${ }^{16}$ In short, $10 \mu \mathrm{L}$ of the heat-inactivated cellular supernatants were mixed with $90 \mu \mathrm{L} \mathrm{ddH}_{2} \mathrm{O}$ and $100 \mu \mathrm{L}$ SEAP buffer on a 96-well plate (transparent, Greiner Bio-One, cat\# 655101) and incubated for $10 \mathrm{~min}$ at $37^{\circ} \mathrm{C}$. Immediately after substrate buffer addition, the absorption of the reaction was measured at $405 \mathrm{~nm}$ for 25 to 45 minutes using EnSpire multimode plate reader. 
SEAP buffer

$\begin{array}{ll}1 \mathrm{M} & \text { diethanolamine } \\ 10 \mathrm{mM} & \text { L-homoarginine-hydrochloride } \\ 0.5 \mathrm{mM} & \mathrm{MgCl}_{2}\end{array}$

SEAP substrate solution

$120 \mathrm{mM}$ p-nitrophenyl phosphate,

dissolved in SEAP buffer

\subsubsection{GLuc assay}

Chemiluminescent GLuc assay was performed as described previously. ${ }^{212}$ In short, $15 \mu \mathrm{L}$ of cellular supernatants were placed on plate (white, Greiner Bio-One, cat\# 655083 and incubated for $5 \mathrm{~min}$ at $37^{\circ} \mathrm{C}$. The $20 \mu \mathrm{M}$ coelenteracine substrate buffer was prepared in assay buffer and incubated for $30 \mathrm{~min}$ at room temperature protected from light. The enzymatic reaction was started by injection of $50 \mu \mathrm{L}$ substrate buffer and immediately monitored using EnSpire multimode plate reader for 2 minutes (10 seconds/well).

$\underline{\text { GLuc assay buffer }}$

$5 \mathrm{mM} \mathrm{NaCl}$ in PBS

$\mathrm{pH} 7.2$

stored at $4^{\circ} \mathrm{C}$

coelenteracine substrate buffer

$20 \mu \mathrm{M}$ coelenteracine in assay buffer

prepared freshly

\section{$5.9 \mathrm{mM}$ coelenteracine stock solution}

2.5 mg coelenteracine (Carl Roth, cat\# 4094)

$1 \mathrm{~mL}$ ethanol

stored aliquoted at $-80^{\circ} \mathrm{C}$ protected from light

\subsection{SEAP and GLuc assay validation parameters}

For chemiluminescent SEAP and GLuc gene reporter assays following validation parameters were determined: assay sensitivity, linearity, and variability. Chemiluminescent SEAP assay was validated using CMV::SEAP HEK293T reporter cells for assay performance in 96-well format, and THP-1 reporter cells - for 384-well assay format. Chemiluminescent GLuc assay was validated using CMV::Gaussia HEK293T reporter cells for assay performance in 96-well format. Intra- and inter-assay coefficient of variations as well as Z'-factor value were calculated using HEK293T cells, stably expressing backbone vector, or wild type THP-1 cells as negative control. The Z'-factor, a statistical factor for assay quality in the setting of high throughput screening, ${ }^{213}$ was calculated as follows: 
$\mathrm{Z}^{\prime}=1-\left(\frac{3 *(\mathrm{SD} \text { (sample })+\mathrm{SD}(\text { neg. control }))}{\mid \text { mean (sample) }- \text { mean (neg. control) } \mid}\right)$

SD (sample) : standard deviation of the sample;

SD (neg. control): standard deviation of negative control.

\author{
$1 \geq Z^{\prime}$-factor $\geq 0.5$ : excellent assay; \\ $0>Z^{\prime}$-factor $>0.5$ : double assay; \\ $Z^{\prime}$-factor = 0: “yes/no" type assay; \\ $Z^{\prime}$-factor < 0: screening is impossible.
}

\subsection{Compound preparation}

Screening compounds of LOPAC $^{\circledR 1280}$ and $\mathrm{ENZO}^{\circledR 774}$ libraries were maintained in DMSO at $10 \mathrm{mM}$ and stored on 384-well plates (Echo Qualified 384-well plates, Labcyte) at $80^{\circ} \mathrm{C}$ (Fraunhofer IME ScreeningPort, Hamburg). Before screening, compounds were thawed at room temperature and applied at final concentration of $10 \mu \mathrm{M}$. Stock solutions of recombinant human IFN-g $(1 \mathrm{mg} / \mathrm{mL})$ and LPS $(1 \mathrm{mg} / \mathrm{mL})$ were prepared in cell culture-grade DPBS. Stock solutions of compounds GSK 1210151A (10 mM), Ro 11-1464 $(10 \mathrm{mM})$, Trichostatin A $(6.6 \mathrm{mM})$, and Vorinostat $(10 \mathrm{mM})$ were prepared in DMSO. IFN$\mathrm{g}$ stock solution and all of the remaining stock solutions were stored at $-80^{\circ} \mathrm{C}$ and $-20^{\circ} \mathrm{C}$, respectively. The desired compound concentrations were freshly prepared in the cell culture medium for each treatment.

\subsection{In vitro differentiation of THP-1 cells}

In order to obtain THP-1-derived $\mathrm{M} \varphi$, wild type and reporter THP-1 cells were seeded in desired plate format and stimulated for 48 hours with $50 \mathrm{ng} / \mathrm{mL}$ PMA, followed by further incubation in fresh cell culture medium for 72 hours, as described previously. ${ }^{214}$ During screening, THP-1 target reporter cells were differentiated in a bulk format (chapter 2.39.1).

\subsection{M1 treatment: in vitro polarization of THP-1 cells}

For M1 polarization, differentiated THP-1 wild type and reporter cells were co-stimulated with LPS $(0.1$ or $1 \mu \mathrm{g} / \mathrm{mL})$ and IFN-g $(20 \mathrm{ng} / \mathrm{mL})$, as described previously. ${ }^{214}$ The gene expression was investigated in cells incubated with stimuli for 1, 3, and 6 hours. For SEAP enzymatic assay, the cells were M1 treated for 24 hours. 


\subsection{Screening for CEBPD-modulating compounds}

\subsubsection{High-throughput screening}

HTS for CEBPD-modulating compounds was performed in a 384-well format semi-automatically at Fraunhofer IME ScreeningPort in Hamburg. LOPAC ${ }^{\circledR 1280}$ and ENZO ${ }^{\circledR 74}$ compound libraries, containing 1280 and 774 compounds respectively, were screened twice. THP-1 reporter cells were differentiated in a bulk format: cells were seeded in T175 flasks with 12 million cells and stimulated with $50 \mathrm{ng} / \mathrm{mL}$ PMA for 48 hours, followed by cell recovery in fresh cell culture medium without PMA for 72 hours. PMA-differentiated THP-1 reporter $M \varphi$ were detached using Accutase ${ }^{\circledR}$ solution (Sigma-Aldrich, cat\# A6964) for 1 hour at $37^{\circ} \mathrm{C}$, centrifuged for $5 \mathrm{~min}$ at $300 \times \mathrm{g}$ and seeded in a 384-well format with 10,000 cells per well in $50 \mu \mathrm{L}$ of RPMI 1640 cell culture medium without phenol red. Next day, overnight cell culture medium was automatically removed and $20 \mu \mathrm{L}$ of fresh medium were manually added to the re-attached cells. Next, cells were pre-treated with screening and TSA control compounds, added robotically $(0.2 \mu \mathrm{L}$ per well, Echo 550 Liquid Handler) for 1 hour at $37^{\circ} \mathrm{C}$. Pre-treated cells were stimulated by manually addition of $20 \mu \mathrm{L}$ cell culture medium containing $0.1 \mu \mathrm{g} / \mathrm{mL}$ LPS and $20 \mathrm{ng} / \mathrm{mL} \mathrm{IFN-g} \mathrm{(M1} \mathrm{treat-}$ ment, chapter 2.37). The final screening compound $(10 \mu \mathrm{M})$ and TSA $(0.5 \mu \mathrm{M})$ concentrations were restored by the second robotic compound addition. Finally, cell culture medium of treated cells was robotically collected using JANUS Mini Platform (Perkin Elmer), 24 hours after M1 treatment and analysed via SEAP readout assay. Chemiluminescent SEAP assay was performed in 384-well format (chapter 2.34.1.1) on Envision plate reader (Perkin Elmer). Cells were analysed via CellTiter-Glo ${ }^{\circledR}$ cell viability assay (chapter 2.39.3).

\subsubsection{Hit compound confirmation}

Hit compound confirmation was performed manually according to the screening protocol described previously (chapter 2.39.1). THP-1 reporter cells were PMA-differentiated in a bulk format and cultured in a 96-well format with 37,000 cells per well in $100 \mu \mathrm{L}$ of RPMI 1640 cell culture medium without phenol red. Chemiluminescent SEAP assay was performed in a 384-well format (chapter 2.34.1.1). Obtained SEAP signals were normalized to the corresponding cell numbers, determined via CellTiter-Glo ${ }^{\circledR}$ assay (chapter 2.39.3). 


\subsubsection{Cell viability assay}

Cell viability assay was performed using CellTiter-Glo ${ }^{\circledR}$ Kit (Promega, cat\# G7570) in 384and 96-well format according to the manufacturer's instructions. Shortly, after cellular supernatants were collected for SEAP assay, freshly prepared CellTiter-Glo ${ }^{\circledR}$ reagent was added to the cells with $100 \mu \mathrm{L}$ per well and mixed for 2 minutes on an orbital shaker to induce cell lysis. Upon incubation for 10 minutes at room temperature, luminescence was recorded on EnSpire plate reader with $0.25 \mathrm{sec} /$ well integration time.

\subsubsection{Hit compound characterization}

Characterization of GSK 1210151A, Ro 11-1464, vorinostat, and trichostatin A hit compounds was performed manually according to the screening protocol described previously (chapter 2.39.1). THP-1 reporter cells were PMA-differentiated in a bulk format and cultured in a 24-well format with 120,000 cells per well in $0.5 \mathrm{~mL}$ of RPMI 1640 cell culture medium without phenol red. Hit compound-mediated effects on SEAP secretion and expression of selected genes (Table 2.18) was determined via chemiluminescent SEAP assay (384-well format, chapter 2.34.1.1), and real-time quantitative PCR (chapter 2.32), respectively. The compound-mediated effect on SEAP secretion was determined for all 18 selected compounds, to analyse signal correlation (chapter 8.6.3 Fig.8-14).

\subsection{Statistical analysis}

All independent experiments were repeated at least three times. Significant differences were evaluated using GraphPad Prism 8.0.1 software. The criteria for significance were set with the following $p$-value: ${ }^{*} p<0.05 ;{ }^{* *} p<0.005{ }^{* * *} p<0.001 ; * * p<0.0001$. 


\section{Results}

\subsection{Screening assay design and experimental workflow}

Development of phenotypic screening assay starts with definition of suitable screening setting that includes three criteria - stimulus, cellular system, and readout. As the suitability of these criteria depends on their relevance in the chosen disease context (cf. chapter 1.1), I set stimulus, cellular system, and readout regarding to the inflammatory aspects of RA (cf. chapter 1.3.3). In this study, LPS and IFN-g serve as the screening stimuli, M1-polarized THP-1 reporter $\mathrm{M} \varphi$ - as the cellular system, and CEBPD as the target gene for the screening readout.

Bacterial artificial chromosome (BAC) that encodes a piece $(201,427 \mathrm{bp})$ of the human chromosome 8 including CEBPD gene was a genetic source of CEBPD promoter sequence, which I confirmed by sequencing (chapter 8.1.1, Fig.8-1). To monitor changes in CEBPD target promoter activity I used SEAP as a gene reporter, which I combined with further reporter genes such as GLuc and eGFP to a multi-gene-reporter cassette 1.0 (Fig.3-1A). I stably integrated this cassette, which was expressed on a viral vector, into the genome of THP-1 cells. Generated THP-1 reporter $\mathrm{M} \varphi$ that undergo polarization toward pro-inflammatory M1 state in response to LPS and IFN-g treatment I used for the HTS for CEBPD-modulating compounds.

The THP-1-derived $\mathrm{M} \varphi$ display multiple advantages of their use during HTS: i) their easy handling and acquisition, ii) no limitation in cell number, iii) their homogeneous genetic background as well as purity of the obtained $M \varphi$ population. ${ }^{197}$ M1-polarized THP-1 M $\varphi$ display certain gene and surface protein expression markers that are comparable to that of M1-polarized peripheral blood mononuclear cells (PBMCs). ${ }^{214}$

During screening, monitoring of compound-mediated changes in CEBPD promoter activity relies on usage of a robust, stable, sensitive, and non-invasive gene reporter like SEAP. As introduced previously (cf. chapter 1.2), SEAP is an enzymatic and secreted reporter protein, whose transcription is initiated upon activation of a target promoter (Fig.3-1B). Thus, SEAP indicates activation of CEBPD promoter by a strong enzymatic signal detected in the cellular supernatant (Fig.3-1B), which otherwise happens unperceived in the nucleus. In THP-1 reporter $M \varphi$, the obtained SEAP signals can be norma- 
A

start (0)

locus human CEBPD: BAC clone CH17-293N3

stop $(201,427)$

location: 94,973..96,223 complement

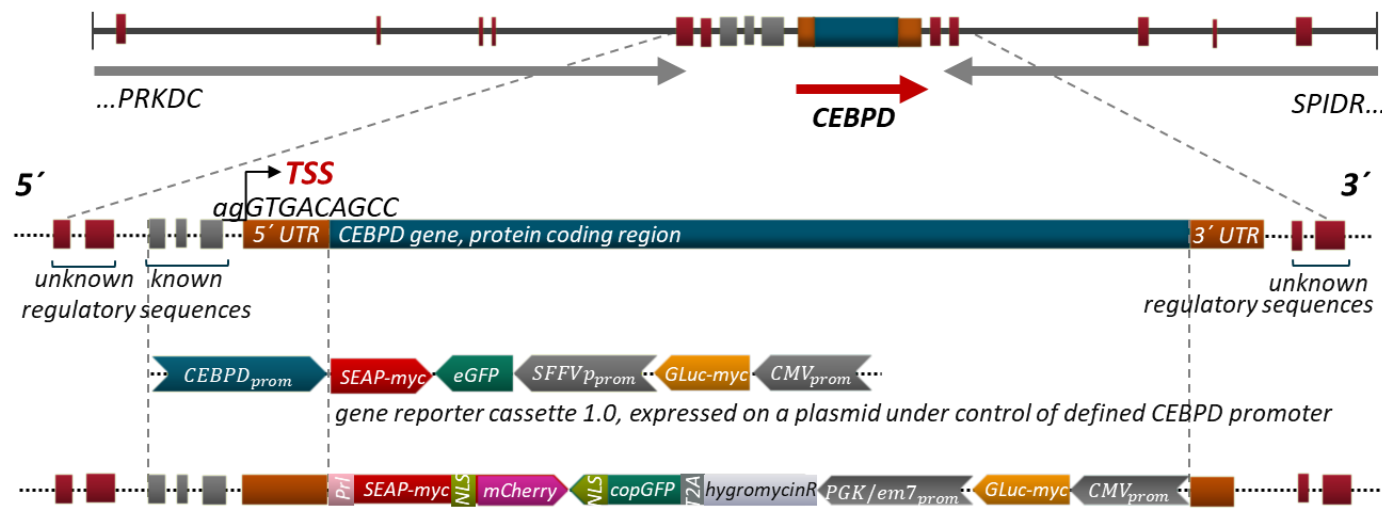

gene reporter cassette 2.0, supposed to be expressed on BAC under control of endogenous CEBPD promoter

promoter protein
on forward DNA strand
on complementary DNA strand

B

C

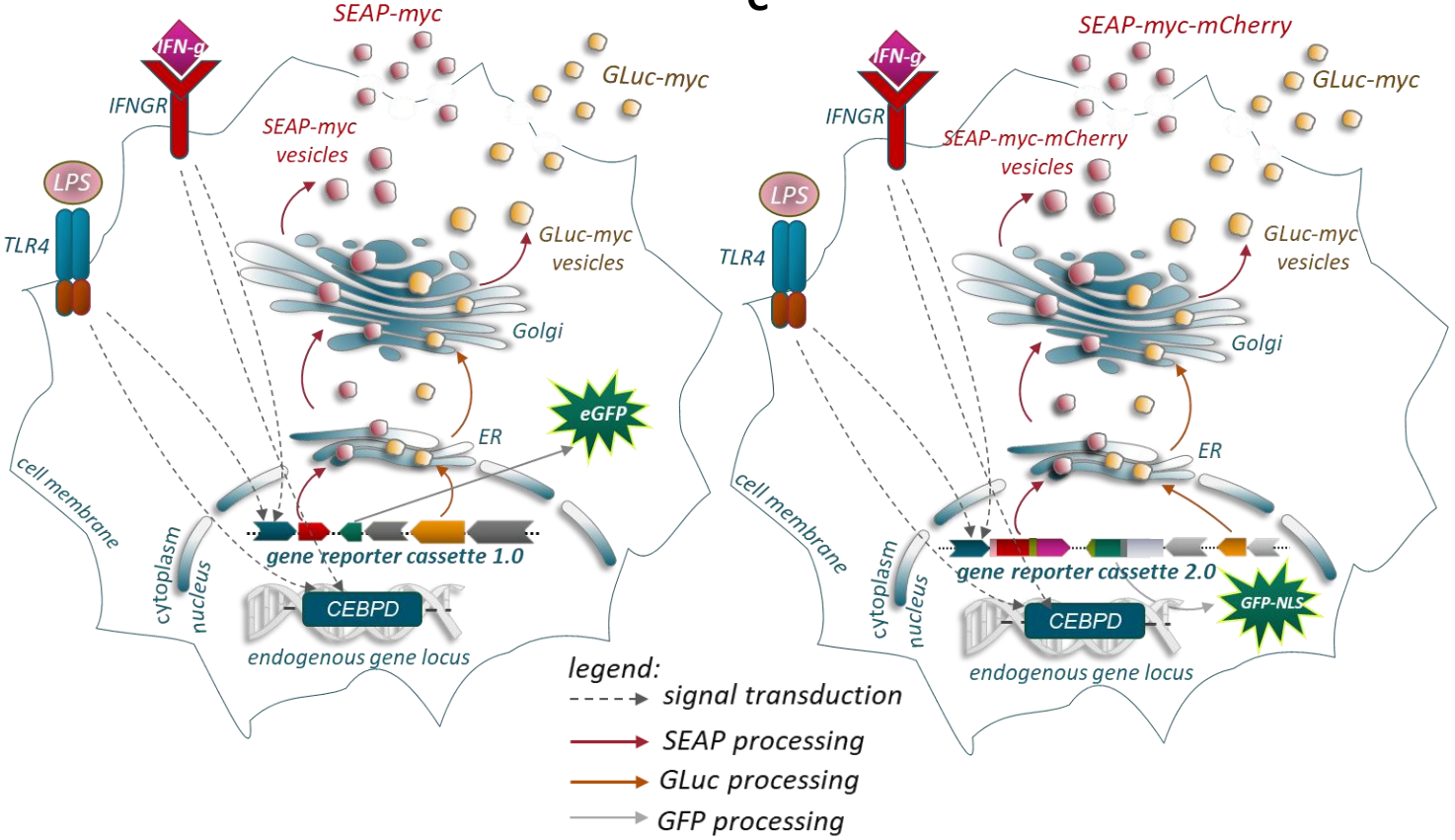

Figure 3-1: Structure of generated gene reporter cassettes and principles of their functioning in the cell. A: BAC clone CH17-293N3 that encodes a fragment (201,427 bp) of human chromosome 8 , including CEBPD (red arrow), SPIDR, and PRKDC (grey arrows) genes, served as a genetic source for CEBPD target promoter. Expressed on a plasmid (viral vector), multi-gene-reporter cassette 1.0 encodes SEAP-myc under control of defined CEBPD promoter, restricted to contain only the most proximal known regulatory sequences, TATA box, and 5 ' UTR. Supposed to be expressed directly on BAC, multi-gene-reporter cassette 2.0 encodes modified Prl-SEAP-myc-NLS-mCherry fusion protein, which is thought to be set under control of endogenous CEBPD promoter, containing known and unknown regulatory sequences. Both cassettes further encode GLuc-myc enzymatic and GFP fluorescent gene reporters under control of constitutive promoters. B: In M1-polarized THP-1 reporter M $\varphi$ stably expressing multi-gene-reporter cassette 1.0, LPSand IFN-g treatment activates CEBPD promoter resulting in secretion of SEAP-myc, whose enzymatic activity is detected in cellular supernatant. Constantly secreted GLuc-myc reporter protein may serve as an internal control. Cytoplasmic eGFP was used to select positive clones. C: Modified PrI-SEAP-myc-NLSmCherry (SEAP-myc-mCherry) fusion protein, stably expressed in M1-polarized THP-1 reporter M $\varphi$, could be used for both monitoring of CEBPD promoter activity and visual detection of ER-stress. NLS-tagged copGFP exclusively locates in the nucleus supporting visual ER-stress detection. GLuc-myc may serve as an internal control. 
lized to the corresponding signals of GLuc - a naturally secreted luciferase. As GLuc is expressed under control of a strong constitutive promoter CMV, it is constantly secreted into the cellular supernatant and can therefore serve as an internal control (Fig.3-1B). The multi-gene-reporter cassette 1.0 encodes CEBPD target promoter that was restricted to the most proximal, known regulatory sequences, TATA box, and $5^{\prime}$ UTR (Fig.1-3A,B). Such simplification relied on a limited cloning capacity of the viral vector, on which the cassette 1.0 was expressed, and on lack of knowledge about location and functionality of further CEBPD regulatory sequences in the proximal promoter region (cf. chapter 1.3.1.3). To enhance screening reliability, I proposed to express gene reporter cassette under control of the endogenous CEBPD promoter directly on BAC that

1. Selection of a gene reporter for screening readout

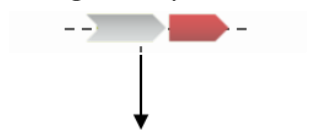

2. Selection of a gene reporter for normalization

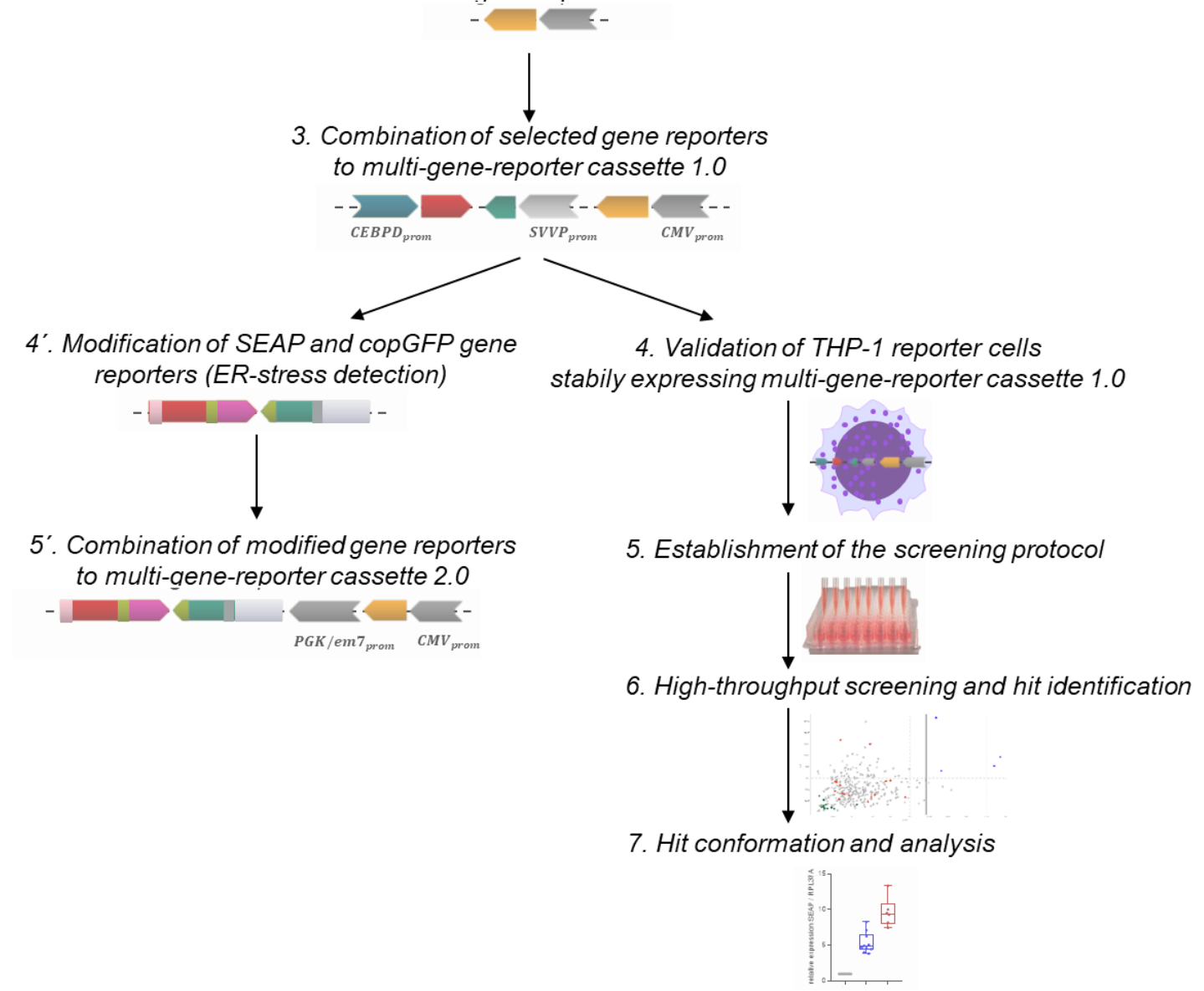

Figure 3-2: Experimental workflow - from screening assay development to the hit compound characterization. This 7-step experimental workflow gives an overview of the whole practical part. In this study, the main focus was on screening assay development and execution of HTS for CEBPD-modulating compounds (steps 1-7). A side-project (steps 4' and 5') focused on generation of a multi-gene-reporter cassette 2.0, to be expressed on BAC (development of cloning strategy). 
covers CEBPD's genomic area of about 200,000 base pairs. Here, I planned to use an optimized multi-gene-reporter cassette 2.0 encoding modified SEAP and GFP reporter proteins (Fig.3-1A). The use of this cassette should enable both monitoring of CEBPD promoter activity and visual detection of a cellular stress (Fig.3-1C), which can impair SEAP secretion and therefore cause data misinterpretation. Although I could show that the multi-gene-reporter cassette 2.0 was functional in HEK293T cells, its expression on BAC in THP-1 target cells remained a future task.

In summary, the priority of this study was to develop and conduct of a HTS for CEBPDmodulating compounds in THP-1 reporter $\mathrm{M} \varphi$ followed by characterization of identified hit compounds (Fig.3-2). Generation of the multi-gene-reporter cassette 2.0 and testing of its functionality belonged to a side-project, further development of which exceeded the time limitation of this study (Fig.3-2).

\subsection{Development of the screening assay}

\subsubsection{Generation and characterization of the multi-gene-reporter cassette $\mathbf{1 . 0}$}

3.2.1.1 Selection of gene reporters suitable for monitoring of CEBPD promoter activity or for normalization

Initially, it was important to determine which reporter protein, SEAP or GLuc, suits at its best to monitor CEBPD gene expression and which can serve as an internal control. Selection of the best suitable reporter protein relied on characterization of the corresponding enzymatic assay. Therefore, I generated three HEK293T reporter cell lines that stably expressed each of the reporter-encoding constructs or an empty vector (Fig.3-3A). Generation of stably transfected HEK293T reporter cells displayed certain advantages over transient transfection: i) no transient transfection variation; ii) no acute cellular stress via transfection reagent addition; iii) easy handling of HEK293T cells; iv) no limitation in cell number and cellular supernatant volume. SEAP and GLuc gene reporters displayed their expected enzymatic activities in cellular supernatants of corresponding HEK293T reporter cells (Fig.3-3B, D). SEAP and GLuc proteins carried a C-terminal myc-tag that enabled their immunological detection via Western Blotting (Fig.3-3C) and immunohistochemistry (Fig.3-3E). SEAP and GLuc showed predominantly 
A
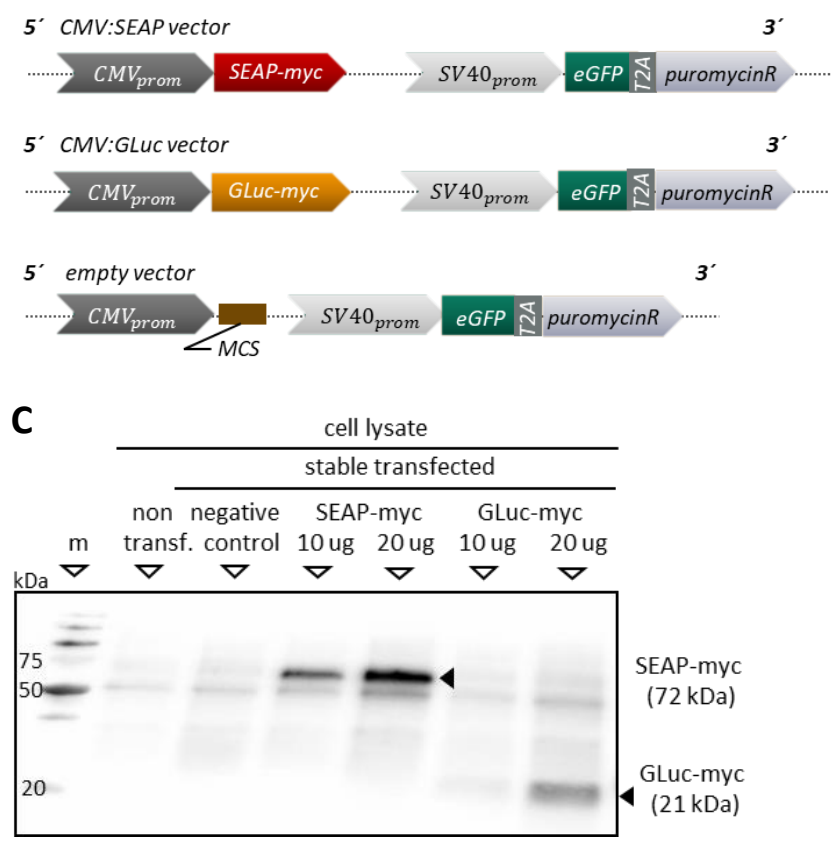

E
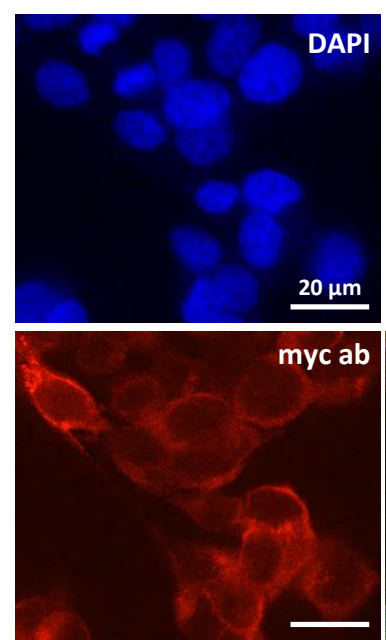
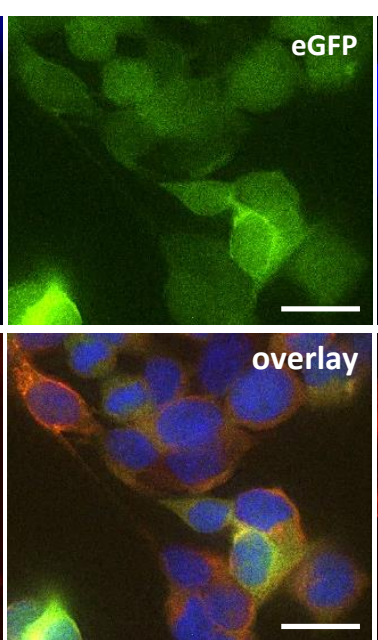

B

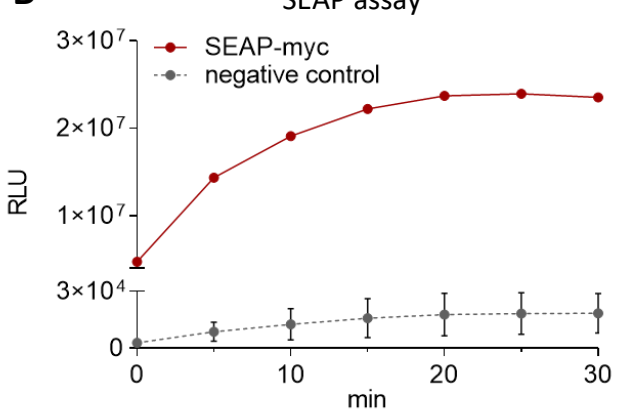

D

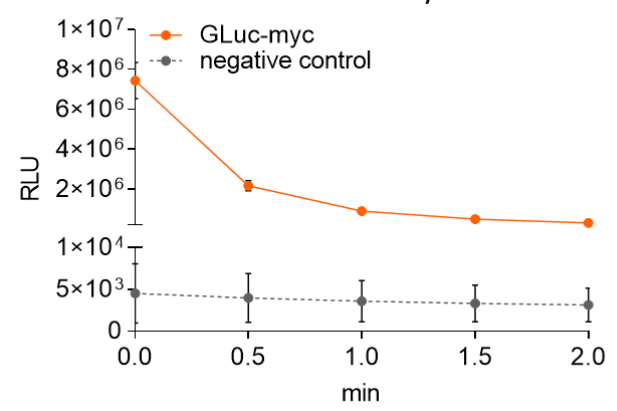

GLuc-myc
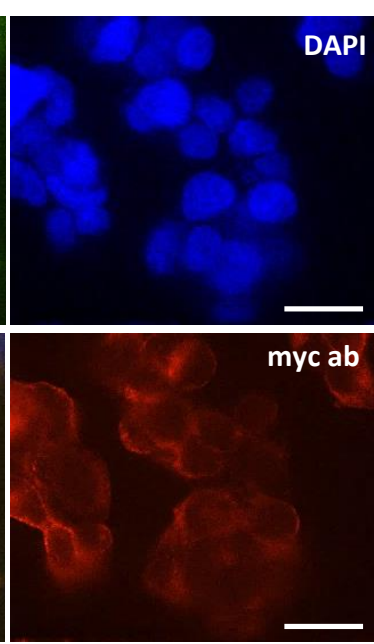
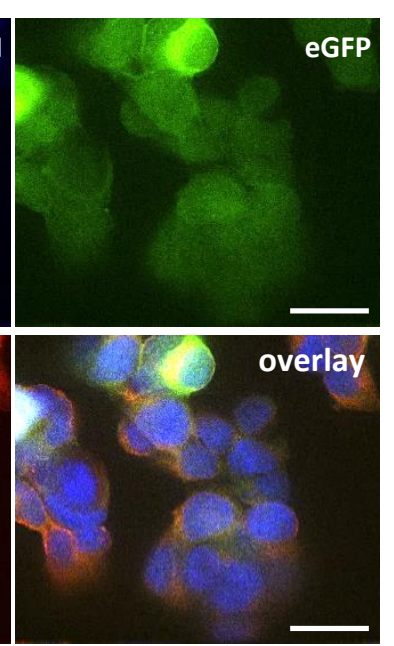

Figure 3-3: Characterization of HEK293T reporter cells stably expressing SEAP or GLuc. Following stable transfection with CMV::SEAP, CMV::GLuc or empty vectors (A), HEK293T cells were cultured on object slides in a 6-well format for 24 hours. Enzymatic activity of each of the reporters was measured in cellular supernatants of corresponding cells $(B, D)$. Cells attached to object slides were used for immunofluorescence analysis (E) with the remaining cells being lysed for Western Blotting (C). Cellular supernatants (B, D) or protein extracts (C) from empty vector-expressing HEK293T cells were utilized as negative control. A: Schematic drawing of generated constructs. Myc-tagged SEAP or GLuc reporters were cloned into empty vector encoding G2P fusion protein. G2P contains eGFP, which is linked via T2A self-cleavage peptide to a puromycin resistance gene (encodes puromycin $\mathrm{N}$-acetyl-transferase; puromycinR). MCS: multiple cloning site B, D: Enzymatic activity of secreted SEAP-myc and GLuc-myc gene reporters. Each point represents average signal \pm SD of three independent experiments. RLU: relative luminescence units C: Western Blotting analysis of protein extracts from non-transfected (non transf.) and stable transfected HEK293T cells. Detected with an anti-c-myc antibody (1:500, Sigma Aldrich), SEAP-myc and GLuc-myc displayed expected protein sizes at 72 and $21 \mathrm{kDa}$, respectively. m: protein ladder. E: Immunofluorescence analysis of HEK293T reporter cells. SEAP-myc and GLuc-myc proteins were detected intracellularly using rabbit anti-c-myc (myc ab; 1:100, Sigma Aldrich) primary and anti-rabbit Alexa 555-conjugated secondary (1:1000, Thermo Fischer Scientific) antibodies. DAPI: nuclear staining. Scale bar: $20 \mu \mathrm{m}$. 
ex-nuclear localization in HEK293T reporter cells, typically for secreted proteins (Fig.3-3E).

The suitability of reporter proteins relied on the fulfilment of purpose-dependent criteria. During screening, monitoring of CEBPD gene expression required a very sensitive and temporarily flexible detection, as CEBPD displayed a relatively weak promoter strength (chapter 8.3, Fig.8-6) and timing of compound-mediated changes in CEBPD promoter activity was unknown. SEAP and GLuc reporters both displayed a high protein stability in cellular supernatants (cf. chapter 1.2) that led to their accumulation upon promoter activation and hence enabled temporally flexible detection. Here, it was more appropriate to compare the sensitivity of SEAP and GLuc enzymatic assays. Selection of suitable gene reporter for normalization relied on a high reporter protein stability as well as on a high assay repeat- and reproducibility.

Assay sensitivity, linearity, and variability ${ }^{215}$ I estimated in serially-diluted cellular supernatants of SEAP- (Fig.3-4) and GLuc-expressing HEK293T reporter cells (Fig.3-5). Assay sensitivity was determined by an end-point dilution, at which the assay could no longer distinguish between a positive (SEAP or GLuc) and a negative signal (background). The clear difference between a positive and a negative signal was shown by $Z^{\prime}$-factor, a statistical criterion that implicates means and signal deviations. ${ }^{213}$ An optimal Z'-factor for the SEAP and GLuc assay was set at $\geq 0.5$. Thus, chemiluminescent SEAP assay displayed high assay sensitivity indicated by the $Z^{\prime}$-factor with 0.7 for the 1,000-fold diluted sample (Fig.3-4B). In comparison, GLuc assay displayed only a moderate assay sensitivity as the highest dilution, at which $Z^{\prime}$-factor is $\geq 0.5$, was 100 (Fig.3-5B). Chemiluminescent SEAP assay was also the most sensitive in comparison to the colorimetric (chapter 8.2.1, Fig.8-3) and the fluorescent (chapter 8.2.2, Fig.8-4) SEAP assays.

Assay linearity is characterized by enzymatic signals that are directly proportional to the concentration of the enzyme, which decreases with raising dilution. Whereas SEAP assay was linear $\left(R^{2}>0.9900\right)$ over four 10-fold dilutions (Fig.3-4C), GLuc assay - over three 10-fold dilutions (Fig.3-5C).

Assay repeatability is expressed by a coefficient of variability (CV): the intra-assay CV and read-to-read CV. The intra-assay CV indicates the variability of enzymatic signals between the multiple technical replicates of the same sample. The read-to-read CV 

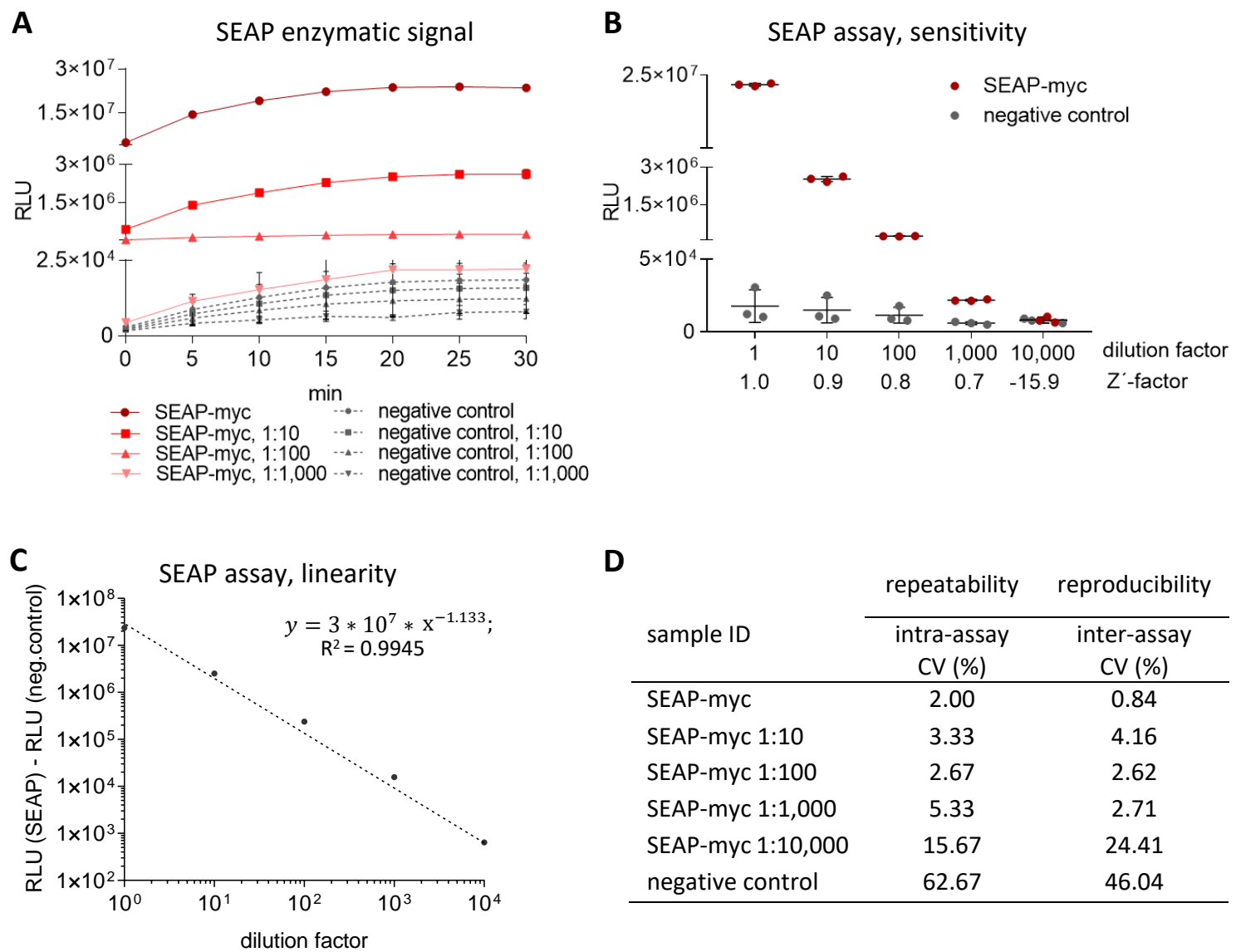

\begin{tabular}{lcc} 
D & repeatability & reproducibility \\
\cline { 2 - 3 } sample ID & $\begin{array}{c}\text { intra-assay } \\
\text { CV (\%) }\end{array}$ & $\begin{array}{c}\text { inter-assay } \\
\text { CV (\%) }\end{array}$ \\
\hline SEAP-myc & 2.00 & 0.84 \\
SEAP-myc 1:10 & 3.33 & 4.16 \\
SEAP-myc 1:100 & 2.67 & 2.62 \\
SEAP-myc 1:1,000 & 5.33 & 2.71 \\
SEAP-myc 1:10,000 & 15.67 & 24.41 \\
negative control & 62.67 & 46.04 \\
\hline
\end{tabular}

Figure 3-4: Characterization of the chemiluminescent SEAP assay. SEAP enzymatic activity was measured in undiluted and up to 10,000-fold diluted cellular supernatants of SEAP-expressing HEK293T reporter cells $24 \mathrm{~h}$ after cell seeding. Cellular supernatants of HEK293T cells stably transfected with empty vector were utilized as negative control. A: Kinetics of enzymatic reaction. SEAP readout was measured for each of the dilutions for 30 minutes after substrate addition. Each point represents the average signal \pm SD of three independent experiments. B: Determination of assay sensitivity. The highest dilution factor at which $Z^{\prime}$-factor is $\geq 0.5$ is 1,000 indicating high assay sensitivity. Data represent mean \pm SD of three independent experiments. C: Determination of assay linearity. Chemiluminescent SEAP assay is linear $\left(R^{2}=0.9945\right)$ over four 10-fold dilutions. Each point represents the average signal of three independent experiments, detected 20 minutes after substrate addition. D: Determination of assay repeatability and reproducibility. The intra- and inter-assay CVs were determined between the three technical replicates or the three independent experiments, respectively. RLU: relative luminescence units.

expresses the variability of enzymatic signals from the same sample detected on different assay plates. SEAP assay displayed low intra-assay CV (<10\%) for up to 1,000-fold diluted samples (Fig.3-4D), while GLuc assay showed moderate intra- and read-to-read CVs (<15\%) for up to 100 -fold diluted samples (Fig.3-5D). Assay reproducibility is expressed by the inter-assay CV that indicates the variability of enzymatic signals of samples that belong to different individual experiments. Chemiluminescent SEAP assay displayed low inter-assay CV (<5\%) for up to 1,000-fold diluted samples (Fig.3-4D) and thus a high assay reproducibility. GLuc assay showed moderate inter-assay CV (<15\%) for up to 100-fold diluted samples (Fig.3-5D). I also tested the suitability of the fluores- 
A

GLuc enzymatic signal

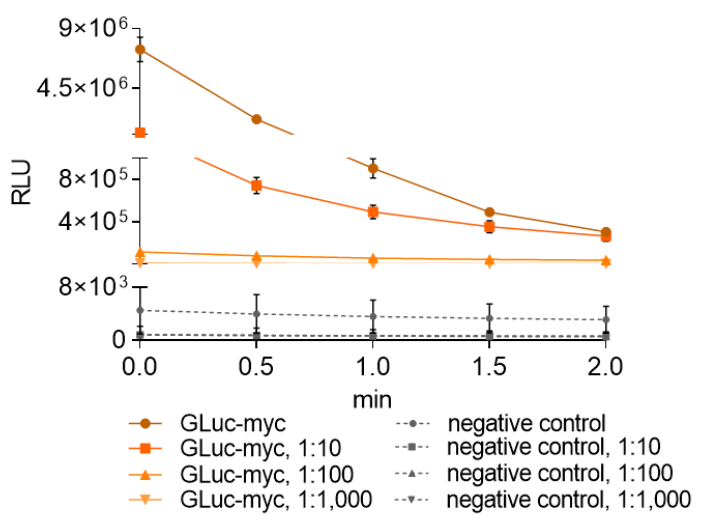

B
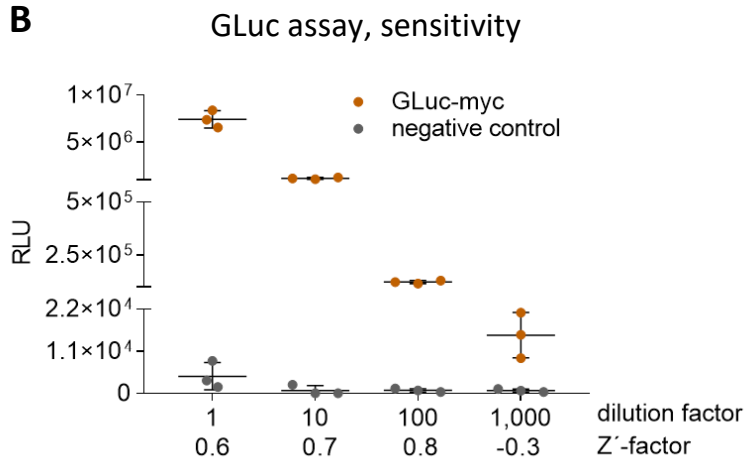

C

GLuc assay, linearity

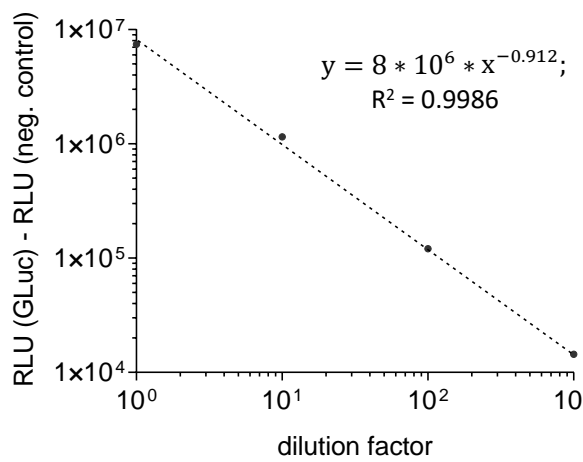

D

\begin{tabular}{lccc} 
& \multicolumn{2}{c}{ repeatability } & $\begin{array}{c}\text { reproduci- } \\
\text { bility }\end{array}$ \\
\cline { 2 - 4 } sample ID & $\begin{array}{c}\text { intra-assay } \\
\text { CV (\%) }\end{array}$ & $\begin{array}{c}\text { read-to-read } \\
\text { CV (\%) }\end{array}$ & $\begin{array}{c}\text { inter-assay } \\
\text { CV (\%) }\end{array}$ \\
\hline GLuc-myc & 13.0 & 13.77 & 12.29 \\
GLuc-myc 1:10 & 13.7 & 12.91 & 8.93 \\
GLuc-myc 1:100 & 7.9 & 12.14 & 5.97 \\
GLuc-myc 1:1,000 & 11.5 & 15.31 & 39.07 \\
negative control & 22.7 & 22.80 & 78.09 \\
\hline
\end{tabular}

Figure 3-5: Characterization of the chemiluminescent GLuc assay. GLuc enzymatic activity was measured in undiluted and up to 1,000-fold diluted cellular supernatants of GLuc-expressing HEK293T reporter cells $24 \mathrm{~h}$ after cell seeding. Cellular supernatants of HEK293T cells stably transfected with empty vector were utilized as negative control. A: Kinetics of enzymatic reaction. GLuc readout was measured for each of the dilutions for 2 minutes after substrate addition. Each point represents the average signal \pm SD of three independent experiments. B: Determination of assay sensitivity. The highest dilution factor at which $Z^{\prime}-$ factor is $\geq 0.5$ is 100 indicating moderate assay sensitivity. Data represent mean $\pm S D$ of three independent experiments. C: Determination of assay linearity. Chemiluminescent GLuc assay is linear $\left(R^{2}=0.9986\right)$ over three 10 -fold dilutions. Each point represents the average signal of three independent experiments, detected right after substrate addition. D: Determination of assay repeatability and reproducibility. The intra-assay, read-to-read, and inter-assay CVs were determined between the three technical replicates, between single measurements of same samples on three assay plates, or between the three independent experiments, respectively. RLU: relative luminescence units.

sent eGFP gene reporter to serve as internal control in THP-1 target cells. eGFP assay displayed low assay sensitivity, linearity, repeatability and reproducibility (chapter 8.2.3, Fig.8-5), eGFP was, therefore, an inappropriate gene reporter for normalization but could serve as a fluorescent cell marker.

In summary, SEAP was the more suitable reporter protein for monitoring of CEBPD promoter activity due to the higher assay sensitivity and broader linear range in comparison to these of the GLuc assay. GLuc gene reporter could function as an internal control due to its easy and temporary flexible detection and the acceptable inter-, read-to-read, and intra-assay CVs for up to 100 -fold diluted samples. 
3.2.1.2 Multi-gene-reporter cassette 1.0 is functional in target THP-1 cells

After assay characterization, I combined enzymatic SEAP and GLuc as well as fluorescent eGFP gene reporters to a multi-gene-reporter cassette 1.0 (Fig.3-6A). The inducible part of the cassette expressed SEAP under control of the defined CEBPD promoter (Fig.3-6B), while the constitutive part expressed GLuc and eGFP reporters under control of strong constitutive promoters CMV and SFFVp, respectively (Fig.3-6A).

To evaluate the use of SEAP and GLuc in target cells, I stably integrated the multi-genereporter cassette 1.0 into the genome of wild type THP-1 cells using a lentiviral vector. Following transduction and positive cell clone selection, I tested the differential plasticity of generated THP-1 reporter cells. Like wild type THP-1 cells, THP-1 reporter monocytes are suspension cells that differentiate to $M \varphi$ in response to the treatment with phorbol myristate acetate (PMA) changing their morphology and becoming adherent (Fig.3-6C). During their recovery in a fresh PMA-free medium, PMA-differentiated THP-1 M $\varphi$ were observed to express gene and cell surface protein markers comparable to these of PBMCs'. ${ }^{216}$ Next, I tested the expression of SEAP and GLuc by detection of their enzymatic activity in cellular supernatants of THP-1 reporter cells before and during PMA-differentiation. SEAP and GLuc can be detected in the same cellular supernatant, as they utilize different substrates. As expected, SEAP enzymatic activity was at a background level in cellular supernatant of undifferentiated THP-1 cells (Fig.3-6D, 24h before PMA), as CEBPD gene displays a low basal expression in monocytes. ${ }^{62}$ PMA-differentiation activated CEBPD promoter leading to SEAP secretion during (48h with PMA) and after (48h recovery) PMA treatment (Fig.3-6D). GLuc reporter, which was expressed under control of constitutive CMV promoter, displayed its enzymatic activity in cellular supernatants of non- and PMA-differentiated THP-1 reporter cells (Fig.3-6E). Surprisingly, GLuc enzymatic activity was induced in response to PMA treatment (Fig.3-6E, 48h with PMA) and remained high after PMA treatment (Fig.3-6E, 48h recovery).

In summary, the defined CEBPD promoter as well as SEAP and GLuc reporter proteins were functional in generated THP-1 reporter cells, which have undergone the expected differentiation to $M \varphi$ in response to PMA treatment. 
A

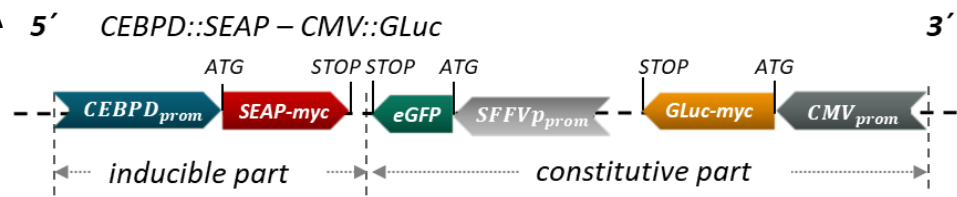

B

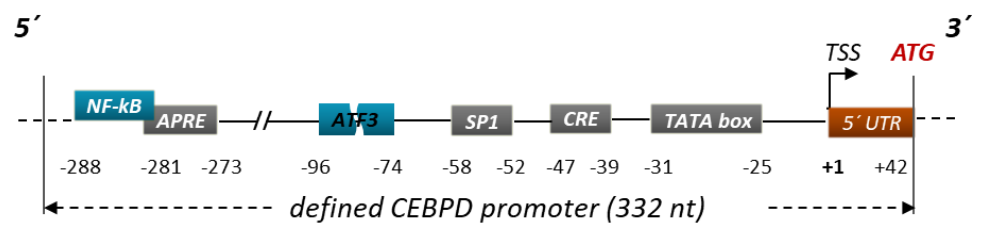

C

monocyte

(24h before PMA)

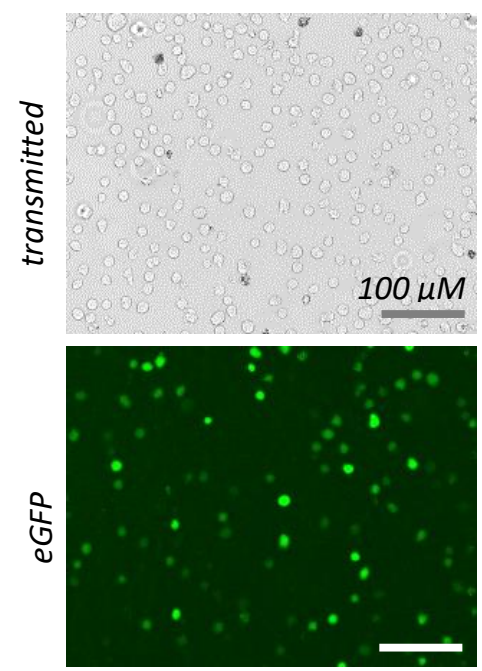

D

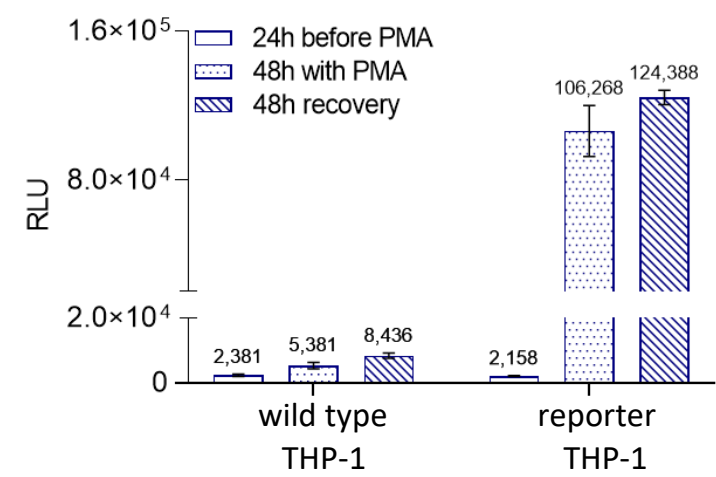

3

macrophage

(48h recovery)

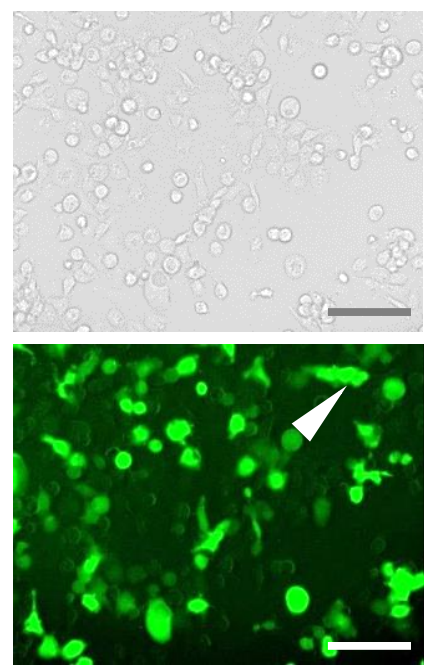

$\mathbf{E}$

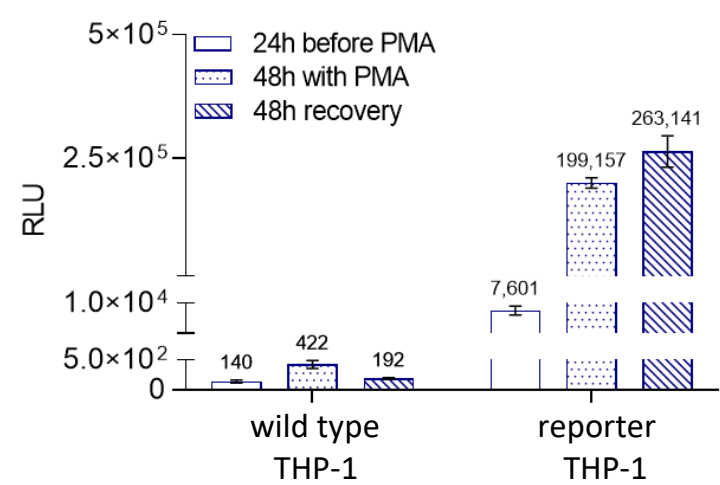

Figure 3-6: Characterization of THP-1 reporter cells stably expressing multi-gene-reporter cassette 1.0. Wild type THP-1 cells were stably transduced using a lentiviral vector encoding multi-gene-reporter cassette 1.0 (A) under control of the defined CEBPD promoter (B). THP-1 reporter cells were PMA-differentiated to $M \varphi$, following positive cell clone selection. THP-1 cells were first cultured for 24 hours in a standard cell culture medium ( $24 \mathrm{~h}$ before PMA), then treated with $50 \mathrm{ng} / \mathrm{mL}$ PMA for 48 hours ( $48 \mathrm{~h}$ with PMA), and, finally, cultured in a fresh PMA-free medium for 48 hours (48h recovery). Effect of PMA on THP-1 reporter cell morphology was examined by live-cell microscopy (C). Enzymatic activity of SEAP (D) and GLuc (E) was examined in cellular supernatants of THP-1 wild type and reporter cells at indicated time points. A: Schematic drawing of the multi-gene-reporter cassette 1.0. B: Schematic drawing of the defined CEBPD promoter containing 5'UTR, TSS, TATA box as well as known (gray boxes) and proposed (blue boxes) binding sites of regulatory TFs. Positions are indicated relative to TSS. C: Live-cell imaging of THP-1 reporter cells. During PMA-differentiation, THP-1 reporter cells became adherent and displayed heterogenic and elongated cell shape (white arrows). Scale bar: $100 \mu \mathrm{M}$. D, E: The effect of PMA on secretion of SEAP (D) and GLuc (E) in THP-1 reporter cells. Data represent mean \pm SD of three independent experiments. RLU: relative luminescence units. 


\subsubsection{Generation and functionality test of the multi-gene-reporter cassette $\mathbf{2} .0$}

\subsubsection{Modification of SEAP gene reporter: detection of ER-stress}

Integrity of the secretory pathway is an important factor that has to be considered using secreted gene reporters like SEAP. Screening compounds can potentially cause ER-stress that disturbs secretory pathway resulting in reduced SEAP secretion, which can be misinterpreted as an inhibitory effect of a screening compound on CEBPD gene expression. For a non-invasive monitoring of ER-stress during screening, I sat out to use a dual-localization mechanism that could be detected by a high content microscopy.

Secreted proteins like SEAP carry N-terminal signal peptide (SP) that is responsible for secretory pathway targeting. The SPs are short (approx. 20 amino acids) protein segments that differ in their primary amino acid sequences but display similar physio chemical properties. Further, SPs display different efficiencies according to their protein-targeting ability in response to ER-stress. To test the endogenous SEAP SP, I compared its protein targeting efficiency in absence and presence of ER-stress to this of SPs from other proteins. To do so, I modified SEAP reporter protein by its C-terminal fusion to a nuclear localization signal (NLS) and a red fluorescent protein mCherry (Fig.3-7A). Five SEAP constructs were generated under the SPs from the following proteins: SEAP, AJAP1, Prl, Prp, and one lacking the SP (Fig.3-7B). According to the proposed dual-localization mechanism, SP-SEAP-myc-NLS-mCherry reporter, which normally locates in cytosolic organelles (e.g. ER, Golgi), translocates to the nucleus in response to ER-stress (Fig.3-7C). The SP-specific differences in protein targeting efficiency in absence and presence of ER-stress I determined in DMSO- and thapsigargin-treated HEK293T reporter cells transiently expressing generated SP-SEAP-myc-NLS-mCherry proteins (Fig.3-8). Secretion of SEAP was not considerably affected by its fusion to NLS-mCherry, as detected in cellular supernatants of corresponding HEK293T reporter cells, before and after DMSO treatment (Fig.3-8A, B, C, D, grey lines) and before thapsigargin treatment (Fig.3-8A, B, C, D, green line). As expected, SP-deficient delta-SEAP-myc-NLS-mCherry protein displayed a background enzymatic activity indicating the absolute requirement of SP for protein secretion (Fig.3-8A, B, C, D, blue line). In response to the thapsigargin treatment ( $3 \mu \mathrm{M}$ for $6 \mathrm{~h}$ ), SP-SEAP-myc-NLS-mCherry secretion level was dramatically reduced in comparison to before treatment, in SP-independent manner (Fig.3-8A, B, C, D, 
A

A

$5^{\prime}$

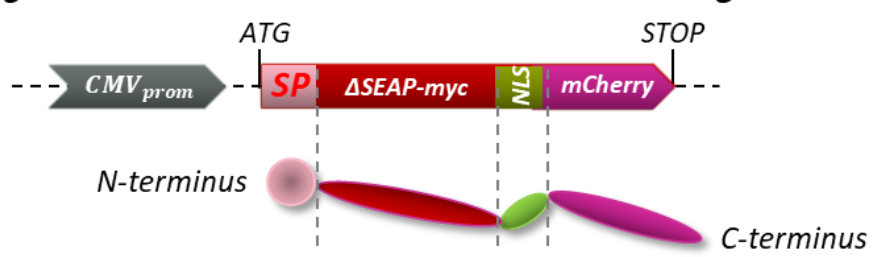

B

\begin{tabular}{l|l|l|c} 
SP & protein & sequence of signal peptide (SP) & length \\
\hline wt & endogenous signal peptide & MLLLLLLLGLRLQLSLG & 17 \\
AJAP1 & shrew-1 & MWIQQLLGLSSMSIRWPGRPLGSHAWILIAMFQLAVDLPACEA & 43 \\
Prl & prolactin & MNIKGSPWKGSLLLLLVSNLLLCQSVAP & 28 \\
Prp & prion & MANLSYWLLALFVMWWTDVGLC & 22 \\
delta, $\Delta$ & signal peptide deletion mutant & no sequence & 0 \\
\hline
\end{tabular}

C

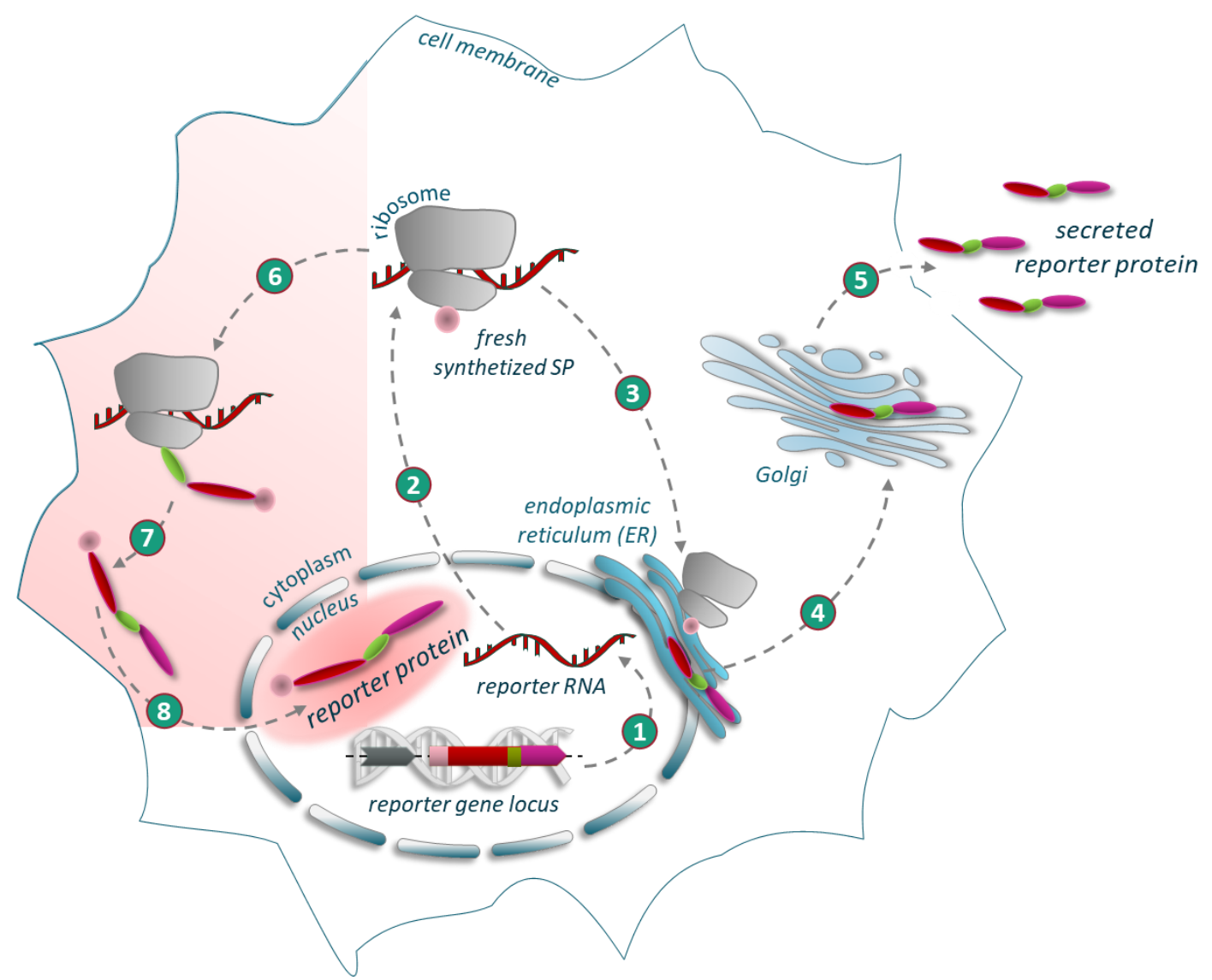

Figure 3-7: Development of SEAP-based ER-stress reporter using dual-localization mechanism. A: Schematic drawing of generated SEAP-based reporter gene and reporter protein. Here, myc-tagged SEAP (dark red) has been further modified by its C-terminally fusion to a nuclear localization signal (NLS, green) and a red fluorescent protein (mCherry, magenta). In total, five different SEAP-myc-NLS-mCherry fusion proteins were generated that carry different signal peptides (SP, pink) including wild type SEAP's SP (wt), SPs from other proteins (AJAP1, Prp, Prl), as well as delta SP (signal peptide deletion mutant). B: Primary amino acid sequences and lengths of used SPs are described in (A). C: Schematic principle of ER-stress detection using dual-localization mechanism. SP-SEAP-myc-NLS-mCherry reporter gene is transcribed into the corresponding mRNA following transfection (1). Freshly synthetized reporter mRNA translocates into the cytosol for the ribosomal translation to generate SP-SEAP-myc-NLS-mCherry protein (2). Ribosomal synthesis of proteins carrying N-terminal SP terminates in cytosol to be continued in ER-lumen. Translocated to ER (3), ribosome binds to translocon located in ER membrane (here not shown) leading to protein synthesis in ER-lumen. The fully synthetized protein is further modified through SP cleavage in ER, under- 
going protein folding and glycosylation (here not shown). Next, reporter protein is transported to Golgi (4) and later to cell membrane to be finally secreted into the supernatant (5). However, during ER-stress (red area) usual translocation process of ribosome to ER can be disturbed resulting in cytosolic protein synthesis (6). Fully synthetized proteins usually stay in cytosol being unable to enter ER and failing to be secreted (7). Here, NLS-carrying reporter proteins are recognized by other protein machinery and targeted to the nucleus (8). ER-stress, potentially caused by a screening compound during HTS, may be detected through a monitoring of SEAP-myc-NLS-mCherry localization by high-content microscopy.

red line). The remaining SEAP enzymatic activity, which was normalized to the corresponding values before treatment and compared to that of DMSO-treated control (set as 1), was between 18 to 27 per cent in response to the thapsigargin-induced ER-stress (Fig.3-8E). Tested SPs displayed no significant differences in their individual ER-stress sensitivity. Elevated protein level of GRP78 - a well-established ER-stress marker ${ }^{217}$ - confirmed ER-stress onset in thapsigargin-treated samples, as demonstrated by Western Blotting (Fig.3-8F). Fluorescent microscopy analysis showed that the red fluorescence ability of mCherry was unaffected by its fusion to SEAP-myc-NLS and could be detected in fixed HEK293T reporter cells (Fig.3-8G). As expected, delta-SEAP-myc-NLS-mCherry protein located exclusively in the nucleus, unable to be targeted to ER (Fig.3-8G, last panel). Different extend of the nuclear reporter localization characterized dissimilar protein targeting efficiencies of tested SPs in the absence of ER-stress. Wild type SEAP and AJAP1 SPs were less efficient in comparison to Prl and Prp SPs (Fig.3-8G, first panel), indicated by the absent non-nuclear localization of corresponding reporter proteins. Prl SP showed the highest SP efficiency compared to other SPs tested exhibiting hardly any nuclear localization. Further, Prl-SEAP-myc-NLS-mCherry translocated to the nucleus in response to the thapsigargin treatment (Fig.3-8G, middle panel), visually indicating the onset of ER-stress. These preliminary data characterized PrI-SEAP-myc-NLS-mCherry as a potential ER-stress reporter.

\subsubsection{Multi-gene-reporter cassette 2.0 is functional in HEK293T cells}

The modified Prl-SEAP-myc-NLS-mCherry protein I combined with GLuc-myc and copepod GFP (copGFP) to a multi-gene-reporter cassette 2.0. Each of the used reporter proteins displayed its purpose-dependent function in the cassette 2.0 (Fig.3-9A). I further modified copGFP by its C-terminal fusion to NLS, as visual detection of ER-stress can be supported by the optical marking of the nucleus. A predominantly non-nuclear protein 
A

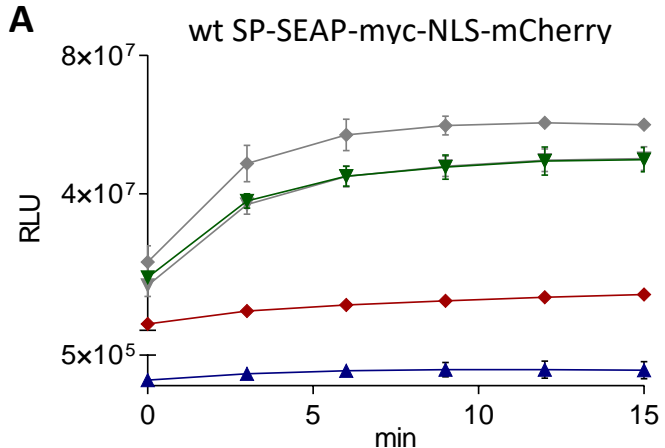

C

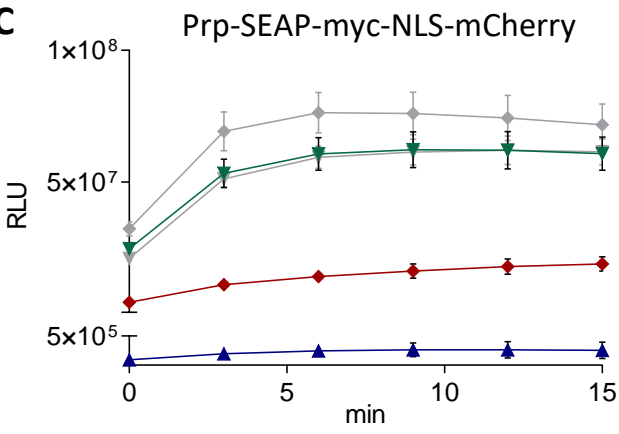

B

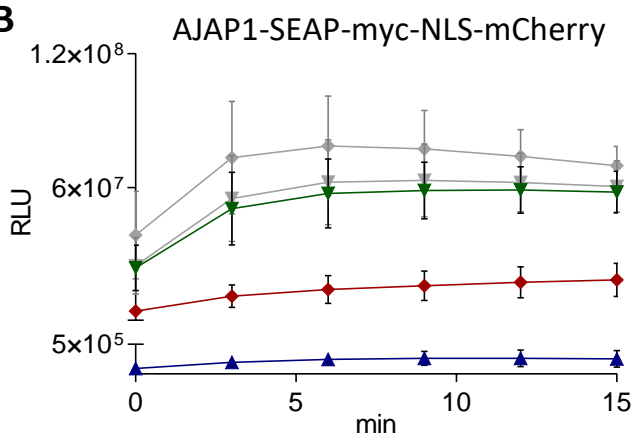

D
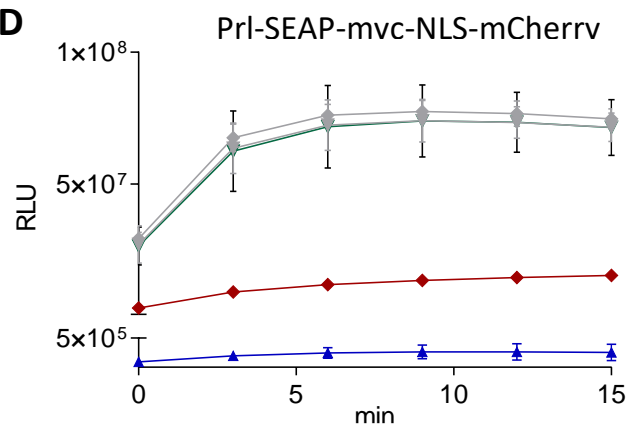

$\neg$ thapsigargin, before treatment $\neg-$ thapsigargin, after treatment $\neg$ delta SEAP-myc $\rightarrow$ DMSO, bevore treatment $\diamond-$ DMSO, after treatment

E

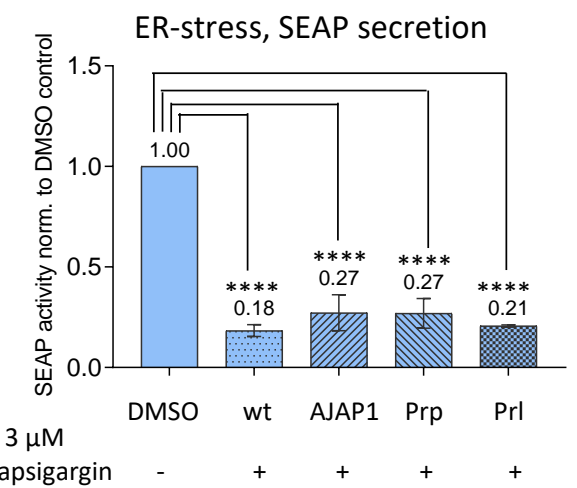

$\mathbf{F}$

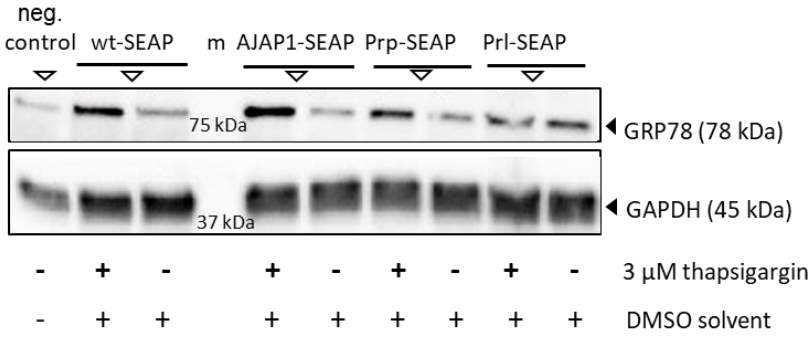

Figure 3-8: Characterization of SP-dependent ER-stress sensitivity and protein targeting efficiency. Wild type HEK293T cells were cultured on object slides in a 6-well format for 24 hours followed by transient transfection with wt-, AJAP1-, Prp- or Prl-SEAP-myc-NLS-mCherry-expressing plasmids. Cellular supernatants were collected next day after transfection (condition "before treatment"). Next, HEK293T reporter cells were treated with medium supplemented with either $3 \mu \mathrm{M}$ thapsigargin or equal volume of DMSO solvent control for $6 \mathrm{~h}$ (condition "after treatment"). Changes in SEAP secretion were detected in corresponding cellular supernatants (A-E). Cells attached to object slides were used for microscopic analysis (G) and the remaining cells were analyzed by Western Blotting (F). A, B, C, D: Chemiluminescent SEAP assay. Enzymatic activity of SEAP-myc-NLS-mCherry was monitored in cellular supernatants of HEK293T reporter cells, before and after DMSO or thapsigargin treatment. Background enzymatic activity was detected in cellular supernatant of HEK293T reporter cells transiently expressing delta-SEAP-myc-NLS-mCherry. Each point represents an average signal \pm SD of three independent experiments. E: ER-stress-mediated reduction in SEAP secretion. Enzymatic signals of thapsigargin treated samples, detected 15 min after substrate addition, were normalized to their corresponding DMSO control (summarized in one bar, which is set as 1). Statistical analysis was done via ordinary one-way ANOVA with Dunnett's multiple comparisons test, with a single pooled variance. Data represent mean \pm SD of three independent experiments. F: Western Blotting analysis of protein extracts from non-treated wild type HEK293T (neg. control) and thapsigargin or DMSO treated HEK293T reporter cells. GRP78 (ER-stress marker) and GAPDH (loading control) proteins were detected using anti-GRP78 (1:1000, Santa Cruz Biotechnology) and anti-GAPDH (1:1000, Cell Signaling Technology) primary antibodies, respectively. Here, HRP-conjugated secondary antibodies were used. Representative result of three independent experiments is shown. $\mathrm{m}$ : protein maker; $* * * * \mathrm{p}$-value $<0.0001$ 
Figure3-8: Continuance.

G

SP-SEAP-myc-NLS-mCherry, DMSO solvent control

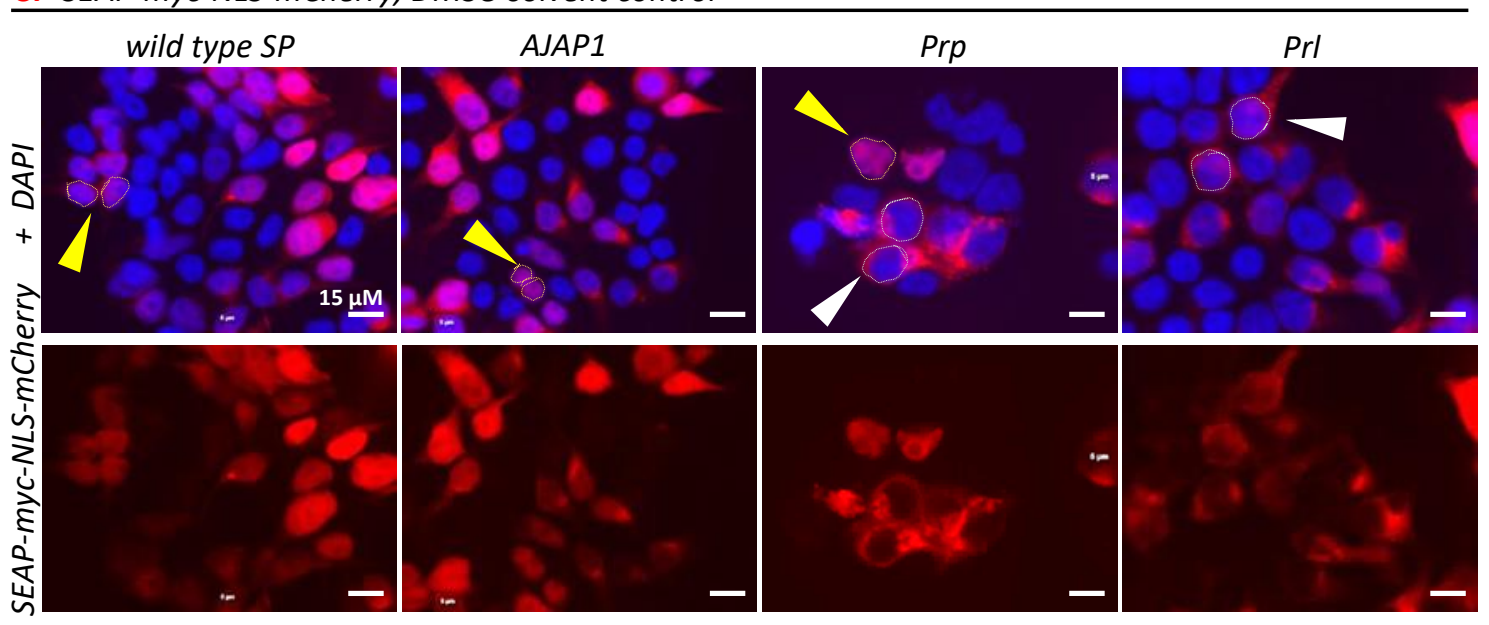

SP-SEAP-myc-NLS-mCherry, $3 \mu M$ thapsigargin treatment

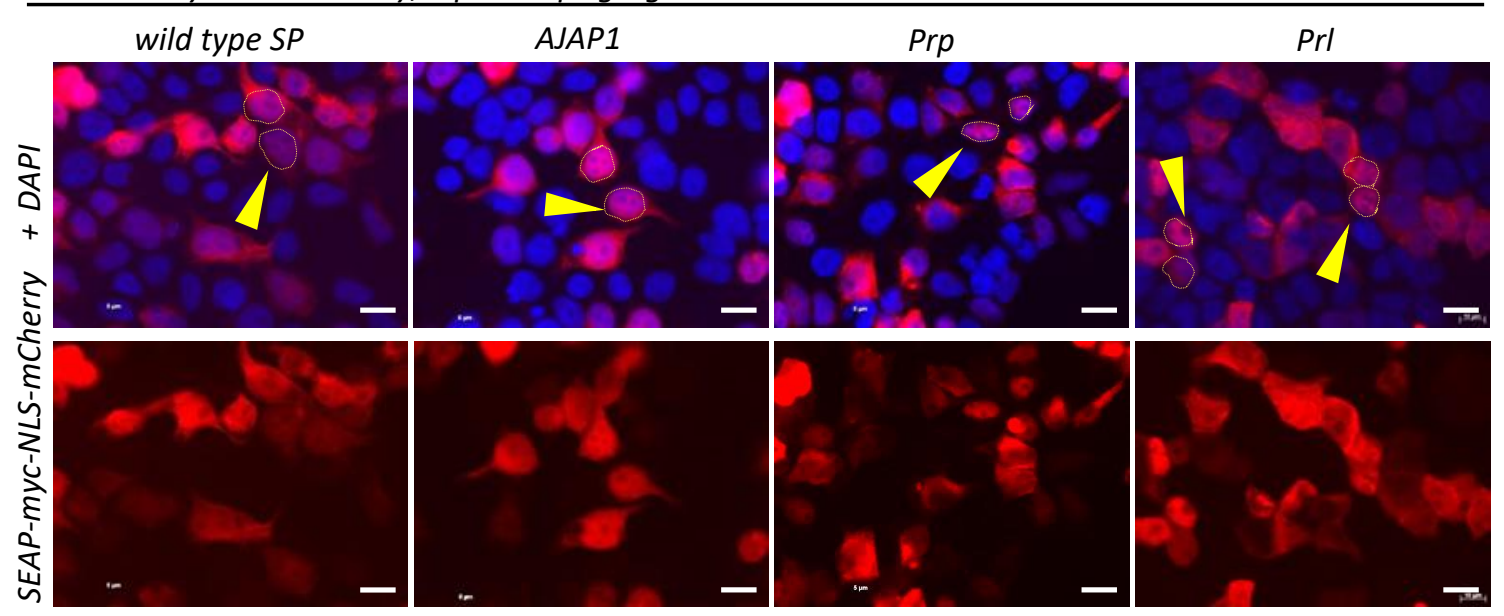

$\triangle S P-S E A P-m y c-N L S-m C h e r r y, D M S O$ solvent control

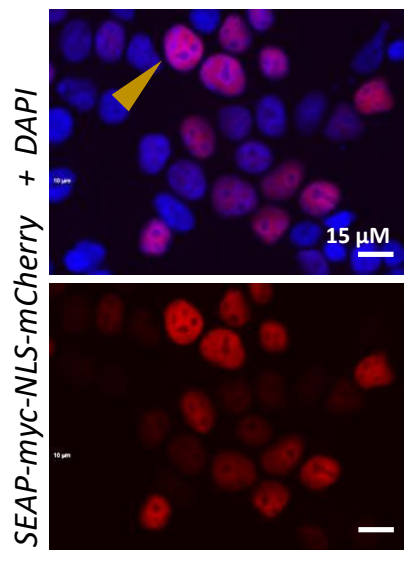

Figure 3-8: Characterization of SP-dependent ER-stress sensitivity and protein targeting efficiency. G: Microscopic analysis of SP-dependent changes in reporter protein localization in response to thapsigargin treatment. Following DMSO or $3 \mu \mathrm{M}$ thapsigargin treatment for $6 \mathrm{~h}$, HEK293T cells transiently expressing SP-SEAP-myc-NLS-mCherry reporter proteins were fixed, permeabilized, and stained with DAPI nuclear staining (blue). After staining, cellular localization of mCherry-containing reporter protein (red, $\lambda_{\max } 610 \mathrm{~nm}$ ) was detected via fluorescence microscopy. Lacking SP, delta-SEAP-myc-NLS-mCherry ( $\triangle S P$ ) exclusively localizes in the nucleus (orange arrow). The ex-nuclear (white arrows) or nuclear (yellow arrows) localization of SP-containing reporter proteins is indicated. Representative pictures of three independent experiments are shown. Scale bar: $15 \mu \mathrm{M}$.

localization of Prl-SEAP-myc-NLS-mCherry and an exclusively nuclear protein localization of copGFP reporter confirmed the functionality of the generated cassette (Fig.3-9B), which was transiently expressed in HEK293T cells under control of the defined CEBPD promoter. These preliminary data showed a potential use of the multi-gene-reporter 
cassette 2.0 during screening for the non-invasive monitoring of CEBPD target promoter activity and of ER-stress onset.

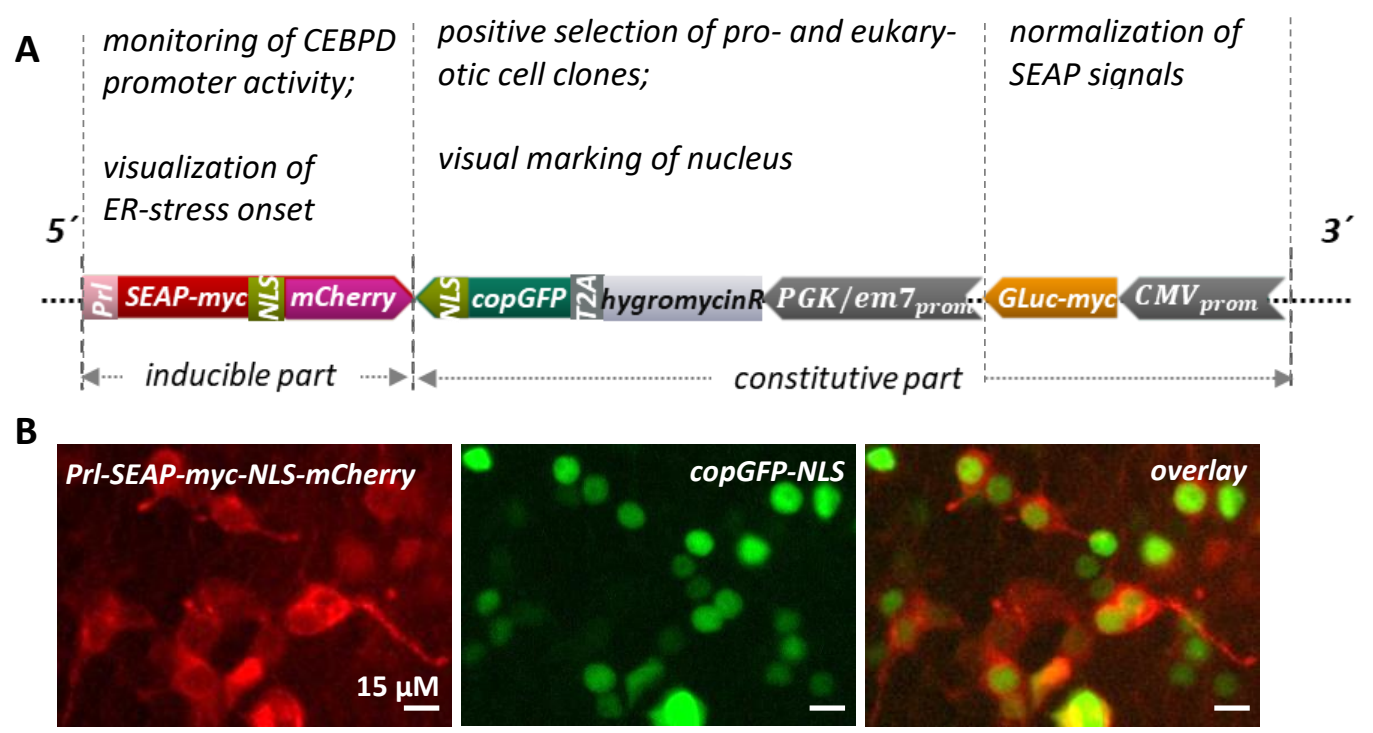

Figure 3-9: Functionality test of multi-gene-reporter cassette 2.0 in HEK293T cells. A: Schematic drawing of the structure and corresponding functions of the multi-gene-reporter cassette 2.0. B: Live-cell imaging of HEK293T cells transiently expressing the described 2.0 cassette (A). Here the cassette is expressed under control of the defined CEBPD promoter (Fig.3-6B), in a pcDNA3.1(-) backbone. Prl-SEAP-myc-NLSmCherry (red) displayed predominantly non-nuclear and copGFP-NLS (green) showed exclusively nuclear protein localizations in the absence or ER-stress. Scale bar: $15 \mu \mathrm{M}$.

\subsubsection{Validation of the cellular system and specification of the screening procedure}

\subsubsection{M1-polarized THP-1 reporter $\mathrm{M} \varphi$ serve as a reliable cellular system}

I validated the generated THP-1 reporter cell line according to its suitability and reliability for the screening. To do so, I polarized PMA-differentiated THP-1 reporter M $\varphi$ toward the pro-inflammatory M1 state by the treatment with LPS and IFN-g (M1 treatment). THP-1 reporter M $\varphi$ changed their cellular morphology like wild type THP-1 M $\varphi$ from a round to a spindle-like in response to the M1 treatment (Fig.3-10A).

Further, I tested transcription activation of the endogenous CEBPD gene in response to LPS treatment in wild type non- and PMA-differentiated THP-1 cells (Fig.3-10B). The LPSmediated CEBPD induction is reported to be rapid and transient, ${ }^{36}$ with a maximal mRNA expression level at 4 hours after LPS exposure in murine M $\varphi .{ }^{71}$ LPS treatment (100 pg/mL-0.1 $\mu \mathrm{g} / \mathrm{mL}$ ) failed to activate the endogenous CEBPD in non-differentiated wild type THP-1 cells (Fig.3-10B, 6h, 24h). However, CEBPD gene expression was significantly activated in PMA-differentiated THP-1 M $\varphi$ in response to 50 and $100 \mathrm{ng} / \mathrm{mL}$ LPS 
relative to solvent control, 6 hours after treatment (Fig.3-10B, 6h (PMA)). Treatment with $20 \mathrm{ng} / \mathrm{mL}$ IFN-g and 0.1 or $1 \mu \mathrm{g} / \mathrm{mL}$ LPS also significantly activated gene expression of endogenous CEBPD in PMA-differentiated THP-1 reporter $M \varphi, 6$ hours post-treatment (Fig.3-10C). Gene expression pattern of SEAP reporter expressed under control of the defined CEBPD promoter (CEBPD::SEAP) mirrored that of the endogenous CEBPD (Fig.3-10D) in M1-polarized THP-1 reporter M $\varphi$. Further, elevated SEAP secretion indicated activation of CEBPD::SEAP gene expression in M1-polarized THP-1 reporter M $\varphi$ (Fig.3-10E). However, changes in SEAP secretion level could be first reliably detected at a later time point (24 hours). Such a shift in SEAP detection in cellular supernatant may be caused by: i) time needed for protein synthesis and secretion; ii) relatively weak promoter strength of CEBPD causing a slow accumulation of SEAP upon promoter activation.

In summary, in M1-polarized THP-1 reporter M $\mathrm{M}$ : i) mRNA expression of endogenous CEBPD was induced; ii) mRNA expression pattern of the reporter CEBPD::SEAP mirrored that of the endogenous CEBPD; iii) SEAP reporter reliably indicated activation of the defined CEBPD promoter in the corresponding cellular supernatant. The M1-polarized THP-1 reporter $\mathrm{M} \varphi$ represented, therefore, a suitable and reliable cellular system for the screening.

\subsubsection{Specification of the final screening procedure}

Following conditions needed to be specified for the final screening protocol: i) cell culture and differentiation formats; ii) M1 treatment condition and timing; iii) control substance identity; iv) normalization assay; v) down-scaling of SEAP readout assay.

During screening, it was important to use fresh one-week THP-1 reporter cells to prevent possible CEBPD promoter methylation-mediated reduction in SEAP secretion level. The ongoing methylation of the GC-reach CEBPD promoter sequence may silence its activity resulting in reduced SEAP secretion. SEAP enzymatic activity was more than two times lower in cellular supernatants of five-week old THP-1 reporter $\mathrm{M} \varphi$ in comparison to this of one-week old THP-1 reporter $M \varphi$ (chapter 8.4.1, Fig.8-7). 

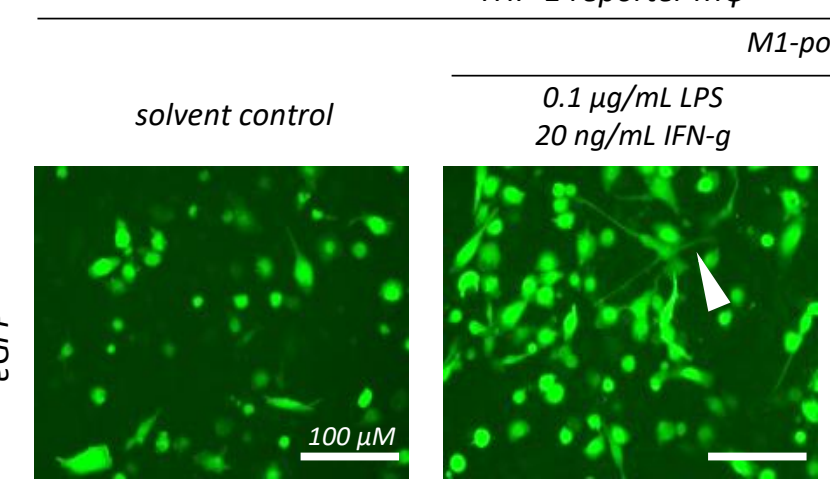

B

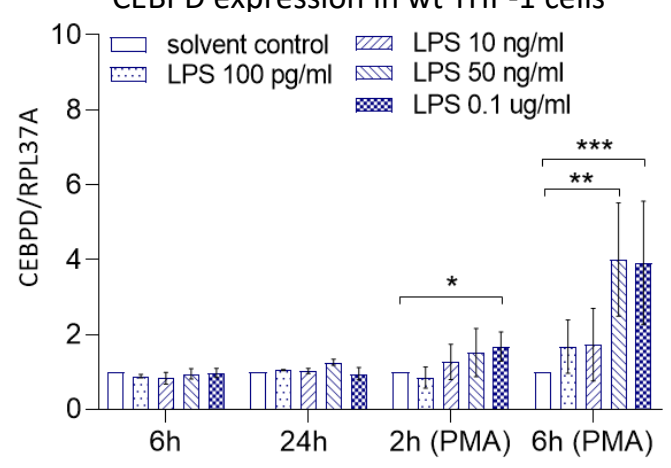

D CEBPD::SEAP expression in THP-1 reporter $M \varphi$

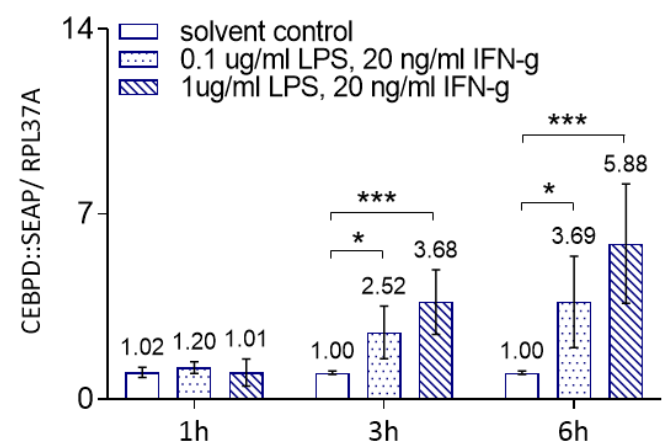

C

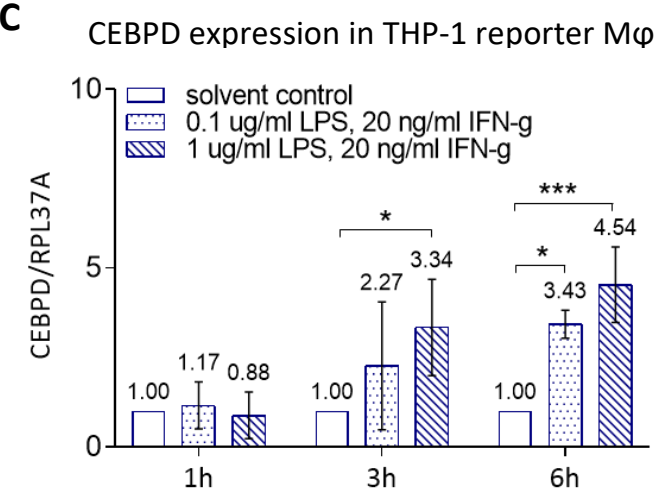

$\mathbf{E}$ SEAP assay, THP-1 reporter M $\varphi$

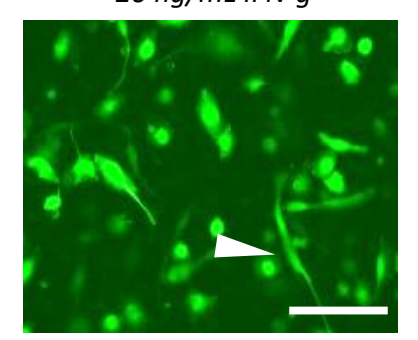
$1 \mu \mathrm{g} / \mathrm{mL} L P S$
$20 \mathrm{ng} / \mathrm{mL}$ IFN-g

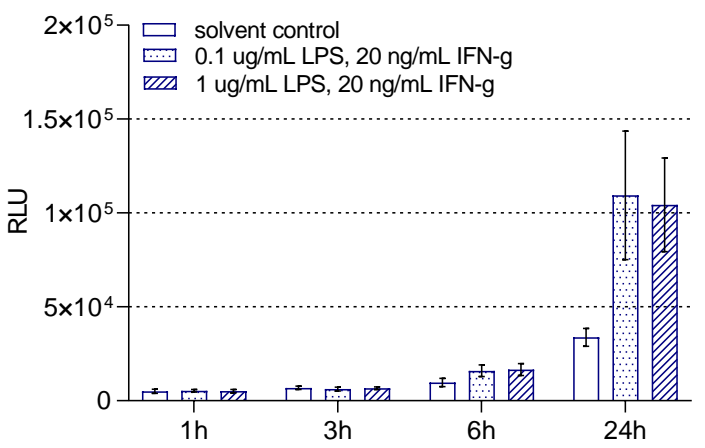

Figure 3-10: Characterization of SEAP as a reliable gene reporter for monitoring of CEBPD promoter activity in THP-1 reporter M $\varphi$. PMA-differentiated THP-1 reporter $\mathrm{M} \varphi$ stably expressing multi-gene-reporter cassette $1.0(A, C, D, E)$ were polarized toward the M1 state through the treatment with LPS $(0.1$ or $1 \mu \mathrm{g} / \mathrm{mL})$ and IFN-g $(20 \mathrm{ng} / \mathrm{mL})$ for up to 24 hours. Relative gene expression in cell lysates (B, C, D) and enzymatic SEAP reporter activity in corresponding cellular supernatants (E) were determined. Solvent control was represented by THP-1 cells treated with PBS for indicated time points. A: Live-cell imaging of PMA-differentiated, non-polarized (solvent control, PBS) and M1-polarized THP-1 reporter M $\varphi$. M1-polarized reporter $M \varphi$ changed their morphology displaying spindle-like cell shape, $24 \mathrm{~h}$ after LPS- and IFN-g treatment (white arrows). Scale bar: $100 \mu \mathrm{M}$. B: Endogenous CEBPD gene expression analysis in nondifferentiated and PMA-differentiated (PMA) wild type THP-1 cells in response to LPS treatment $(100 \mathrm{pg} / \mathrm{mL}-0.1 \mu \mathrm{g} / \mathrm{mL})$ for 2, 6, and 24h. C, D: Endogenous CEBPD (C) and reporter CEBPD::SEAP (D) gene expression analysis in THP-1 reporter $M \varphi$ in response to LPS- and IFN-g treatment for 1,3 , or $6 \mathrm{~h}$. RPL37A housekeeping gene was used for normalization (RT-qPCR, $\triangle \Delta C_{t}$ method). Differences in CEBPD gene expression levels were analyzed relative to solvent control via one-way ANOVA or Kruskal-Wallis test with Dunn's correction for multiple comparisons. Data represent mean \pm SD of three independent experiments. $* p<0.05 ; * * p<0.005 ; * * * p<0.001$. E: Chemiluminescent SEAP assay. SEAP secretion level was monitored in response to the treatment with PBS- or LPS- and IFN-g for 1, 3, 6, or 24h, in cellular supernatants of THP-1 reporter $\mathrm{M} \varphi, 40$ minutes after substrate addition. Data represent mean \pm SD of three independent experiments. 
THP-1 reporter cells were PMA-differentiated to $M \varphi$. Differentiation of THP-1 reporter $M \varphi$ directly on a plate, in 384 individual wells, may result in well-to-well differences in differentiation state due to their high cellular plasticity. Such variations can negatively impact readout reliability, as activation of CEBPD promoter is differentiation-dependent (Fig.3-6D). Therefore, THP-1 reporter cells were PMA-differentiated in a bulk format, in large-volume cell culture flasks, and then seeded on 384-well plates (Fig.3-11), to ensure their equal differentiation state.

The M1-polarization of THP-1 reporter M $\varphi$ was a necessary condition for the assay. Treatments with both LPS as $0.1 \mu \mathrm{g} / \mathrm{mL}$ or $1 \mu \mathrm{g} / \mathrm{mL}$ and $20 \mathrm{ng} / \mathrm{mL}$ IFN-g significantly upregulated CEBPD gene expression (Fig.3-10C). Assuming LPS-mediated effect sizes de-

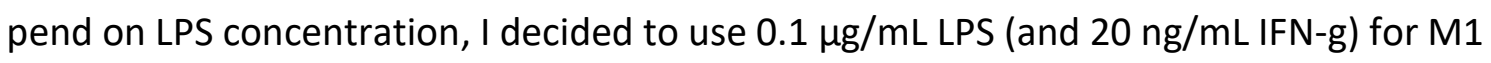
treatment, as potential CEBPD-inhibitory compounds could rather show their effects then a lower LPS amount is used. Pre-treatment of THP-1 reporter $M \varphi$ with screening compounds prior to M1 treatment could further increase the probability of CEBPD-inhibitory compound identification (Fig.3-11).

During screening, control substances act as a benchmark for screening assay quality. Among the tested substances including rosmanol, inotilone, lovastatin, and p38 MAP kinase inhibitor IV, only trichostatin A (TSA) displayed a noticeable effect. TSA reliably upregulated SEAP secretion in M1-polarized THP-1 reporter $\mathrm{M} \varphi$ (data not shown) and served, therefore, as a control substance during screening.

Unexpectedly, GLuc showed changes in gene expression during experimental testing of control substances (data not shown). GLuc assay was, therefore, not suitable for normalization and was replaced by the CellTiter-Glo ${ }^{\circledR}$ assay (CTG).

To enable a semi-automatic screening performance and to reduce screening costs, I down-scaled SEAP readout assay to the 384-well format. Chemiluminescent SEAP assay was demonstrated to be sufficiently robust and sensitive for small sample volumes (chapter 8.4.2, Fig.8-8). 


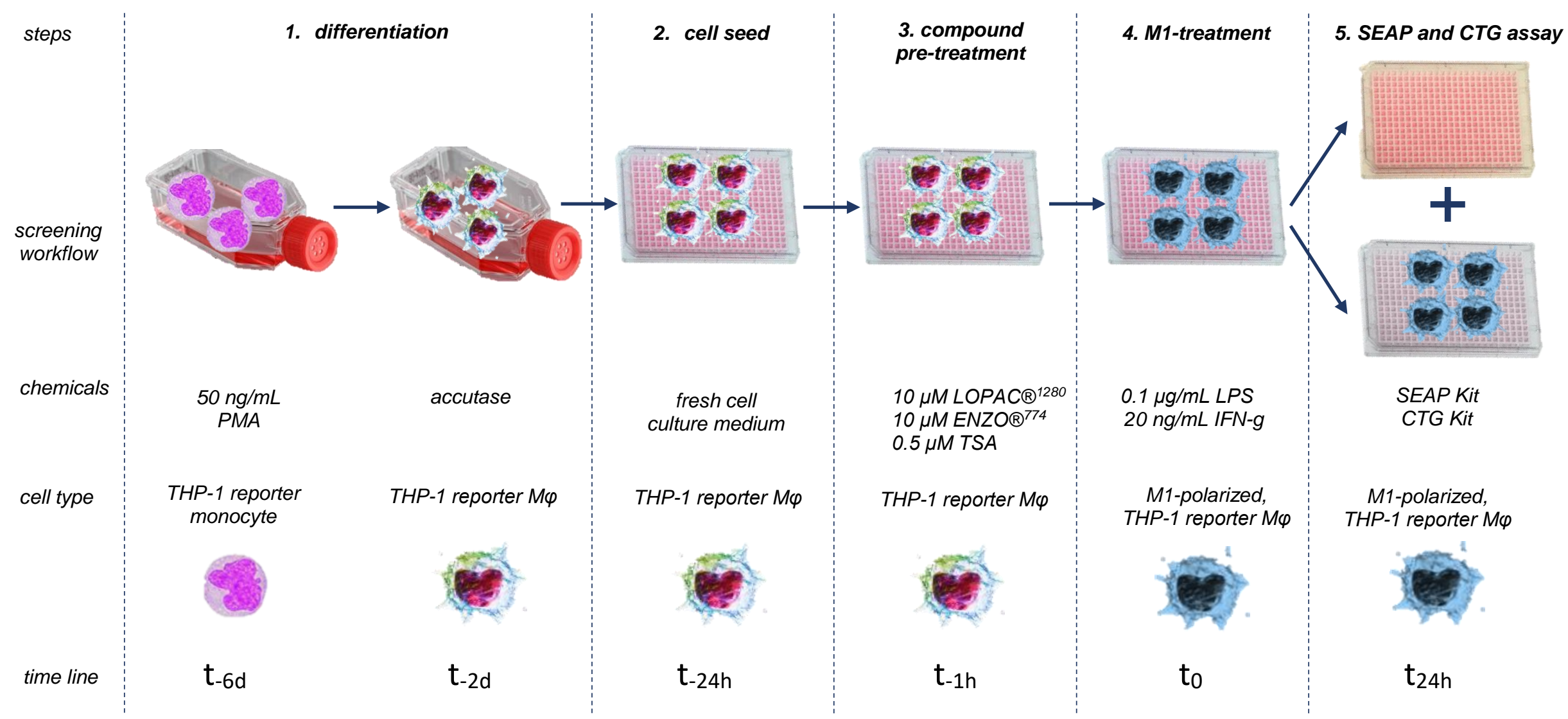

Figure 3-11: Schematic workflow of the screening. THP-1 reporter cells were PMA-differentiated in a bulk format in T175 cell culture flasks (t-6d) for 48 hours and cultured in fresh PMA-free cell culture medium for 72 hours ( $t$-2d). Next, PMA-differentiated THP-1 reporter M $\varphi$ were seeded in 384-well format with 10,000 cell per well ( $\mathrm{t}$-24h). Next day, the cellular supernatants were robotically removed and $20 \mu \mathrm{L}$ of fresh cell culture medium were added manually to 384-well plates. THP-1 reporter M $\varphi$ were pre-treated with compounds from LOPAC ${ }^{\oplus 1280}$ and ENZO ${ }^{\circledR 774}$ libraries (final concentration $10 \mu \mathrm{M}$ ) as well as with TSA control compound (final concentration $0.5 \mu \mathrm{M}$ ) for 1 hour (t-1h).

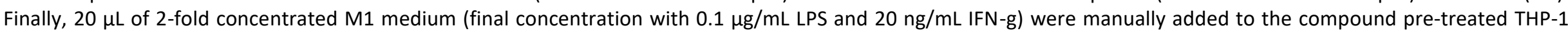
reporter $M \varphi\left(t_{0}\right)$. After M1-treatment, the final compound concentration was restored. Next day, cell culture supernatants were collected for the chemiluminescent SEAP assay (assay readout). Cell viability was estimated by CellTiter-Glo ${ }^{\circledR}$ assay (CTG) ( $\left.\mathrm{t}_{24 \mathrm{~h}}\right)$. 


\subsection{Screening for CEBPD-modulating compounds}

\subsubsection{High-throughput screening of LOPAC ${ }^{\circledR 1280}$ and ENZO ${ }^{\circledR 774}$ libraries}

We screened LOPAC ${ }^{\circledR 1280}$ and ENZO ${ }^{\circledR 774}$ compound libraries, which contained 1280 pharmacological active and 774 approved therapeutic compounds, respectively. Each screening plate contained following conditions: MO control (PMA-differentiated, nonpolarized, non-treated THP-1 reporter $\mathrm{M} \varphi$ ), M1 solvent control (PMA-differentiated, M1-polarized, DMSO pre-treated THP-1 reporter $M \varphi$ ), M1 TSA control (PMA-differentiated, M1-polarized, TSA pre-treated THP-1 reporter $M \varphi$ ), and $\mathrm{M} 1$ compound treatment (PMA-differentiated, M1-polarized, compound pre-treated THP-1 reporter M $\varphi$ ). We performed screening twice (read 1 and read 2), testing repeatability and enhancing reliability. For each of the four LOPAC $^{\circledR 1280}$ (Fig.3-12, chapter 8.6.1, Fig.8-11) and three ENZO $^{\circledR 774}$ (Fig.3-13, chapter 8.6.1, Fig.8-12) screening plates, I displayed enzymatic SEAP signals from read 1 in correlation to the corresponding signals from read 2. As expected, SEAP secretion level in M1 solvent control condition (Fig.3-12, Fig.3-13, red dots) was increased in comparison to the background SEAP secretion level in MO control condition (Fig.3-12, Fig.3-13, green dots). SEAP secretion level was elevated in response to TSA treatment relative to that in M1 solvent control condition (Fig.3-12, Fig.3-13, blue dots), as already observed during screening development (data not shown). Each compoundcaused SEAP signal (Fig.3-12, Fig.3-13, gray dots) I characterized according to its difference to an average signal of the corresponding M1 solvent control (Fig.3-12, Fig.3-13, red lines). First, I calculated means ( $\mathrm{n}$ ) and standard deviations (SD) of SEAP signals from 16 wells of the M1 solvent control condition, for each read and plate and applied a scoring matrix by adding (Fig.3-12, Fig.3-13, black lines) and subtracting (Fig.3-12, Fig.3-13, green lines) up to three-fold SDs from the corresponding average signal of M1 solvent control condition. According to a "three-sigma rule of thumb", about $99.7 \%$ of values lie within the three standard deviations of the mean. ${ }^{218}$ Therefore, a value deviating further than three SDs can be considered as a hit. For the obtained screening data, this assumption becomes more robust if the individual value difference was detected in both reads. Each of the screening compounds, I ranked according to the strength and the reproducibility of the corresponding SEAP signal. The best ranking score " $A$ " indicates a compound deviating over three SDs in both reads. (Fig.3-14). 


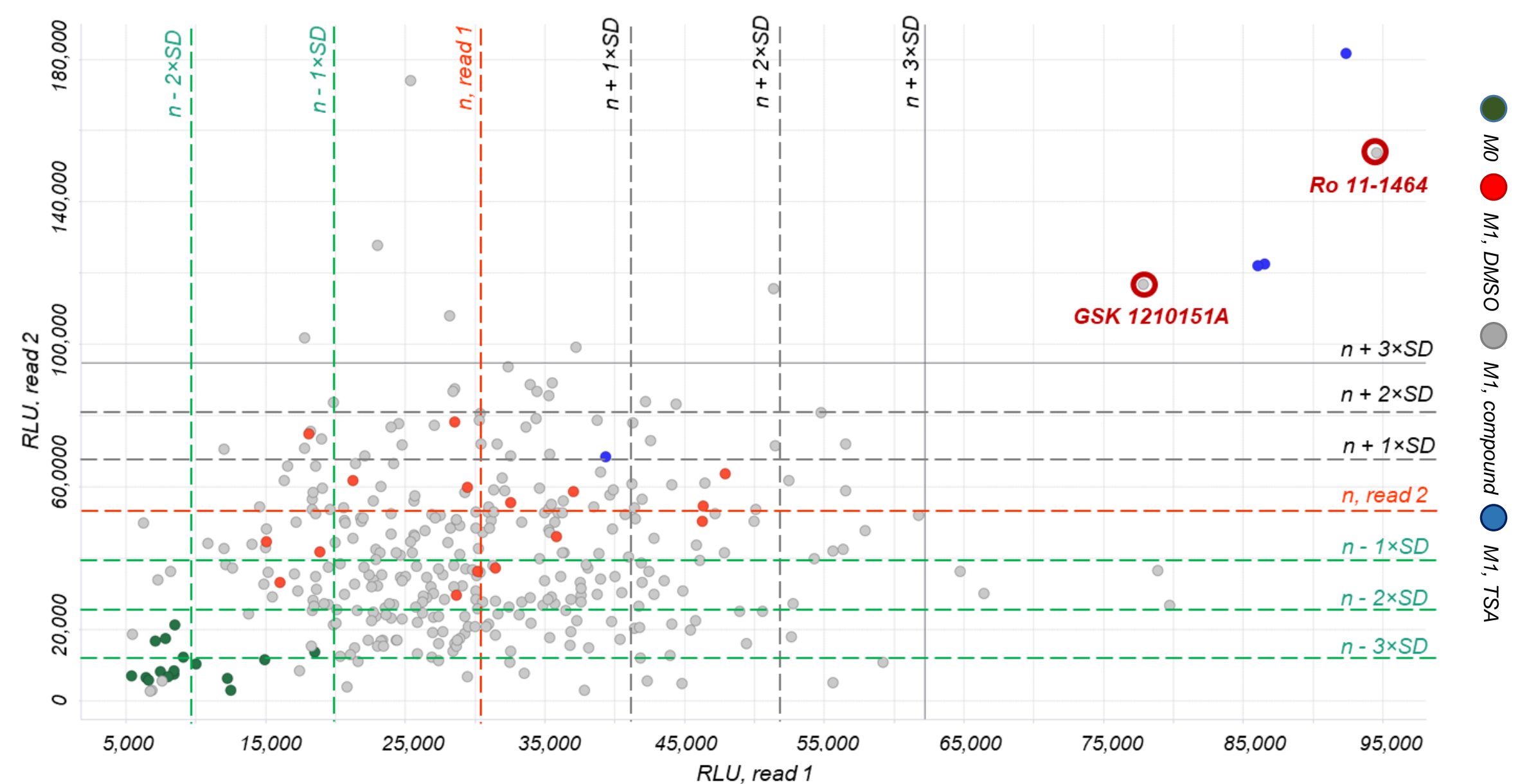

Figure 3-12: Identification of hit compounds derived from screening of LOPAC ${ }^{\oplus 1280}$ library. Screening of LOPAC ${ }^{\oplus 1280}$ library included four 384 -well plates, one of which is shown here. Please refer to chapter 8.6.1 Fig.8-11 for the remaining plates. Enzymatic SEAP signals were detected in cellular supernatants of non-polarized and non-treated (M0 control; green dots) and M1-polarized either with DMSO (M1, DMSO; red dots), compound (M1, compound; gray dots), or TSA (M1, TSA; blue dots) pre-treated THP-1 reporter $\mathrm{M} \varphi$. SEAP signals from read 1 were plotted on $\mathrm{X}$-axis and corresponding signals from read 2 on $\mathrm{Y}$-axis. Compounds showing strong activatory or inhibitory effect on SEAP secretion were identified in areas over ( $n+1 / 2 / 3 \times S D$, black line) or under $(n-1 / 2 \times S D$, green line) up to three SDs of the mean signal ( $n$, read line), calculated for the "M1, DMSO" condition (solvent control), respectively. Selected compounds (red circles) are summarized in Table 3.3. 


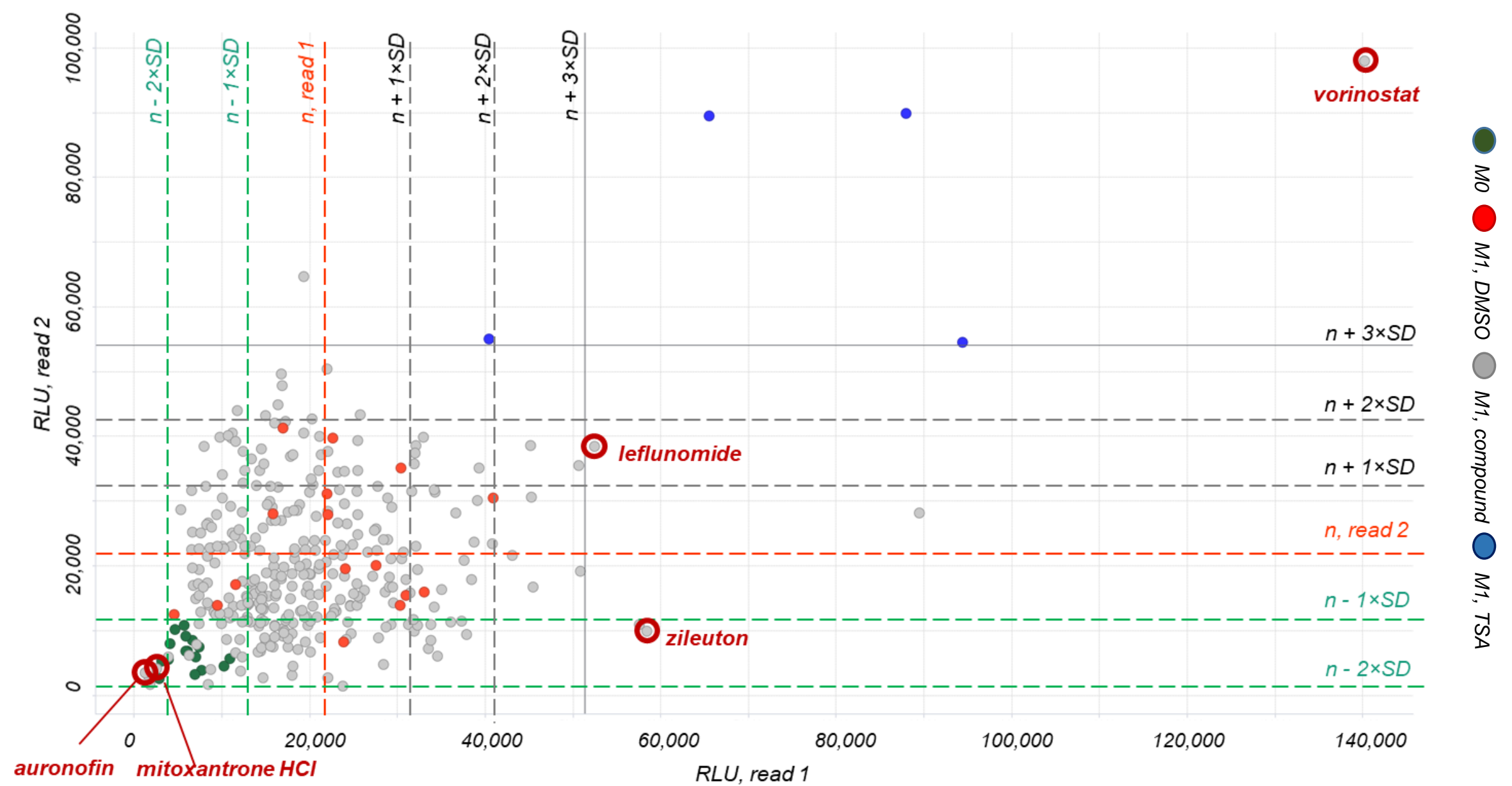

Figure 3-13: Identification of hit compounds derived from screening of ENZO ${ }^{\oplus 774}$ library. Screening of ENZO ${ }^{\oplus 74}$ library included three 384 -well plates, one of which is shown here. Please refer to chapter 8.6.1 Fig.8-12 for the remaining plates. Enzymatic SEAP signals were detected in cellular supernatants of non-polarized and non-treated (M0 control; green dots) and M1-polarized either with DMSO (M1, DMSO; red dots), compound (M1, compound; gray dots), or TSA (M1, TSA; blue dots) pre-treated THP-1 reporter $\mathrm{M} \varphi$. SEAP signals from read 1 were plotted on $\mathrm{X}$-axis and corresponding signals from read 2 on $\mathrm{Y}$-axis. Compounds showing strong activatory or inhibitory effect on SEAP secretion were identified in areas over ( $n+1 / 2 / 3 \times S D$, black line) or under $(n-1 / 2 \times S D$, green line) up to three SDs of the mean signal ( $n$, read line), calculated for the "M1, DMSO" condition (solvent control), respectively. Selected compounds (red circles) are summarized in Table 3.3. 


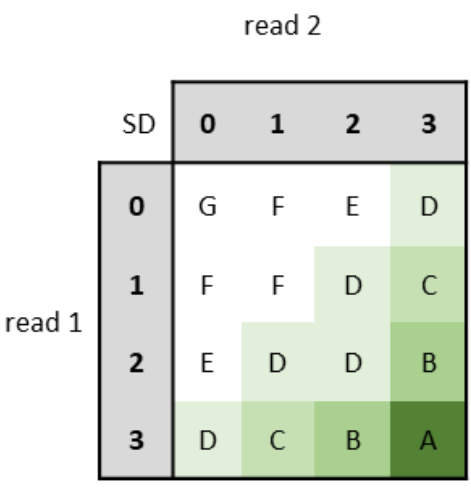

Figure 3-14: Scoring matrix used to set the compound ranking. Each of the screening compounds (2054 in total) obtained its individual ranking score (from A-the best to G-the worst) considering the strength and the reproducibility of the corresponding SEAP signal.

Following the ranking, I defined screening compounds according to their effect on the SEAP signal relative to the M1 solvent control: compounds with a high SEAP signal as "activatory-acting" and compounds with a low SEAP signal as "inhibitory-acting". I summarized the ranked screening compounds from LOPAC ${ }^{\circledR 1280}$ (Table 3.1) and ENZO ${ }^{\circledR 774}$ (Table 3.2) libraries according to their activatory or inhibitory activity and the obtained ranking score.

After classification, I selected the most prominent compounds considering both their rankling score and the corresponding pharmacological effect. Whereas A-compounds (GSK 1210151A, Ro 11-1464, and vorinostat) I automatically considered as hits, B-, C-, and D-ranked activatory-acting compounds I first evaluated according to their pharmacological action. From the LOPAC ${ }^{\otimes 1280}$ library (chapter 8.5, Fig.8-9), I selected compounds that display an anti-inflammatory effect (13-cis-retinoic acid and bropirimine). From ENZO ${ }^{\circledR 74}$ library (chapter 8.5, Fig.8-10), I selected compounds that are used for the treatment of inflammatory diseases like rheumatoid arthritis (leflunomide) and asthma (zileuton). As the inhibitory-acting compounds from both libraries displayed rather low ranking score (D-F), I selected hit compounds regarding to their pharmacological action and intensity of the corresponding SEAP signal. From LOPAC ${ }^{\circledR 1280}$ library, I chose compounds that display the most prominent difference in SEAP signal intensity relative to this of $\mathrm{M} 1$ solvent control condition (lasofoxifene tartrate and sanguinarine chloride) or modulate relevant pharmacological pathways (roscovitine). From the ENZO ${ }^{\circledR 774}$ library, I chose compounds that act anti-inflammatory (auranofin, ketorolac tromethamine, fluocinonide, amcinonide, and halcinonide) or immune-suppressive (mitoxantrone). In total, I identified 18 compounds: nine with a high and nine with a low SEAP signal (Table 3.3). 
Table 3.1: The LOPAC $^{\circledR 1280}$ library compounds sorted according to their rankig score.

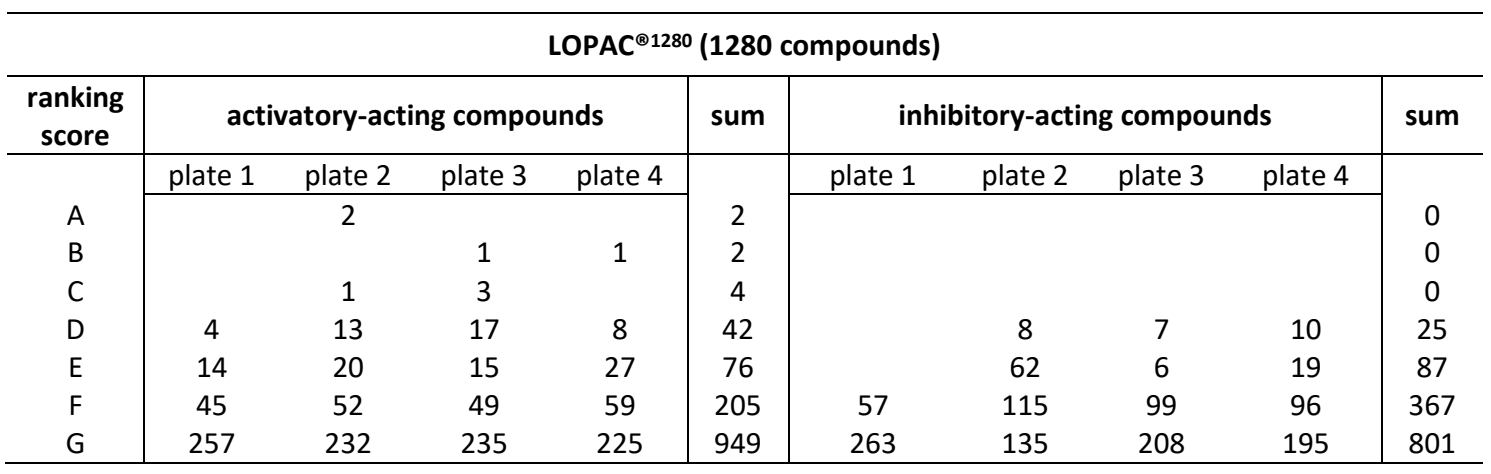

Table 3.2: The ENZO ${ }^{\circledR 774}$ library compounds sorted according to their ranking score.

\begin{tabular}{|c|c|c|c|c|c|c|c|c|}
\hline \multicolumn{9}{|c|}{ ENZO $^{\otimes 774}$ (774 compounds) } \\
\hline \multirow[t]{2}{*}{$\begin{array}{c}\text { ranking } \\
\text { score }\end{array}$} & \multicolumn{3}{|c|}{ activatory-acting compounds } & \multirow[t]{2}{*}{ sum } & \multicolumn{3}{|c|}{ inhibitory-acting compounds } & \multirow[t]{2}{*}{ sum } \\
\hline & plate 1 & plate 2 & plate 3 & & plate 1 & plate 2 & plate 3 & \\
\hline A & 1 & & & 1 & & & & 0 \\
\hline $\mathrm{B}$ & & & & 0 & & & & 0 \\
\hline C & 1 & & & 1 & & & & 0 \\
\hline $\mathrm{D}$ & 6 & 10 & 2 & 18 & 6 & 5 & 6 & 17 \\
\hline$E$ & 9 & 9 & 5 & 23 & 1 & 18 & 10 & 29 \\
\hline $\mathrm{F}$ & 51 & 31 & 27 & 109 & 141 & 80 & 79 & 300 \\
\hline G & 252 & 239 & 131 & 622 & 172 & 186 & 70 & 428 \\
\hline
\end{tabular}

\subsubsection{Hit compound conformation}

In order to verify the effect of 18 selected compounds on SEAP secretion, I manually performed a conformational screening in a 96-well format (chapter 2.39.2). Enzymatic SEAP signals of compounds from LOPAC $^{\circledR 1280}$ (chapter 8.6.2, Fig.8-13A) and ENZO ${ }^{\circledR 74}$ (Fig.8-13B) libraries I normalized to the corresponding cell number values, detected via CellTiter-Glo ${ }^{\circledR}$ assay (Fig.8-13C, D). Normalized SEAP signals I displayed as a fold change in SEAP secretion level relative to $\mathrm{MO}$ control condition, set as 1 (Fig.3-15).

SEAP secretion was significantly elevated in response to $M 1$ treatment, as seen during HTS (Fig.3-15A, B, blue bars). SEAP secretion level was also significantly upregulated relative to M1 solvent control by A-ranked hit compounds GSK 1210151 (Fig.3-15A) and vorinostat (Fig.3-15B). Also the A-ranked compound Ro 11-1464 displayed an activatory activity upregulating SEAP secretion (Fig.3-15A). The remaining activatory-acting compounds failed to show their significant effect on SEAP secretion (Fig.3-15A, B, red bars). Also inhibitory-acting compounds from LOPAC ${ }^{\circledR 1280}$ and ENZO ${ }^{\circledR 774}$ libraries failed to significantly reduce SEAP secretion relative to M1 solvent control (Fig.3-15A, B, green bars). Lasofoxifene tartrate, sanguinarine chloride, auranofin, and mitoxantrone were cell 
Results

Table 3.3: List of compounds derived from the high-throughput screening of LOPAC ${ }^{\circledR 1280}$ and ENZO ${ }^{\circledR 774}$ libraries.

\begin{tabular}{|c|c|c|c|c|c|c|}
\hline$\#$ & compound & pharmacological effect & $\begin{array}{l}\text { library } \\
\text { ID }\end{array}$ & $\begin{array}{c}\text { SD } \\
\text { (read } \\
1 \text { ) }\end{array}$ & $\begin{array}{c}\text { SD } \\
\text { (read } \\
2 \text { ) } \\
\end{array}$ & $\begin{array}{l}\text { ranking } \\
\text { score }\end{array}$ \\
\hline \multicolumn{7}{|c|}{ activatory-acting compounds } \\
\hline \multirow[t]{2}{*}{1} & GSK & bromodomain inhibitor & אמתמתו & 3 & 2 & 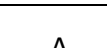 \\
\hline & $1210151 \mathrm{~A}$ & & LUPAC & 3 & 3 & A \\
\hline 2 & Ro 11-1464 & thienotriazolodiazepine & LOPAC & 3 & 3 & $A$ \\
\hline 3 & vorinostat & histone deacetylase inhibitor & ENZO & 3 & 3 & A \\
\hline \multirow[t]{2}{*}{4} & Ro 61-8048 & kynurenine 3-hydroxylase and & & & & \\
\hline & & $\begin{array}{l}\text { kynurenine 3-monooxygenase } \\
\text { (KMO) inhibitor }\end{array}$ & LOPAC & 3 & 2 & B \\
\hline 5 & $\begin{array}{l}\text { danshensu } \\
\text { sodium salt }\end{array}$ & $\begin{array}{l}\text { apoptosis inhibitor via Akt and } \\
\text { ERK1/2 phosphorylation }\end{array}$ & LOPAC & 2 & 3 & B \\
\hline 6 & leflunomide & DMARD, treatment of RA & ENZO & 3 & 1 & C \\
\hline 7 & $\begin{array}{l}\text { 13-cis-re- } \\
\text { tinoic acid }\end{array}$ & $\begin{array}{l}\text { anti-inflammatory and anti-tumor } \\
\text { agent }\end{array}$ & LOPAC & 0 & 3 & D \\
\hline 8 & bropirimine & $\begin{array}{l}\text { oral immunostimulant, interferon } \\
\text { production inducer }\end{array}$ & LOPAC & 0 & 3 & $\mathrm{D}$ \\
\hline \multirow[t]{2}{*}{9} & zileuton & $\begin{array}{l}\text { 5-lipoxygenase inhibitor, treat- } \\
\text { ment of asthma }\end{array}$ & ENZO & 3 & -1 & D \\
\hline & \multicolumn{6}{|c|}{ inhibitory-acting compounds } \\
\hline \multirow[t]{2}{*}{10} & lasofoxifene & non-steroidal, 3rd generation se- & & & & \\
\hline & tartrate & $\begin{array}{l}\text { lective estrogen receptor modula- } \\
\text { tor (SERM) }\end{array}$ & LOPAC & -2 & -1 & D \\
\hline \multirow[t]{2}{*}{11} & sanguinarine & benzophenanthridine alkaloid; & & & & \\
\hline & chloride & $\begin{array}{l}\text { anti-inflammatory and anti-oxi- } \\
\text { dant agent }\end{array}$ & LOPAC & -2 & -1 & D \\
\hline 12 & auranofin & anti-inflammatory agent & ENZO & -2 & -2 & $\mathrm{D}$ \\
\hline 13 & $\begin{array}{l}\text { mitoxant- } \\
\text { rone }\end{array}$ & Immune-suppresive action & ENZO & -2 & -2 & D \\
\hline \multirow[t]{2}{*}{14} & ketorolac & non-steroidal anti-inflammatory & & & & \\
\hline & $\begin{array}{l}\text { trometha- } \\
\text { mine }\end{array}$ & drug & ENZO & 0 & -1 & $\mathrm{E}$ \\
\hline 15 & fluocinonide & glucocorticoid & ENZO & 0 & -1 & $E$ \\
\hline 16 & amcinonide & corticosteroid & ENZO & 0 & -1 & $E$ \\
\hline 17 & roscovitine & MAPK inhibitor & LOPAC & -1 & 0 & $\mathrm{~F}$ \\
\hline 18 & halcinonide & corticosteroid & ENZO & -1 & 0 & $F$ \\
\hline
\end{tabular}

toxic at the selected treatment condition, strongly affecting cell numbers and therefore SEAP secretion (Fig.8-13C, D).

All 18 selected compounds were also characterized by qPCR: gene expression of endogenous CEBPD and CEBPD::SEAP reporter were quantified (data not shown). mRNA expression of CEBPD::SEAP failed to correlate to the SEAP readout for $\mathrm{B}_{-}, \mathrm{C}_{-}, \mathrm{D}-$, and F-ranked activatory-acting and inhibitory-acting compounds with SEAP signals within three SDs from the solvent control. Correlation between CEBPD::SEAP gene expression 


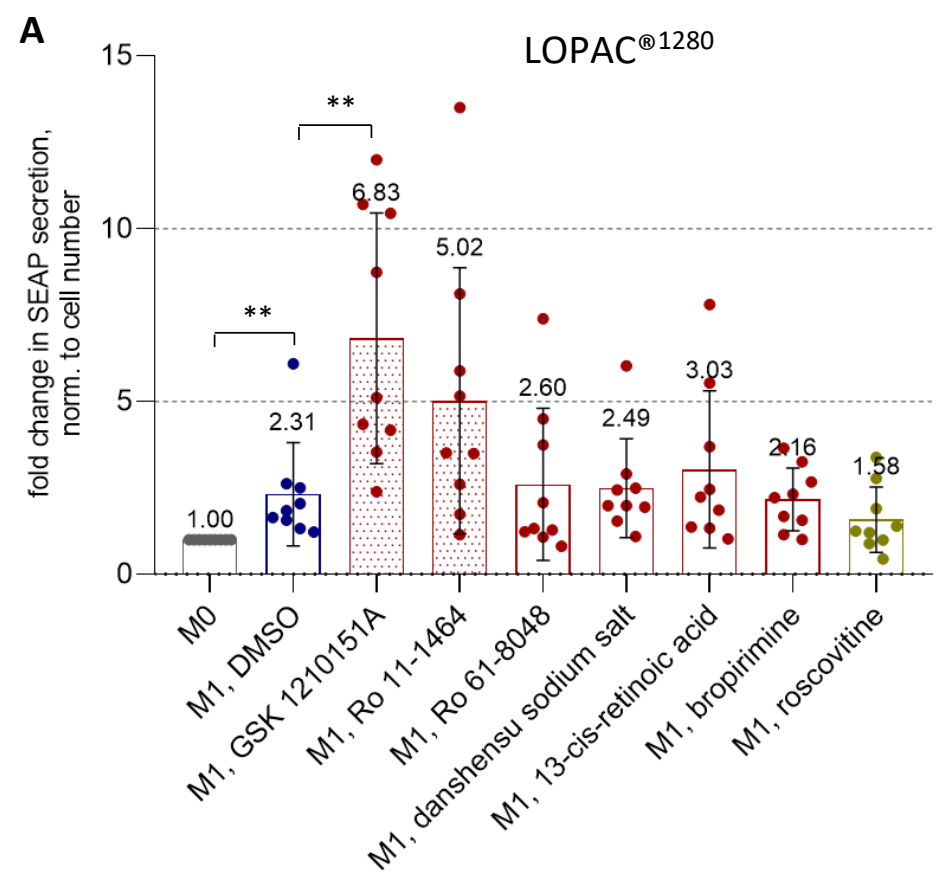

B

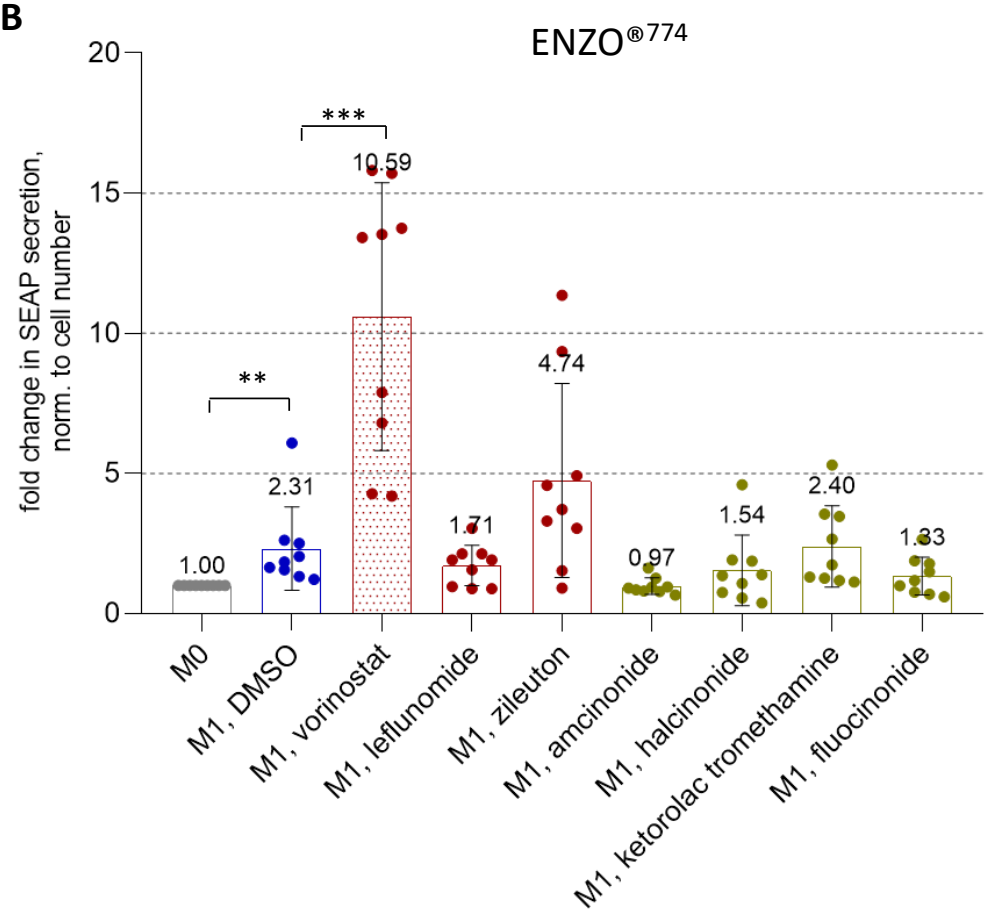

Figure 3-15: Conformational analysis of selected compounds from LOPAC ${ }^{\circledR 1280}$ and ENZO ${ }^{\circledR 774}$ libraries. A, B: Selected compounds from $\operatorname{LOPAC}^{\circledR 1280}$ (A) and ENZO ${ }^{\circledR 774}$ (B) libraries were screened in 96-well format as described previously (chapter 2.39.2). SEAP signals of activatory- (red bars) and inhibitory-acting (green bars) compounds (Fig.8-11A, B) were normalized to corresponding cell number values (Fig.8-11C, D) and are shown here as fold changes in SEAP secretion level relative to $\mathrm{MO}$ control, set as 1 (grey bars). Difference in SEAP secretion levels between M0 control and M1 solvent control was analyzed via Wilcoxon signed-rank test (1.00 as hypothetical value). Differences in SEAP secretion levels between M1 solvent control and M1 compound treatment conditions were analyzed via non-parametric Mann-Whitney test. Data represent mean \pm SD of nine single wells per condition. ${ }^{* *} p<0.005 ; * * * p<0.001$. 
and SEAP readout was observed for all three A-ranked compounds with signals above three SDs: GSK 1210151A, Ro 11-1464, and vorinostat, characterizing the generated screening assay as sensitive and specific for CEBPD-activatory compounds.

GSK $1210151 \mathrm{~A}$ and Ro 11-1464 are bromo-domain and extra-terminal motif (BET) inhibitors, whereas vorinostat is a histone deacetylase (HDAC) inhibitor. The control compound TSA, which reproducibly upregulated SEAP secretion during HTS, is also an HDAC inhibitor with a similar structure to vorinostat. Confirmed hit compounds and TSA were further characterized according to their pharmacological activity in target cells.

\subsection{Epigenetically active hit compounds modulate CEBPD gene expression}

GSK 1210151A, Ro 11-1464, vorinostat, and TSA I characterized according to their ability to modulate gene expression in the chosen cellular context of inflammation. To do so, I observed hit compound-mediated changes in mRNA expression of following genes in M1-polarized THP-1 reporter M $\varphi$ : endogenous CEBPD, reporter CEBPD::SEAP, interleukin 6 (IL-6), interleukin 1 beta (IL-1ß), and CC-chemokine ligand 2 (CCL2) (chapter 2.39.4). As the defined CEBPD promoter contains a restricted number of known and proposed regulatory sequences, the hit compound effect on expression of reporter CEBPD::SEAP may differ from that of endogenous CEBPD. Therefore, it was important to monitor the expression of both CEBPD and CEBPD::SEAP. Should the expression of these genes differ in response to the hit compound treatment, the relevant regulatory sequences can be identified as the sequence of the defined CEBPD promoter is known. Also the expression of IL- 6 and IL-1ß selected genes that belong to the downstream agents of the C/EBPS signaling was monitored in target cells. Thus, IL-6, which elevated cytokine production is one of the hallmarks of RA, ${ }^{164}$ is a direct target gene of C/EBP $\delta$ in LPS-treated murine primary $M \varphi .{ }^{71}$ The $\mathrm{C} / \mathrm{EBP} \delta$ binding motifs were also identified in the functional regulatory regions of IL-1ß expressed by myelomonocytic lineage cells. ${ }^{118}$ Finally, the selected $\mathrm{CCL} 2$ gene serves as M1 polarization marker in THP-1 reporter $\mathrm{M} \varphi .^{219}$

The expression of IL-6, IL-1ß, and CCL2 pro-inflammatory genes was significantly activated and constantly upregulated in response to the $\mathrm{M} 1$ treatment relative to $\mathrm{MO}$ control (Fig.3-16A, Fig.3-17A, Fig.3-18A, IL-6, IL-1ß, CCL2, 4h, 24h, blue bars). The sustained 
upregulation of these pro-inflammatory genes indicated the expected M1-polarization of THP-1 reporter M $\varphi$. Gene expression of endogenous CEBPD was significantly up to 6-fold activated (Tables 3.4, 3.5, 3.6) relative to M0 control, 4 hours after M1 treatment (Fig.3-16A, Fig.3-17A, Fig.3-18A, CEBPD, 4h, blue bars). At the later time point, the upregulation of endogenous CEBPD decayed displaying low 1.3-fold significant (Fig.3-16A, Fig.3-18A, CEBPD, 24h, blue bars) or not significant (Fig.3-17A, CEBPD, 24h, blue bar) difference to M0 control (Tables $3.4,3.5,3.6)$. The M1 treatment-activated mRNA expression of CEBPD::SEAP reporter mirrored that of endogenous CEBPD and displayed up to 5 -fold activation relative to $\mathrm{MO}$ control (Tables 3.4, 3.5, 3.6), at the early time point (Fig.3-16A, Fig.3-17A, Fig.3-18A, CEBPD::SEAP, 4h, blue bars). In contrast to endogenous CEBPD, gene expression of CEBPD::SEAP reporter was significantly up to 3-fold upregulated by M1 treatment (Tables 3.4, 3.5, 3.6), at the late time point (Fig.3-16A, Fig.3-17A, Fig.3-18A, CEBPD::SEAP, 24h, blue bars). Elevated SEAP enzymatic activity in cellular supernatant indicated the upregulated CEBPD::SEAP gene expression in response to M1 treatment, 24 h post-treatment (Fig.3-16B, Fig.3-17B, Fig.3-18B, blue bars). The enzymatic SEAP activity represented a bulk reporter signal accumulated over time upon CEBPD target promoter activation.

\section{GSK $1210151 A$}

Endogenous CEBPD and CEBPD::SEAP reporter genes displayed a similar significant upregulation (Table 3.4) in their expression by GSK 1210151A, relative to M1 DMSO control at the early time point (Fig.3-16A, CEBPD::SEAP, CEBPD, 4h, red bars). However, at the later time point GSK 1210151A effected CEBPD and CEBPD::SEAP gene expression differently. While GSK 1210151A-upregulated mRNA expression of CEBPD::SEAP further increased and was over 15-times higher relative to M1 DMSO control, endogenous CEBPD mRNA expression did not correlate, being 1.8-times higher (Table 3.4), 24 hours after M1 treatment (Fig.3-16A, CEBPD::SEAP, CEBPD, 24h, red bars). Remarkably, at the same time the expression of IL-1ß, IL-6, and CCL2 was significantly decreased by GSK 1210151A relative to M1 DMSO control (Fig.3-16A, IL-6, IL-1ß, CCL2, 24h, red bars). Gene expression of IL- 6 and CCL2 was also significantly reduced by GSK $1210151 \mathrm{~A}$ at the early time point (Fig.3-16A, IL-6, CCL2, 4h, red bars), displaying its anti-inflammatory action. In contrary, 4 hours after M1 treatment, GSK 1210151A significantly unregulated 
Results

A

$4 \mathrm{~h}$
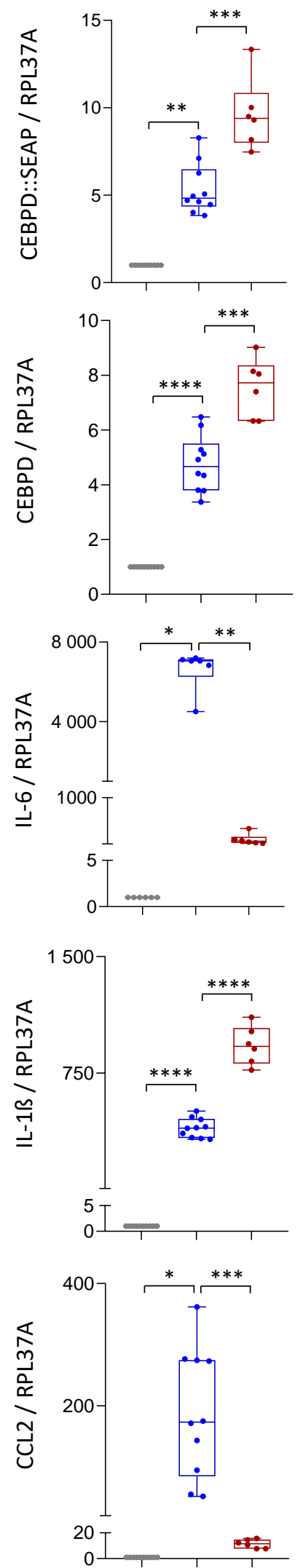

$24 \mathrm{~h}$
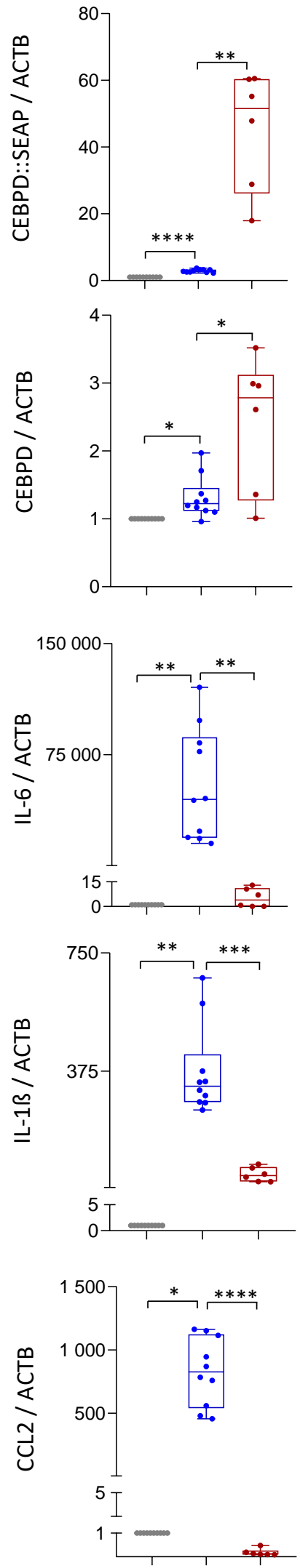

B SEAP assay, 24h

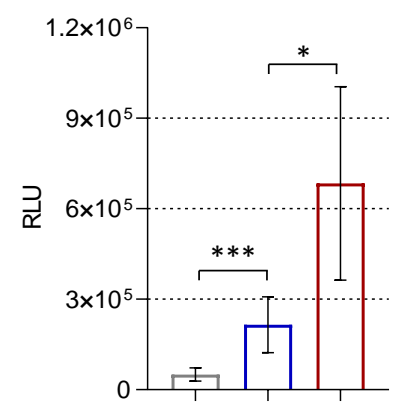

legend:

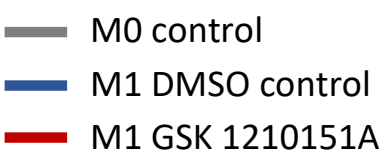

Figure 3-16: Effect of BET inhibitor GSK 1210151A on mRNA expression and SEAP secretion. THP-1 reporter cells were PMA-differentiated, seeded in a 24-well format, pre-treated with either DMSO (solvent) or $10 \mu \mathrm{M}$ GSK $1210151 \mathrm{~A}$ for $1 \mathrm{~h}$, and M1-polarized with $0.1 \mu \mathrm{g} / \mathrm{mL} \mathrm{LPS}+20 \mathrm{ng} / \mathrm{mL}$ IFN-g (M1 treatment) for $4 \mathrm{~h}$ or $24 \mathrm{~h}$. Changes in gene expression (A) and SEAP secretion (B) were analyzed in lysates and cellular 
supernatants of non-polarized (M0 control), M1 polarized DMSO (M1 DMSO control) and GSK 1210151A pre-treated (M1 GSK 1210151A) THP-1 reporter $\mathrm{M} \varphi$, at indicated time points. A: Gene expression analysis was performed via RT-qPCR ( $\triangle \Delta C_{t}$ method) using RPL37A and ACTB house-keeping genes for $4 \mathrm{~h}$ and $24 \mathrm{~h}$ time points, respectively (chapter 8.6.4, Fig.8-15). Fold change in gene expression is displayed relative to $\mathrm{MO}$ control, set as 1 . Differences between $\mathrm{M} 0$ and $\mathrm{M} 1 \mathrm{DMSO}$ controls were analyzed via Wilcoxon signed-rank test (1.00 as hypothetical value). GSK $1210151 \mathrm{~A}$-mediated changes in gene expression were analyzed relative to M1 DMSO control via unpaired t test with (for normally distributed data and different SDs) and without (for normally distributed data and equal SDs) Welch's correction or Mann-Whitney test (compared ranks; for not normally distributed data). The median and range of three independent experiments with 2-3 wells per condition are presented in Box-Whisker plots. B: Changes in SEAP secretion were analyzed relative to M1 DMSO control via Brown-Forsythe and Welch ANOVA test with Dunnett's correction for multiple comparisons. Data represent mean \pm SD of three independent experiments with $2-3$ wells per condition. ${ }^{*} p<0.05 ;{ }^{*} p<0.005 ; * * * p<0.001 ; * * * p<0.0001$.

Table 3.4: qPCR quatified mRNA expression levels of selected genes in M1-polarized and DMSO or GSK 1210151A pre-treated THP-1 M $\varphi$.

\begin{tabular}{llcc}
\hline gene & condition & 4h, mean \pm SD & 24h, mean \pm SD \\
\hline \multirow{2}{*}{ CEBPD::SEAP } & M1 DMSO & $5.3 \pm 1.4$ & $2.9 \pm 0.5$ \\
& M1 GSK 1210151A & $9.6 \pm 2.0$ & $45.1 \pm 17.8$ \\
\hline \multirow{2}{*}{ CEBPD } & M1 DMSO & $4.8 \pm 1.0$ & $1.3 \pm 0.3$ \\
& M1 GSK 1210151A & $7.5 \pm 1.1$ & $2.4 \pm 1.0$ \\
\hline \multirow{2}{*}{ IL-6 } & M1 DMSO & $6,625.0 \pm 1,045.0$ & $54,346.0 \pm 37,792.0$ \\
\hline \multirow{2}{*}{ IL-1ß } & M1 GSK 1210151A & $102.3 \pm 118.1$ & $5.3 \pm 5.7$ \\
\hline \multirow{2}{*}{ CCL2 } & M1 DMSO & $396.0 \pm 62.4$ & $373.00 \pm 141.8$ \\
& M1 GSK 1210151A & $927.1 \pm 124.2$ & $46.6 \pm 22.7$ \\
\hline
\end{tabular}

gene expression of IL-1ß relative to M1 DMSO control (Fig.3-16A, IL-1ß, 4h, red bar). Increase in SEAP enzymatic activity in cellular supernatants also indicated the activatory effect of GSK 1210151A on CEBPD::SEAP gene expression (Fig.3-16B, red bar).

Ro 11-1464

Ro 11-1464 elicited a significant increase in expression of endogenous CEBPD and CEBPD::SEAP reporter genes relative to M1 DMSO control, 4 hours after M1 treatment (Fig.3-17A, CEBPD::SEAP, CEBPD, 4h, red bars). Gene expression of endogenous CEBPD differed from that of reporter CEBPD::SEAP gene in response to Ro 11-1464 treatment at the later time point. Whereas mRNA expression of reporter CEBPD::SEAP displayed an approximately 2-fold upregulation (Table 3.5) by Ro 11-1464, endogenous CEBPD mRNA expression was not different to M1 DMSO control, 24 hours after M1 treatment (Fig.3-17A, CEBPD::SEAP, CEBPD, 24h, red bars). At the same time point, IL-6, IL-1ß, and 
A
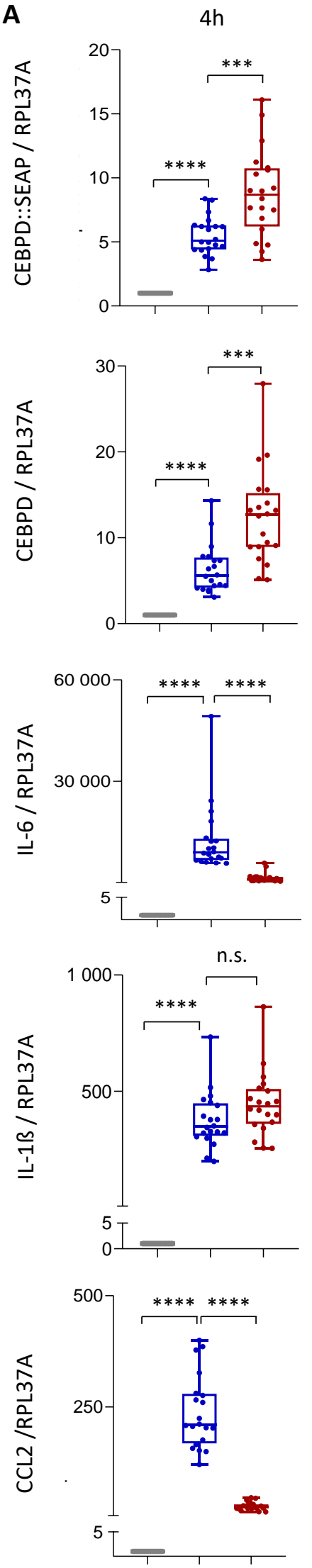

$24 \mathrm{~h}$
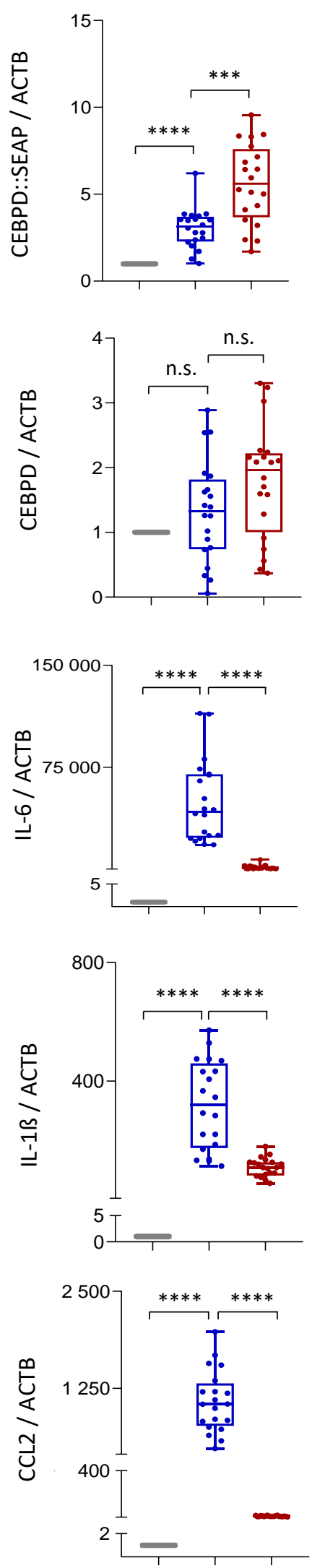

B SEAP assay, $24 \mathrm{~h}$

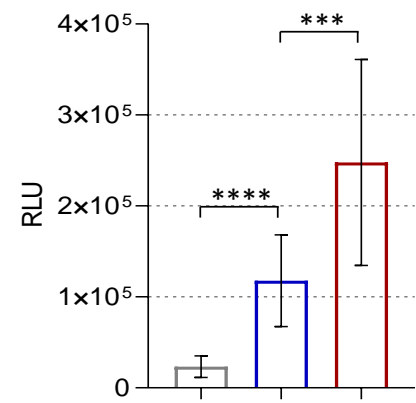

legend:

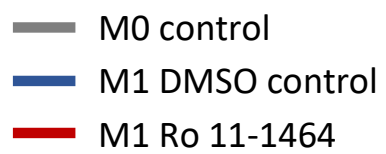

Figure 3-17: Effect of BET inhibitor Ro 11-1464 on mRNA expression and SEAP secretion. THP-1 reporter cells were PMA-differentiated, seeded in a 24-well format, pre-treated with either DMSO (solvent) or 10 $\mu \mathrm{M}$ Ro $11-1464$ for $1 \mathrm{~h}$, and M1-polarized with $0.1 \mu \mathrm{g} / \mathrm{mL}$ LPS $+20 \mathrm{ng} / \mathrm{mL}$ IFN-g (M1 treatment) for $4 \mathrm{~h}$ or 24h. Changes in gene expression (A) and SEAP secretion (B) were analyzed in lysates and cellular super- 
natants of non-polarized (M0 control), M1-polarized DMSO (M1 DMSO control) and Ro 11-1464 pretreated (M1 Ro 11-1464) THP-1 reporter $\mathrm{M} \varphi$, at indicated time points. A: Gene expression analysis was performed via RT-qPCR ( $\Delta \Delta \mathrm{C}_{\mathrm{t}}$ method) using RPL37A and ACTB house-keeping genes for $4 \mathrm{~h}$ and $24 \mathrm{~h}$ time points, respectively (chapter 8.6.4, Fig.8-15). Fold change in gene expression is displayed relative to $\mathrm{MO}$ control, set as 1 . Differences between M0 and M1 DMSO controls were analyzed via Wilcoxon signedrank test (1.00 as hypothetical value). Ro 11-1464-mediated changes in gene expression were analyzed relative to M1 DMSO control via unpaired t test with (for normally distributed data and different SDs) and without (for normally distributed data and equal SDs) Welch's correction or Mann-Whitney test (compared ranks; for not normally distributed data). The median and range of five independent experiments with 4 wells per condition are presented in Box-Whisker plots. B: Changes in SEAP secretion were analyzed relative to M1 DMSO control via Kruskal-Wallis test with Dunn's correction for multiple comparisons. Data represent mean $\pm S D$ of five independent experiments with 4 wells per condition. n.s.: not significant; $* p<0.05 ; * * * p<0.001 ; * * * * p<0.0001$.

Table 3.5: qPCR quatified mRNA expression levels of selected genes in M1-polarized and DMSO or Ro 11-1464 pre-treated THP-1 M $\varphi$.

\begin{tabular}{llcc}
\hline gene & condition & 4h, mean \pm SD & 24h, mean \pm SD \\
\hline \multirow{2}{*}{ CEBPD::SEAP } & M1 DMSO & $5.5 \pm 1.5$ & $3.0 \pm 1.1$ \\
& M1 Ro 11-1464 & $8.8 \pm 3.4$ & $5.6 \pm 2.3$ \\
\hline \multirow{2}{*}{ CEBPD } & M1 DMSO & $6.3 \pm 2.8$ & $1.3 \pm 0.8$ \\
& M1 Ro 11-1464 & $12.4 \pm 5.5$ & $1.8 \pm 0.9$ \\
\hline \multirow{2}{*}{ IL-6 } & M1 DMSO & $12,432.0 \pm 10092.0$ & $49,344.0 \pm 30143.0$ \\
& M1 Ro 11-1464 & $1,364.0 \pm 1426.0$ & $1,317.0 \pm 1571.0$ \\
\hline \multirow{2}{*}{ IL-1ß } & M1 DMSO & $344.8 \pm 119.0$ & $319.7 \pm 148.8$ \\
& M1 Ro 11-1464 & $445.3 \pm 138.6$ & $108.8 \pm 31.4$ \\
\hline \multirow{2}{*}{ CCL2 } & M1 DMSO & $237.2 \pm 82.8$ & $1,079.0 \pm 395.6$ \\
& M1 Ro 11-1464 & $26.6 \pm 9.0$ & $10.9 \pm 3.9$ \\
\hline
\end{tabular}

CCL2 gene expression was significantly reduced by Ro 11-1464 (Fig.3-17A, IL-6, IL-1ß, CCL2, 24h, red bars). mRNA expression of IL-6 and CCL2 was also significantly suppressed by Ro 11-1464 4 hours after M1 treatment (Fig.3-17A, IL-6, CCL2, 4h, red bars), displaying its anti-inflammatory action. At the early time point, Ro 11-1464 showed no significant effect on IL-1ß gene expression (Fig.3-17A, IL-1ß, 4h, red bar). Significant upregulation in SEAP enzymatic activity in cellular supernatants also indicated the activatory effect of Ro 11-1464 on CEBPD::SEAP gene expression (Fig.3-17B, red bar).

\section{Trichostatin A and vorinostat}

Vorinostat and TSA displayed similar effects on expression of selected genes relative to M1 DMSO control at the early time point (Fig.3-18A, 4h, red and orange bars). Remarkably, the M1 treatment-activated gene expression of endogenous CEBPD was completely abolished, while this of CEBPD::SEAP reporter was significantly 1.5 -fold upregu- 
lated (Table 3.6) by TSA and vorinostat at the 4 hour time point (Fig.3-18A, CEBPD::SEAP, CEBPD, $4 \mathrm{~h}$, red and orange bars). At the 24 hour time point, both compounds displayed no significant effect on gene expression of endogenous CEBPD (Fig.3-18A, CEBPD, 24h, red and orange bars). At the same time point, mRNA expression of reporter CEBPD::SEAP was upregulated 50 -fold (Table 3.6) by vorinostat relative to the M1 DMSO control (Fig.3-18A, CEBPD::SEAP, 24h, red bar). However, TSA caused no significant effect on CEBPD::SEAP gene expression, at the 24 hour time point (Fig.3-18A, CEBPD::SEAP, 24h, orange bar). mRNA expression of CCL2 was significantly suppressed by vorinostat and TSA at both time points tested (Fig.3-18A, CCL2, 4h, 24h, red and orange bars). The effects of vorinostat and TSA on gene expression of endogenous CEBPD, CEBPD::SEAP reporter, and CCL2 were further analysed during a longitudinal study (chapter 8.7, Fig.8-16A, B, C). Gene expression of IL-6, being significantly downregulated by TSA and vorinostat at both time points tested (Fig.3-18A, IL-6, 4h, 24h, red and orange bars), indicated their anti-inflammatory action in target cells. TSA and vorinostat upregulated $\mathrm{IL}-1 ß$ at $4 \mathrm{~h}$ and displayed no effect at $24 \mathrm{~h}$ time points (Fig.3-18A, IL-1ß, 4h, 24h, red and orange bars). Elevated SEAP enzymatic activity in cellular supernatants also indicated the activatory effect of TSA and vorinostat on CEBPD::SEAP gene expression (Fig.3-18B, red and orange bars; chapter 8.7, Fig.8-16D). 
A

A $4 h$
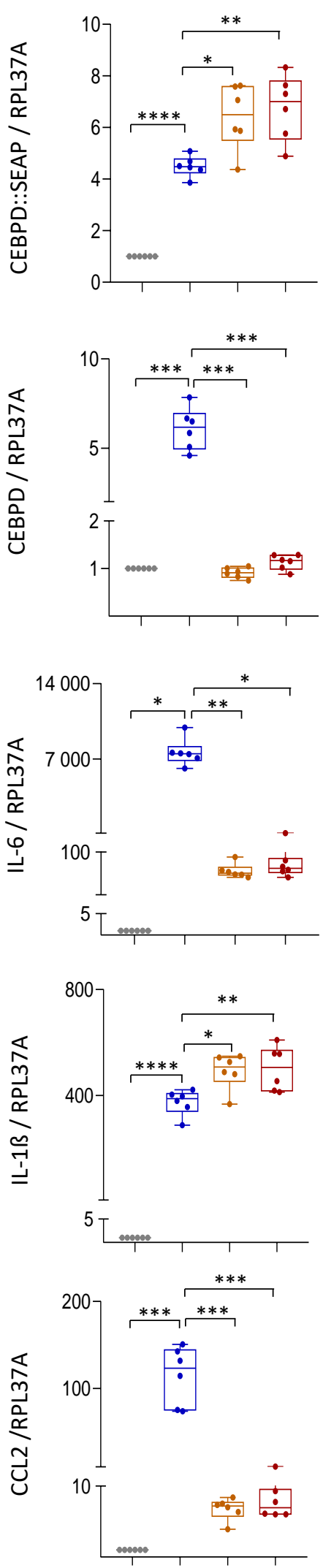

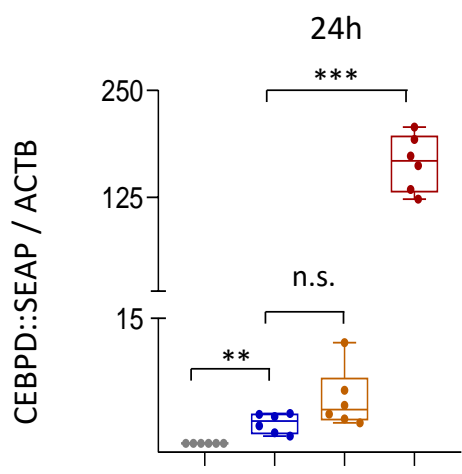

B

SEAP assay, $24 \mathrm{~h}$
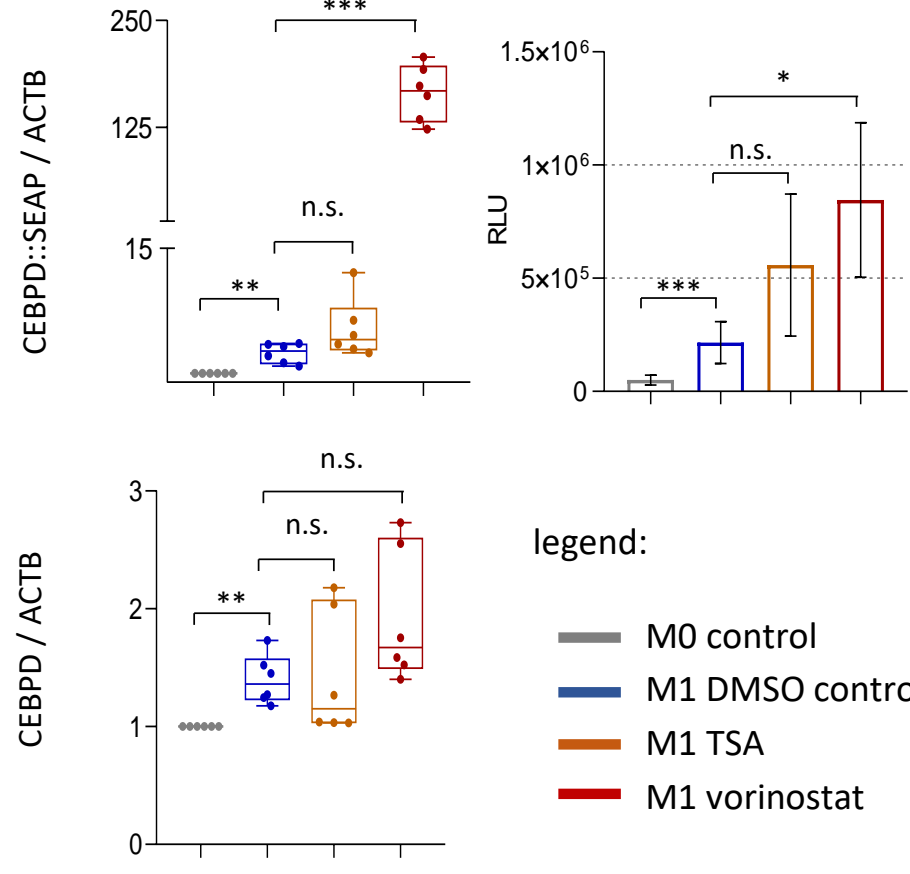

legend:

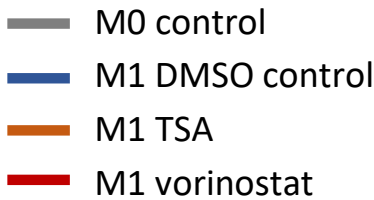

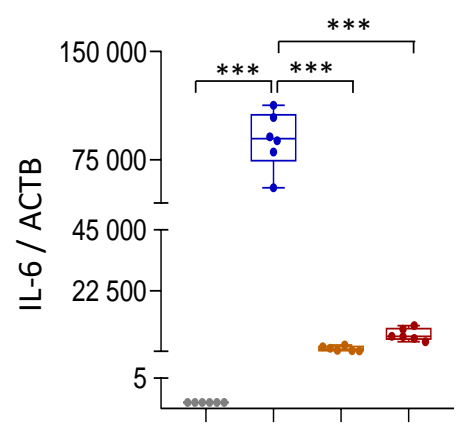
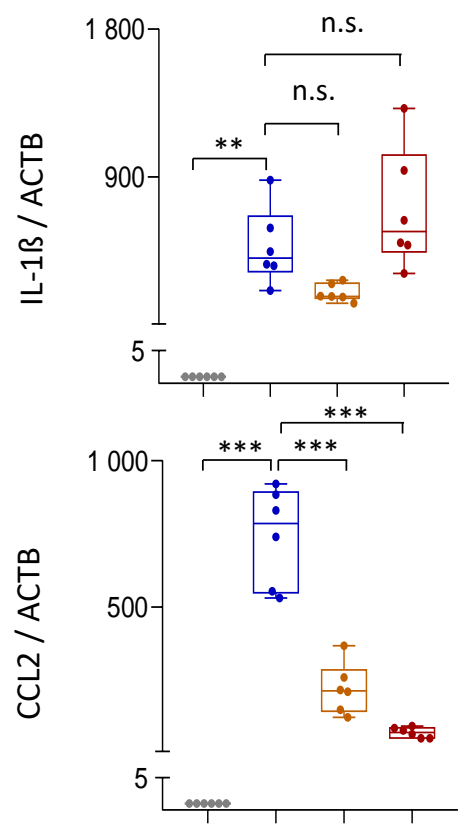

Figure 3-18: Effect of HDAC inhibitors TSA and vorinostat on mRNA expression and SEAP secretion. THP-1 reporter cells were PMA-differentiated, seeded in a 24-well format, pre-treated with either DMSO (solvent), $10 \mu \mathrm{M}$ vorinostat, or $0.5 \mu \mathrm{M}$ TSA for $1 \mathrm{~h}$, and M1-polarized with $0.1 \mu \mathrm{g} / \mathrm{mL} \mathrm{LPS}+20 \mathrm{ng} / \mathrm{mL}$ IFN-g (M1 treatment) for $4 \mathrm{~h}$ or $24 \mathrm{~h}$. Changes in gene expression (A) and SEAP secretion (B) were analyzed 
in lysates and cell culture supernatants of non-polarized (M0 control), M1-polarized DMSO (M1 DMSO control), vorinostat (M1 vorinostat), and TSA pre-treated (M1 TSA) THP-1 reporter $M \varphi$, at indicated time points. A: Gene expression analysis was performed via RT-qPCR ( $\Delta \Delta \mathrm{C}_{t}$ method) using RPL37A and ACTB house-keeping genes for $4 \mathrm{~h}$ and $24 \mathrm{~h}$ time points, respectively (chapter 8.6.4, Fig.8-15). Fold change in gene expression is displayed relative to M0 control, set as 1 . Differences between M0 and M1 DMSO controls were analyzed via Wilcoxon signed-rank test (1.00 as hypothetical value). Compound-mediated changes in gene expression were analyzed relative to M1 DMSO control via ordinary one-way ANOVA (for normally distributed data and equal SDs), Brown-Forsythe and Welch ANOVA test with Dunnett's correction (for normally distributed data and different SDs), or Kruskal-Wallis test with Dunn's correction for multiple comparisons (for not normally distributed data). The median and range of three independent experiments with 2 wells per condition are presented in Box-Whisker plots. B: Changes in SEAP secretion were analyzed relative to M1 DMSO control via Brown-Forsythe and Welch ANOVA test with Dunnett's correction for multiple comparisons. Data represent mean \pm SD of three independent experiments with 2 wells per condition. n.s.: not significant; ${ }^{*} p<0.05 ; * * p<0.005 ; * * *<0.001 ; * * * p<0.0001$.

Table 3.6: qPCR quatified mRNA expression levels of selected genes in M1-polarized and DMSO, TSA, or vorinostat pre-treated THP-1 M $\varphi$.

\begin{tabular}{|c|c|c|c|}
\hline gene & condition & $4 \mathrm{~h}$, mean $\pm S D$ & $24 \mathrm{~h}$, mean \pm SD \\
\hline \multirow{3}{*}{ CEBPD::SEAP } & M1 DMSO & $4.5 \pm 0.4$ & $3.3 \pm 1.1$ \\
\hline & M1 TSA & $6.4 \pm 1.3$ & $6.0 \pm 3.4$ \\
\hline & M1 vorinostat & $6.8 \pm 1.3$ & $165.4 \pm 32.7$ \\
\hline \multirow{3}{*}{ CEBPD } & M1 DMSO & $6.1 \pm 1.2$ & $1.4 \pm 0.2$ \\
\hline & M1 TSA & $0.9 \pm 0.1$ & $1.4 \pm 0.5$ \\
\hline & M1 vorinostat & $1.1 \pm 0.2$ & $1.9 \pm 0.6$ \\
\hline \multirow{3}{*}{ IL-6 } & M1 DMSO & $7,617.0 \pm 1,258.0$ & $88,8654.0 \pm 19,906.0$ \\
\hline & M1 TSA & $58.1 \pm 16.1$ & $1,014.0 \pm 926.7$ \\
\hline & M1 vorinostat & $68.7 \pm 20.1$ & $6,189.0 \pm 2,234.0$ \\
\hline \multirow{3}{*}{ IL-1ß } & M1 DMSO & $374.3 \pm 47.9$ & $234.2 \pm 53.8$ \\
\hline & M1 TSA & $492.5 \pm 67.1$ & $475.5 \pm 195.0$ \\
\hline & M1 vorinostat & $501.9 \pm 83.6$ & $698.8 \pm 368.4$ \\
\hline \multirow{3}{*}{ CCL2 } & M1 DMSO & $114.9 \pm 33.4$ & $743.2 \pm 167.5$ \\
\hline & M1 TSA & $6.8 \pm 1.5$ & $220.4 \pm 87.1$ \\
\hline & M1 vorinostat & $7.6 \pm 1.9$ & $69.8 \pm 17.8$ \\
\hline
\end{tabular}




\section{Discussion}

\subsection{Translational potential of the developed screening assay}

\subsubsection{Phenotypic screening assay: evaluation of the chosen cellular system}

Disease relevance of cell-based assays depends on expression of characteristic proteins to recapitulate proper biologic cell organization ${ }^{4}$. As a part of an interactive cellular network, M1-polarized $\mathrm{M} \varphi$ are central in inflammatory RA condition due to their production of disease-driving cytokines and chemokines (cf. chapter 1.3.3). Facing complexity of cellular interaction in RA, M1-polarized THP-1 M $\varphi$ are able to represent only a restricted inflammatory aspect of RA pathology.

Generally, cell lines display poor correlation to patient-derived cells due to the differences in the genetic background, ${ }^{4}$ lack of endogenous stimuli complexes and cellular cointeractions. ${ }^{197}$ The THP-1 cell line has overall diploid character displaying triploidy for chromosomes 1, 6, 8, and 9, monoploidy for chromosome 10, as well as complete deletion of chromosome $X$, accompanied by substantial chromosomal rearrangements. ${ }^{220}$ THP-1 cells require PMA, a non-physiological protein kinase C (PKC) activator, ${ }^{216,221}$ to induce their differentiation to $\mathrm{M} \varphi$. PMA-induced cell differentiation displays its individual characteristics regarding differentiation degree, cellular morphology, and cell surface receptor expression. ${ }^{221-223}$ In this study, PMA- differentiation of THP-1 cells was performed according to the protocol, previously established in our lab. ${ }^{214}$ Based on the analysis of the established polarization markers, THP-1-derived $\mathrm{M} \varphi$ show exclusive polarization profiles in comparison to these of PBMC-derived M $\varphi .{ }^{214}$ Nevertheless, M1-polarized THP-1 M $\varphi$ can be used as a cellular model of inflammation, with regard to expression of polarization-linked genes and cell surface proteins. ${ }^{219}$

Although primary cells are suggested to improve screening assay reliability, ${ }^{4}$ THP-1 cell line displays multiple advantages in concerning cell accessibility, cell-cell contamination, and reproducibility. Thus, monocytes that originate from buffy coats of healthy adult probands can vary from 2 to $10 \%$ of PBMCs, ${ }^{224}$ disadvantageously affecting routine cell accessibility during HTS. During isolation of PBMC-derived monocytes, contamination with other blood cells like platelets needs to be considered. ${ }^{225,226}$ In contrary to primary cells displaying high variation from individual donors, THP-1 show homogeneous genetic 
background that reduces phenotypic variance and therefore facilitates reproducibility. ${ }^{225,226}$

\subsubsection{Phenotypic screening assay: evaluation of the chosen stimulus}

Intrinsic stimuli observed in patient-derived tissue display the highest disease relevance during phenotypic screening. ${ }^{4}$ Cytokine IFN-g displays high disease relevance due to its intrinsic function in RA joint ${ }^{193}$ and LPS elicits TLR4 signaling pathways discussed to contribute to synovitis during RA ${ }^{188,189}$ ( $c f$. chapter 1.3 .3 ). The chosen stimuli INF-g and LPS also induce THP-1 M $\varphi$ polarization toward a pro-inflammatory M1 state, a prominent cell type in RA joint (cf. chapter 1.3.3).

\subsubsection{Phenotypic screening assay: evaluation of the chosen readout}

Disease relevance of a readout is determined by its proximity to the functional manifestation of the chosen pathology. ${ }^{4}$ SEAP-based monitoring of CEBPD gene expression may display limited translational ability due to the relative distal proximity of CEBPD to the clinical end point. Gene expression readouts are assumed to display low translational capacity, as mechanisms that influence expression of a selected gene represent only a fraction of the mechanistic network that underlines the final cellular phenotype. ${ }^{4}$ CEBPD is one of multiple interlayers, the summary action of which underscore inflammatory RA conditions.

Nonetheless, suitability of CEBPD as target gene for screening assay readout relies on its structural and regulatory characteristics as well as on its functional implementation in inflammatory responses and RA pathology.

Direct correlation between target gene expression and functional activity of corresponding protein is the most important requirement for the chosen screening procedure. For CEBPD gene, this direct correlation is displayed to a high extent: i) CEBPD gene is intronless, producing a single C/EBPS mRNA; ii) a single full-length functional C/EBP $\delta$ protein isoform is known; iii) C/EBP $\delta$ mRNA and protein display short half-lives; iv) upon synthesis C/EBP $\delta$ protein rapidly translocates to the nucleus; v) gene expression activation represents the main mechanism for C/EBP $\delta$ regulation (cf. chapter 1.3.1). 
During inflammation, environment-responsive CEBPD influences cellular morphology and function and therefore co-defines a cell-specific phenotype. In inflammatory $М \varphi:$ i) CEBPD displays low basal gene expression levels, which are rapidly but transiently activated by IFN-g and LPS; ii) C/EBP $\delta$ controls transcription of LPS-responsive genes, amplification of NF-kB regulatory circuits, and differentiation between transient and persistent inflammatory responses; iii) activation of C/EBPS TF may also result in an anti-inflammatory $\mathrm{M} \varphi$ phenotype (cf. chapter 1.3.2.1).

CEBPD gene also shows unique functional aspects demonstrating direct links to biological disease manifestation of RA. Elevated CEBPD gene expression in RA M $\varphi$ : i) contributes to secretion of pro-inflammatory cytokines and chemokines ${ }^{132}$; ii) contributes to the attraction and infiltration of inflammatory cells, proliferation and migration of fibroblasts, and angiogenesis ${ }^{132}$; iii) directly targets IL-6 gene, one of the hallmarks of RA. ${ }^{71}$ Pharmacological modulation of CEBPD beneficially affects RA pathology ${ }^{132}$ (cf. chapter 1.3.3).

\subsubsection{Evaluation of the screening tool: multi-gene-reporter cassette $\mathbf{1 . 0}$}

Gene reporter-based assays belong to a specific category of phenotypic screening ${ }^{3}$ requiring a use of reliable and robust gene reporters. To identify CEBPD-modulating compounds, I generated the multi-gene-reporter cassette 1.0 encoding SEAP and GLuc secreted enzymatic as well as eGFP fluorescent reporter proteins (Fig.3-6A).

SEAP chemiluminescent assay displayed high assay sensitivity ( $Z^{\prime}$-factor of 0.7 for the 1,000 -fold diluted sample), high assay linearity $\left(R^{2}=0.9945\right.$ for over four 10 -fold dilutions), high assay repeat- (intra-assay CV $<10 \%$ ) and reproducibility (inter-assay CV < $5 \%$ ), detected for strong enzymatic signals (chapter 3.2.1.1, Fig.3-4). Chemiluminescent SEAP assay also displayed high assay sensitivity ( $Z^{\prime}$-factor of 0.86 for $1.25 \mu \mathrm{L}$ ) for weak enzymatic signals and low sample volume (chapter 8.4.2, Fig.8-8). In this study, SEAP served as a gene reporter to monitor CEBPD gene expression, which displays a rather weak promoter activity (chapter 8.3, Fig.8-6) resulting in relatively low SEAP reporter signals. The multi-gene-reporter cassette 1.0 was expressed under control of a defined CEBPD promoter that contains only the most proximal known regulatory sequences 
(chapter 1.3.1.3 Fig.1-3A, B). Nonetheless, reporter CEBPD::SEAP and endogenous CEBPD mRNA expression mirrored each other in response to M1 treatment (chapter 3.2.3.1, Fig.3-10).

Chemiluminescent Gluc assay displayed moderate assay sensitivity ( $Z^{\prime}$-factor of 0.8 for the 100 -fold diluted sample), moderate assay linearity $\left(R^{2}=0.9986\right.$ for over three 10 -fold dilutions), moderate assay repeatability (intra-assay CV $<15 \%$ ) and reproducibility (inter-assay CV < $15 \%$ ), detected for strong enzymatic signals (chapter 3.2.1.1, Fig.3-5). GLuc utilizes its specific substrate enabling simultaneous detection of SEAP and GLuc in the same cellular supernatant. In this study, GLuc may have served as gene reporter for normalization. During screening development, however, CMV::GLuc mRNA expression was detrimentally affected by treatment with control compounds (data not shown). The unknown adjacent regulatory sequences may influence GLuc gene expression, as the viral integration of multi-gene-reporter cassette 1.0 in the genome of THP-1 cells occurs in an uncontrolled fashion. Instead of GLuc, I used CellTiter-Glo ${ }^{\circledR}$ assay to normalize SEAP signals to the cell numbers.

In THP-1 reporter $M \varphi$, SEAP reliably correlated screening compound-mediated changes in CEBPD promoter activity to the corresponding enzymatic signals in cellular supernatants. Indicating repeatability of the generated screening assay, SEAP signals of selected 18 compounds correlated significantly from measurements in 384- (HTS) 96- (hit conformation), or 24-well (hit characterization) formats (chapter 8.6.3, Fig.8-14). Performance of hit characterization experiments by another operator (for hit compounds GSK 1210151A, Ro 11-1464, vorinostat and TSA) further confirmed data reproducibility (Fig.3-16 - Fig.3-18).

During HTS of LOPAC ${ }^{\circledR 1280}$ and ENZO ${ }^{\circledR 774}$ libraries, identification of inhibitory-acting compounds was restricted due to the high signal variability of the solvent control condition (chapter 3.3.1, Fig.3-12, Fig.3-13). Identification of true hit compounds that modulate endogenous CEBPD was further limited by the restricted nature of the used CEBPD promoter, which cannot integrate or respond to all regulatory signals. Hit compounds trichostatin A and vorinostat displayed opposite effects on expression of the reporter CEBPD::SEAP and the endogenous CEBPD genes (chapter 3.4, Fig.3-18). However, knowing differences in promoter structure enables identification of regulatory sequences 
that potentially explain the observed effects of CEBPD transcription in target cells.

The generated screening assay was selective to BET and HDAC inhibitors - the pharmacological classes of hit compounds. There are no other BET inhibitors besides GSK $1210151 \mathrm{~A}$ and Ro 11-1464 among epigenetically active compounds of LOPAC $^{\circledR 1280}$ library. Among epigenetically active compounds in ENZO ${ }^{\circledR 774}$ library, sodium valproate also acts as class I and IIa HDAC inhibitor. ${ }^{227}$ However, sodium valproate showed no prominent effect on SEAP secretion during HTS, in contrast to TSA and vorinostat, presumably due to its selective and context-specific action on expression of inflammatory genes. ${ }^{228}$

In summary, the generated multi-gene-reporter cassette 1.0 is a flexible, reliable, and sensitive screening instrument. The developed screening assay was sensitive and specific identifying CEBPD-activatory compounds. A reliable detection of CEBPD-inhibitory compounds was impaired, due to high variability of solvent control condition. Identification of true hit compounds required additional experimental conformation, as the multi gene-reporter cassette 1.0 was expressed under control of the simplified CEBPD promoter. The developed screening assay displays a potential for drug discovery and

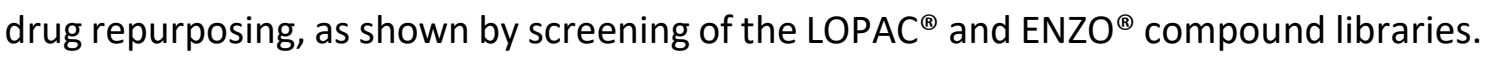

\subsection{Characterization of CEBPD-modulating hit compounds}

\subsubsection{Hit compounds and a general principle of their action}

Characterisation of hit compound-mediated pharmacological activity requires a short display of the basic principle of epigenetic gene transcription control by lysine acetylation. Acetylation and deacetylation of lysine residues on histones and non-histone regulatory proteins are important epigenetic mechanisms of gene transcription regulation in eukaryotic cells. ${ }^{229-231}$ Mutual action of proteins, which can add ("writers"), recognize ("readers"), and remove ("erasers") protein acetylation marks underscore epigenetic gene expression control. ${ }^{229}$

Histone acetyltransferases - HATs - act as writer proteins adding acetyl groups to the lysine residues on histones and non-histone regulatory proteins, $228,229,231$ including C/EBPS. ${ }^{232}$ In nucleosome, lysine acetylation in amino-terminal tails of core histones 
alters their net protein charge resulting in their weaker interaction with DNA and thus formation of euchromatin - a precondition for activation of gene expression 228,233 (Fig.4-1A). Acetylation of non-histone regulatory proteins like transcription factors leads to their transcriptional activation and enhanced DNA binding (Fig.4-1A).

Acetylated lysine residues serve as recognition signals for bromodomain-containing proteins (BCPs) functioning as epigenetic reader proteins. ${ }^{229}$ Bromodomain (BRD) is an evolutionarily conserved protein interaction module that forms a hydrophobic lysine acetylation binding site. ${ }^{234}$ Multiple (at least two) amino-terminal BRDs occur in tandem organisation in bromodomain and extra-terminal domain (BET) proteins including BRD2, BRD3, BRD4, and BRDT. ${ }^{234,235}$ Being able to recognise and bind $\varepsilon-\mathrm{N}$-acetylation of lysine residues in histones and non-histone proteins ${ }^{234,236}$ (Fig.4-1A), BCPs can regulate gene transcription as follows: i) by acting as scaffolds for recruitment and binding of large transcriptional protein complexes ${ }^{235}$ or self-regulated transcription factors ${ }^{233}$; ii) by acting as methyltransferases, ATP-depending chromatin remodelling complexes, HATs or helicases ${ }^{237}$; iii) by binding and targeting of acetylated transcription factors to particular DNA binding sites. 238,239

In contrary to HATs, histone deacetylases (HDACs) remove acetyl groups from protein Iysine residues functioning as epigenetic eraser proteins. ${ }^{228,229}$ Human 18 HDAC enzymes are classified into classes I-IV according to the homology in their catalytic cores ${ }^{240-242}$ : i) zinc-dependent HDACs of class la (HDAC1, HDAC2), class lb (HDAC3), and class Ic(HDAC8) ${ }^{243}$; ii) zinc-dependent HDACs of class Ila (HDAC4, HDAC5, HDAC7, and HDAC9) and class IIb (HDAC6, HDAC10) 241,242 ; iii) NAD-dependent ${ }^{240}$ class III HDACs ${ }^{243}$ - sirtuins; iv) class IV HDAC (HDAC11). ${ }^{244}$ Despite the nomenclature, HDACs can deacetylate also non-histone proteins ${ }^{229}$ (Fig.4-1A). Over 50 non-histone regulatory proteins belong to the HDAC targets including transcription factors (e.g. NF-kB 245 and STAT3 ${ }^{246}$ ), transcription regulators, signal transduction mediators, DNA repair enzymes, nuclear import regulators, and inflammation mediator proteins. ${ }^{242,246-248}$ Also CEBPD promoter contains experimentally confirmed binding sites of HDAC1, 2, and 6. ${ }^{4 *}$ HDACs can repress gene transcription by: i) histone deacetylation resulting in formation of heterochromatin unat-

\footnotetext{
4* CEBPD gene, ENCODE transcription factor targets. Harmonizome internet site. http://amp.pharm.mssm.edu/Harmonizome/gene/CEBPD. Accessed April 26, 2020.
} 
A

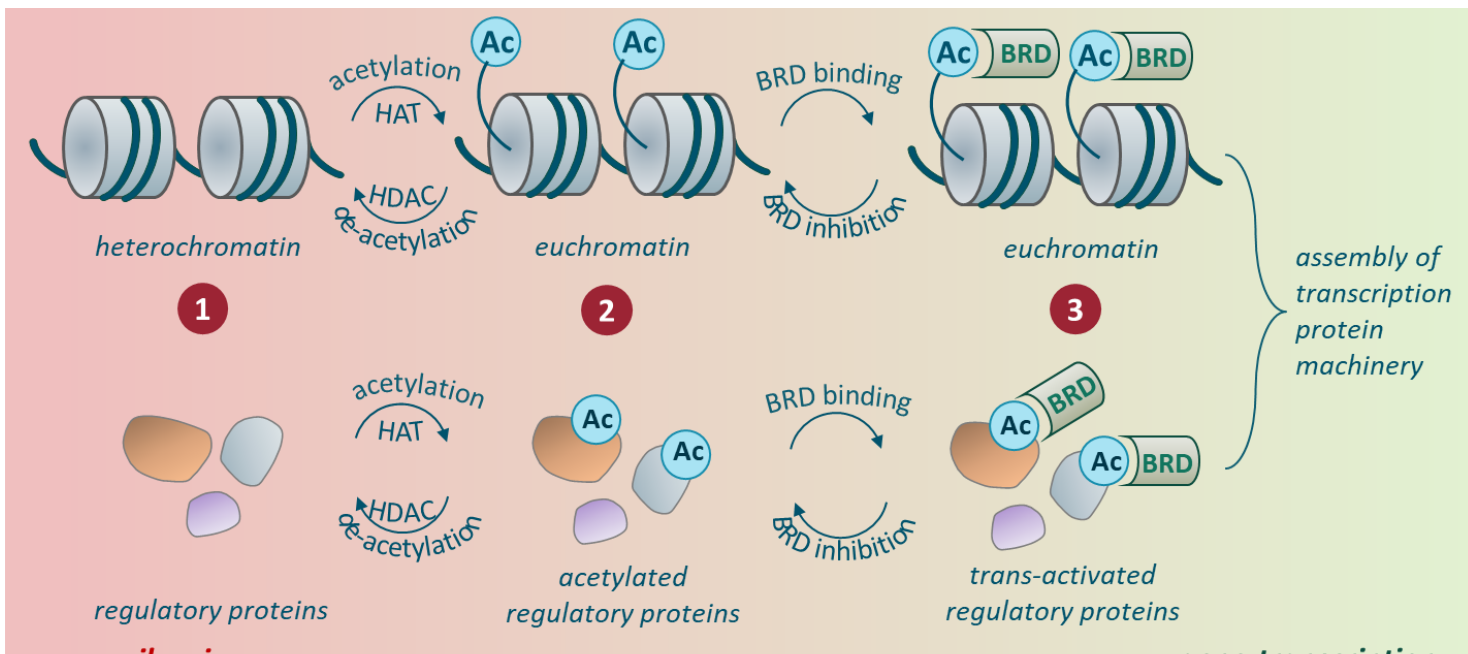
gene silencing

B

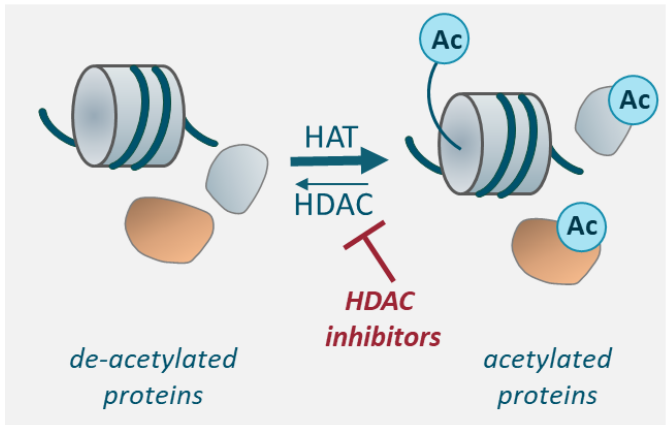

C

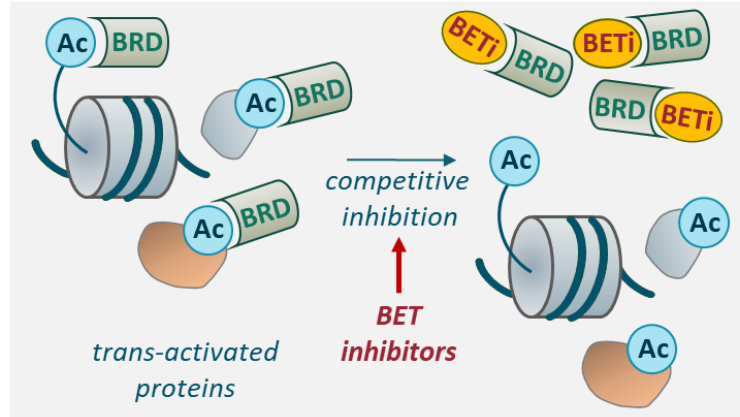

Figure 4-1: Epigenetic regulation of gene expression by protein acetylation as well as by action of HDAC and BET inhibitors. A: The non-modified heterochromatin displays a closed chromatin structure that cannot be assessed by transcription protein machinery (1). Further, the non-modified regulatory proteins do not display the necessary transcriptional activity (1). Acting as "writer" proteins, HATs acetylate N-terminal lysine residues of histones resulting in euchromatin formation that shows an open chromatin structure assessable for transcriptional machinery (2). The acetylation of regulatory proteins enables their transcriptional activation and DNA binding (2). Acting as "reader" proteins, BRD-containing BCPs recognize and target acetylated histones and non-histone proteins leading to the assembly of transcription protein machinery and trans-activation of regulatory proteins (3). Acting as "eraser" proteins, HDACs deacetylate histones and trans-activated regulatory proteins promoting silencing of gene transcription (1). B: Environment-responsive HDAC inhibitors target acetylated histones and non-histone proteins generating protein hyperacetylation state that can result in activated or inhibited gene transcription. C: BET inhibitors competitively inhibit BCP binding to acetyl residues on histones and trans-activated regulatory proteins causing up- or downregulation of gene expression.

tainable for transcription machinery ${ }^{228}$; ii) inhibition of trans-activated transcription factors. 245,246

A balanced action of HATs and HDACs is disturbed by HDAC inhibitors (HDACi), which bind to a zinc ion in the catalytic enzyme domain and block HDAC enzymatic activity 249 leading to a hyper-acetylation of histones and a sustained acetylation of non-histone regulatory proteins ${ }^{249,250}$ (Fig.4-1B). HDACi are small molecules that cluster according to their chemical structure into five classes: i) hydroxamic acids (e.g. trichostatin A, vori- 
nostat); ii) short chain aliphatic acids; iii) benzamides; iv) cyclic tetrapeptides; v) sirtuin inhibitors. ${ }^{251}$ According to their action, HDACi affect gene transcription by: i) disturbance of acetylation-related protein function, protein stability, or protein-protein interacttions $^{246}$; ii) mediation of incorrectly positioning that misdirects reader proteins ${ }^{252,253}$; iii) induction of genes that inhibit expression of others (negative feedback). ${ }^{252,253}$ The genome-wide studies revealed that HDACi induce a number of genes approximately equal to those they repress. ${ }^{228,229}$

$B E T$ inhibitors (BETi) also selectively alter gene transcription that can result in up- or downregulation of gene expression. ${ }^{228} \mathrm{BETi}$ are small molecules that cluster into two major classes according to their action - these that do or do not mimic acetylated lysines. ${ }^{254}$ To the acetylated lysine mimetics belong thienotriazoldiazepines (e.g. (+)-JQ1 52, Ro 11-1464), isoxazoles (e.g. GSK 1210151A), benzodiazepines, and benzotriazepines. ${ }^{254}$ BETi bind acetylated histones competitively (Fig.4-1C) displacing transcription protein complexes from chromatin and therefore preventing transcription of BCP's target genes. ${ }^{233} \mathrm{BETi}$ also competitive inhibit bromodomain binding of acetylated regulatory proteins (Fig.4-1C) like transcription factors (e.g. NF-kB subunit p65) causing altered gene expression of corresponding target genes.

The four CEBPD-modulating hit compounds, discovered in this study, belong to BETi and HDACi classes.

GSK 1210151A (Fig.4-2A) is a patented ${ }^{5 *}$ compound of Glaxo Smith Kline Inc. (UK), discovered in 2012. GSK 1210151A (alternative names GSK-151, I-BET151, iBET-151) acts as a potent apoA1 activator ${ }^{255}$ and selective BRD2, BRD3, and BRD4 BET inhibitor displaying very strong binding mode ( $\mathrm{K}_{\mathrm{d}}$ of 50-100 nM). ${ }^{256,257}$ In GSK $1210151 \mathrm{~A}$ molecule, isoxazole heteroatoms are responsible for binding acetylated lysine recognition pocket on BRDs. ${ }^{256,258,259}$ GSK $1210151 \mathrm{~A}$ acts anti-inflammatory by regulation of pro-inflammatory cytokine and chemokine production in LPS-activated $M \varphi^{260}$ and by transcription control of inflammation-linked genes in various cell types. ${ }^{261}$ The anti-inflammatory action of GSK $1210151 \mathrm{~A}$ also represents a potential therapeutic strategy of inflammatory

\footnotetext{
5* Integrity, A Cortellis solution. Clarivate analytics website. Search term: GSK 1210151A https://integrity.clarivate.com. Accessed February 20, 2020.
} 
A

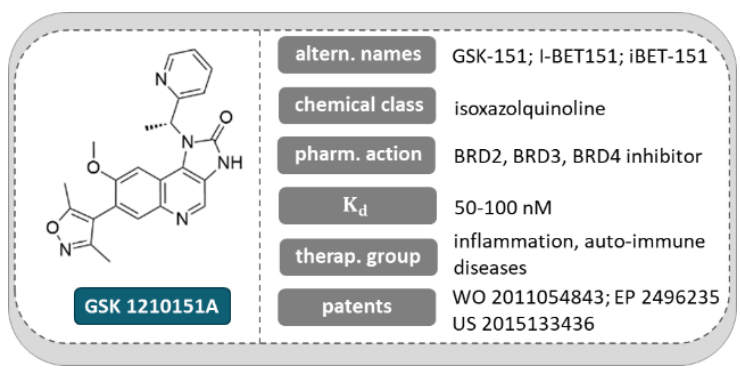

C

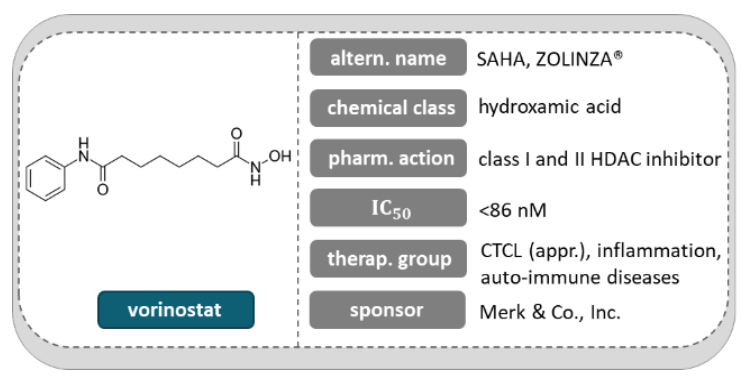

B

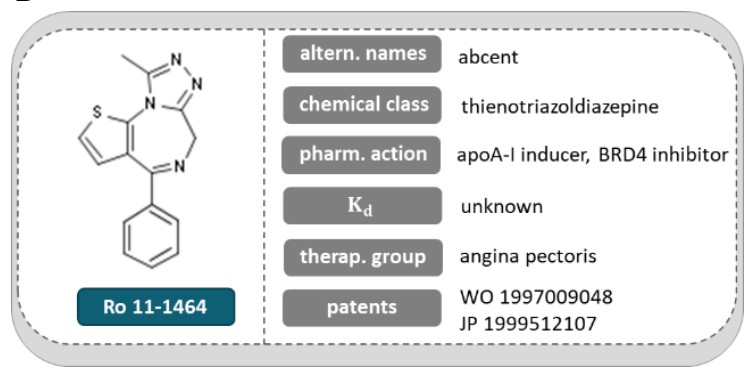

D

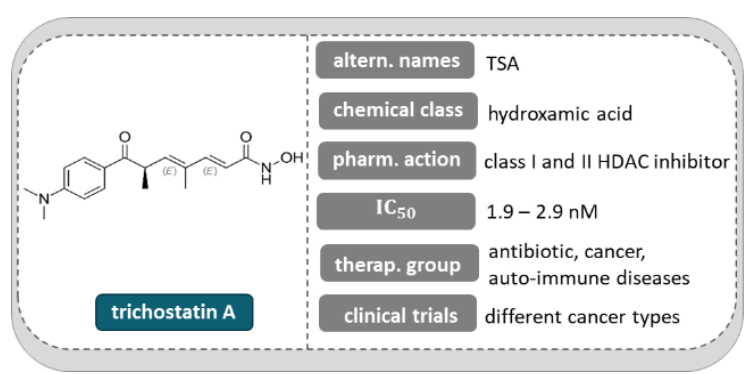

Figure 4-2: Key characteristics of the confirmed hit compounds. Chemical structure, alternative (altern.) names, chemical classes, pharmacological (pharm.) action, half maximal inhibitory concentration $\left(\mathrm{IC}_{50}\right)$ or dissociation constant, therapeutic group, and sponsor of GSK 1210151A (A), Ro 11-1464 (B), vorinostat (C), and trichostatin A (D).

diseases including RA. ${ }^{233}$

Ro 11-1464 (Fig. 4-2B) is a patent-protected ${ }^{6 *}$ compound of F. Hoffmann-La Roche AG (Switzerland) that is first reported to stimulate production of high-density lipid protein apoA-I in cultured liver cells Hep G2. ${ }^{262}$ Sharing structural similarities with (+)-JQ1, a potent BET inhibitor, ${ }^{7}$ Ro 11-1464 also binds BETs, displays, however, weak inhibition potential. ${ }^{263}$ Analysis of the thermal shift measurements of human BET-BRDs in the presence of $10 \mu \mathrm{M}$ Ro 11-1464 revealed only weakly binding of BRD4 as well as absent binding of BRD2 and BRD3 in comparison to this of (+)-JQ1. ${ }^{263} \mathrm{Up}$ to date, known therapeutic potential of Ro 11-1464 is mostly based on its action as a potent apoA-I inducer in liver cells as a potential anti-atherosclerotic agent. ${ }^{264}$

Discovered as an improved hybrid polar compound in 1996, ${ }^{265}$ vorinostat (Fig.4-2C) or suberoylanilide hydroxamic acid (SAHA) is a synthetic compound that is approved by FDA for the treatment of cutaneous T-cell lymphoma (CTCL) in USA in 2006. ${ }^{266}$ Vorinostat is a potent HDAC inhibitor $\left(\mathrm{IC}_{50}<86 \mathrm{nM}^{7 *}\right.$ ) for class I (HDAC1-3, and 8) and class

\footnotetext{
${ }^{6 *}$ Integrity, A Cortellis solution. Clarivate analytics website. Search term: Ro 11-1464 https://integrity.clarivate.com. Accessed February 20, 2020.

${ }^{7 *}$ Zolinza highlights of prescription information. Merck website.
} 
II (HDAC4-7, and 10) HDACs that also displays anti-inflammatory properties detected in human primary $\mathrm{M} \varphi^{267,268}$ and in rodent models of inflammation. ${ }^{267,268}$ The anti-inflammatory action of vorinostat is considered as a potential treatment strategy of inflammatory diseases like RA. ${ }^{269}$

Discovered during screening for antifungal antibiotics in $1973,{ }^{270}$ trichostatin A (TSA) (Fig.4-2D) is a natural compound from Streptomyces spp., which displays both antifungal antibiotic and HDAC inhibitory activity in mammalian cells. ${ }^{249}$ TSA functions as a potent class I (HDAC1-3, and 8) and class II (HDAC4-7, and 10) HDAC inhibitor ${ }^{271}$ with IC $_{50}$ ranging between 1.9 and $2.9 \mathrm{nM}$ for purified HDACs. ${ }^{272}$ TSA causes altered expression of various genes in the context of inflammation ${ }^{250}$ and cancer. ${ }^{251}$ Despite its potential anticancer action (phase I and II clinical trial for leukemia, lymphoma, and myeloma), ${ }^{230}$ TSA reduces inflammation and represents a potential treatment strategy for RA. ${ }^{273}$

\subsubsection{Hit compounds affect gene expression in THP-1 M $\varphi$}

Gene expression of reporter CEBEPD::SEAP, endogenous CEBPD, IL-6, IL-1ß, and CCL2 genes was significantly induced by M1 treatment, at the 4 hour time point (Fig.3-16A, Fig.3-17A, Fig.3-18A, 4h, blue bars). However, M1 treatment displayed different effects on gene expression of endogenous CEBPD and reporter CEBPD::SEAP genes at the later time point. Gene expression of the endogenous CEBPD decayed over time and failed to be statistically significant relative to MO control, at the 24 hour time point (Fig.3-16A, Fig.3-17A, Fig.3-18A, CEBPD, 24h, blue bars). In contrary, gene expression of the reporter CEBPD::SEAP induced by M1 treatment to a similar extent to the endogenous CEBPD, reduced less and still displayed a significant upregulation at the later time point (Fig.3-16A, Fig.3-17A, Fig.3-18A, CEBPD::SEAP, 24h, blue bars).

All four hit compounds significantly upregulated gene expression of reporter CEBPD::SEAP to a similar extent, at the 4 hour time point (Fig.3-16A, Fig.3-17A, Fig.3-18A, CEBPD::SEAP, 4h, red and orange bars; Table 3.4, 3.5, 3.6). At the 24 hour time point, reporter CEBPD::SEAP gene expression was either almost unchanged by TSA

https://www.accessdata.fda.gov/drugsatfda_docs/lael/2011/021991s 002lbl.pdf. Accessed February 20, 2020. 
and Ro 11-1464 or elevated by GSK 1210151A and vorinostat (Fig.3-16A, Fig.3-17A, Fig.3-18A, CEBPD::SEAP, 24h, red and orange bars; Table 3.4, 3.5, 3.6). However, gene expression of the endogenous CEBPD was upregulated only by BETi, to a similar extend like the reporter CEBPD::SEAP at the early time point (Fig.3-16A, Fig.3-17A, CEBPD, 4h, red bars; Table 3.4, 3.5). Surprisingly, HDACi completely abolished the M1 treatmentactivated gene expression of endogenous CEBPD at both time points tested (Fig.3-18A, CEBPD, 4h, 24h, red and orange bars; Table 3.6).

The opposed effect of BETi and HDACi on gene expression of endogenous CEBPD was unexpected, as both inhibitor classes are known for their anti-inflammatory action. Remarkably, both BETi and HDACi are epigenetically active compounds that directly regulate gene transcription ( $c f$. chapter 4.2.1, Fig.4-1B, C). Presumably, HDACi coincidently display an activatory cis- and a larger inhibitory trans-effect. Whereas activatory cis-effect (e.g. forcing of the proximal CEBPD promoter accessibility to a transcriptional machinery) may result in upregulated expression of both endogenous CEBPD and reporter CEBPD::SEAP genes, inhibitory trans-effect, however, may affect only the distal endogenous CEBPD promoter and may dominate over the cis-activation. In contrary, BETi may act exclusively through the control of the cis-activatory mechanisms. As the defined CEBPD promoter contains only the most proximal CREB, SP1, STAT3, and presumably NF-kB TF binding sites (Fig.1-3A, B), BETi can activate the corresponding signalling pathways leading to the elevated endogenous CEBPD and reporter CEBPD::SEAP gene expression (Fig.4-3, Fig.4-4). The defined CEBPD promoter may express a disrupted (Fig.1-3, Fig.8-2) and therefore non-functional ATF3 binding site that is important for the inhibition of endogenous CEBPD gene transcription in $M \varphi^{71}$ (Fig.4-3, Fig.4-4).

The NF-kB-ATF3 regulatory circuit, accompanied by the CEBPD auto-regulation, belongs to one of the prominent regulatory mechanisms of CEBPD gene transcription in LPStreated $\mathrm{M} \varphi^{71}$ (cf. chapter 1.3.2.1). Induced by LPS-activated NF-kB and self-promoter binding, CEBPD gene expression is inhibited by ATF3 that directly binds CEBPD promoter 3-4 hours after LPS-treatment. ${ }^{71}$ As the expression of reporter CEBPD::SEAP displayed a significant increase even one day after M1 treatment, it may not be inhibited by ATF3. The defined CEBPD promoter may display no functional ATF3 binding site, while the endogenous CEBPD promoter contains a functional ATF3 binding site that locates more 
distal (> $300 \mathrm{bp}$ ) relative to TSS in human THP-1 M $\varphi$. Alternatively, inhibition of CEBPD promoter activity may happen in ATF3-independent way.

The opposing effects of BETi and HDACi on gene expression of the endogenous CEBPD raise a question about their effects on gene expression in the downstream signal cascade. Hit compound-mediated expression of pro-inflammatory marker-genes IL-6, IL-1ß, which belong to the direct targets of C/EBPS in $M \varphi,{ }^{71}$ and CCL2 was analysed. BETi and HDACi both significantly suppressed the M1 treatment-activated gene expression of IL-6 and CCL2 to a similar extend, at both time points tested (Fig.3-16A, Fig.3-17A, Fig.3-18A, IL-6, CCL2, 4h, 24h, red, orange bars; Table 3.4, 3.5, 3.6). Surprisingly, BETi and HDACi displayed differing effects on IL-1ß gene expression. At the early time point, Ro 11-1464 showed no significant effect, while GSK 1210151A significantly and clearly upregulated IL-1ß gene expression that was inhibited by both BETi at the late time point (Fig.3-16A, Fig.3-17A, IL-1ß, 4h, 24h, red bars; Table 3.4, 3.5). HDACi showed only a minor significant upregulation of IL-1ß gene expression at the 4 hour time point, which elapsed at the 24 hour time point (Fig.3-18A, IL-1ß, 4h, 24h, red, orange bars; Table 3.6).

BETi-upregulated CEBPD may contribute to the elevated expression of IL-1ß. C/EBPS TF can build via its bZIP domain homotypic heterodimers with members of the CREB/ATF family ${ }^{274}$ recognizing the composed half C/EBPS and half CREB/ATF DNA binding sites. ${ }^{74}$ Two distinct C/EBP-CRE/ATF heterodimers bind an asymmetrical C/EBP-CRE site in the human IL-1 $\beta$ gene enhancer in response to LPS treatment. ${ }^{275} \mathrm{C} /$ EBP $\delta$ TF can also dimerize with AP-1 proteins including c-foc and c-jun and bind composite binding sites, which are preserved in over 40 human and mouse promoters ${ }^{36}$ including IL-1ß. ${ }^{275}$

C/EBPS TF can also interact (via largely unknown domains) with NF-kB family members, even though no proper C/EBPS-NF-kB heterodimer and corresponding composite DNA binding site are known. ${ }^{36}$ The heterodimeric C/EBP-NF-kB complexes can bind both, C/EBP ${ }^{276}$ and NF-kB DNA binding sites. ${ }^{277}$ Thus, C/EBP $\delta$ TF may trans-activate genes that lack direct C/EBP binding sites and therefore display a larger set of target genes than assumed. ${ }^{36}$ Functional, and often synergistic, C/EBP-NF-kB interactions regulate transcription of various genes ${ }^{278}$ that display overlapped or neighbour C/EBP and NF-kB binding sites. ${ }^{279,280}$ So, C/EBPs and NF-kB synergistically regulate expression of IL-6 and IL-8 genes in $\mathrm{M} \varphi .^{281}$ Such synergistic transcription factor activity is presumably high- 
lighted by the compliant binding or synergistic recruiting of basal transcription machinery proteins and co-activators. ${ }^{282}$

GSK 1210151A

BET inhibitor GSK 1210151 suppressed the M1 treatment-induced gene expression of IL-6, IL- $ß$ and CCL2 in THP-1 reporter M $\varphi$. To my best knowledge, this study is the first evidence for GSK 1210151A affecting gene expression of CEBPD and CCL2. The anti-inflammatory effect of GSK $1210151 \mathrm{~A}$ is consistent with the literature, as the compound inhibits cytokine secretion in RASF ${ }^{261}$ and inflammatory gene expression in RAW264.7283 and PBMCs ${ }^{255,284}$ (Table 4.1).

GSK 1210151A may control transcription of IL-6 by regulation of BRD4-dependent binding of CREB-binding protein (CBP) transcriptional co-regulator to the IL-6 promoter NF-kB pathway (Fig.4-3). The LPS-responsive transcription factor NF-kB, which is known

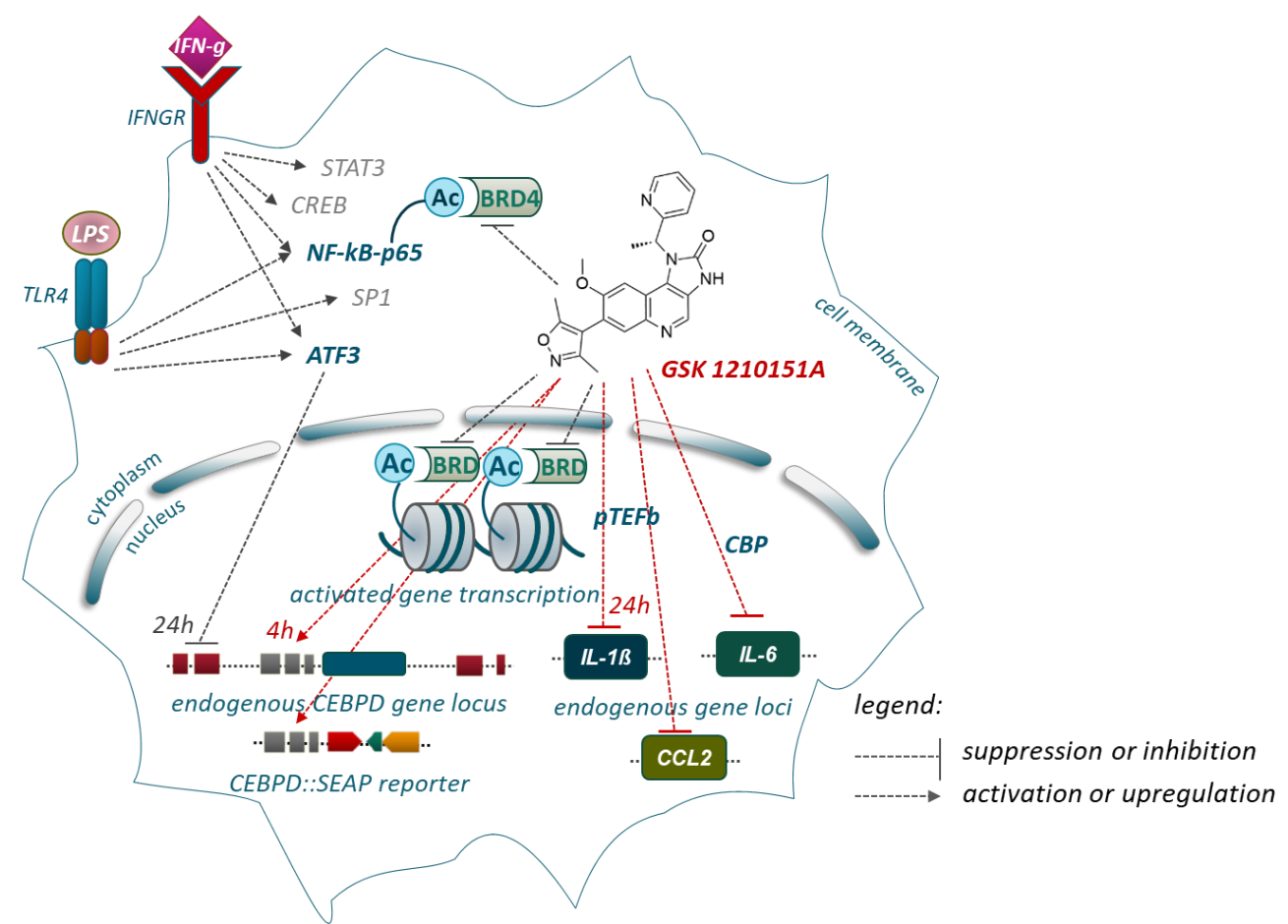

Figure 4-3: The proposed pharmacological activity of BET inhibitor GSK 1210151A in M1-polarized THP1 reporter M $\varphi$. GSK 1210151A (red lines) repressed M1 treatment-activated gene expression of IL-6 and CCL2 and upregulated CEBPD::SEAP expression at both time points tested. However, GSK 1210151A showed its activatory effect on the endogenous CEBPD gene expression and suppressive for IL-1ß, at indicated time points. GSK 1210151A may control cis-activatory mechanisms resulting in upregulation of reporter CEBPD::SEAP and endogenous CEBPD gene expression. As the defined CEBPD promoter may lack functional ATF3 regulatory site, the activated expression of CEBPD::SEAP cannot be inhibited like that of endogenous CEBPD at the later time point. GSK 1210151A may downregulate gene expression of IL-6 by preventing of CBP transcriptional co-regulator binding to the IL-6 promoter. Further, GSK 1210151A may suppress recruitment of pTEFb leading to the inhibited gene expression of IL-1ß and CCL2, which may be also supressed epigenetically by GSK 1210151A-mediated inhibition of BRD4. For details please refer to the main text. 
Table 4.1: Literature reported effects of hit compounds on gene expression and cytokine secretion.

\begin{tabular}{|c|c|c|c|c|c|c|c|}
\hline compound & $\begin{array}{l}\text { concen- } \\
\text { tration }\end{array}$ & $\begin{array}{l}\text { organism/ } \\
\text { tissue/ cell } \\
\text { type }\end{array}$ & $\begin{array}{l}\text { stimu- } \\
\text { lus }\end{array}$ & gene & readout & effect & ref. \\
\hline \multirow{5}{*}{ GSK 1210151A } & & \multirow{2}{*}{ RASF } & basal & TNF- $\alpha$ & \multirow{2}{*}{$\begin{array}{l}\text { protein } \\
\text { secretion }\end{array}$} & \multirow{2}{*}{ inhibition } & \multirow{2}{*}{261} \\
\hline & & & IL1-ß & IL-6 & & & \\
\hline & & RAW264.7 & LPS & IL-6 & mRNA & inhibition & 283 \\
\hline & & PBMC & LPS & IL-6 & mRNA & inhibition & $\begin{array}{l}255, \\
284\end{array}$ \\
\hline & & RAW264.7 & & IL-1ß & mRNA & inhibition & 283 \\
\hline \multirow{10}{*}{ vorinostat } & \multirow[t]{2}{*}{$30 \mu \mathrm{M}$} & \multirow{2}{*}{$\begin{array}{l}\text { GM-CSF- } \\
\text { stimulated } \\
\text { HMDM }\end{array}$} & \multirow[b]{2}{*}{ LPS } & IL-6 & \multirow{2}{*}{$\begin{array}{l}\text { protein } \\
\text { secretion }\end{array}$} & reduction & \multirow[b]{2}{*}{268} \\
\hline & & & & $\mathrm{IL}-1 ß$ & & $\begin{array}{l}\text { upregula- } \\
\text { tion }\end{array}$ & \\
\hline & \multirow{2}{*}{$\begin{array}{c}0.1 \text { - } 50 \\
\mathrm{mg} / \mathrm{kg} \\
\text { (orally) }\end{array}$} & \multirow{2}{*}{$\begin{array}{l}\text { BALB/c } \\
\text { mouse }\end{array}$} & \multirow{2}{*}{ LPS } & IL1-ß & \multirow{2}{*}{$\begin{array}{l}\text { protein } \\
\text { secretion }\end{array}$} & \multirow{2}{*}{ reduction } & \multirow{2}{*}{267} \\
\hline & & & & IL-6 & & & \\
\hline & $\begin{array}{l}\text { low dose } \\
(<3 \mu \mathrm{M})\end{array}$ & \multirow{2}{*}{$\begin{array}{c}\text { M-CSF- } \\
\text { stimulated } \\
\text { HMDM }\end{array}$} & \multirow{2}{*}{ LPS } & IL-6 & \multirow{2}{*}{$\begin{array}{l}\text { protein } \\
\text { secretion }\end{array}$} & reduction & \multirow{2}{*}{268} \\
\hline & $\begin{array}{l}\text { high dose } \\
(>3 \mu \mathrm{M})\end{array}$ & & & IL1-ß & & $\begin{array}{l}\text { upregula- } \\
\text { tion }\end{array}$ & \\
\hline & $\begin{array}{l}\text { low dose } \\
(1 \mathrm{mg} / \mathrm{kg})\end{array}$ & \multirow{2}{*}{ CIA-rat } & \multirow[b]{2}{*}{-} & \multirow{2}{*}{-} & \multirow{2}{*}{-} & $\begin{array}{l}\text { anti-in- } \\
\text { flamma- } \\
\text { tory }\end{array}$ & \multirow{2}{*}{268} \\
\hline & $\begin{array}{l}\text { high dose } \\
(5 \mathrm{mg} / \mathrm{kg})\end{array}$ & & & & & $\begin{array}{l}\text { pro-in- } \\
\text { flamma- } \\
\text { tory }\end{array}$ & \\
\hline & $\begin{array}{c}\text { low dose } \\
(0.1-0.2 \\
n M)\end{array}$ & THP-1 & LPS & IL-6 & $\begin{array}{l}\text { protein } \\
\text { secretion }\end{array}$ & reduction & 299 \\
\hline & & PBMC & LPS & IL-1ß & mRNA & no effect & 267 \\
\hline \multirow{7}{*}{ trichostatin A } & $\begin{array}{l}12.5,25 \\
n M(6 h)\end{array}$ & \multirow{4}{*}{ mBMDM } & \multirow{4}{*}{ LPS } & \multirow{2}{*}{$\mathrm{IL}-1 ß$} & mRNA & no effect & \multirow{4}{*}{305} \\
\hline & $25 \mathrm{nM}(\mathrm{h})$ & & & & mRNA & reduction & \\
\hline & \multirow{2}{*}{$\begin{array}{c}12.5-25 \\
\mathrm{nM}\end{array}$} & & & \multirow[b]{2}{*}{ IL-6 } & mRNA & reduction & \\
\hline & & & & & $\begin{array}{l}\text { protein } \\
\text { secretion }\end{array}$ & reduction & \\
\hline & & \multirow{2}{*}{ mBMDM } & LPS & & & & \\
\hline & & & basal & CCL2 & mRNA & reduction & 306 \\
\hline & & RAW264.7 & LPS & cebpd & mRNA & $\begin{array}{l}\text { abolish- } \\
\text { ment }\end{array}$ & 309 \\
\hline
\end{tabular}


to be sensitive to acetylation and to be targeted by $\mathrm{BRD} 4,233$ activates pro-inflammatory acetylated p65 (NF-kB subunit) in RAW264.7 murine $M \varphi$, although it blocks associative gene transcription including IL-6. ${ }^{71}$ However, GSK $1210151 \mathrm{~A}$ has no effect on acetylation, phosphorylation, nuclear translocation or DNA binding of transcriptionally active of BRD4 with NF-kB-p65. ${ }^{283}$ Instead, GSK 1210121 A possibly prevents the binding of CBP to the IL- 6 promoter resulting in reduced IL- 6 expression, as seen in LPS-treated RAW264.7.283

The observed suppression of IL-1ß and CCL2 gene expression may result from GSK 1210151A-inhibited activity of corresponding TLR4 or IFN-g signalling pathways (Fig.4-3). As introduced previously, LPS and IFN-g induce a battery of genes including NF-kB, ATF3, CREB, SP1, and STAT3 through the activation of TLR4 and JAK-STAT signalling pathways, which are characteristic of polarization and inflammatory responses of $\mathrm{M} \varphi$ (cf. chapter 1.3.2.1). According to their predominant mechanism of action, BETi supress recruitment of positive transcription elongation factor $b$ (pTEFb) resulting in reduced transcription elongation and therefore inhibited gene expression. ${ }^{285}$ In human monocytes, GSK $1210151 \mathrm{~A}$ repress transcription of STAT target genes in response to cytokine (e.g. IFN-g) treatment, as well as TLR4-induced IFN responses, mainly at the transcription initiation level. ${ }^{285}$ Such intervention of GSK $1210151 \mathrm{~A}$ in TLR4 and INF-g signalling may also occur in THP-1 M $\varphi$, however, not affecting the activity of CEBPD promoter, as gene expression of the endogenous CEBPD and the reporter CEBPD::SEAP was upregulated by GSK 1210151 A. Alternatively, GSK 1210151A may epigenetically repress expression of CCL2 by inhibition of BRD4, as shown for other BET inhibitors IBET and JQ1 in activated $\mathrm{M} \varphi .^{260,286}$

\section{Ro 11-1464}

BET inhibitor Ro 11-1464 suppressed the M1 treatment-activated gene expression of IL-6, IL-1ß, and CCL2 in THP-1 reporter M $\varphi$. To my best knowledge, this study is the first report of Ro 11-1464-mediated gene expression regulation in general and its corresponsive anti-inflammatory action in THP-1 M $\varphi$. The pharmacological action Ro 11-1464 as both BET inhibitor and apoA-I inducer may underline its observed effects on gene expression in target cells. 
Ro 11-1464 may suppress gene expression of IL-1ß, IL-6, and CCL2 through the BRD4 inhibition $^{263}$ (Fig.4-4). BRD4, which is the most-studied member of the BET protein family, ${ }^{233}$ regulates gene transcription by following mechanisms: i) by direct assembly of HAT-dependent chromatin complexes resulting in recruitment of $\mathrm{pTEFb}^{287,288}$ and by RNA polymerase II phosphorylation ${ }^{289}$ activating gene transcription; ii) by binding of p65 NF-kB subunit acetylated Lys310 residue regulating transcriptional activity of NF-kB ${ }^{233}$; iii) by self-recruitment to enhancers ${ }^{290}$ and assessment of transcriptional elongation. ${ }^{291}$

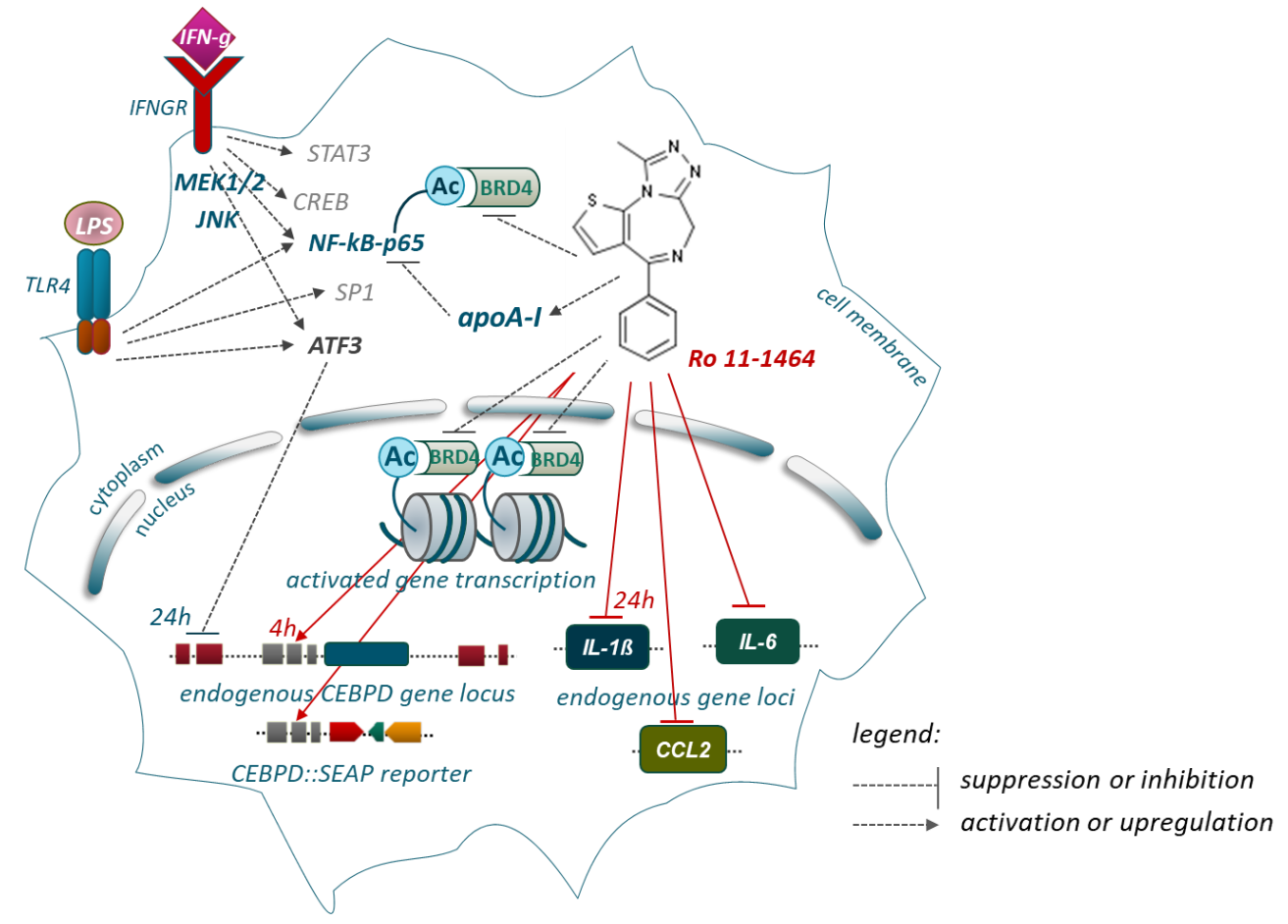

Figure 4-4: The proposed pharmacological activity of BET inhibitor Ro 11-1464 in M1-polarized THP-1 reporter M $\boldsymbol{\varphi}$. Ro 11-1464 (red lines) repressed M1 treatment-activated gene expression of IL-6 and CCL2 and upregulated that of CEBPD::SEAP at both time points tested. However, Ro 11-1464 showed its activatory effect on gene expression of the endogenous CEBPD and suppressive for IL-1ß, at indicated time points. Ro 11-1464 may control cis-activatory mechanisms resulting in upregulation of reporter CEBPD::SEAP and endogenous CEBPD gene expression. As the defined CEBPD promoter may lack functional ATF3 regulatory site, the activated expression of CEBPD::SEAP cannot be inhibited like that of endogenous CEBPD at the later time point. Ro 11-1464 may downregulate gene expression by its anti-inflammatory action as a relatively weak BRD4 inhibitor or a potent apoA-I activator. The Ro 11-1464-activated apoA-I, potentially via JNK or MEK1/2 signalling pathways, may selectively damp TLR4 signalling or reduce nuclear NF-kB-p65 subunit resulting in repressed expression of IL-6, IL-1ß and CCL2. For details please refer to the main text.

The interaction disruption between BRD4 and Ac-p65 by BET inhibitor (+)-JQ1 results in degradation of constitutively active nuclear p65 and thus NF-kB target gene expression alteration. ${ }^{292}$ Individual silencing of BRD4 resulted in reduced secretion of pro-inflammatory IL-1ß, IL-6, and TNF- $\alpha$ cytokines in LPS-stimulated human $M \varphi .^{260,293}$ Ro 11-1464, however, binds BRD4 weakly and therefore shows low BET inhibitory potential. ${ }^{263}$ 
Alternatively, Ro 11-1464 may suppress gene expression of IL-1ß, IL-6, and CCL2 by upregulation of apoA-I, a well-known anti-inflammatory agent, ${ }^{294}$ and the apoA-I-mediated blunting of TLR4 and INF-g signalling pathways in target cells (Fig.4-4). ApoA-I is a major protein subunit of high-density lipoprotein (HDL) that regulates cholesterol transfer, inflammatory and immune responses ${ }^{294}$ and is also expressed in human PBMC- and THP-1 monocytes and M $\varphi .{ }^{295-297}$ Induction of apoA-I involves JNK and MEK1/2 signalling pathways, while inhibition of NF-kB, JNK, or p38 obstructs the elevation of apoA-I. ${ }^{297}$ Endogenous apoA-I decreases expression of LPS-induced pro-inflammatory genes including TNF- $\alpha$ and IL-1ß by a selective dampening of TLR4 signalling in M $\varphi .{ }^{295}$ Additionally, in pre-treated human primary monocytes, apoA-I decreases CCL2 mRNA, CCL2 protein synthesis and secretion levels in dose-dependent manner by reduction of nuclear NF-kB-p65 subunit. ${ }^{298}$

\section{Vorinostat}

HDAC inhibitor vorinostat had the following effects on the M1 treatment-induced gene expression in THP-1 reporter $M \varphi$ : complete abolishment of the endogenous CEBPD, suppression of IL-6 and CCL2, upregulated of the reporter CEBPD::SEAP and IL-1ß. To my very best knowledge, this study is the first report of vorinostat-mediated effect on CEBPD and CCL2 gene transcription in THP-1 M $\varphi$.

The simultaneous anti- and pro-inflammatory action of vorinostat is also observed in human monocyte-derived macrophages (HMDMs) ${ }^{268}$ (Table 4.1). A broad dose range of orally administrated vorinostat displays an anti-inflammatory effect in BALB/c mice ${ }^{267}$ (Table 4.1). Studies in HMDMs ${ }^{268}$ and CIA-rat ${ }^{268}$ revealed pro- and anti-inflammatory action of vorinostat depending on its dosage (Table 4.1). In LPS-treated THP-1 cells, low dose vorinostat (0.1 - $0.2 \mathrm{nM}$ ) supresses nuclear accumulation of NF-kB accompanied by the reduced secretion of pro-inflammatory cytokines including IL-6 ${ }^{299}$ (Table 4.1). In this study, vorinostat displayed its simultaneous pro- and anti-inflammatory action only at the early time point, as the IL-1ß gene transcription was not affected 24 hours after M1 treatment. Also in human PBMCs, vorinostat has no effect on LPS-induced IL-1ß mRNA synthesis, 6 and 24 hours after LPS treatment ${ }^{267}$ (Table 4.1).

Considering that HDACs target various transcription-related proteins, ${ }^{123}$ vorinostat may 
control acetylation-sensitive TFs like STAT3 and NF-kB regulating gene transcription

(Fig.4-5). Reversible acetylation of INF-g-responsive STAT3 modulates its transcriptional activity. ${ }^{246,300}$ STAT3 acetylation by CBP/p300 enhances its DNA binding and transactivation, while STAT3 deacetylation by HDAC1-3 inhibits transcription of its target genes. ${ }^{300}$ Vorinostat may induce transcription of IL-1ß and reporter CEBPD::SEAP through the mediation of sustained acetylation of STAT3 and therefore its enhanced activity on target promoters.

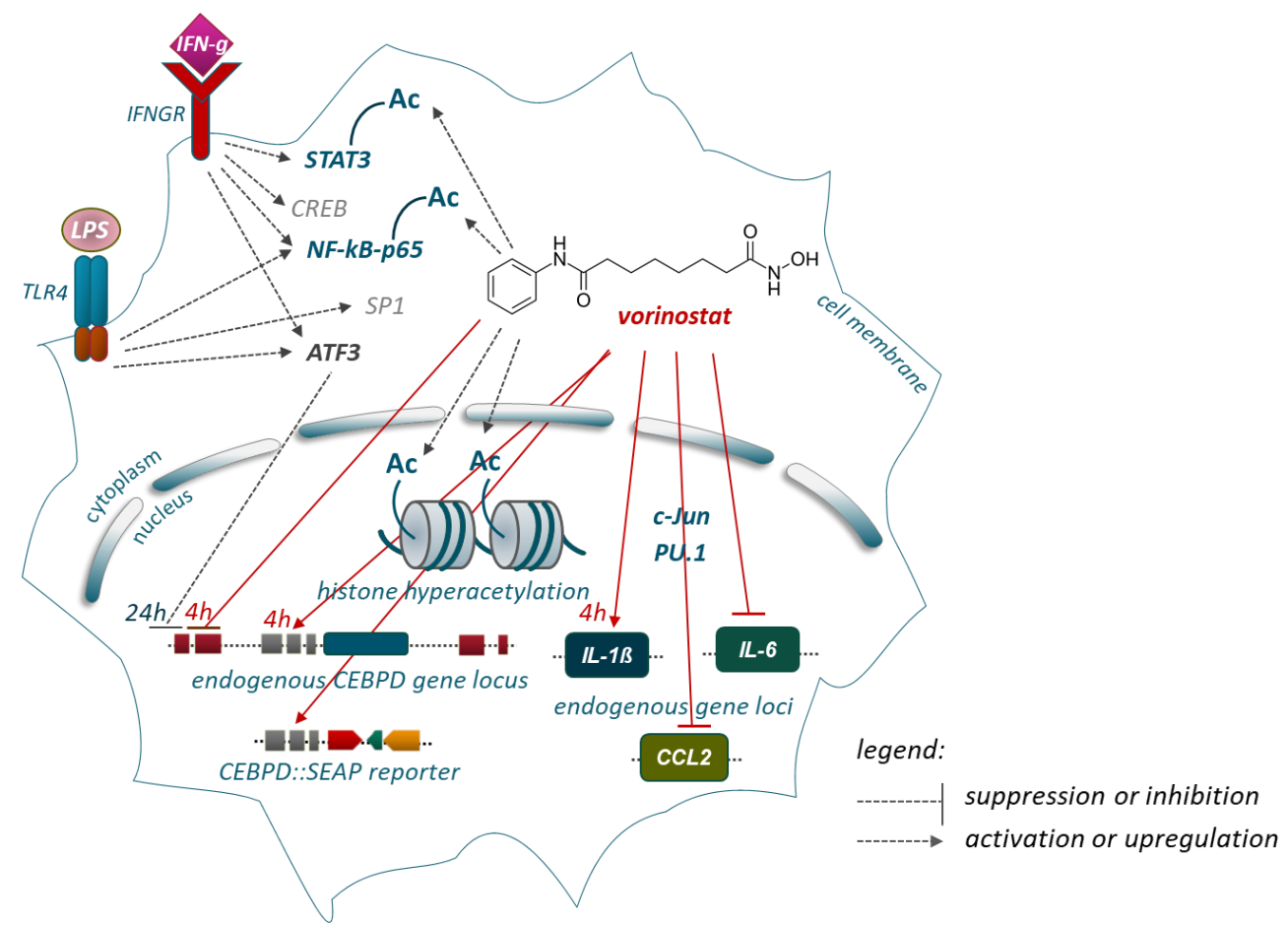

Figure 4-5: The proposed pharmacological activity of HDAC inhibitor vorinostat in M1-polarized THP-1 reporter $\mathrm{M} \varphi$. Vorinostat (red lines) repressed M1 treatment-activated gene expression of IL-6 and CCL2 and upregulated CEBPD::SEAP at both time points tested. However, it completely abolished the M1 treatment-activated gene expression of the endogenous CEBPD and upregulated this of IL-1ß at indicated time points. Vorinostat may control cis-activatory and trans-inhibitory mechanisms simultaneously, the summary action of which results in inhibition of the endogenous CEBPD, but upregulation of the reporter CEBPD::SEAP gene expression, as the defined CEBPD promoter may lack the corresponding regulatory sites. Vorinostat may regulate gene transcription by transcriptional activity control of acetylation-sensitive STAT3 and NF-kB. Thus, vorinostat may cause sustained STAT3 acetylation and control HDAC3-mediated NF-kB nuclear localization resulting in upregulation of its target genes like IL-1ß and the reporter CEBPD::SEAP. Vorinostat may also cause a sustained acetylation of NF-kB Lys122 and Lys123 resulting in downregulation of the endogenous CEBPD, IL-6, and CCL2 gene expression. Vorinostat may also supress CCL2 gene expression by HDAC3 and HDAC11 inhibition, preventing their interaction with SP1, c-Jun and PU.1. For details please refer to the main text.

Acetylation state also regulates the transcriptional activity of NF-kB. ${ }^{301,302}$ Interestingly, sustained NF-kB acetylation, which may be caused by vorinostat, can result in enhanced or reduced NF-kB transcription factor activity. The Lys122 and Lys123 acetylation reduced DNA binding affinity of p65 NF-kB subunit promoting p65-IkB interaction resulting 
in nuclear export of NF-kB. ${ }^{301}$ Consequently, sustained NF-kB Lys122 and Lys123 acetylation in response to vorinostat treatment may reduce transcription of NF-kB target genes like CEBPD and IL-6, while HDAC3 deacetylation of NF-kB subunit RelA causes IkB $\alpha$-dependent nuclear export of NF-kB and therefore supresses target genes' expression. ${ }^{302}$ Thus, vorinostat-mediated HDAC3 inhibition may restore NF-kB nuclear localization and contribute to the enhanced expression of IL-1ß and reporter CEBPD::SEAP genes.

Vorinostat may downregulate CCL2 gene expression through the inhibition of HDAC3 and HDAC11 preventing their interaction with relevant TFs in target cells (Fig.4-5). HDAC3 and HDAC11 are observed to induce CCL2 gene expression in association with different TFs. Interactions of HDAC3 with SP1 and c-Jun ${ }^{303}$ and of HDAC11 with PU.1304 activated CCL2. Vorinostat-mediated reduction in CCL2 gene expression may also result from an altered NF-kB transcriptional activity (as described previously), as CCL2 promoter also contains NF-kB binding site.

\section{Trichostatin A}

HDAC inhibitor TSA had the following effects on the M1 treatment-induced gene expression in THP-1 reporter M $\varphi$ : complete abolishment of the endogenous CEBPD, suppression of IL-6 and CCL2, upregulation of the reporter CEBPD::SEAP and IL-1ß. In contrast to vorinostat, TSA did not affect gene transcription of reporter CEBPD::SEAP, at the late time point. To my best knowledge, this study is the first report of CEBPD expression regulation and IL-1ß gene expression upregulation in THP-1 M $\varphi$.

In murine bone marrow-derived macrophages (mBMDMs), 12.5 and 25 nM TSA doesn't affect IL-1ß mRNA level 6 hours after LPS treatment ${ }^{305}$ (Table 4.1). The observed differences may be caused by the different TSA and LPS concentration used for the cell treatment: $500 \mathrm{nM}$ TSA and $0.1 \mu \mathrm{g} / \mathrm{mL}$ LPS in this study versus up to $25 \mathrm{nM}$ TSA and $1 \mu \mathrm{g} / \mathrm{mL}$ LPS in mBMDMs study. ${ }^{305}$ However, 25 nM TSA slightly decreases IL-1ß secretion from LPS-induced mBMDMs at the later time point ${ }^{305}$ (Table 4.1). The anti-inflammatory effect of TSA is consistent with the literature, as it also reduces pro-inflammatory gene expression and cytokine secretion in $\mathrm{mBMDMs}^{305,306}$ (Table 4.1).

TSA may upregulate gene transcription of the reporter CEBPD::SEAP by acetylation and 
enhancement of SP1 transcriptional activity in target cells (Fig.4-6). TSA increases acetylation of transcription factor SP1 at Lys703 and elevated its DNA binding activity in human Jurkat T cells. ${ }^{307}$ SP1 TF directly binds CEBPD promoter in multiple cell types (cf. chapter 1.3.1.3) and is essential to recruit NF-kB and c-Jun to target promoters in LPSactivated RAW264.7 M.$^{308}$ However, the proposed TSA-dependent SP1 transcriptional

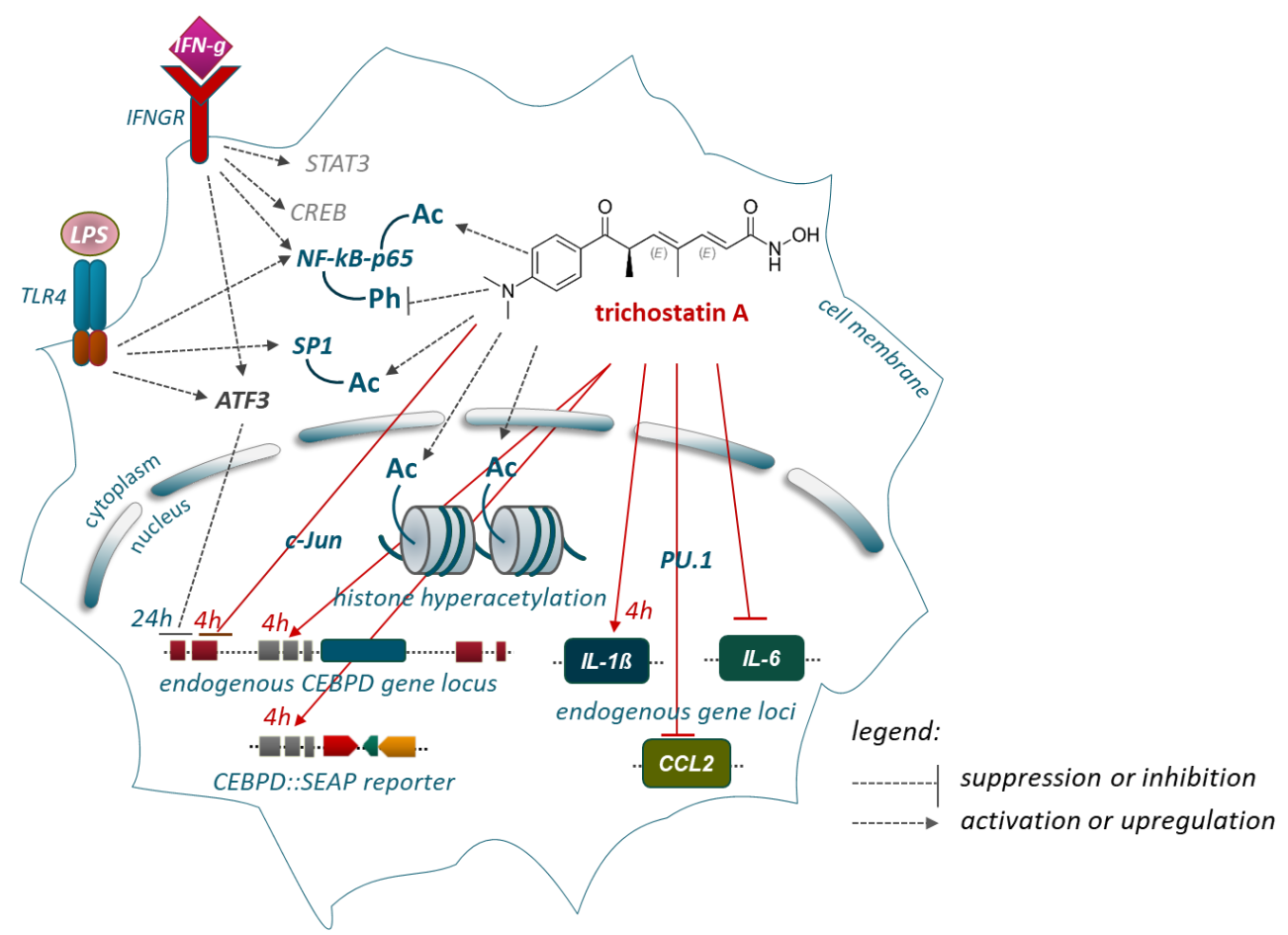

Figure 4-6: The proposed pharmacological activity of HDAC inhibitor trichostatin A in M1-polarized THP1 reporter $\mathrm{M} \varphi$. TSA (red lines) repressed M1 treatment-activated gene expression of IL- 6 and CCL2 at both time points tested. However, it completely abolished the $M 1$ treatment-activated gene expression of the endogenous CEBPD and upregulated that of the reporter CEBPD::SEAP and IL-1ß at the early time point only. TSA may control cis-activatory and trans-inhibitory mechanisms simultaneously, the summary action of which results in inhibition of the endogenous CEBPD but upregulation of the reporter CEBPD::SEAP gene expression, as the defined CEBPD promoter may lack the corresponsive regulatory sites. TSA causes acetylation and thus elevated transcriptional activity of SP1 that may result in upregulated expression of CEBPD::SEAP. On the other hand, TSA may inhibit SP1-dependent c-Jun binding of CEBPD promoter abolishing the activated gene expression of the endogenous CEBPD. TSA may also inhibit expression of the endogenous CEBPD and IL- 6 by: i) preventing of TLR4-dependent NF-kB binding; ii) interruption of IFN-g signalling; iii) regulation of NF-kB acetylation and phosphorylation state. Further, TSA may repress the CCL2 gene expression by mediation of PU.1 transcription factor degradation. For details please refer to the main text.

activity competes with inhibitory mechanisms for endogenous CEBPD gene, as M1 treatment-induced CEBPD gene transcription is abolished in response to TSA treatment. TSAdependent abolishment of LPS-activated transcription of cebpd gene and cebpd mRNA level is also observed in RAW264.7 murine $M \varphi^{309}$ (Table 4-1), by the TSA-mediated inhibition of SP1-dependent c-Jun binding to CEBPD promoter. ${ }^{309}$ 
TSA may supress gene expression of IL- 6 and endogenous CEBPD by preventing TLR4dependent NF-kB-p65 binding and interruption of IFN-g signalling in target cells (Fig.4-6). TSA inhibits production of IFN-g in human U937 cells $\mathrm{s}^{310}$ and is observed to regulate NF-kB-p65 acetylation and phosphorylation balance ${ }^{310}$ affecting the TLR4/ NF-kB-p65 signalling pathway. TSA increases LPS-depressed acetylation and decreased LPS-induced phosphorylation of NF-kB-p65 in U-937 cells. ${ }^{310}$ Pre-treatment with $40 \mathrm{ng} / \mathrm{mL}$ TSA also suppress NF-kB-p65 DNA binding activity and inhibits TLR4 protein expression in LPS-activated RAW264.7, where the acetylation of NF-kB-p65 is increased. ${ }^{311}$

TSA may reduce gene expression of CCL2 through the degradation mediation of a master transcription factor PU.1 (Fig.4-6). PU.1 activates CCL2 expression in $\mathrm{M} \varphi^{306}$ and regulates $\mathrm{M} \varphi$ differentiation at mRNA and protein levels. ${ }^{312}$ TSA supresses expression of PU.1 in multiple murine $\mathrm{M} \varphi$ cell lines including RAW264.7. ${ }^{312}$ Besides transcription regulation of CCL2, an M1 polarization marker, TSA is also observed to facilitate the anti-inflammatory $\mathrm{M} 2$ polarization of $\mathrm{M} \varphi .^{313,314}$

\subsection{Epigenetic action of CEBPD-modulating hit compounds as RA treat- ment strategy}

Environmental-responsive epigenetic factors contribute to the abnormal activation of the innate and adaptive immune systems and also to RA progression. ${ }^{315}$ Flexible acetylation of histones and non-histone proteins regulates production of pro-inflammatory cytokines in synovial $\mathrm{M} \varphi$ and fibroblasts. ${ }^{316}$ The inflammatory stimuli also activate fibroblast-like synoviocytes (FLS) that secrete cytokines and generate enzymes responsible for cartilage and bone destruction. ${ }^{317}$ Cytokine IFN-g ${ }^{193}$ and TLR4 ligand LPS are observed to promote RA by $M \varphi$ activation ${ }^{318}$ and induction of TFs resulting in HAT-mediated increase in histone acetylation and thus transcription initiation of pro-inflammatory genes. $^{319}$

As introduced previously, activated C/EBP $\delta$ TF displays elevated DNA binding activity in synovial $M \varphi$ and fibroblasts derived from RA patient joints ${ }^{164}$ (cf. chapter 1.3.3). Inflam- 
matory-induced C/EBP $\delta$ TF contributes to the transcriptional control of pro-inflammatory cytokine and chemokine-encoding genes in CIA mice. ${ }^{132}$ In the context of RA, identified hit compounds may display their anti-inflammatory effect via their known epigenetically and in this study identified CEBPD-modulating activity.

In general, BETi represent a potential therapeutic strategy for RA, as they display potent anti-inflammatory properties, ${ }^{260}$ accompanied by a good drugability of BCPs. ${ }^{320}$ BET inhibitor GSK-3358699 from Glaxo Smith Kline was in phase I clinical trials for the treatment of RA. ${ }^{8 *}$ Although both GSK $1210151 \mathrm{~A}$ and Ro 11-1464 displayed their anti-inflammatory action in M1-polarized THP-1 reporter $M \varphi$, a potential therapeutic effect in the context of RA is reported only for GSK 1210151A. GSK 1210151A reduces autocrine expression of IFN- $\beta$ and IFN- $\beta$-induced gene transcription resulting in suppressed IFN responses activated by TLR4 and TNF in primary human monocytes. ${ }^{285}$ GSK 1210151A suppresses transcription of over $70 \%$ of genes induced by TNF- $\alpha$ or IL-1ß and reduces secretion of cytokine- and LPS-induced inflammatory mediators such as IL-6 and IL-8 as well as matrix degrading enzymes (MMP1, MMP3), in RA FLSs. ${ }^{261}$ GSK $12010151 \mathrm{~A}$ also reduces proliferation of RA FLSs as well as their chemotactic properties affecting PBMCs. ${ }^{261}$

Ro 11-1464, a potent apoA-I inducer, may restore RA-related disturbance in lipid metabolism and therefore provide beneficial properties in RA treatment. RA patients show reduced serum level of $\mathrm{HDL}$ as well as severe aggregation of apoA-I in $\mathrm{HDL}$ resulting in altered lipoprotein structure and function and correlating with RA-associated cardiovascular events. ${ }^{321}$ In comparison to healthy controls, patients with early RA display significantly lower serum HDL and consequently lower apoA-I levels, which are improved through a DMRAD anti-inflammatory treatment. ${ }^{322}$ Ro 11-1464 may display a double beneficial effect in RA, as it acts not only as apoA-I inducer but also supresses expression of pro-inflammatory genes, as seen in this study.

\footnotetext{
8* Integrity, A Cortellis solution. Clarivate analytics website. Indication: rheumatoid arthritis https://integrity.clarivate.com. Accessed September 24, 2019
} 
HDACi are also observed to display beneficial effects in the context of RA by their versatile anti-inflammatory action. HDAC6 inhibitor CKD-506 from Chong Kun Dang Pharmaceutical was in phase II clinical trial for the treatment of RA. ${ }^{9 *}$ Vorinostat induces apoptosis of primary FLSs from RA patient through the generation of ROS and suppression of NF-kB and anti-apoptotic proteins Bcl-xl and $\mathrm{Mcl}-1 .^{323}$ Vorinostat inhibits cell proliferation and suppresses LPS-induced NF-kB-p65 nuclear accumulation and cytokine secretion in human RA synovial fibroblastic E11 cells, including IL-6. ${ }^{299}$ In E11 cells, vorinostat suppresses monocyte chemotactic protein-2 (MCP-2) and macrophage migration inhibitory factor (MIF) relevant for E11-driven THP-1 migration. ${ }^{269}$ In THP-1 cells, vorinostat supresses LPS-induced NF-kB-p65 nuclear accumulation and secretion of IL-1B, IL-6, IL-18, and TNF- $\alpha .{ }^{299}$

TSA effectively blocks production of IL- 6 and TNF- $\alpha$ and downregulated anti-apoptotic $\mathrm{Bfl}-1 / \mathrm{A} 1$ protein resulting in induced $\mathrm{M} \varphi$ apoptosis in PBMCs derived from healthy donors and RA patients. ${ }^{273}$ TSA suppress IL-1ß-, TNF- $\alpha-$, and TLR ligand-induced IL-6 production through the reduction of IL-6 mRNA stability in FLSs from RA patients and M $\varphi$ from healthy donors. ${ }^{253}$ TSA reduces expression of NF-kB signalling interplayers including $\mathrm{PI} 3 \mathrm{~K}$ and $\mathrm{p}-\mathrm{Akt}^{324}$ as well as inhibited cell viability through cell sensitization toward TNF- $\alpha$-related apoptosis and induction of cell cycle arrest in RA FLSs. ${ }^{325}$

The by LPS and IFN-g activated gene transcription of the endogenous CEBPD was upregulated by BETi and completely inhibited by HDACi in THP-1 reporter M $\varphi$. Pro-inflammatory IL-6 and CCL2 gene transcription was suppressed by all four hit compounds, independently from CEBPD promoter activity. Nonetheless, C/EBP $\delta$ TF may contribute to the suppressed gene expression of IL- 6 and CCL2 in THP-1 reporter $M \varphi$.

Activated C/EBPS TF can act as an anti-inflammatory, as observed in murine $M \varphi,{ }^{130}$ sepsis mice, ${ }^{156}$ and brain inflammation. ${ }^{155}$ The activatory or inhibitory transcriptional action of C/EBPS TF is determined by heterodimerization, post-translational modifications, or interaction with regulatory proteins ( $c f$. chapter 1.3.1.3). Activated C/EBP $\delta$ TF can also repress expression of its target genes, ${ }^{38}$ besides its known action as activator of anti-

\footnotetext{
9* Integrity, A Cortellis solution. Clarivate analytics website. Indication: rheumatoid arthritis https://integrity.clarivate.com. Accessed September 24, 2019
} 
inflammatory genes. ${ }^{130}$ IL-6 and CCL2 promoter regions display C/EBP $\delta$ binding sites, $^{131,132,326}$ however, there is no experimental conformation of the direct CCL2 promoter binding by C/EBP $\delta$ yet. C/EBPS TF upregulated by GSK 1201151A or Ro 11-1464 may display an inhibitory transcriptional activity as a result of heterodimerization with LIP, C/EBPy or CHOP, SUMOylation, or interaction with inhibitory co-regulators like Rad, DIPA, Smad3, or Smad4 (cf. chapter 1.3.1.3) repressing IL-6 and CCL2 expression (Fig.4-7A).

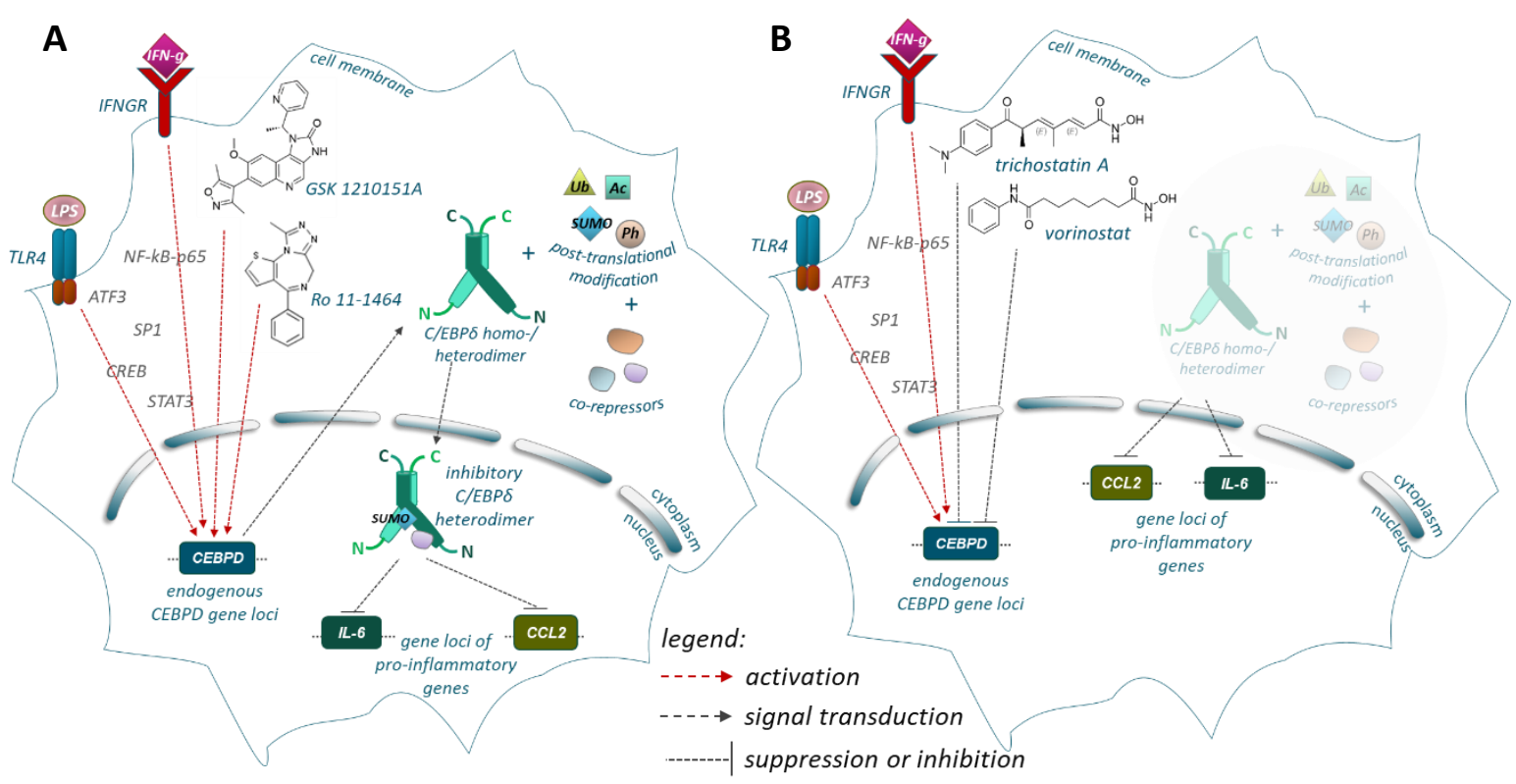

Figure 4-7: The proposed anti-inflammatory activity of CEBPD-modulating hit compounds. Modulation of CEBPD gene expression by identified hit compounds may contribute to their anti-inflammatory activity in M1-polarized THP-1 reporter M $\varphi$. A: BETi GSK 1210151A and Ro 11-1464 upregulated the M1 treatment-activated gene expression of endogenous CEBPD (red arrows). Upregulated C/EBP $\delta$ TF may inhibit IL-6 and CCL2 through its hetero-dimerization with inhibitory mono-dimers, SUMOylation, or interaction with co-repressors. B: HDAC inhibitors vorinostat and TSA completely abolish the M1 treatment-induced expression of endogenous CEBPD (black lines). An absent C/EBP $\delta$ transcriptional activity may contribute to the suppressed expression of IL-6 and CCL2, as observed for cebpd deficiency in murine $M \varphi$.

Absence of C/EBPS TF, due to the inhibited CEBPD gene transcription by vorinostat and TSA, may also contribute to the suppressed IL-6 and CCL2 gene transcription (Fig.4-7B). C/EBP $\delta$-deficiency results in reduced induction of TLR-activated IL-6 gene transcription, as observed in C/EBP $\delta$-null M $\varphi .{ }^{34,150,151} \mathrm{C} / \mathrm{EBP} \delta$-deficiency in mice also reduces systemic inflammation underscored by decreased IL- 6 plasma levels ${ }^{152}$ and attenuated IL- 6 gene expression in brain tissue. ${ }^{153}$ 


\section{Outlook}

This study aimed to develop a flexible, reliable, and sensitive phenotypic screening assay to identify CEBPD-modulating compounds and to analyse the identified hit compounds according to their anti-inflammatory activity in M1-polarized THP-1 reporter $M \varphi$. The results of this study may be expanded into the following research tasks: i) improvement of the screening assay reliability; ii) analysis of hit compound-modulated signalling mediating changes in CEBPD gene expression; iii) analysis of C/EBPס-mediated gene expression control in target cells.

\section{Improvement of the screening assay reliability}

Expression of multi-gene-reporter cassette on a BAC could overcome the observed inconsistences in gene transcription between the reporter and the endogenous CEBPD and thus improve the screening assay reliability. BAC clone $\mathrm{CH} 17-293 \mathrm{~N} 3$ encodes CEBPD and the corresponding regulatory sequences, which can locate in upstream and downstream regions kilobases apart from TSS. In human keratinocytes, for example, p63 binds to a conserved cluster about 1,000 base pairs upstream off the CEBPD transcription start site resulting in decreased C/EBP $\delta$ mRNA levels. ${ }^{327}$ On BAC, the multi-gene-reporter cassette could be expressed, therefore, in the endogenous CEBPD gene environment.

The use of the constructed multi-gene-reporter cassette 2.0 may further improve the screening assay reliability, as it enables ER-stress monitoring. In HEK293T cells, SEAP secretion level was significantly diminished in response to the thapsigargin-induced ER-stress (chapter 3.2.2.1, Fig.3-8). ER-stress-dependent reduction of SEAP secretion, potentially caused by the screening compound treatment, can be misinterpreted as inhibited CEBPD gene transcription. Moreover, ER-stress itself represents a potential object for the study, as it induces an inflammatory response $M \varphi$, fat cells and insulin-producing beta-cells and is, therefore, linked to the pathology of inflammatory disorders. ${ }^{328}$ The preliminary results showed that the multi-gene-reporter cassette 2.0 may enable ER-stress detection by a dual localization mechanism (chapter 3.2.2.1, Fig.3-8). 


\section{Analysis of hit compound-modulated signalling}

From literature, I proposed HDACi and BETi hit compounds having versatile pharmacological effects, which may explain observed changes in CEBPD gene expression (Fig.4-3, Fig.4-4, Fig.4-5, Fig.4-6). ChIP-qPCR analysis of CEBPD promoter can identify regulatory transcription factors directly controlling CEBPD gene expression in THP-1 reporter $M \varphi$. Chromatin immunoprecipitation (ChIP) coupled with quantitative PCR (ChIP-qPCR) is a molecular-biologic method used to investigate protein-DNA interactions at genomic binding sites of the TFs. After regulatory TFs are identified, downstream signalling cascade members can be identified by pull-down assays and Western Blotting.

\section{Analysis of C/EBPS-mediated gene expression control}

C/EBP homo- and heterodimers display minimal differential selectivity for DNA target sites, due to the fully conservation of bZIP module within the bZIP superfamily. ${ }^{34,103} \mathrm{At}$ least in vitro, different C/EBP members bind identical DNA recognition sequences in promoter regions of target genes. ${ }^{56}$ Consequently, target genes of particular C/EBP family member cannot be predicted from the DNA sequence. ${ }^{38}$ The ChIP-qPCR analysis may, therefore, reveal the genes that are regulated by the direct binding of C/EBP $\delta$ to their promoters in M1-polarized THP-1 reporter $\mathrm{M} \varphi$. 


\section{Abbreviations}

\begin{tabular}{|c|c|}
\hline${ }^{\circ} \mathrm{C}$ & degree Celsius \\
\hline$\Delta$ & delta \\
\hline$\mu$ & micro $\left(10^{-6}\right)$ \\
\hline АСТВ & B-actin \\
\hline BAC & bacterial artificial chromosome \\
\hline BETi & bromodomain and extraterminal inhibitor(s) \\
\hline bp & base pairs \\
\hline BSA & bovine serum albumin \\
\hline $\mathrm{CCL}$ & chemokine ( $\mathrm{C}-\mathrm{C}$ motif) \\
\hline cDNA & complementary deoxyribonucleic acid ligand \\
\hline CDS & coding region \\
\hline CEBPD & CCAAT enhancer binding protein delta \\
\hline CV & coefficient of variation \\
\hline $\mathrm{Da}$ & Dalton \\
\hline $\mathrm{ddH}_{2} \mathrm{O}$ & double-distilled water \\
\hline DMSO & dimethyl sulfoxide \\
\hline DNA & deoxyribonucleic acid \\
\hline dNTP & deoxyribonucleotid \\
\hline E. coli & Escherichia coli \\
\hline EDTA & ethylendiamintetraacetate \\
\hline ER & endoplasmic reticulum \\
\hline $\mathrm{F}$ & forward \\
\hline FCS & fetal calf serum \\
\hline g & gram \\
\hline$g$ & gravity of Earth \\
\hline GFP & green fluorescent protein \\
\hline GLuc & Gaussia luciferase \\
\hline GRP78 & glucose-regulated protein 78 \\
\hline h & hour \\
\hline HDACi & histone deacetylase inhibitor(s) \\
\hline HEK & human embryonic kidney \\
\hline HRP & horseradish peroxidase \\
\hline HTS & High-throughput screening \\
\hline IF & Immunofluorescence \\
\hline IFN & interferon \\
\hline IL & interleukin \\
\hline JAK & Janus kinases \\
\hline kb & kilobase pairs \\
\hline L & litre \\
\hline LB & Luria Broth \\
\hline LPS & lipopolysaccharide \\
\hline $\mathrm{m}$ & meter or milli $\left(10^{-3}\right)$ \\
\hline M & molar [mole/litre] \\
\hline mg & milligram \\
\hline $\min$ & minute \\
\hline $\mathrm{mL}$ & millilitre \\
\hline mRNA & messenger ribonucleic acid \\
\hline $\mathrm{MW}$ & molecular weight \\
\hline myc & myc tag \\
\hline
\end{tabular}




\begin{tabular}{|c|c|}
\hline MyD88 & myeloid differentiation primary response 88 \\
\hline $\mathrm{M} \varphi$ & macrophages \\
\hline $\mathrm{n}$ & nano $\left(10^{-9}\right)$ or number of independent experiments \\
\hline NCBI & National Center for Biotechnology Information \\
\hline NF-kB & nuclear factor-kB \\
\hline nt & nucleotide \\
\hline OD & optical density \\
\hline ORF & open reading frame \\
\hline $\mathrm{p}$ & pico $\left(10^{-12}\right)$ \\
\hline PAGE & polyacrylamide gel electrophoresis \\
\hline PBMC & peripheral blood monocyte cell \\
\hline PBS & phosphate buffered saline \\
\hline PCR & polymerase chain reaction \\
\hline PLAP & placental alkaline phosphatase \\
\hline PMA & phorbol 12-myristate, 13-acetate \\
\hline Prl & prolactin \\
\hline Prp & prion protein \\
\hline $\mathrm{P} / \mathrm{S}$ & penicillin/streptomycin \\
\hline PVDF & polyvinylidene fluoride \\
\hline $\mathrm{R}$ & reverse \\
\hline RA & rheumatoid arthritis \\
\hline RNA & ribonucleic acid \\
\hline RPL37A & ribosomal protein L37a \\
\hline rpm & round per minute \\
\hline RT-PCR & reverse transcriptase-polymerase chain reaction \\
\hline SD & standard deviation \\
\hline SDS & sodium dodecyl sulphate \\
\hline SEAP & secreted alkaline phosphatase \\
\hline sec & second \\
\hline SP & signal peptide \\
\hline STAT & signal transducer and activator of transcription \\
\hline TF & transcription factor \\
\hline TLR & toll-like-receptor \\
\hline TNF- $\alpha$ & tumor necrosis factor- $\alpha$ \\
\hline TSA & trichostatin A \\
\hline TSS & transcription start site \\
\hline UTR & untranslated region \\
\hline $\mathrm{v} / \mathrm{v}$ & volume to volume ration \\
\hline$w / v$ & weight to volume ratio \\
\hline WB & Western Blotting \\
\hline wt & wild type \\
\hline$x$ & $x$ times \\
\hline
\end{tabular}

\section{Amino acids}

$\begin{array}{lll}\text { K } & \text { Lys } & \text { lysine } \\ \text { S } & \text { Ser } & \text { serine } \\ \text { T } & \text { Thr } & \text { threonine }\end{array}$




\section{References}

1. Zoellner JM, Porter KJ. Translational Research: Concepts and Methods in Dissemination and Implementation Research. In: Nutrition in the Prevention and Treatment of Disease. Elsevier; 2017:125-143.

2. Swinney DC, Anthony J. How were new medicines discovered? Nature reviews Drug discovery. 2011;10(7):507-519.

3. Eder J, Sedrani R, Wiesmann C. The discovery of first-in-class drugs: origins and evolution. Nat Rev Drug Discov. 2014;13(8):577-587.

4. Vincent $F$, Loria $P$, Pregel $M$, et al. Developing predictive assays: the phenotypic screening "rule of 3". Sci Transl Med. 2015;7(293):293ps215.

5. Zheng W, Thorne N, McKew JC. Phenotypic screens as a renewed approach for drug discovery. Drug Discov Today. 2013;18(21-22):1067-1073.

6. Parnham MJ, Geisslinger G. Pharmacological plasticity-How do you hit a moving target? Pharmacol Res Perspect. 2019;7(6):e00532.

7. Filippakopoulos P, Qi J, Picaud S, et al. Selective inhibition of BET bromodomains. Nature. 2010;468(7327):1067-1073.

8. Sul JY, Wu CW, Zeng $\mathrm{F}$, et al. Transcriptome transfer produces a predictable cellular phenotype. Proc Natl Acad Sci U S A. 2009;106(18):7624-7629.

9. Kain SR, Ganguly S. Overview of genetic reporter systems. Curr Protoc Mol Biol. 2001;Chapter 9:Unit9 6.

10. Anson D. Reporter genes: a practical guide. Vol 411: Springer Science \& Business Media; 2007.

11. Michelini E, Cevenini L, Mezzanotte L, Coppa A, Roda A. Cell-based assays: fuelling drug discovery. Analytical and bioanalytical chemistry. 2010;398(1):227238.

12. Roda A, Pasini P, Mirasoli M, Michelini E, Guardigli M. Biotechnological applications of bioluminescence and chemiluminescence. Trends Biotechnol. 2004;22(6):295-303.

13. Wolff M, Kredel S, Wiedenmann J, Nienhaus GU, Heilker R. Cell-based assays in practice: cell markers from autofluorescent proteins of the GFP-family. Comb Chem High Throughput Screen. 2008;11(8):602-609.

14. Chiba T, Tsuchiya T, Mori R, Shimokawa I. Protein reporter bioassay systems for the phenotypic screening of candidate drugs: a mouse platform for anti-aging drug screening. Sensors (Basel). 2012;12(2):1648-1656.

15. Feng Y, Mitchison TJ, Bender A, Young DW, Tallarico JA. Multi-parameter phenotypic profiling: using cellular effects to characterize small-molecule compounds. Nature Reviews Drug Discovery. 2009;8(7):567-578.

16. Berger J, Hauber J, Hauber R, Geiger R, Cullen BR. Secreted placental alkaline phosphatase: a powerful new quantitative indicator of gene expression in eukaryotic cells. Gene. 1988;66(1):1-10.

17. Li Y, Wang $X$, Ren J, et al. Identification and application of anti-inflammatory compounds screening system based on RAW264. 7 cells stably expressing NF-KBdependent SEAP reporter gene. BMC Pharmacology and Toxicology. 2017;18(1):5. 
18. Yang H, Fung SY, Bao A, Li Q, Turvey SE. Screening bioactive nanoparticles in phagocytic immune cells for inhibitors of toll-like receptor signaling. JoVE (Journal of Visualized Experiments). 2017(125):e56075.

19. Hiramatsu N, Kasai A, Hayakawa K, Yao J, Kitamura M. Real-time detection and continuous monitoring of ER stress in vitro and in vivo by ES-TRAP: evidence for systemic, transient ER stress during endotoxemia. Nucleic Acids Res. 2006;34(13):e93.

20. Bettan M, Darteil R, Scherman D. Secreted human placental alkaline phosphatase as a reporter gene for in vivo gene transfer. Analytical biochemistry. 1999;271(2):187-189.

21. Schlatter S, Rimann M, Kelm J, Fussenegger M. SAMY, a novel mammalian reporter gene derived from Bacillus stearothermophilus alpha-amylase. Gene. 2002;282(1-2):19-31.

22. Cullen BR, Malim MH. Secreted placental alkaline phosphatase as a eukaryotic reporter gene. Methods Enzymol. 1992;216:362-368.

23. Szent-Gyorgyi C, Ballou BT, Dagnal E, Bryan B. Cloning and characterization of new bioluminescent proteins. Paper presented at: Biomedical Imaging: Reporters, Dyes, and Instrumentation1999.

24. Bryan BJ, Szent-Gyorgyi C. Luciferases, fluorescent proteins, nucleic acids encoding the luciferases and fluorescent proteins and the use thereof in diagnostics, high throughput screening and novelty items. In: Google Patents; 2001.

25. Wu N, Rathnayaka T, Kuroda Y. Bacterial expression and re-engineering of Gaussia princeps luciferase and its use as a reporter protein. Biochimica et Biophysica Acta (BBA)-Proteins and Proteomics. 2015;1854(10):1392-1399.

26. Ruecker O, Zillner K, Groebner-Ferreira R, Heitzer M. Gaussia-luciferase as a sensitive reporter gene for monitoring promoter activity in the nucleus of the green alga Chlamydomonas reinhardtii. Mol Genet Genomics. 2008;280(2):153162.

27. Badr CE, Hewett JW, Breakefield XO, Tannous BA. A highly sensitive assay for monitoring the secretory pathway and ER stress. PLoS One. 2007;2(6):e571.

28. Remy I, Michnick SW. A highly sensitive protein-protein interaction assay based on Gaussia luciferase. Nat Methods. 2006;3(12):977-979.

29. Tannous BA, Kim DE, Fernandez JL, Weissleder R, Breakefield XO. Codonoptimized Gaussia luciferase cDNA for mammalian gene expression in culture and in vivo. Mol Ther. 2005;11(3):435-443.

30. Inouye S, Sahara-Miura Y, Sato J-i, limori R, Yoshida S, Hosoya T. Expression, purification and luminescence properties of coelenterazine-utilizing luciferases from Renilla, Oplophorus and Gaussia: comparison of substrate specificity for C2modified coelenterazines. Protein Expression and Purification. 2013;88(1):150156.

31. Wurdinger $T$, Badr C, Pike $L$, et al. A secreted luciferase for ex vivo monitoring of in vivo processes. Nat Methods. 2008;5(2):171-173.

32. Ramji DP, Foka P. CCAAT/enhancer-binding proteins: structure, function and regulation. Biochem J. 2002;365(Pt 3):561-575.

33. Landschulz WH, Johnson PF, Adashi EY, Graves BJ, McKnight SL. Isolation of a recombinant copy of the gene encoding C/EBP. Genes Dev. 1988;2(7):786-800. 
34. Tsukada J, Yoshida Y, Kominato Y, Auron PE. The CCAAT/enhancer (C/EBP) family of basic-leucine zipper (bZIP) transcription factors is a multifaceted highlyregulated system for gene regulation. Cytokine. 2011;54(1):6-19.

35. Cao Z, Umek RM, McKnight SL. Regulated expression of three C/EBP isoforms during adipose conversion of 3T3-L1 cells. Genes Dev. 1991;5(9):1538-1552.

36. Pulido-Salgado $M$, Vidal-Taboada JM, Saura J. C/EBP $\beta$ and C/EBP $\delta$ transcription factors: basic biology and roles in the CNS. Progress in neurobiology. 2015;132:133.

37. Dearth LR, DeWille J. Posttranscriptional and posttranslational regulation of C/EBP delta in GO growth-arrested mammary epithelial cells. J Biol Chem. 2003;278(13):11246-11255.

38. Balamurugan $K$, Sterneck E. The many faces of C/EBPdelta and their relevance for inflammation and cancer. Int J Biol Sci. 2013;9(9):917-933.

39. Hurst HC. Transcription factors 1: bZIP proteins. Protein Profile. 1995;2(2):101168.

40. Leutz A, Pless O, Lappe M, Dittmar G, Kowenz-Leutz E. Crosstalk between phosphorylation and multi-site arginine/lysine methylation in C/EBPs. Transcription. 2011;2(1):3-8.

41. Landschulz WH, Johnson PF, McKnight SL. The DNA binding domain of the rat liver nuclear protein C/EBP is bipartite. Science. 1989;243(4899):1681-1688.

42. Williams SC, Angerer ND, Johnson PF. C/EBP proteins contain nuclear localization signals imbedded in their basic regions. Gene Expr. 1997;6(6):371-385.

43. Landschulz WH, Johnson PF, McKnight SL. The leucine zipper: a hypothetical structure common to a new class of DNA binding proteins. Science. 1988;240(4860):1759-1764.

44. Petosa C, Morand P, Baudin F, Moulin M, Artero JB, Muller CW. Structural basis of lytic cycle activation by the Epstein-Barr virus ZEBRA protein. Mol Cell. 2006;21(4):565-572.

45. Listman JA, Wara-aswapati N, Race JE, et al. Conserved ETS domain arginines mediate DNA binding, nuclear localization, and a novel mode of bZIP interaction. J Biol Chem. 2005;280(50):41421-41428.

46. Williamson EA, $\mathrm{Xu} \mathrm{HN}, \mathrm{Gombart} A \mathrm{~F}$, et al. Identification of transcriptional activation and repression domains in human CCAAT/enhancer-binding protein epsilon. J Biol Chem. 1998;273(24):14796-14804.

47. Dyson HJ, Wright PE. Intrinsically unstructured proteins and their functions. Nat Rev Mol Cell Biol. 2005;6(3):197-208.

48. Kowenz-Leutz E, Pless O, Dittmar G, Knoblich M, Leutz A. Crosstalk between C/EBPbeta phosphorylation, arginine methylation, and SWI/SNF/Mediator implies an indexing transcription factor code. EMBO J. 2010;29(6):1105-1115.

49. Kowenz-Leutz E, Leutz A. A C/EBP beta isoform recruits the SWI/SNF complex to activate myeloid genes. Mol Cell. 1999;4(5):735-743.

50. Mo X, Kowenz-Leutz E, Xu H, Leutz A. Ras induces mediator complex exchange on C/EBP beta. Mol Cell. 2004;13(2):241-250.

51. Mink S, Haenig B, Klempnauer KH. Interaction and functional collaboration of p300 and C/EBPbeta. Mol Cell Biol. 1997;17(11):6609-6617. 
52. Kim J, Cantwell CA, Johnson PF, Pfarr CM, Williams SC. Transcriptional activity of CCAAT/enhancer-binding proteins is controlled by a conserved inhibitory domain that is a target for sumoylation. J Biol Chem. 2002;277(41):38037-38044.

53. Balamurugan $\mathrm{K}$, Wang JM, Tsai $\mathrm{HH}$, et al. The tumour suppressor C/EBPdelta inhibits FBXW7 expression and promotes mammary tumour metastasis. EMBO J. 2010;29(24):4106-4117.

54. Billiard J, Umayahara Y, Wiren K, Centrella M, McCarthy TL, Rotwein P. Regulated nuclear-cytoplasmic localization of CCAAT/enhancer-binding protein delta in osteoblasts. J Biol Chem. 2001;276(18):15354-15361.

55. Wang J, Sarkar TR, Zhou $M$, et al. CCAAT/enhancer binding protein delta (C/EBPdelta, CEBPD)-mediated nuclear import of FANCD2 by IPO4 augments cellular response to DNA damage. Proc Natl Acad Sci U S A. 2010;107(37):1613116136.

56. Lekstrom-Himes J, Xanthopoulos KG. Biological role of the CCAAT/enhancerbinding protein family of transcription factors. J Biol Chem. 1998;273(44):2854528548.

57. Wedel A, Ziegler-Heitbrock HW. The C/EBP family of transcription factors. Immunobiology. 1995;193(2-4):171-185.

58. Tengku-Muhammad TS, Hughes TR, Ranki H, Cryer A, Ramji DP. Differential regulation of macrophage CCAAT-enhancer binding protein isoforms by lipopolysaccharide and cytokines. Cytokine. 2000;12(9):1430-1436.

59. Rouillard AD, Gundersen GW, Fernandez NF, et al. The harmonizome: a collection of processed datasets gathered to serve and mine knowledge about genes and proteins. Database (Oxford). 2016;2016.

60. Hallenborg P, Feddersen S, Francoz S, et al. Mdm2 controls CREB-dependent transactivation and initiation of adipocyte differentiation. Cell Death Differ. 2012;19(8):1381-1389.

61. Huang YC, Chang WC, Su JG, et al. Peptidoglycan enhances transcriptional expression of CCAAT/enhancer-binding protein delta gene in mouse macrophages. J Biomed Sci. 2007;14(3):407-418.

62. Liu Y-W, Chen C-C, Wang J-M, et al. Role of transcriptional factors Sp1, c-Rel, and c-Jun in LPS-induced C/EBP $\delta$ gene expression of mouse macrophages. Cellular and molecular life sciences. 2007;64(24):3282-3294.

63. Gong B, Pan Y, Zhao W, et al. IVIG immunotherapy protects against synaptic dysfunction in Alzheimer's disease through complement anaphylatoxin C5amediated AMPA-CREB-C/EBP signaling pathway. Mol Immunol. 2013;56(4):619629.

64. Cantwell CA, Sterneck E, Johnson PF. Interleukin-6-specific activation of the C/EBPdelta gene in hepatocytes is mediated by Stat3 and Sp1. Mol Cell Biol. 1998;18(4):2108-2117.

65. Sivko GS, Sanford DC, Dearth LD, Tang D, DeWille JW. CCAAT/Enhancer binding protein delta (c/EBPdelta) regulation and expression in human mammary epithelial cells: II. Analysis of activating signal transduction pathways, transcriptional, post-transcriptional, and post-translational control. J Cell Biochem. 2004;93(4):844-856.

66. Ceccarelli V, Racanicchi S, Martelli MP, et al. Eicosapentaenoic acid demethylates a single CpG that mediates expression of tumor suppressor CCAAT/enhancer- 
binding protein $\delta$ in U937 leukemia cells. Journal of Biological Chemistry. 2011;286(31):27092-27102.

67. Tang D, Sivko G, DeWille J. Promoter methylation reduces C/EBPS (CEBPD) gene expression in the SUM-52PE human breast cancer cell line and in primary breast tumors. Breast cancer research and treatment. 2006;95(2):161-170.

68. Yamada T, Tobita K, Osada S, Nishihara T, Imagawa M. CCAAT/enhancer-binding protein delta gene expression is mediated by APRF/STAT3. I Biochem. 1997;121(4):731-738.

69. Zhang Y, Sif S, DeWille J. The mouse C/EBPdelta gene promoter is regulated by STAT3 and Sp1 transcriptional activators, chromatin remodeling and c-Myc repression. J Cell Biochem. 2007;102(5):1256-1270.

70. Ali S, Singh NN, Yildirim H, Ramji DP. Requirement for nuclear factor kappa B signalling in the interleukin-1-induced expression of the CCAAT/enhancer binding protein-delta gene in hepatocytes. Int J Biochem Cell Biol. 2010;42(1):113-119.

71. Litvak V, Ramsey SA, Rust AG, et al. Function of C/EBPdelta in a regulatory circuit that discriminates between transient and persistent TLR4-induced signals. Nat Immunol. 2009;10(4):437-443.

72. Palmieri $C$, Monteverde M, Lattanzio L, et al. Site-specific CpG methylation in the CCAAT/enhancer binding protein delta (CEBPdelta) CpG island in breast cancer is associated with metastatic relapse. Br J Cancer. 2012;107(4):732-738.

73. Li B, Si J, DeWille JW. Ultraviolet radiation (UVR) activates p38 MAP kinase and induces post-transcriptional stabilization of the C/EBPdelta mRNA in G0 growth arrested mammary epithelial cells. J Cell Biochem. 2008;103(5):1657-1669.

74. Hsiao YW, Li CF, Chi JY, et al. CCAAT/enhancer binding protein delta in macrophages contributes to immunosuppression and inhibits phagocytosis in nasopharyngeal carcinoma. Sci Signal. 2013;6(284):ra59.

75. Banerjee $\mathrm{S}, \mathrm{Xie} \mathrm{N}$, Cui $\mathrm{H}$, et al. MicroRNA let-7c regulates macrophage polarization. J Immunol. 2013;190(12):6542-6549.

76. Nerlov C. C/EBPs: recipients of extracellular signals through proteome modulation. Curr Opin Cell Biol. 2008;20(2):180-185.

77. Ray A, Ray BK. Serum amyloid A gene expression under acute-phase conditions involves participation of inducible C/EBP-beta and C/EBP-delta and their activation by phosphorylation. Mol Cell Biol. 1994;14(6):4324-4332.

78. Ko CY, Wang WL, Wang SM, Chu YY, Chang WC, Wang JM. Glycogen synthase kinase-3beta-mediated CCAAT/enhancer-binding protein delta phosphorylation in astrocytes promotes migration and activation of microglia/macrophages. Neurobiol Aging. 2014;35(1):24-34.

79. Kravchenko VV, Mathison JC, Schwamborn K, Mercurio F, Ulevitch RJ. IKKi/IKKepsilon plays a key role in integrating signals induced by proinflammatory stimuli. J Biol Chem. 2003;278(29):26612-26619.

80. Schwind L, Zimmer AD, Gotz C, Montenarh M. CK2 phosphorylation of C/EBPdelta regulates its transcription factor activity. Int J Biochem Cell Biol. 2015;61:81-89.

81. Chamberlain W, Gonnella P, Alamdari N, Aversa Z, Hasselgren PO. Multiple muscle wasting-related transcription factors are acetylated in dexamethasonetreated muscle cells. Biochem Cell Biol. 2012;90(2):200-208. 
82. Kovács KA, Steinmann $M$, Magistretti PJ, Halfon $O$, Cardinaux J-R. CCAAT/enhancer-binding protein family members recruit the coactivator CREBbinding protein and trigger its phosphorylation. Journal of Biological Chemistry. 2003;278(38):36959-36965.

83. Wang JM, Ko CY, Chen LC, Wang WL, Chang WC. Functional role of NF-IL6beta and its sumoylation and acetylation modifications in promoter activation of cyclooxygenase 2 gene. Nucleic Acids Res. 2006;34(1):217-231.

84. Sarkar TR, Sharan S, Wang J, et al. Identification of a Src tyrosine kinase/SIAH2 E3 ubiquitin ligase pathway that regulates C/EBPdelta expression and contributes to transformation of breast tumor cells. Mol Cell Biol. 2012;32(2):320-332.

85. Zhou S, Si J, Liu T, DeWille JW. PIASy represses CCAAT/enhancer-binding protein delta (C/EBPdelta) transcriptional activity by sequestering C/EBPdelta to the nuclear periphery. J Biol Chem. 2008;283(29):20137-20148.

86. Wang WL, Lee YC, Yang WM, Chang WC, Wang JM. Sumoylation of LAP1 is involved in the HDAC4-mediated repression of COX-2 transcription. Nucleic Acids Res. 2008;36(19):6066-6079.

87. Balamurugan $K$, Sharan $S$, Klarmann KD, et al. FBXW7alpha attenuates inflammatory signalling by downregulating C/EBPdelta and its target gene TIr4. Nat Commun. 2013;4:1662.

88. Grigoryan G, Reinke AW, Keating AE. Design of protein-interaction specificity gives selective bZIP-binding peptides. Nature. 2009;458(7240):859-864.

89. Descombes $P$, Schibler U. A liver-enriched transcriptional activator protein, LAP, and a transcriptional inhibitory protein, LIP, are translated from the same mRNA. Cell. 1991;67(3):569-579.

90. Parkin SE, Baer M, Copeland TD, Schwartz RC, Johnson PF. Regulation of CCAAT/enhancer-binding protein (C/EBP) activator proteins by heterodimerization with C/EBPgamma (Ig/EBP). J Biol Chem. 2002;277(26):23563-23572.

91. Ron D, Habener JF. CHOP, a novel developmentally regulated nuclear protein that dimerizes with transcription factors C/EBP and LAP and functions as a dominant-negative inhibitor of gene transcription. Genes Dev. 1992;6(3):439453.

92. Wingender E, Schoeps T, Haubrock M, Donitz J. TFClass: a classification of human transcription factors and their rodent orthologs. Nucleic Acids Res. 2015;43(Database issue):D97-102.

93. Rouschop KM, van den Beucken $T$, Dubois $L$, et al. The unfolded protein response protects human tumor cells during hypoxia through regulation of the autophagy genes MAP1LC3B and ATG5. J Clin Invest. 2010;120(1):127-141.

94. Chiang BT, Liu YW, Chen BK, Wang JM, Chang WC. Direct interaction of C/EBPdelta and Sp1 at the GC-enriched promoter region synergizes the IL-10 gene transcription in mouse macrophage. J Biomed Sci. 2006;13(5):621-635.

95. Meruvu S, Hugendubler L, Mueller E. Regulation of adipocyte differentiation by the zinc finger protein ZNF638. J Biol Chem. 2011;286(30):26516-26523.

96. Zhang J, Chang L, Chen C, et al. Rad GTPase inhibits cardiac fibrosis through connective tissue growth factor. Cardiovasc Res. 2011;91(1):90-98. 
97. Bezy O, Elabd C, Cochet O, et al. Delta-interacting protein A, a new inhibitory partner of CCAAT/enhancer-binding protein beta, implicated in adipocyte differentiation. J Biol Chem. 2005;280(12):11432-11438.

98. Lai PH, Wang WL, Ko CY, et al. HDAC1/HDAC3 modulates PPARG2 transcription through the sumoylated CEBPD in hepatic lipogenesis. Biochim Biophys Acta. 2008;1783(10):1803-1814.

99. $\mathrm{Xu} \mathrm{L}$, Panel V, Ma X, et al. The winged helix transcription factor Foxa3 regulates adipocyte differentiation and depot-selective fat tissue expansion. Molecular and cellular biology. 2013;33(17):3392-3399.

100. Choy L, Derynck R. Transforming growth factor-beta inhibits adipocyte differentiation by Smad3 interacting with CCAAT/enhancer-binding protein (C/EBP) and repressing C/EBP transactivation function. I Biol Chem. 2003;278(11):9609-9619.

101. Vinson CR, Sigler PB, McKnight SL. Scissors-grip model for DNA recognition by a family of leucine zipper proteins. Science. 1989;246(4932):911-916.

102. Johnson PF. Identification of C/EBP basic region residues involved in DNA sequence recognition and half-site spacing preference. Mol Cell Biol. 1993;13(11):6919-6930.

103. Osada S, Yamamoto H, Nishihara T, Imagawa M. DNA binding specificity of the CCAAT/enhancer-binding protein transcription factor family. I Biol Chem. 1996;271(7):3891-3896.

104. Mahony S, Auron PE, Benos PV. DNA familial binding profiles made easy: comparison of various motif alignment and clustering strategies. PLoS Comput Biol. 2007;3(3):e61.

105. Siersbaek R, Nielsen R, John $\mathrm{S}$, et al. Extensive chromatin remodelling and establishment of transcription factor 'hotspots' during early adipogenesis. EMBO J. 2011;30(8):1459-1472.

106. Gery S, Tanosaki S, Hofmann WK, Koppel A, Koeffler HP. C/EBPdelta expression in a BCR-ABL-positive cell line induces growth arrest and myeloid differentiation. Oncogene. 2005;24(9):1589-1597.

107. Egeblad M, Nakasone ES, Werb Z. Tumors as organs: complex tissues that interface with the entire organism. Dev Cell. 2010;18(6):884-901.

108. Liu P, Cao W, Ma B, et al. Action and clinical significance of CCAAT/enhancerbinding protein delta in hepatocellular carcinoma. Carcinogenesis. 2019;40(1):155-163.

109. Yu Y-j, Xu Y-y, Lan X-o, et al. Shikonin induces apoptosis and suppresses growth in keratinocytes via CEBP- $\delta$ upregulation. International immunopharmacology. 2019;72:511-521.

110. Thangaraju $\mathrm{M}$, Rudelius $\mathrm{M}$, Bierie $\mathrm{B}$, et al. C/EBPdelta is a crucial regulator of proapoptotic gene expression during mammary gland involution. Development. 2005;132(21):4675-4685.

111. Cardinaux JR, Magistretti PJ. Vasoactive intestinal peptide, pituitary adenylate cyclase-activating peptide, and noradrenaline induce the transcription factors CCAAT/enhancer binding protein (C/EBP)-beta and C/EBP delta in mouse cortical astrocytes: involvement in cAMP-regulated glycogen metabolism. J Neurosci. 1996;16(3):919-929. 
112. Taubenfeld SM, Wiig KA, Monti B, Dolan B, Pollonini G, Alberini CM. Fornixdependent induction of hippocampal CCAAT enhancer-binding protein [beta] and [delta] Co-localizes with phosphorylated CAMP response element-binding protein and accompanies long-term memory consolidation. I Neurosci. 2001;21(1):84-91.

113. MacDougald OA, Cornelius P, Lin FT, Chen SS, Lane MD. Glucocorticoids reciprocally regulate expression of the CCAAT/enhancer-binding protein alpha and delta genes in 3T3-L1 adipocytes and white adipose tissue. $J$ Biol Chem. 1994;269(29):19041-19047.

114. MacDougald OA, Cornelius P, Liu R, Lane MD. Insulin regulates transcription of the CCAAT/enhancer binding protein (C/EBP) alpha, beta, and delta genes in fully-differentiated 3T3-L1 adipocytes. J Biol Chem. 1995;270(2):647-654.

115. Wang JM, Tseng JT, Chang WC. Induction of human NF-IL6beta by epidermal growth factor is mediated through the p38 signaling pathway and cAMP response element-binding protein activation in A431 cells. Mol Biol Cell. 2005;16(7):3365-3376.

116. Nau GJ, Richmond JF, Schlesinger A, Jennings EG, Lander ES, Young RA. Human macrophage activation programs induced by bacterial pathogens. Proc Natl Acad Sci U S A. 2002;99(3):1503-1508.

117. Boldrick JC, Alizadeh AA, Diehn $M$, et al. Stereotyped and specific gene expression programs in human innate immune responses to bacteria. Proc Natl Acad Sci U S A. 2002;99(2):972-977.

118. Medzhitov R, Horng T. Transcriptional control of the inflammatory response. Nat Rev Immunol. 2009;9(10):692-703.

119. Ko CY, Chang WC, Wang JM. Biological roles of CCAAT/Enhancer-binding protein delta during inflammation. J Biomed Sci. 2015;22:6.

120. Kinoshita S, Akira S, Kishimoto T. A member of the C/EBP family, NF-IL6 beta, forms a heterodimer and transcriptionally synergizes with NF-IL6. Proceedings of the National Academy of Sciences. 1992;89(4):1473-1476.

121. Alam T, An MR, Papaconstantinou J. Differential expression of three C/EBP isoforms in multiple tissues during the acute phase response. $J$ Biol Chem. 1992;267(8):5021-5024.

122. Cardinaux JR, Allaman I, Magistretti PJ. Pro-inflammatory cytokines induce the transcription factors C/EBPbeta and C/EBPdelta in astrocytes. Glia. 2000;29(1):91-97.

123. Granger RL, Hughes TR, Ramji DP. Stimulus- and cell-type-specific regulation of CCAAT-enhancer binding protein isoforms in glomerular mesangial cells by lipopolysaccharide and cytokines. Biochim Biophys Acta. 2000;1501(2-3):171179.

124. Yin M, Yang SQ, Lin HZ, Lane MD, Chatterjee S, Diehl AM. Tumor necrosis factor alpha promotes nuclear localization of cytokine-inducible CCAAT/enhancer binding protein isoforms in hepatocytes. J Biol Chem. 1996;271(30):1797417978.

125. Sanford DC, DeWille JW. C/EBPdelta is a downstream mediator of IL-6 induced growth inhibition of prostate cancer cells. Prostate. 2005;63(2):143-154. 
126. Ko CY, Chang LH, Lee YC, et al. CCAAT/enhancer binding protein delta (CEBPD) elevating PTX3 expression inhibits macrophage-mediated phagocytosis of dying neuron cells. Neurobiol Aging. 2012;33(2):422 e411-425.

127. Yang $\mathrm{H}$, Mammen J, Wei W, et al. Expression and activity of C/EBPbeta and delta are upregulated by dexamethasone in skeletal muscle. J Cell Physiol. 2005;204(1):219-226.

128. Ji C, Chang W, Centrella M, McCarthy TL. Activation domains of CCAAT enhancer binding protein delta: regions required for native activity and prostaglandin E2dependent transactivation of insulin-like growth factor I gene expression in rat osteoblasts. Mol Endocrinol. 2003;17(9):1834-1843.

129. Sekine O, Nishio Y, Egawa K, Nakamura T, Maegawa H, Kashiwagi A. Insulin activates CCAAT/enhancer binding proteins and proinflammatory gene expression through the phosphatidylinositol 3-kinase pathway in vascular smooth muscle cells. J Biol Chem. 2002;277(39):36631-36639.

130. Liu Y-W, Chen C-C, Tseng H-P, Chang W-C. Lipopolysaccharide-induced transcriptional activation of interleukin-10 is mediated by MAPK-and NF-KBinduced CCAAT/enhancer-binding protein $\delta$ in mouse macrophages. Cellular signalling. 2006;18(9):1492-1500.

131. Poli V. The role of C/EBP isoforms in the control of inflammatory and native immunity functions. J Biol Chem. 1998;273(45):29279-29282.

132. Chang LH, Huang HS, Wu PT, et al. Role of macrophage CCAAT/enhancer binding protein delta in the pathogenesis of rheumatoid arthritis in collagen-induced arthritic mice. PLoS One. 2012;7(9):e45378.

133. Sato $Y$, Nishio $Y$, Sekine $O$, et al. Increased expression of CCAAT/enhancer binding protein-beta and -delta and monocyte chemoattractant protein-1 genes in aortas from hyperinsulinaemic rats. Diabetologia. 2007;50(2):481-489.

134. Maitra U, Gan L, Chang S, Li L. Low-dose endotoxin induces inflammation by selectively removing nuclear receptors and activating CCAAT/enhancer-binding protein $\delta$. The Journal of Immunology. 2011;186(7):4467-4473.

135. Ramji DP, Vitelli A, Tronche F, Cortese R, Ciliberto G. The two C/EBP isoforms, IL6DBP/NF-IL6 and C/EBP delta/NF-IL6 beta, are induced by IL- 6 to promote acute phase gene transcription via different mechanisms. Nucleic Acids Res. 1993;21(2):289-294.

136. Lu X. Impact of Macrophages in Atherosclerosis. Curr Med Chem. 2016;23(18):1926-1937.

137. Ostuni R, Kratochvill F, Murray PJ, Natoli G. Macrophages and cancer: from mechanisms to therapeutic implications. Trends Immunol. 2015;36(4):229-239.

138. Laria A, Lurati A, Marrazza M, Mazzocchi D, Re KA, Scarpellini M. The macrophages in rheumatic diseases. J Inflamm Res. 2016;9:1-11.

139. Kennedy A, Fearon U, Veale DJ, Godson C. Macrophages in synovial inflammation. Front Immunol. 2011;2:52.

140. Kawai T, Akira S. Toll-like receptors and their crosstalk with other innate receptors in infection and immunity. Immunity. 2011;34(5):637-650.

141. Kawai T, Akira S. Pathogen recognition with Toll-like receptors. Curr Opin Immunol. 2005;17(4):338-344.

142. Akira $S$, Uematsu $S$, Takeuchi O. Pathogen recognition and innate immunity. Cell. 2006;124(4):783-801. 
143. Takeda K, Akira S. TLR signaling pathways. Semin Immunol. 2004;16(1):3-9.

144. Jenner RG, Young RA. Insights into host responses against pathogens from transcriptional profiling. Nat Rev Microbiol. 2005;3(4):281-294.

145. Beutler B. TIr4: central component of the sole mammalian LPS sensor. Curr Opin Immunol. 2000;12(1):20-26.

146. Ramsey SA, Klemm SL, Zak DE, et al. Uncovering a macrophage transcriptional program by integrating evidence from motif scanning and expression dynamics. PLoS Comput Biol. 2008;4(3):e1000021.

147. Gordon S. Alternative activation of macrophages. Nat Rev Immunol. 2003;3(1):23-35.

148. Taylor PR, Martinez-Pomares L, Stacey M, Lin HH, Brown GD, Gordon S. Macrophage receptors and immune recognition. Annu Rev Immunol. 2005;23:901-944.

149. Glaros T, Fu Y, Xing J, Li L. Molecular mechanism underlying persistent induction of LCN2 by lipopolysaccharide in kidney fibroblasts. PLoS One. 2012;7(4):e34633.

150. Lu YC, Kim I, Lye E, et al. Differential role for C-Rel and C/EBPbeta/delta in TLRmediated induction of proinflammatory cytokines. I Immunol. 2009;182(11):7212-7221.

151. Yan C, Johnson PF, Tang H, Ye Y, Wu M, Gao H. CCAAT/enhancer-binding protein delta is a critical mediator of lipopolysaccharide-induced acute lung injury. Am J Pathol. 2013;182(2):420-430.

152. Slofstra SH, Groot AP, Obdeijn MH, Reitsma PH, ten Cate H, Spek CA. Gene expression profiling identifies C/EBPdelta as a candidate regulator of endotoxininduced disseminated intravascular coagulation. Am J Respir Crit Care Med. 2007;176(6):602-609.

153. Valente $T$, Straccia $M$, Gresa-Arribas $N$, et al. CCAAT/enhancer binding protein delta regulates glial proinflammatory gene expression. Neurobiol Aging. 2013;34(9):2110-2124.

154. Moore F, Santin I, Nogueira TC, et al. The transcription factor C/EBP delta has anti-apoptotic and anti-inflammatory roles in pancreatic beta cells. PLoS One. 2012;7(2):e31062.

155. Rustenhoven J, Scotter EL, Jansson D, et al. An anti-inflammatory role for C/EBPdelta in human brain pericytes. Sci Rep. 2015;5:12132.

156. Banerjee $\mathrm{S}, \mathrm{Fu} \mathrm{Q}$, Shah SK, et al. C/EBPdelta protects from radiation-induced intestinal injury and sepsis by suppression of inflammatory and nitrosative stress. Sci Rep. 2019;9(1):13953.

157. Takata Y, Kitami Y, Yang ZH, Nakamura M, Okura T, Hiwada K. Vascular inflammation is negatively autoregulated by interaction between CCAAT/enhancer-binding protein-delta and peroxisome proliferator-activated receptor-gamma. Circ Res. 2002;91(5):427-433.

158. Lai H-Y, Hsu L-W, Tsai H-H, et al. CCAAT/enhancer-binding protein delta promotes intracellular lipid accumulation in M1 macrophages of vascular lesions. Cardiovascular Research. 2017;113(11):1376-1388.

159. Gao H, Bryzgalova G, Hedman E, et al. Long-term administration of estradiol decreases expression of hepatic lipogenic genes and improves insulin sensitivity in ob/ob mice: a possible mechanism is through direct regulation of signal 
transducer and activator of transcription 3. Mol Endocrinol. 2006;20(6):12871299.

160. Glass CK, Saijo K, Winner B, Marchetto MC, Gage FH. Mechanisms underlying inflammation in neurodegeneration. Cell. 2010;140(6):918-934.

161. Valente T, Dentesano G, Ezquerra M, et al. CCAAT/enhancer binding protein delta is a transcriptional repressor of alpha-synuclein. Cell Death Differ. 2020;27(2):509-524.

162. Li R, Strohmeyer R, Liang Z, Lue LF, Rogers J. CCAAT/enhancer binding protein delta (C/EBPdelta) expression and elevation in Alzheimer's disease. Neurobiol Aging. 2004;25(8):991-999.

163. Wang S-M, Lim S-W, Wang Y-H, et al. Astrocytic CCAAT/Enhancer-binding protein delta contributes to reactive oxygen species formation in neuroinflammation. Redox biology. 2018;16:104-112.

164. Nishioka K, Ohshima S, Umeshita-Sasai M, et al. Enhanced expression and DNA binding activity of two CCAAT/enhancer-binding protein isoforms, C/EBPbeta and C/EBPdelta, in rheumatoid synovium. Arthritis Rheum. 2000;43(7):15911596.

165. Mutschler E, Geisslinger G, Kroemer HK, Ruth P, Schäfer-Korting M. Mutschler Arzneimittelwirkungen: Lehrbuch der Pharmakologie und Toxikologie. Wiss. Verlag-Ges.; 2008.

166. Harris ED, Jr. Rheumatoid arthritis. Pathophysiology and implications for therapy. N Engl J Med. 1990;322(18):1277-1289.

167. Siouti $E$, Andreakos $E$. The many facets of macrophages in rheumatoid arthritis. Biochem Pharmacol. 2019;165:152-169.

168. Alivernini S, Peluso G, Fedele AL, Tolusso B, Gremese E, Ferraccioli G. Tapering and discontinuation of TNF-alpha blockers without disease relapse using ultrasonography as a tool to identify patients with rheumatoid arthritis in clinical and histological remission. Arthritis Res Ther. 2016;18:39.

169. Kinne RW, Brauer R, Stuhlmuller B, Palombo-Kinne E, Burmester GR. Macrophages in rheumatoid arthritis. Arthritis Res. 2000;2(3):189-202.

170. Smolen JS, Aletaha D, Mclnnes IB. Rheumatoid arthritis. Lancet. 2016;388(10055):2023-2038.

171. Kinne RW, Stuhlmuller B, Burmester GR. Cells of the synovium in rheumatoid arthritis. Macrophages. Arthritis Res Ther. 2007;9(6):224.

172. Di Benedetto P, Ruscitti P, Vadasz Z, Toubi E, Giacomelli R. Macrophages with regulatory functions, a possible new therapeutic perspective in autoimmune diseases. Autoimmun Rev. 2019;18(10):102369.

173. Mulherin D, Fitzgerald O, Bresnihan B. Synovial tissue macrophage populations and articular damage in rheumatoid arthritis. Arthritis Rheum. 1996;39(1):115124.

174. Tak PP, Smeets TJ, Daha MR, et al. Analysis of the synovial cell infiltrate in early rheumatoid synovial tissue in relation to local disease activity. Arthritis Rheum. 1997;40(2):217-225.

175. Athanasou NA. Synovial macrophages. Ann Rheum Dis. 1995;54(5):392-394.

176. Martinez FO, Sica A, Mantovani A, Locati M. Macrophage activation and polarization. Front Biosci. 2008;13:453-461. 
177. Chistiakov DA, Myasoedova VA, Revin VV, Orekhov AN, Bobryshev YV. The impact of interferon-regulatory factors to macrophage differentiation and polarization into M1 and M2. Immunobiology. 2018;223(1):101-111.

178. Tardito S, Martinelli G, Soldano S, et al. Macrophage M1/M2 polarization and rheumatoid arthritis: A systematic review. Autoimmun Rev. 2019;18(11):102397.

179. Wang Y, Han CC, Cui D, Li Y, Ma Y, Wei W. Is macrophage polarization important in rheumatoid arthritis? Int Immunopharmacol. 2017;50:345-352.

180. Zhang YH, Lin JX, Vilcek J. Interleukin- 6 induction by tumor necrosis factor and interleukin-1 in human fibroblasts involves activation of a nuclear factor binding to a kappa B-like sequence. Mol Cell Biol. 1990;10(7):3818-3823.

181. Mihara M, Moriya Y, Kishimoto T, Ohsugi Y. Interleukin-6 (IL-6) induces the proliferation of synovial fibroblastic cells in the presence of soluble IL-6 receptor. Br J Rheumatol. 1995;34(4):321-325.

182. Deon D, Ahmed S, Tai K, et al. Cross-talk between IL-1 and IL- 6 signaling pathways in rheumatoid arthritis synovial fibroblasts. The Journal of Immunology. 2001;167(9):5395-5403.

183. Liote F, Boval-Boizard B, Weill D, Kuntz D, Wautier JL. Blood monocyte activation in rheumatoid arthritis: increased monocyte adhesiveness, integrin expression, and cytokine release. Clin Exp Immunol. 1996;106(1):13-19.

184. Adamopoulos I, Sabokbar A, Wordsworth B, Carr A, Ferguson D, Athanasou NA. Synovial fluid macrophages are capable of osteoclast formation and resorption. The Journal of Pathology: A Journal of the Pathological Society of Great Britain and Ireland. 2006;208(1):35-43.

185. Mclnnes IB, Schett G. The pathogenesis of rheumatoid arthritis. N Engl J Med. 2011;365(23):2205-2219.

186. Okamura $\mathrm{Y}$, Watari $\mathrm{M}$, Jerud ES, et al. The extra domain $\mathrm{A}$ of fibronectin activates Toll-like receptor 4. J Biol Chem. 2001;276(13):10229-10233.

187. Park JS, Svetkauskaite D, He Q, et al. Involvement of toll-like receptors 2 and 4 in cellular activation by high mobility group box 1 protein. I Biol Chem. 2004;279(9):7370-7377.

188. Abdollahi-Roodsaz S, Joosten LA, Koenders MI, et al. Stimulation of TLR2 and TLR4 differentially skews the balance of T cells in a mouse model of arthritis. $J$ Clin Invest. 2008;118(1):205-216.

189. Tizaoui K, Naouali A, Kaabachi W, Hamzaoui A, Hamzaoui K. Association of Toll like receptor Asp299Gly with rheumatoid arthritis risk: A systematic review of case-control studies and meta-analysis. Pathology-Research and Practice. 2015;211(3):219-225.

190. Huang Q, Ma Y, Adebayo A, Pope RM. Increased macrophage activation mediated through toll-like receptors in rheumatoid arthritis. Arthritis \& Rheumatism: Official Journal of the American College of Rheumatology. 2007;56(7):2192-2201.

191. Iwahashi M, Yamamura M, Aita T, et al. Expression of Toll-like receptor 2 on CD16+ blood monocytes and synovial tissue macrophages in rheumatoid arthritis. Arthritis \& Rheumatism. 2004;50(5):1457-1467.

192. Joosten LA, Koenders MI, Smeets RL, et al. Toll-like receptor 2 pathway drives streptococcal cell wall-induced joint inflammation: critical role of myeloid differentiation factor 88. The Journal of Immunology. 2003;171(11):6145-6153. 
193. Milman N, Karsh J, Booth RA. Correlation of a multi-cytokine panel with clinical disease activity in patients with rheumatoid arthritis. Clinical biochemistry. 2010;43(16-17):1309-1314.

194. Dalton DK, Pitts-Meek S, Keshav S, Figari IS, Bradley A, Stewart TA. Multiple defects of immune cell function in mice with disrupted interferon-gamma genes. Science. 1993;259(5102):1739-1742.

195. Mantovani A, Garlanda C, Locati M. Macrophage diversity and polarization in atherosclerosis: a question of balance. Arterioscler Thromb Vasc Biol. 2009;29(10):1419-1423.

196. Tsuchiya S, Yamabe M, Yamaguchi Y, Kobayashi Y, Konno T, Tada K. Establishment and characterization of a human acute monocytic leukemia cell line (THP-1). Int J Cancer. 1980;26(2):171-176.

197. Chanput W, Mes JJ, Wichers HJ. THP-1 cell line: an in vitro cell model for immune modulation approach. Int Immunopharmacol. 2014;23(1):37-45.

198. Tanida S, Yoshitomi H, Nishitani K, et al. CCL2O produced in the cytokine network of rheumatoid arthritis recruits CCR6+ mononuclear cells and enhances the production of IL-6. Cytokine. 2009;47(2):112-118.

199. Dhawan P, Richmond A. Role of CXCL1 in tumorigenesis of melanoma. J Leukoc Biol. 2002;72(1):9-18.

200. Lai CS, Lee JH, Ho CT, et al. Rosmanol potently inhibits lipopolysaccharideinduced iNOS and COX-2 expression through downregulating MAPK, NF-kappaB, STAT3 and C/EBP signaling pathways. J Agric Food Chem. 2009;57(22):1099010998.

201. Kuo YC, Lai CS, Wang JM, et al. Differential inhibitory effects of inotilone on inflammatory mediators, inducible nitric oxide synthase and cyclooxygenase-2, in LPS-stimulated murine macrophage. Mol Nutr Food Res. 2009;53(11):13861395.

202. Hanke T, Merk D, Steinhilber D, Geisslinger G, Schubert-Zsilavecz M. Small molecules with anti-inflammatory properties in clinical development. Pharmacol Ther. 2016;157:163-187.

203. Smolen JS, Landewé R, Bijlsma JW, et al. EULAR recommendations for the management of rheumatoid arthritis with synthetic and biological diseasemodifying antirheumatic drugs: 2019 update. Annals of the rheumatic diseases. 2020.

204. Bachmann BJ. Linkage map of Escherichia coli K-7. Microbiological reviews. 1983;47(2):180.

205. Osoegawa K, Woon PY, Zhao B, et al. An improved approach for construction of bacterial artificial chromosome libraries. Genomics. 1998;52(1):1-8.

206. Huang F, Spangler JR, Huang AY. In vivo cloning of up to $16 \mathrm{~kb}$ plasmids in E. coli is as simple as PCR. PloS one. 2017;12(8):e0183974.

207. Holmes DS, Quigley M. A rapid boiling method for the preparation of bacterial plasmids. Analytical biochemistry. 1981;114(1):193-197.

208. Muyrers JP, Zhang Y, Testa G, Stewart AF. Rapid modification of bacterial artificial chromosomes by ET-recombination. Nucleic acids research. 1999;27(6):15551557.

209. Livak KJ, Schmittgen TD. Analysis of relative gene expression data using real-time quantitative PCR and the 2- $\Delta \Delta C T$ method. methods. 2001;25(4):402-408. 
210. Maeß MB, Sendelbach S, Lorkowski S. Selection of reliable reference genes during THP-1 monocyte differentiation into macrophages. BMC molecular biology. 2010;11(1):90.

211. Lytton J, Westlin M, Hanley MR. Thapsigargin inhibits the sarcoplasmic or endoplasmic reticulum Ca-ATPase family of calcium pumps. Journal of Biological Chemistry. 1991;266(26):17067-17071.

212. Tannous BA. Gaussia luciferase reporter assay for monitoring biological processes in culture and in vivo. Nature protocols. 2009;4(4):582.

213. Sui $\mathrm{Y}, \mathrm{Wu} \mathrm{Z}$. Alternative statistical parameter for high-throughput screening assay quality assessment. Journal of biomolecular screening. 2007;12(2):229234.

214. Shiratori H, Feinweber C, Luckhardt S, et al. THP-1 and human peripheral blood mononuclear cell-derived macrophages differ in their capacity to polarize in vitro. Mol Immunol. 2017;88:58-68.

215. Coussens NP, Sittampalam GS, Guha R, et al. Assay Guidance Manual: Quantitative Biology and Pharmacology in Preclinical Drug Discovery. Clin Transl Sci. 2018;11(5):461-470.

216. Daigneault M, Preston JA, Marriott HM, Whyte MK, Dockrell DH. The identification of markers of macrophage differentiation in PMA-stimulated THP1 cells and monocyte-derived macrophages. PLoS One. 2010;5(1):e8668.

217. Lee AS. The ER chaperone and signaling regulator GRP78/BiP as a monitor of endoplasmic reticulum stress. Methods. 2005;35(4):373-381.

218. Grafarend E, Awange J. Linear and Nonlinear Models: Fixed Effects, Random Effects, and Total Least Squares. Springer; 2012.

219. Shiratori H. Establishment of Systemic in Vitro Screening System to Identify Modulatory Compounds for Human Macrophage Polarization, Johann Wolfgang Goethe-Universität Frankfurt am Main; 2017.

220. Odero MD, Zeleznik-Le NJ, Chinwalla V, Rowley JD. Cytogenetic and molecular analysis of the acute monocytic leukemia cell line THP-1 with an MLL-AF9 translocation. Genes, Chromosomes and Cancer. 2000;29(4):333-338.

221. Spano A, Barni S, Sciola L. PMA withdrawal in PMA-treated monocytic THP-1 cells and subsequent retinoic acid stimulation, modulate induction of apoptosis and appearance of dendritic cells. Cell Prolif. 2013;46(3):328-347.

222. Sekhon-Loodu S, Ziaullah, Rupasinghe HP. Docosahexaenoic acid ester of phloridzin inhibit lipopolysaccharide-induced inflammation in THP-1 differentiated macrophages. Int Immunopharmacol. 2015;25(1):199-206.

223. Schwende $H$, Fitzke $E$, Ambs $P$, Dieter $P$. Differences in the state of differentiation of THP-1 cells induced by phorbol ester and 1,25-dihydroxyvitamin D3. J Leukoc Biol. 1996;59(4):555-561.

224. Kasper D, Fauci A, Longo D. Harrisons Principle Of Internal Medicine. New York McGraw. In: Hill publishing; 2005.

225. Cousins RJ, Blanchard RK, Popp MP, et al. A global view of the selectivity of zinc deprivation and excess on genes expressed in human THP-1 mononuclear cells. Proc Natl Acad Sci U S A. 2003;100(12):6952-6957.

226. Rogers PD, Thornton J, Barker KS, et al. Pneumolysin-dependent and independent gene expression identified by CDNA microarray analysis of THP-1 
human mononuclear cells stimulated by Streptococcus pneumoniae. Infect Immun. 2003;71(4):2087-2094.

227. Gottlicher M, Minucci S, Zhu P, et al. Valproic acid defines a novel class of HDAC inhibitors inducing differentiation of transformed cells. EMBO J. 2001;20(24):6969-6978.

228. Xu WS, Parmigiani RB, Marks PA. Histone deacetylase inhibitors: molecular mechanisms of action. Oncogene. 2007;26(37):5541-5552.

229. Tough DF, Tak PP, Tarakhovsky A, Prinjha RK. Epigenetic drug discovery: breaking through the immune barrier. Nat Rev Drug Discov. 2016;15(12):835-853.

230. Mohammadi A, Sharifi A, Pourpaknia R, Mohammadian S, Sahebkar A. Manipulating macrophage polarization and function using classical HDAC inhibitors: Implications for autoimmunity and inflammation. Crit Rev Oncol Hematol. 2018;128:1-18.

231. Lehrmann H, Pritchard LL, Harel-Bellan A. Histone acetyltransferases and deacetylases in the control of cell proliferation and differentiation. Adv Cancer Res. 2002;86:41-65.

232. Nishimura $Y$, Sasagawa $S$, Ariyoshi $M$, et al. Systems pharmacology of adiposity reveals inhibition of EP300 as a common therapeutic mechanism of caloric restriction and resveratrol for obesity. Front Pharmacol. 2015;6:199.

233. Klein K. Bromodomain protein inhibition: a novel therapeutic strategy in rheumatic diseases. RMD open. 2018;4(2):e000744.

234. Filippakopoulos $\mathrm{P}$, Picaud S, Mangos M, et al. Histone recognition and large-scale structural analysis of the human bromodomain family. Cell. 2012;149(1):214231.

235. Zeng L, Zhou MM. Bromodomain: an acetyl-lysine binding domain. FEBS Lett. 2002;513(1):124-128.

236. Hong L, Schroth GP, Matthews HR, Yau P, Bradbury EM. Studies of the DNA binding properties of histone $\mathrm{H} 4$ amino terminus. Thermal denaturation studies reveal that acetylation markedly reduces the binding constant of the $\mathrm{H} 4$ "tail" to DNA. J Biol Chem. 1993;268(1):305-314.

237. Fujisawa $T$, Filippakopoulos $P$. Functions of bromodomain-containing proteins and their roles in homeostasis and cancer. Nat Rev Mol Cell Biol. 2017;18(4):246262.

238. Lamonica JM, Deng W, Kadauke S, et al. Bromodomain protein Brd3 associates with acetylated GATA1 to promote its chromatin occupancy at erythroid target genes. Proc Natl Acad Sci U S A. 2011;108(22):E159-168.

239. Huang B, Yang XD, Zhou MM, Ozato K, Chen LF. Brd4 coactivates transcriptional activation of NF-kappaB via specific binding to acetylated RelA. Mol Cell Biol. 2009;29(5):1375-1387.

240. Blander G, Guarente L. The Sir2 family of protein deacetylases. Annu Rev Biochem. 2004;73:417-435.

241. Bhalla KN. Epigenetic and chromatin modifiers as targeted therapy of hematologic malignancies. J Clin Oncol. 2005;23(17):3971-3993.

242. Marks PA, Dokmanovic M. Histone deacetylase inhibitors: discovery and development as anticancer agents. Expert Opin Investig Drugs. 2005;14(12):1497-1511. 
243. Gregoretti IV, Lee YM, Goodson HV. Molecular evolution of the histone deacetylase family: functional implications of phylogenetic analysis. $J \mathrm{Mol} \mathrm{Biol}$. 2004;338(1):17-31.

244. Gao L, Cueto MA, Asselbergs F, Atadja P. Cloning and functional characterization of HDAC11, a novel member of the human histone deacetylase family. $J$ Biol Chem. 2002;277(28):25748-25755.

245. Leus NG, Zwinderman MR, Dekker FJ. Histone deacetylase 3 (HDAC 3) as emerging drug target in NF-kappaB-mediated inflammation. Curr Opin Chem Biol. 2016;33:160-168.

246. Glozak MA, Sengupta N, Zhang X, Seto E. Acetylation and deacetylation of nonhistone proteins. Gene. 2005;363:15-23.

247. Iwabata H, Yoshida M, Komatsu Y. Proteomic analysis of organ-specific posttranslational lysine-acetylation and -methylation in mice by use of antiacetyllysine and -methyllysine mouse monoclonal antibodies. Proteomics. 2005;5(18):4653-4664.

248. Kim SC, Sprung R, Chen $Y$, et al. Substrate and functional diversity of lysine acetylation revealed by a proteomics survey. Mol Cell. 2006;23(4):607-618.

249. Salvador LA, Luesch H. Discovery and mechanism of natural products as modulators of histone acetylation. Curr Drug Targets. 2012;13(8):1029-1047.

250. Hull EE, Montgomery MR, Leyva KJ. HDAC Inhibitors as Epigenetic Regulators of the Immune System: Impacts on Cancer Therapy and Inflammatory Diseases. Biomed Res Int. 2016;2016:8797206.

251. Eckschlager T, Plch J, Stiborova M, Hrabeta J. Histone Deacetylase Inhibitors as Anticancer Drugs. Int J Mol Sci. 2017;18(7).

252. Greer CB, Tanaka Y, Kim YJ, et al. Histone Deacetylases Positively Regulate Transcription through the Elongation Machinery. Cell Rep. 2015;13(7):14441455.

253. Grabiec AM, Korchynskyi O, Tak PP, Reedquist KA. Histone deacetylase inhibitors suppress rheumatoid arthritis fibroblast-like synoviocyte and macrophage IL-6 production by accelerating mRNA decay. Ann Rheum Dis. 2012;71(3):424-431.

254. Filippakopoulos P, Knapp S. Targeting bromodomains: epigenetic readers of lysine acetylation. Nature reviews Drug discovery. 2014;13(5):337-356.

255. Mirguet O, Lamotte Y, Donche F, et al. From ApoA1 upregulation to BET family bromodomain inhibition: discovery of I-BET151. Bioorganic \& medicinal chemistry letters. 2012;22(8):2963-2967.

256. Dawson MA, Prinjha RK, Dittmann A, et al. Inhibition of BET recruitment to chromatin as an effective treatment for MLL-fusion leukaemia. Nature. 2011;478(7370):529-533.

257. Seal J, Lamotte $Y$, Donche $F$, et al. Identification of a novel series of BET family bromodomain inhibitors: binding mode and profile of I-BET151 (GSK1210151A). Bioorganic \& medicinal chemistry letters. 2012;22(8):2968-2972.

258. Hewings DS, Wang M, Philpott $M$, et al. 3, 5-dimethylisoxazoles act as acetyllysine-mimetic bromodomain ligands. Journal of medicinal chemistry. 2011;54(19):6761-6770.

259. Bamborough P, Diallo $H$, Goodacre JD, et al. Fragment-based discovery of bromodomain inhibitors part 2: optimization of phenylisoxazole sulfonamides. Journal of medicinal chemistry. 2012;55(2):587-596. 
260. Nicodeme $E$, Jeffrey $K L$, Schaefer $U$, et al. Suppression of inflammation by a synthetic histone mimic. Nature. 2010;468(7327):1119-1123.

261. Klein K, Kabala PA, Grabiec AM, et al. The bromodomain protein inhibitor IBET151 suppresses expression of inflammatory genes and matrix degrading enzymes in rheumatoid arthritis synovial fibroblasts. Annals of the rheumatic diseases. 2016;75(2):422-429.

262. Kempen $\mathrm{H}$. Use of a thienotriazolodiazephine to increase apolipoprotein $\mathrm{Al}$ levels. In: Google Patents; 1998.

263. Kempen HJ, Bellus D, Fedorov O, et al. Stimulation of hepatic apolipoprotein Al production by novel thieno-triazolodiazepines: roles of the classical benzodiazepine receptor, PAF receptor, and bromodomain binding. In: SAGE Publications Sage UK: London, England; 2013.

264. Zanotti I, Maugeais C, Pedrelli M, et al. The thienotriazolodiazepine Ro 11-1464 increases plasma apoA-I and promotes reverse cholesterol transport in human apoA-I transgenic mice. British journal of pharmacology. 2011;164(6):16421651.

265. Richon VM, Webb Y, Merger R, et al. Second generation hybrid polar compounds are potent inducers of transformed cell differentiation. Proc Natl Acad Sci U S A. 1996;93(12):5705-5708.

266. Mann BS, Johnson JR, Cohen MH, Justice R, Pazdur R. FDA approval summary: vorinostat for treatment of advanced primary cutaneous T-cell lymphoma. Oncologist. 2007;12(10):1247-1252.

267. Leoni F, Zaliani A, Bertolini G, et al. The antitumor histone deacetylase inhibitor suberoylanilide hydroxamic acid exhibits antiinflammatory properties via suppression of cytokines. Proc Natl Acad Sci U S A. 2002;99(5):2995-3000.

268. Lohman RJ, Iyer A, Fairlie TJ, et al. Differential Anti-inflammatory Activity of HDAC Inhibitors in Human Macrophages and Rat Arthritis. J Pharmacol Exp Ther. 2016;356(2):387-396.

269. Choo QY, Ho PC, Tanaka Y, Lin HS. The histone deacetylase inhibitors MS-275 and SAHA suppress the p38 mitogen-activated protein kinase signaling pathway and chemotaxis in rheumatoid arthritic synovial fibroblastic E11 cells. Molecules. 2013;18(11):14085-14095.

270. Tsuji N, Kobayashi M, Nagashima K, Wakisaka Y, Koizumi K. A new antifungal antibiotic, trichostatin. J Antibiot (Tokyo). 1976;29(1):1-6.

271. Lobera M, Madauss KP, Pohlhaus DT, et al. Selective class Ila histone deacetylase inhibition via a nonchelating zinc-binding group. Nat Chem Biol. 2013;9(5):319325.

272. Vigushin DM, Ali S, Pace PE, et al. Trichostatin A is a histone deacetylase inhibitor with potent antitumor activity against breast cancer in vivo. Clin Cancer Res. 2001;7(4):971-976.

273. Grabiec AM, Krausz S, de Jager W, et al. Histone deacetylase inhibitors suppress inflammatory activation of rheumatoid arthritis patient synovial macrophages and tissue. J Immunol. 2010;184(5):2718-2728.

274. Newman JR, Keating AE. Comprehensive identification of human bZIP interactions with coiled-coil arrays. Science. 2003;300(5628):2097-2101. 
275. Tsukada J, Saito K, Waterman WR, Webb AC, Auron PE. Transcription factors NFIL6 and CREB recognize a common essential site in the human prointerleukin 1 beta gene. Mol Cell Biol. 1994;14(11):7285-7297.

276. Diehl JA, Hannink M. Identification of a C/EBP-Rel complex in avian lymphoid cells. Mol Cell Biol. 1994;14(10):6635-6646.

277. Dooher JE, Paz-Priel I, Houng S, Baldwin AS, Jr., Friedman AD. C/EBPalpha, C/EBPalpha oncoproteins, or C/EBPbeta preferentially bind NF-kappaB p50 compared with p65, focusing therapeutic targeting on the C/EBP:p50 interaction. Mol Cancer Res. 2011;9(10):1395-1405.

278. Plevy SE, Gemberling JH, Hsu S, Dorner AJ, Smale ST. Multiple control elements mediate activation of the murine and human interleukin 12 p40 promoters: evidence of functional synergy between C/EBP and Rel proteins. Mol Cell Biol. 1997;17(8):4572-4588.

279. Agrawal A, Cha-Molstad H, Samols D, Kushner I. Transactivation of C-reactive protein by IL- 6 requires synergistic interaction of CCAAT/enhancer binding protein beta (C/EBP beta) and Rel p50. J Immunol. 2001;166(4):2378-2384.

280. Romagnoli L, Wollebo HS, Deshmane SL, et al. Modulation of JC virus transcription by C/EBPbeta. Virus Res. 2009;146(1-2):97-106.

281. Matsusaka T, Fujikawa K, Nishio Y, et al. Transcription factors NF-IL6 and NFkappa B synergistically activate transcription of the inflammatory cytokines, interleukin 6 and interleukin 8. Proc Natl Acad Sci U S A. 1993;90(21):1019310197.

282. Stein B, Cogswell PC, Baldwin AS, Jr. Functional and physical associations between NF-kappa B and C/EBP family members: a Rel domain-bZIP interaction. Mol Cell Biol. 1993;13(7):3964-3974.

283. Barrett E, Brothers S, Wahlestedt C, Beurel E. I-BET151 selectively regulates IL-6 production. Biochimica et Biophysica Acta (BBA)-Molecular Basis of Disease. 2014;1842(9):1549-1555.

284. de Bont N, Netea MG, Rovers C, et al. LPS-induced cytokine production and expression of LPS-receptors by peripheral blood mononuclear cells of patients with familial hypercholesterolemia and the effect of HMG-CoA reductase inhibitors. Atherosclerosis. 1998;139(1):147-152.

285. Chan CH, Fang C, Qiao Y, Yarilina A, Prinjha RK, Ivashkiv LB. BET bromodomain inhibition suppresses transcriptional responses to cytokine-Jak-STAT signaling in a gene-specific manner in human monocytes. European journal of immunology. 2015;45(1):287-297.

286. Belkina AC, Denis GV. BET domain co-regulators in obesity, inflammation and cancer. Nature reviews Cancer. 2012;12(7):465-477.

287. Dey A, Chitsaz F, Abbasi A, Misteli T, Ozato K. The double bromodomain protein Brd4 binds to acetylated chromatin during interphase and mitosis. Proceedings of the National Academy of Sciences. 2003;100(15):8758-8763.

288. Yang Z, Yik JH, Chen R, et al. Recruitment of P-TEFb for stimulation of transcriptional elongation by the bromodomain protein Brd4. Molecular cell. 2005;19(4):535-545.

289. Brès V, Yoh SM, Jones KA. The multi-tasking P-TEFb complex. Current opinion in cell biology. 2008;20(3):334-340. 
290. Liu W, Ma Q, Wong K, et al. Brd4 and JMJD6-associated anti-pause enhancers in regulation of transcriptional pause release. Cell. 2013;155(7):1581-1595.

291. Kanno T, Kanno Y, LeRoy G, et al. BRD4 assists elongation of both coding and enhancer RNAs by interacting with acetylated histones. Nature structural \& molecular biology. 2014;21(12):1047.

292. Zou Z, Huang B, Wu X, et al. Brd4 maintains constitutively active $\mathrm{nf}-\mathrm{k} \mathrm{b}$ in cancer cells by binding to acetylated rela. Oncogene. 2014;33(18):2395-2404.

293. Belkina AC, Nikolajczyk BS, Denis GV. BET protein function is required for inflammation: Brd2 genetic disruption and BET inhibitor JQ1 impair mouse macrophage inflammatory responses. The Journal of Immunology. 2013;190(7):3670-3678.

294. Chen W, Wu Y, Lu Q, Wang S, Xing D. Endogenous ApoA-I expression in macrophages: a potential target for protection against atherosclerosis. Clinica Chimica Acta. 2020.

295. Mogilenko DA, Orlov SV, Trulioff AS, et al. Endogenous apolipoprotein AI stabilizes ATP-binding cassette transporter A1 and modulates Toll-like receptor 4 signaling in human macrophages. The FASEB Journal. 2012;26(5):2019-2030.

296. Shavva VS, Mogilenko DA, Nekrasova EV, et al. Tumor necrosis factor $\alpha$ stimulates endogenous apolipoprotein $\mathrm{Al}$ expression and secretion by human monocytes and macrophages: Role of MAP-kinases, NF-KB, and nuclear receptors PPAR $\alpha$ and LXRs. Molecular and cellular biochemistry. 2018;448(12):211-223.

297. Bogomolova A, Shavva V, Nikitin A, et al. Hypoxia as a factor involved in the regulation of the apoA-1, $A B C A 1$, and complement $C 3$ gene expression in human macrophages. Biochemistry (Moscow). 2019;84(5):529-539.

298. Bursill CA, Castro ML, Beattie DT, et al. High-density lipoproteins suppress chemokines and chemokine receptors in vitro and in vivo. Arteriosclerosis, thrombosis, and vascular biology. 2010;30(9):1773-1778.

299. Choo QY, Ho PC, Tanaka Y, Lin HS. Histone deacetylase inhibitors MS-275 and SAHA induced growth arrest and suppressed lipopolysaccharide-stimulated NFkappaB p65 nuclear accumulation in human rheumatoid arthritis synovial fibroblastic E11 cells. Rheumatology (Oxford). 2010;49(8):1447-1460.

300. Yuan ZL, Guan YJ, Chatterjee D, Chin YE. Stat3 dimerization regulated by reversible acetylation of a single lysine residue. Science. 2005;307(5707):269273.

301. Kiernan R, Bres V, Ng RW, et al. Post-activation turn-off of NF-kappa B-dependent transcription is regulated by acetylation of p65. J Biol Chem. 2003;278(4):27582766.

302. Chen L, Fischle W, Verdin E, Greene WC. Duration of nuclear NF-kappaB action regulated by reversible acetylation. Science. 2001;293(5535):1653-1657.

303. Kim Y, Kim K, Park D, et al. Histone deacetylase 3 mediates allergic skin inflammation by regulating expression of MCP1 protein. J Biol Chem. 2012;287(31):25844-25859.

304. Sun L, Telles E, Karl M, et al. Loss of HDAC11 ameliorates clinical symptoms in a multiple sclerosis mouse model. Life Sci Alliance. 2018;1(5):e201800039.

305. Han SB, Lee JK. Anti-inflammatory effect of Trichostatin-A on murine bone marrow-derived macrophages. Arch Pharm Res. 2009;32(4):613-624. 
306. Aung HT, Schroder K, Himes SR, et al. LPS regulates proinflammatory gene expression in macrophages by altering histone deacetylase expression. FASEB J. 2006;20(9):1315-1327.

307. Hepp MI, Escobar D, Farkas C, et al. A Trichostatin A (TSA)/Sp1-mediated mechanism for the regulation of SALL2 tumor suppressor in Jurkat T cells. Biochim Biophys Acta Gene Regul Mech. 2018.

308. Lee KW, Lee Y, Kwon HJ, Kim DS. Sp1-associated activation of macrophage inflammatory protein-2 promoter by CpG-oligodeoxynucleotide and lipopolysaccharide. Cell Mol Life Sci. 2005;62(2):188-198.

309. Liu YW, Wang SA, Hsu TY, Chen TA, Chang WC, Hung JJ. Inhibition of LPS-induced C/EBP delta by trichostatin A has a positive effect on LPS-induced cyclooxygenase 2 expression in RAW264.7 cells. J Cell Biochem. 2010;110(6):1430-1438.

310. Zhang $Q$, Yang $F$, Li X, et al. Trichostatin A inhibits inflammation in phorbol myristate acetateinduced macrophages by regulating the acetylation of histone and/or nonhistone proteins. Mol Med Rep. 2016;13(1):845-852.

311. Hu XL, Zhang X, Li Q, Qiu SF, Mei RH. [Effects of trichostatin A on the expressions of inflammatory cytokines and toll-like receptor 4 and the acetylation of nuclear factor-kappaB induced by lipopolysaccharide in macrophage]. Sheng Li Xue Bao. 2012;64(6):651-656.

312. Laribee RN, Klemsz MJ. Loss of PU.1 expression following inhibition of histone deacetylases. J Immunol. 2001;167(9):5160-5166.

313. Cabanel M, Brand C, Oliveira-Nunes MC, et al. Epigenetic Control of Macrophage Shape Transition towards an Atypical Elongated Phenotype by Histone Deacetylase Activity. PLoS One. 2015;10(7):e0132984.

314. Thangavel J, Samanta S, Rajasingh S, et al. Epigenetic modifiers reduce inflammation and modulate macrophage phenotype during endotoxemiainduced acute lung injury. J Cell Sci. 2015;128(16):3094-3105.

315. Klein K, Ospelt C, Gay S. Epigenetic contributions in the development of rheumatoid arthritis. Arthritis Res Ther. 2012;14(6):227.

316. Klein K, Gay S. Epigenetics in rheumatoid arthritis. Curr Opin Rheumatol. 2015;27(1):76-82.

317. Bartok B, Firestein GS. Fibroblast-like synoviocytes: key effector cells in rheumatoid arthritis. Immunol Rev. 2010;233(1):233-255.

318. Drexler SK, Kong PL, Wales J, Foxwell BM. Cell signalling in macrophages, the principal innate immune effector cells of rheumatoid arthritis. Arthritis Res Ther. 2008;10(5):216.

319. Urnov FD, Wolffe AP. Chromatin remodeling and transcriptional activation: the cast (in order of appearance). Oncogene. 2001;20(24):2991-3006.

320. Vidler LR, Brown N, Knapp S, Hoelder S. Druggability analysis and structural classification of bromodomain acetyl-lysine binding sites. I Med Chem. 2012;55(17):7346-7359.

321. Kim JY, Lee EY, Park JK, Song YW, Kim JR, Cho KH. Patients with Rheumatoid Arthritis Show Altered Lipoprotein Profiles with Dysfunctional High-Density Lipoproteins that Can Exacerbate Inflammatory and Atherogenic Process. PLoS One. 2016;11(10):e0164564. 
322. Parveen S, Jacob R, Rajasekhar L, Srinivasa C, Mohan IK. Serum Lipid Alterations in Early Rheumatoid Arthritis Patients on Disease Modifying Anti Rheumatoid Therapy. Indian J Clin Biochem. 2017;32(1):26-32.

323. Chen H, Pan J, Wang JD, Liao QM, Xia XR. Suberoylanilide Hydroxamic Acid, an Inhibitor of Histone Deacetylase, Induces Apoptosis in Rheumatoid Arthritis Fibroblast-Like Synoviocytes. Inflammation. 2016;39(1):39-46.

324. Zhang Y, Zhang B. Trichostatin A, an Inhibitor of Histone Deacetylase, Inhibits the Viability and Invasiveness of Hypoxic Rheumatoid Arthritis Fibroblast-Like Synoviocytes via PI3K/Akt Signaling. J Biochem Mol Toxicol. 2016;30(4):163-169.

325. Jungel A, Baresova V, Ospelt $C$, et al. Trichostatin A sensitises rheumatoid arthritis synovial fibroblasts for TRAIL-induced apoptosis. Ann Rheum Dis. 2006;65(7):910-912.

326. Hu H-M, Baer M, Williams SC, Johnson PF, Schwartz RC. Redundancy of C/EBP $\alpha$,$\beta$, and- $\delta$ in supporting the lipopolysaccharide-induced transcription of IL- 6 and monocyte chemoattractant protein-1. The Journal of Immunology. 1998;160(5):2334-2342.

327. Borrelli S, Testoni B, Callari M, et al. Reciprocal regulation of p63 by C/EBP delta in human keratinocytes. BMC molecular biology. 2007;8(1):85.

328. Zhang K, Kaufman RJ. From endoplasmic-reticulum stress to the inflammatory response. Nature. 2008;454(7203):455-462.

329. Qosa H, Mohamed LA, Al Rihani SB, et al. High-throughput screening for identification of blood-brain barrier integrity enhancers: $A$ drug repurposing opportunity to rectify vascular amyloid toxicity. Journal of Alzheimer's Disease. 2016;53(4):1499-1516.

330. Li Y, McGreal S, Zhao J, et al. A cell-based quantitative high-throughput image screening identified novel autophagy modulators. Pharmacological research. 2016;110:35-49.

331. Eriksson A, Österroos A, Hassan S, et al. Drug screen in patient cells suggests quinacrine to be repositioned for treatment of acute myeloid leukemia. Blood cancer journal. 2015;5(4):e307-e307.

332. Poksay KS, Sheffler DJ, Spilman P, et al. Screening for Small Molecule Inhibitors of Statin-Induced APP C-terminal Toxic Fragment Production. Frontiers in pharmacology. 2017;8:46.

333. Yi NY, He Q, Caligan TB, et al. Development of a cell-based fluorescence polarization biosensor using preproinsulin to identify compounds that alter insulin granule dynamics. Assay and drug development technologies. 2015;13(9):558-569.

334. Truong M, Monahan LG, Carter DA, Charles IG. Repurposing drugs to fast-track therapeutic agents for the treatment of cryptococcosis. PeerJ. 2018;6:e4761. 


\section{Supplement}

\subsection{Structure and regulation of CEBPD target promoter}

\subsubsection{Sequencing of CEBPD-encoding region on BAC clone CH17-293N3}

The obtained BAC clone $\mathrm{CH} 17-293 \mathrm{~N} 3$ was verified via partial sequencing (chapter 2.23) using following sequencing primers: BAC_PCR_7, BAC_PCR_2, and BAC_PCR_1 (Table 2.11). Sequencing results of CEBPD gene are shown in Figure 8-1.

\section{APRE}

ggagtgtcat tcccagcagc_gcagcgcagg ccggcccggg ccggggaggg agcagcagcg cgggcaccct cctgcaacgt defined CEBPD promoter

gggctggggg tccccagggc cggggagggg gccccgggga ggaactgaga gggggcgcgc cccgggaagg ctcggggcgg $S P 1$ ctccgggggg ctcccagggc gcccccgccc cttcccccgc ggccccgggg cgcccccgcg gtgccggagt cggggegggg cgtgCRE TATA-box TSS 5' UTR cacgtc agccggggct agaaaaggcg gcggggctgg gcccagccag gtgacagcct cgcttggacg cagagcccgg cccgac-

gccg cc atgagcgc cgcgctcttc agcctggacg gcccggcgcg cggcgcgccc tggcctgcgg agcctgcgcc protein coding region

cttctacgaa ccgggccggg cgggcaagcc gggccgcggg gccgagccag gggccctagg cgagccaggc gccgccgcce ccgccatgta cgacgacgag agcgccatcg acttcagcge ctacatcgac tccatggccg ccgtgcccac cctggagctg tgccacgacg agctcttcgc cgacctcttc aacagcaatc acaaggcggg cggcgcgggg ccctggage ttcttccogg cggccccgcg cgcccettgg gcccgggecc tgccgctccc cgcctgctca agcgcgagcc cgactgggge gacggcgacg cgccoggctc gctgttgcce gcgcaggtgg ccgcgtgcgc acagaccgtg gtgagcttgg cggccgcagg gcagcccacc ccgcccacgt cgccggagcc gccgcgcagc agcccaggc agaccccoge geccggccce gcccgggaga agagcgccgg caagagggge ccggaccgcg gcagccccga gtaccggcag cggcgcgagc gcaacaacat cgccgtgcgc aagagccgcg acaaggccaa gcggcgcaac caggagatgc agcagaagtt ggtggagctg tcggctgaga acgagaagct gcaccagcge gtggagcagc tcacgcggga cctggccgge ctccggcagt tcttcaagca gctgcccagc 
Supplement

3' UTR

ccgccettcc tgccggccgc cgggacagca gactgccggtaa cgcgcggc cggggcggga gagactcagc aac-

gacccat acctcagacc cgacggcccg gagcggagcg cgccctgccc tggcgcagcc agagccgccg ggtgcccgct gcag-

tttctt gggacatagg agcgcaaaga agctacagcc tggacttacc accactaaac tgcgagagaa gctaaacgtg tttattttcc

cttaaattat ttttgtaatg gtagcttttt ctacatctta ctcctgttga tgcagctaag gtacatttgt aaaaagaaaa aaaac-

cagac ttttcagaca aaccctttgt attgtagata agaggaaaag actgagcatg ctcacttttt tatattaatt tttacagtat

ttgtaagaat aaagcagcat ttgaaatcgc

Figure 8-1: Sequencing results of CEBPD encoding region expressed on BAC clone CH17-293N3. Human CEBPD gene (1252 nt) contains 5' UTR (brawn letters, $43 \mathrm{nt}$ ), protein coding region (green italics, $810 \mathrm{nt}$ ), and 3' UTR (brawn letters, $398 \mathrm{nt}$ ). The upstream CEBPD promoter region contains binding sites (bold, underlined letters) for regulatory transcription factors STAT3 (APRE), SP1 (SP1), and CREB (CRE), as well as TATA-box, and transcription start site (TSS). The defined CEBPD promoter (blue underline, $332 \mathrm{nt}$ ), used in multi-gene-reporter cassette 1.0, also includes the $5{ }^{`}$ UTR. CEPBD encoding regions highlighted in gray were confirmed by sequencing. Sequenced regions, which are located adjacent to the shown CEBPD region are not displayed here. 


\subsubsection{Transcriptional regulation of CEBPD}

Expression of C/EBPS TF is mainly regulated at the level of gene transcription initiation (cf. chapter 1.3.1.3). Proximal CEBPD promoter contains known TF binding sites, which functionality is shown in human cells (SP1, CREB, and STAT3). ${ }^{36}$ However, the existence and the functionality of NF-kB binding site is only shown in murine cebpd promoter $^{71}$ Although human CEBPD promoter contains experimentally confirmed ATF3 binding site, ${ }^{10 *}$ its exact location is unknown in human cells. I proposed the location of NF-kB and ATF3 TF binding sites in human CEBPD gene by overlapping of human CEBPD and murine cebpd genes sequences (Fig.8-2), which are similar in their proximal promoter regions.

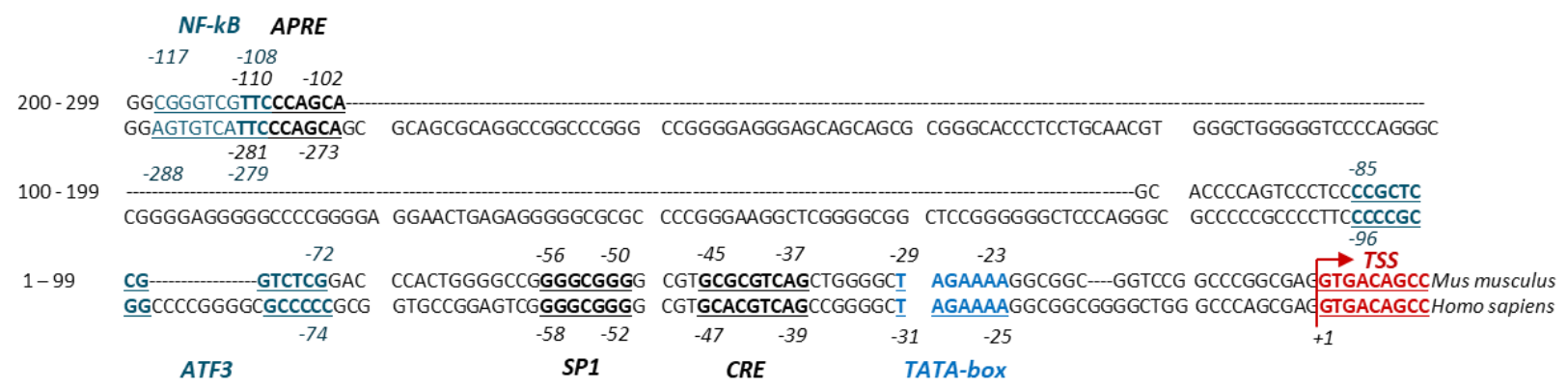

Figure 8-2: Alignment of human CEBPD and murine cebpd promoter sequences. Besides of the TATA-box (blue) and TSS (red), human CEBPD and murine cebpd promoter regions contain TF binding sites SP1, CRE, and APRE, which functionality is confirmed in both species. The murine cebpd promoter also contains functional binding sites of NF-kB (-117/-108) and ATF3 (-85/-72) regulatory TFs (petrol), which functionality is shown only in murine M $\varphi$. In human CEBPD promoter, the location of NF-kB (-288/-279) and ATF3 $(-96 /-74)$ binding sites were proposed by alignment of both promoter sequences. The indicated positions in human CEBPD (numbers below) and murine cebpd (numbers above) promoters refer to the reported TSS for the murine gene.

10* CEBPD gene, ENCODE transcription factor targets. Harmonizome internet site. http://amp.pharm.mssm.edu/Harmonizome/gene/CEBPD. Accessed April 26, 2020. 


\subsection{Characterization of SEAP and eGFP reporter assays}

\subsubsection{Characterization of colorimetric SEAP assay}

In colorimetric assay (Fig.8-3A), SEAP utilizes 4-NPP (4-nitrophenylphosphate) substrate generating 4-nitrohenolat, a yellow product that is detected by absorbance measurement at $405 \mathrm{~nm}$. Colorimetric SEAP assay displayed a limited assay sensitivity (Fig. 8-3B), low, over three 10-fold dilutions, broadness of linear range (Fig.8-3C), and high assay repeat- and reproducibility for both, undiluted and diluted SEAP-containing samples (Fig.8-3D).

A

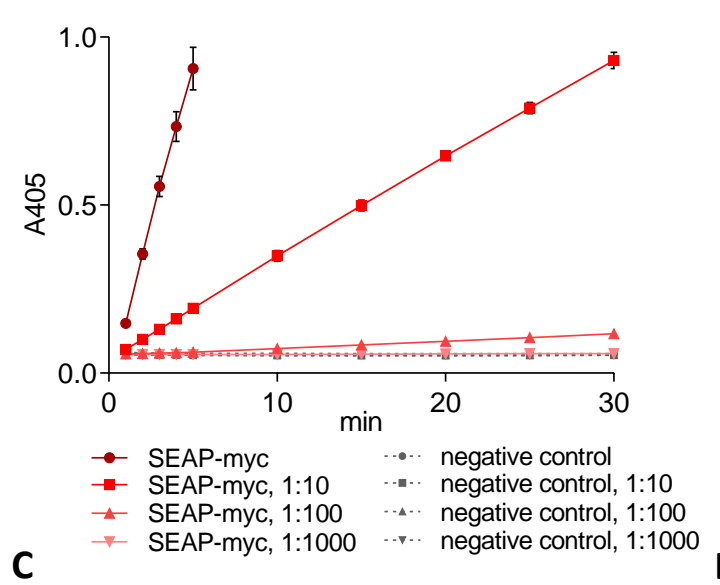

SEAP assay, linearity

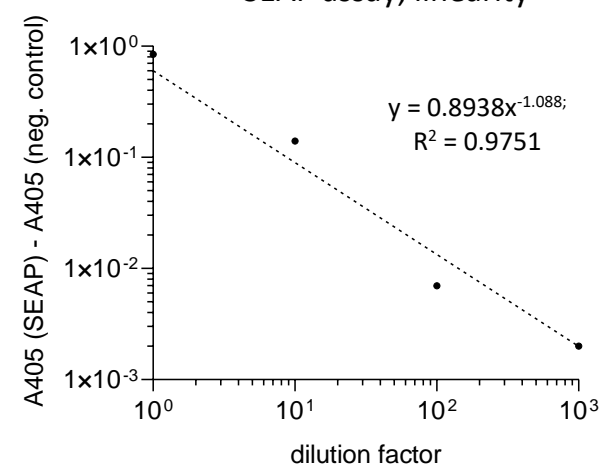

B

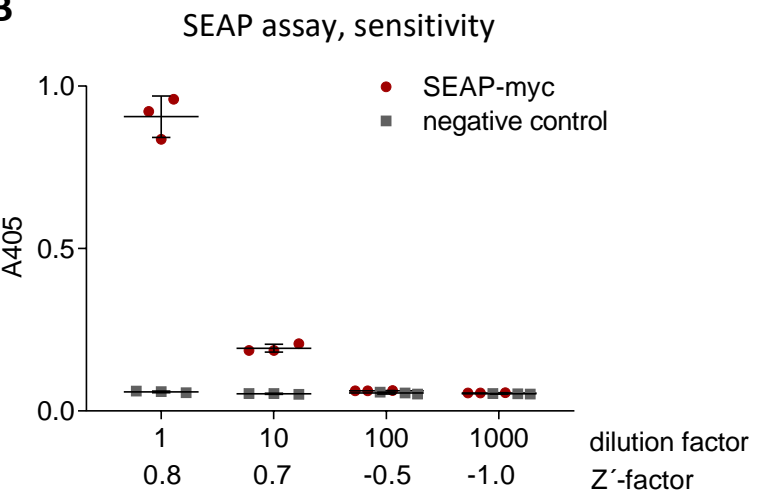

\begin{tabular}{lcc} 
& repeatability & reproducibility \\
\cline { 2 - 3 } sample ID & intra-assay & inter-assay \\
& $\mathrm{CV}(\%)$ & $\mathrm{CV}(\%)$ \\
\hline SEAP-myc & 3.51 & 7.01 \\
SEAP-myc 1:10 & 5.36 & 6.28 \\
SEAP-myc 1:100 & 2.66 & 0.93 \\
SEAP-myc 1:1,000 & 3.76 & 1.04 \\
negative control & 8.67 & 4.48
\end{tabular}

Figure 8-3: Characterization of the colorimetric SEAP assay. SEAP-myc enzymatic activity was measured in undiluted and up to 1,000-fold diluted cell culture supernatants of corresponding HEK293T reporter cells, $24 \mathrm{~h}$ after cell seeding. Cell culture supernatant of HEK293T cells stably transfected with backbone were utilized as negative control. A: Kinetics of enzymatic reaction. SEAP enzymatic signal was measured for each of the dilutions for 30 minutes after substrate addition. Each point represents average signal \pm SD of three independent experiments. B: Determination of assay sensitivity. The highest dilution factor at which $Z^{\prime}$-factor is $\geq 0.5$ is 10 . Data represent mean $\pm S D$ of three independent experiments. C: Determination of assay linearity. Colorimetric SEAP assay was linear $\left(R^{2}=0.9751\right)$ over three 10 -fold dilutions. Each point represents average signal of three independent experiments, detected five minutes after substrate addition. D: Determination of assay repeatability and reproducibility. The intra-assay and inter-assay CVs were determined between the three technical replicates or the three independent experiments, respectively. A405: absorbance at $405 \mathrm{~nm}$. 


\subsubsection{Characterization of fluorescent SEAP assay}

In florescent assay (Fig.8-4A), SEAP dephosphorylates MUP (4-methylumbelliferyl phosphate) substrate generating 4-MU (4-methylumbelliferone), a highly fluorescent and stable product. Fluorescent SEAP assay showed a moderate assay sensitivity (Fig.8-4B), was linear over four 10-fold dilutions (Fig.8-4C), and displayed high assay repeatability and reproducibility for up to 100-fold diluted samples (Fig.8-4D).
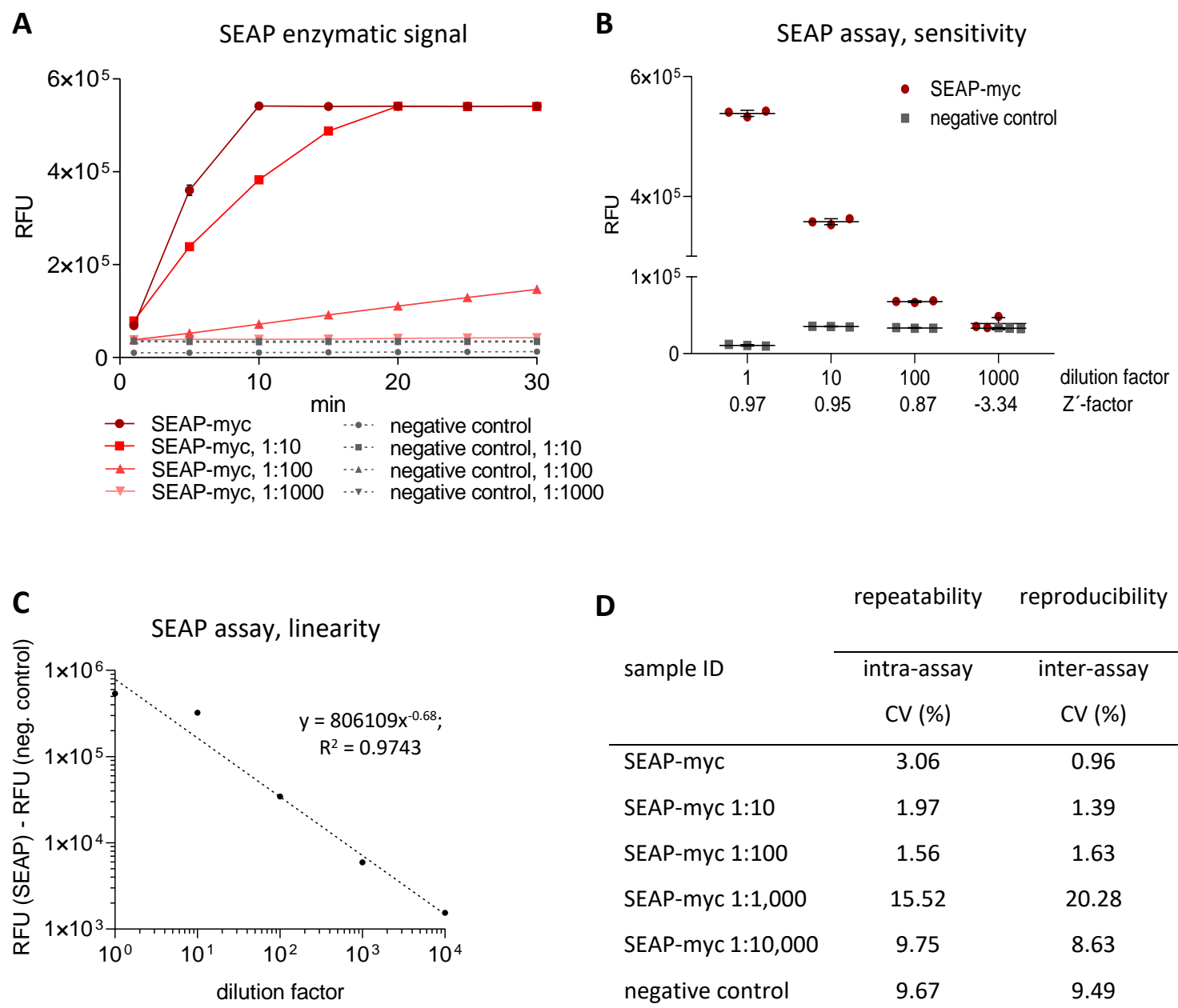

\begin{tabular}{lcc} 
D & repeatability & reproducibility \\
\cline { 2 - 3 } sample ID & intra-assay & inter-assay \\
& CV (\%) & CV (\%) \\
\hline SEAP-myc & 3.06 & 0.96 \\
SEAP-myc 1:10 & 1.97 & 1.39 \\
SEAP-myc 1:100 & 1.56 & 1.63 \\
SEAP-myc 1:1,000 & 15.52 & 20.28 \\
SEAP-myc 1:10,000 & 9.75 & 8.63 \\
negative control & 9.67 & 9.49 \\
\hline
\end{tabular}

Figure 8-4: Characterization of the fluorescent SEAP assay. SEAP-myc enzymatic activity was measured in undiluted and up to 1,000-fold diluted cell culture supernatants of corresponding HEK293T reporter cells, $24 \mathrm{~h}$ after cell seeding. Cell culture supernatant of HEK293T cells stably transfected with backbone were utilized as negative control. A: Kinetics of enzymatic reaction. SEAP enzymatic signal was measured for each of the dilutions for 30 minutes after substrate addition. Each point represents average signal \pm SD of three independent experiments. B: Determination of assay sensitivity. The highest dilution factor at which $Z^{\prime}$-factor is $\geq 0.5$ is 100 . Data represent mean \pm SD of three independent experiments. C: Determination of assay linearity. Colorimetric SEAP assay was linear $\left(R^{2}=0.9743\right)$ over four 10 -fold dilutions. Each point represents average signal of three independent experiments, detected five minutes after substrate addition. D: Determination of assay repeatability and reproducibility. The intra-assay and interassay CVs were determined between the three technical replicates or the three independent experiments, respectively. RFU: relative fluorescence units. 


\subsubsection{Characterization of eGFP assay}

Besides GLuc, I evaluated eGFP according to its suitability to be used as a reporter gene for normalization (cf. chapter 3.2.1.1). In contrary to GLuc, eGFP can be detected via fluorescent microscopy and its quantification requires no substrate addition.

First, I defined optimal excitation and emission wavelengths for eGFP detection at 488 and $510 \mathrm{~nm}$, respectively (Fig.8-5A). Next, I measured auto-fluorescence level of wild type THP-1 cells and different cell culture media, to determine assay background signal. As the phenol red-free cell culture medium was over three times less auto-fluorescent in comparison to the usual phenol red-containing medium (Fig.8-5B), it was used during eGFP assay characterization. eGFP fluorescent signal was proportional to the cell number of eGFP-expressing THP-1 reporter cells (Fig.8-5C). Signal of 150,000 THP-1 reporter cells was reduced by approximately $30 \%$ compared to this of 100,000 cells, whose signal in turn was halved relative to this of 50,000 cells (Fig.8-5C). eGFP assay displayed low assay sensitivity, indicated by the Z'-factor of 0.13 for 13,380 reporter cells(Fig.8-5D), unacceptable for HTS conduction in a 384-well format. eGFP fluorescent assay was not linear ("hockey-stick-effect") for smaller cell numbers (Fig.8-5E) and displayed an insufficient assay repeat- and reproducibility (Fig.8-5F). Especially the read-to-read CV (14.76- $21.25 \%)$, measured between the three consecutive reads of the same plate with no additional manipulation of the cells or the cell culture medium between the reads, was surprisingly high. Consequently, eGFP was not suitable to be used as a gene reporter for normalization. 
A

eGFP, excitation and emission

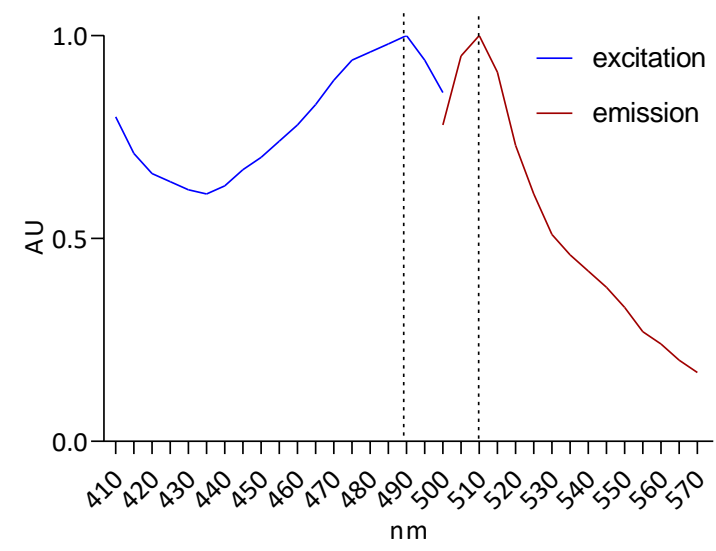

C

eGFP, signal strength

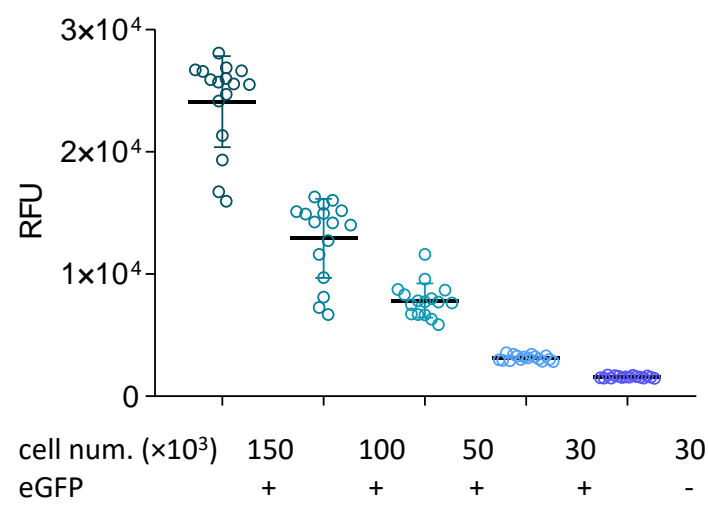

B

auto-fluorescence

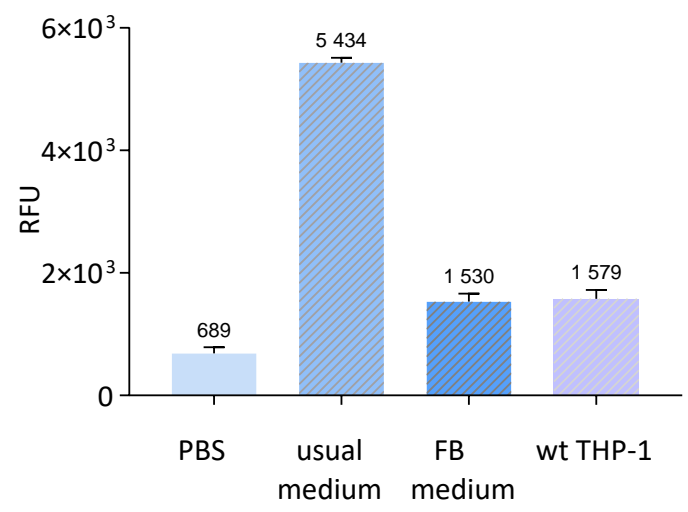

D

eGFP signal, sensitivity

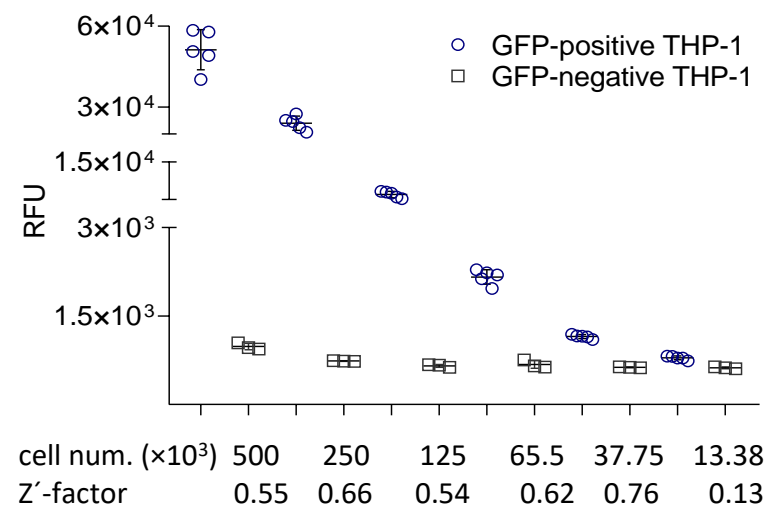

\section{E}

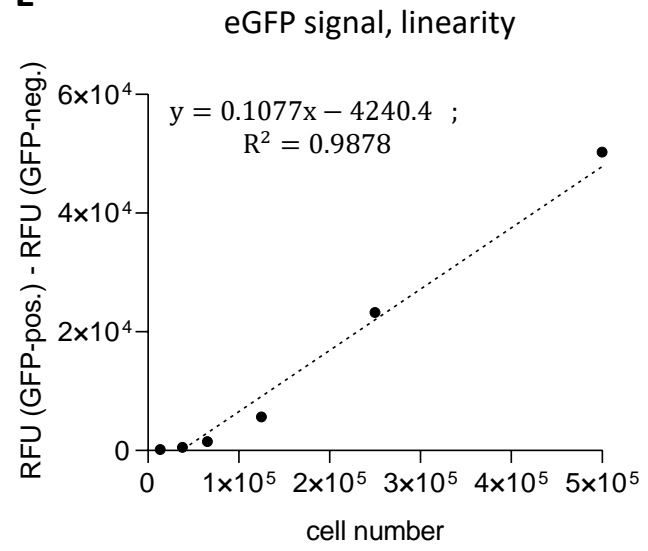

$\mathbf{F}$

repeatability

reproduci-

bility

\begin{tabular}{lccc}
\cline { 2 - 4 } cell number & $\begin{array}{c}\text { intra-assay } \\
\text { CV (\%) }\end{array}$ & $\begin{array}{c}\text { read-to-read } \\
\text { CV (\%) }\end{array}$ & $\begin{array}{c}\text { inter-assay } \\
\text { CV (\%) }\end{array}$ \\
\hline 150,000 & 17.13 & 18.50 & 56.05 \\
100,000 & 19.23 & 21.25 & 59.06 \\
50,000 & 12.60 & 19.47 & 42.34 \\
30,000 & 13.14 & 14.76 & 27.56 \\
neg. control & 8.04 & 7.35 & 5.85 \\
\hline
\end{tabular}

Figure 8-5: Characterization of eGFP assay. Fluorescent signal of eGFP-expressing THP-1 cells was measured in a 96-well format, 30 minutes after cell seeding. Wild type THP-1 cells were utilized as negative control. A: Determination of optimal excitation $(488 \mathrm{~nm})$ and emission $(510 \mathrm{~nm})$ wave lengths. Excitation and emission spectra were measured using eGFP-expressing THP-1 cells cultured in PBS. AU: arbitrary units B: Determination of auto-fluorescence of PBS, phenol-red containing cell culture medium (usual medium), FluoroBride ${ }^{\mathrm{TM}}$ phenol red-free cell culture medium (FB medium), and of 50,000 eGFP-negative wild type THP-1 cells in PBS. Each condition represents average signal \pm SD of three independent experiments. C: Determination of cell number-dependent eGFP signal strength. Data represent mean \pm SD of 16 individual wells. D: Determination of eGFP signal sensitivity. Fluorescent eGFP signals from equal numbers of serial diluted eGFP-positive and eGFP-negative cells (from 500,000 to 13,380) were measured. Data represent mean \pm SD of six individual wells. E: Determination of eGFP signal linearity for the in (D) measured cell numbers. Each point represents average signal \pm SD of six individual wells. F: Determination of the 
eGFP assay repeatability and reproducibly. The intra-assay, read-to-read, or inter-assay coefficients of variation were determined between the three technical replicates, the three consecutive reads of the same plate, or the three independent experiments, respectively.

\subsection{Characterization of CEBPD promoter strength}

During assay development, I generated two THP-1 reporter cell lines, which stably express one of the generated gene reporter constructs displaying following characteristics: i) SEAP expressed under control of the inducible CEBPD (CEBPD::SEAP) and GLuc - under control of the strong constitutive CMV (CMV::GLuc) promoters (multi-gene-reporter cassette 1.0); ii) SEAP expressed under control of CMV (CMV::SEAP) and GLuc - under control of CEBPD (CEBPD::GLuc) promoters (Fig.8-6A). For cloning strategies refer to chapter 8.8.3 Fig.8-37, Fig.8-38. To characterize CEBPD promoter strength in relation to this of CMV, I measured enzymatic activity of SEAP (Fig.8-6B) and GLuc (Fig. 8-6C) in cellular supernatants of corresponding PMA-differentiated THP-1 reporter $\mathrm{M} \varphi$. Enzymatic activity of CEBPD::SEAP was over 400-times lower in comparison to this of CMV::SEAP (Fig.8-6D). Also GLuc displayed lower enzymatic activity, when expressed under control of the defined CEBPD promoter in comparison to this when expressed under control of CMV (Fig.8-6E). Consequently, defined CEBPD promoter displays relatively weak promoter strength compared to this of CMV. 
A

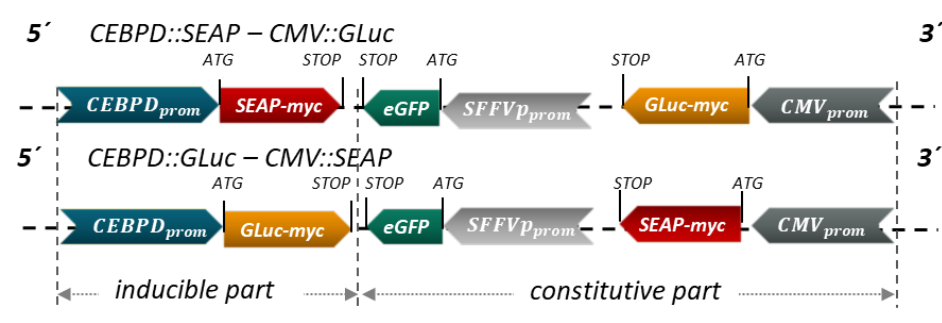

B

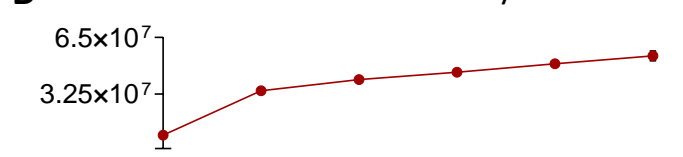

고
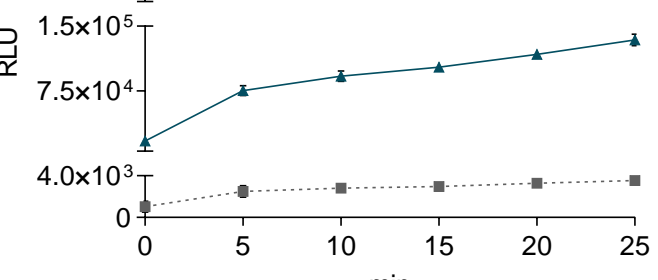

$\min$

$\rightarrow$ CMV::SEAP-myc $₫$ C/EBPD::SEAP-myc

-.. negative control

D

25 min time point

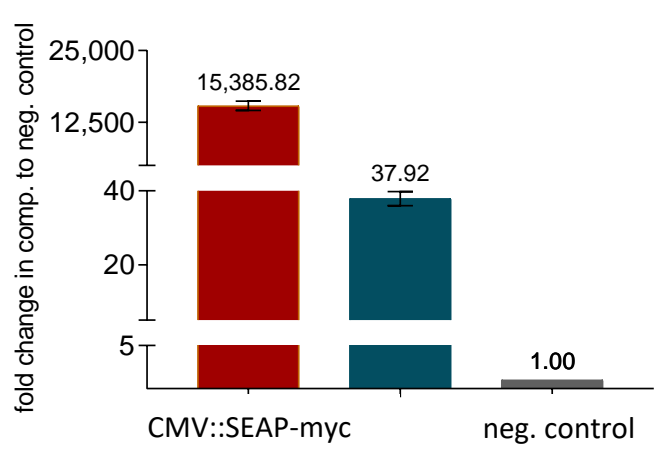

CEBPD::SEAP-myc
C

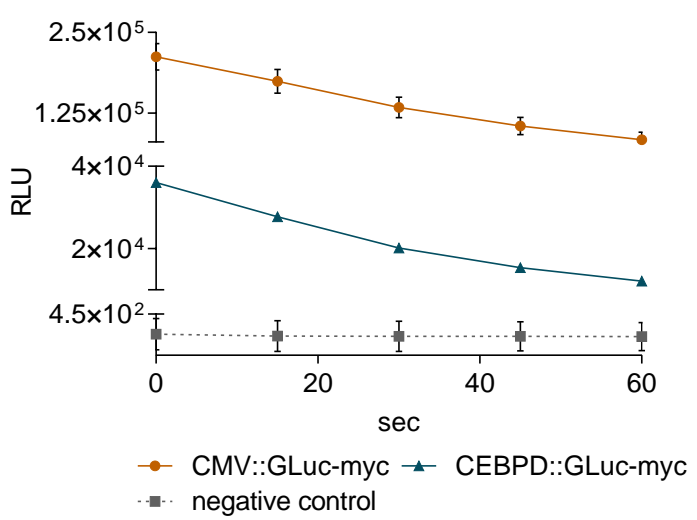

E

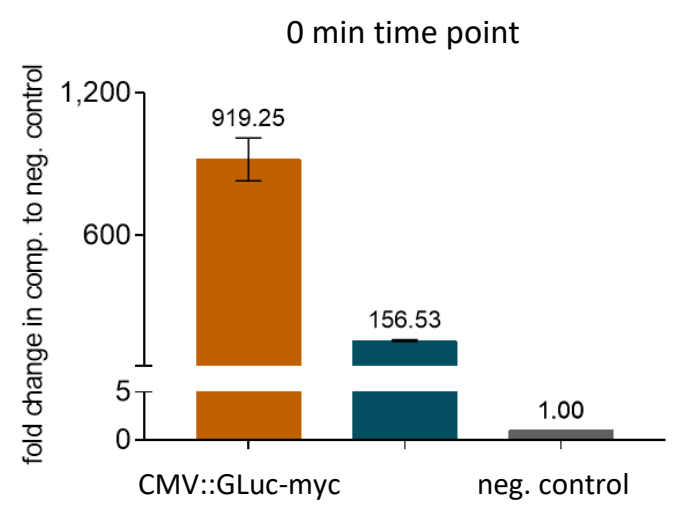

CEBPD::GLuc-myc

Figure 8-6: Characterization of CEBPD promoter strength. Two THP-1 reporter cell lines were generated by viral transduction of wild type THP-1 cells with lentiviral vectors encoding one of the generated gene reporter constructs (A). THP-1 wild type and reporter cells were differentiated by treatment with 50 $\mathrm{ng} / \mathrm{mL}$ PMA for 48 hours, in a 6-well format. Enzymatic activity of SEAP (B) and GLuc (C) was detected in cellular supernatants of THP-1 wild type and reporter $M \varphi, 72$ hours after PMA treatment. A: Schematic drawing of generated gene reporter constructs. B, C: Chemiluminescent SEAP and GLuc assays. Enzymatic activity of SEAP (B) and GLuC (C) were measured in cellular supernatants of THP-1 wild type (negative control) and reporter $M \varphi$ for 25 minutes and for 60 seconds after substrate addition, respectively. Data represent mean \pm SD of three independent experiments. C, D: Fold-changes in promoter-dependent SEAP (D) or Gaussia (E) enzymatic activities, shown relative to the negative control that was set as 1, demonstrated differences in relative promoter strength of CEBPD and CMV. Data represent mean \pm SD of three independent experiments. 


\subsection{Development of the final screening protocol}

\subsubsection{Cell culture condition in 384-well format}

SEAP signal intensity was an important factor for establishment of optimal cell culture condition in 384-well format. SEAP signal intensity depends on following factors: i) polarization; ii) cell culture duration; iii) differentiation format; iv) cell number. However, cell culture of THP-1 reporter cells in 384-well format also bears limitations and specialties such as cell number per well and differentiation format. Thus, optimal cell culture protocol was a summary of actions that results in the highest SEAP signal possible but considers the format- and cell-specific restrictions.

The ongoing methylation of GC-reach CEBPD promoter, which belongs to a huge CpGisland, can silence its activity leading to reduced SEAP secretion. Thus, I tested SEAP secretion level produced by non- and M1-polarized THP-1 reporter $\mathrm{M} \varphi$ that were cultured for one or five weeks after their generation. During HTS in 384-well format, the standard differentiation protocol of THP-1 reporter cells directly on the plate may negatively impact screening assay reproducibility (cf. chapter 3.2.3.2). Thus, I tested the effect of the alternative differentiation in a bulk format on SEAP secretion.

As expected, SEAP secretion level was generally higher in cellular supernatants of M1polarized (M1 condition) THP-1 reporter M $\varphi$ in comparison to this of non-polarized (M0 condition) cells, independent of cell culture duration and differentiation format (Fig.8-7A). THP-1 reporter $M \varphi$ displayed an equal over four-time fold induction in SEAP secretion level in response to $M 1$ treatment, independently from cell culture duration and cell number (Fig.8-7B). The 10,000 of M1-polarized THP-1 reporter M $\varphi$ (Fig.8-7A, blue dashed bar) produced a twice higher SEAP signal in comparison to this of 5,000 cells (Fig.8-7A, white bar). Although, 15,000 of THP-1 reporter cells (Fig.8-7A, light blue pointed bar) produced an approximately $30 \%$ higher SEAP signal in comparison to this of 10,000 cells (Fig.8-7A, light blue bar), it was not an appropriate cell number per well, as cells overgrew next day after seed.

SEAP secretion was approximately three times higher in cellular supernatants of 10,000 THP-1 reporter cells, which were differentiated directly on the plate (Fig.8-7A, blue dashed bar) relative to this when differentiated in a bulk format (Fig.8-7A, light blue bar). 
However, in bulk differentiation contributes to an equal differentiation state of THP-1 reporter cells and, thus, may improve assay repeat- and reproducibility. Differentiated in a bulk format, the 10,000 of M1-polarized one-week THP-1 reporter M $\varphi$ (Fig.8-7A, pink dashed bar) displayed a twice higher SEAP secretion level relative to this of 10,000 M1-polarized five-week THP-1 reporter M $\varphi$ (Fig.8-7A, light blue bar). During screening, therefore, it was important to use fresh aliquots of THP-1 reporter cells to avoid possible CEBPD promoter methylation-caused reduction in SEAP secretion.

A

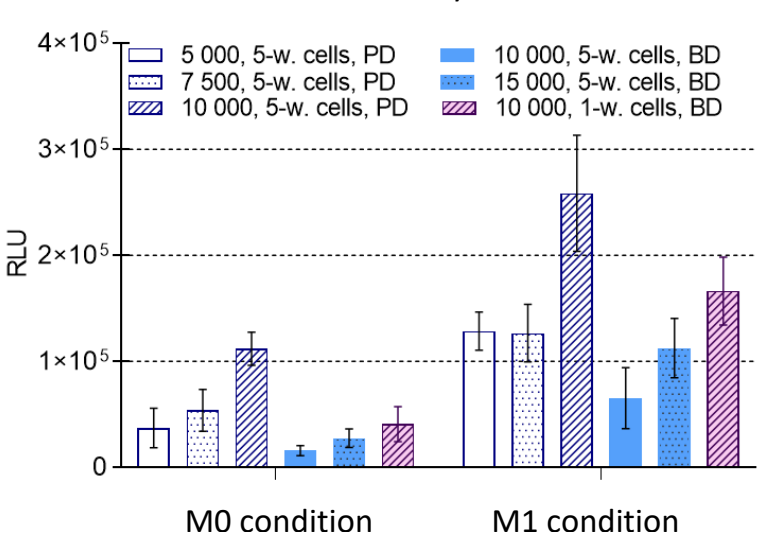

B

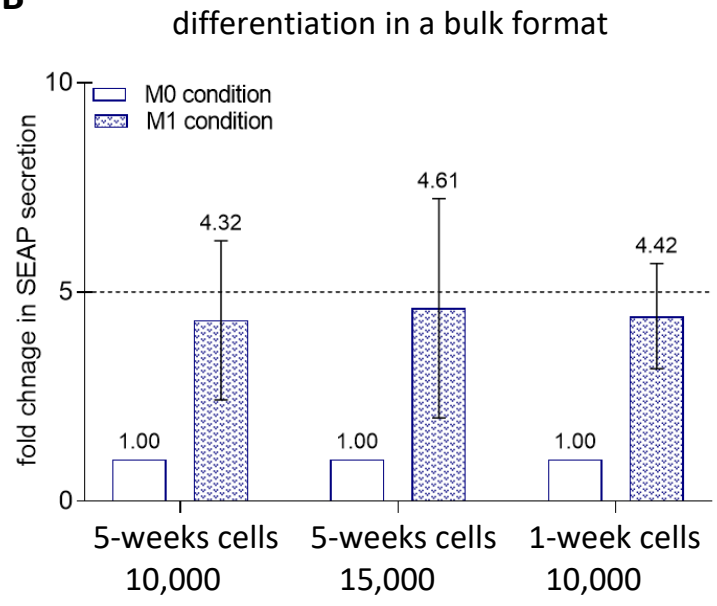

Figure 8-7: Establishment of the optimal cell culture condition in 384-well format. A: Chemiluminescent SEAP assay. THP-1 reporter cells, cultured for one (1-w. cells) or five weeks (5-w. cells) after generation, were PMA-differentiated in following formats: i) directly on a 384-well plate (PD) or ii) in a bulk format (BD). Corresponding SEAP secretion was analyzed in cellular supernatants of 5,000 to 15,000 of non- (M0 condition) or M1-polarized (M1 condition; treatment with $0.1 \mu \mathrm{g} / \mathrm{mL}$ LPS $+20 \mathrm{ng} / \mathrm{mL}$ IFN-g for $24 \mathrm{~h}$ ) THP-1 reporter $M \varphi$. Data represent mean \pm SD of three independent experiments. B: Fold-change in SEAP secretion level in response to the M1 treatment of in bulk format differentiated one- and five-week THP-1 reporter $M \varphi$, normalized to the corresponding $M 0$ condition. 


\subsubsection{Down-scaling and characterization of SEAP assay in 384-well format}

Down-scaling of SEAP readout assay from 96- to a 384-well format reduced HTS-dependent costs by factor five. I down-scaled SEAP assay by reduction of assay and sample volumes (data for different assay volumes are not shown). In a total volume of $30 \mu \mathrm{L}$, I characterized SEAP signal stability and sensitivity using cellular supernatants of M1-polarized THP-1 reporter $\mathrm{M} \varphi$ that express multi-gene-reporter-cassette 1.0 under control of CEBPD target promoter (Fig.8-37). Detected for small sample volumes (5-1.25 $\mu \mathrm{L}$ ), SEAP reporter displayed the highest signal stability for sample volume of $5 \mu \mathrm{L}$ (Fig.8-8A). Chemiluminescent SEAP assay was also very sensitive, due to an optimal $Z^{\prime}$-factor $\geq 0.5$ for all sample volumes tested (Fig.8-8B).

A

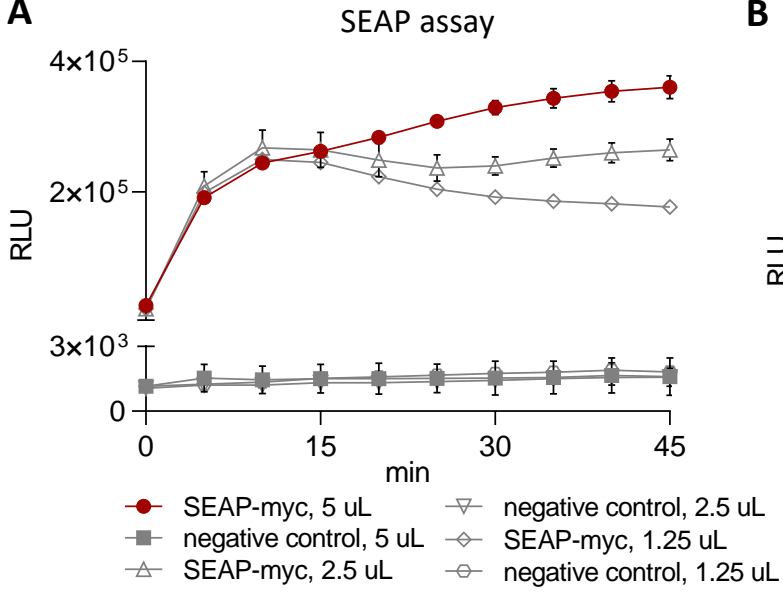

B

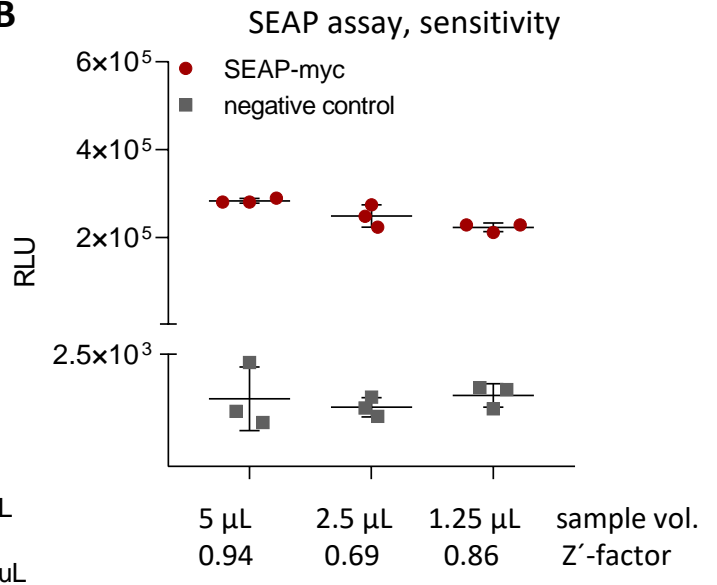

Figure 8-8: Characterization of the chemiluminescent SEAP assay for small sample volumes. PMA-differentiated THP-1 reporter $M \varphi$ were M1-polarized by treatment with $0.1 \mu \mathrm{g} / \mathrm{mL} \mathrm{LPS}+20 \mathrm{ng} / \mathrm{mL}$ IFN-g. SEAP enzymatic activity was measured in cellular supernatants of THP-1 reporter $M \varphi, 24 \mathrm{~h}$ post-treatment. Negative control was represented by cellular supernatant of non-polarized wild type THP-1 M $\varphi$. Data represent mean \pm SD of three independent experiments. A: Chemiluminescent SEAP assay. Sample volumedependent ( 5 - $1.25 \mu \mathrm{L}$ ) SEAP signal was monitored for 45 minutes after substrate addition. B: Determination of assay sensitivity. 


\subsection{LOPAC $^{\circledR 1280}$ and ENZO ${ }^{\circledR 774}$ compound libraries}

LOPAC (list of pharmacological active compounds) library contains 1280 experimentally validated small molecules that display versatile chemical and pharmacological properties (Fig.8-9). Commercially available LOPAC library ${ }^{11 *}$ is used during differently purposed high-throughput screenings. ${ }^{329-331} \mathrm{ENZO}^{\circledR 774}$ compound library contains 774 FDA approved drug compounds, which belong to various indication classes (Fig.8-10) and display known bioactivity, safety, and bioavailability. ${ }^{12}$ Thus, ENZO ${ }^{\circledR 774}$ library is used not only for identification of signalling mediators ${ }^{332,333}$ but also for drug repurposing. ${ }^{334}$

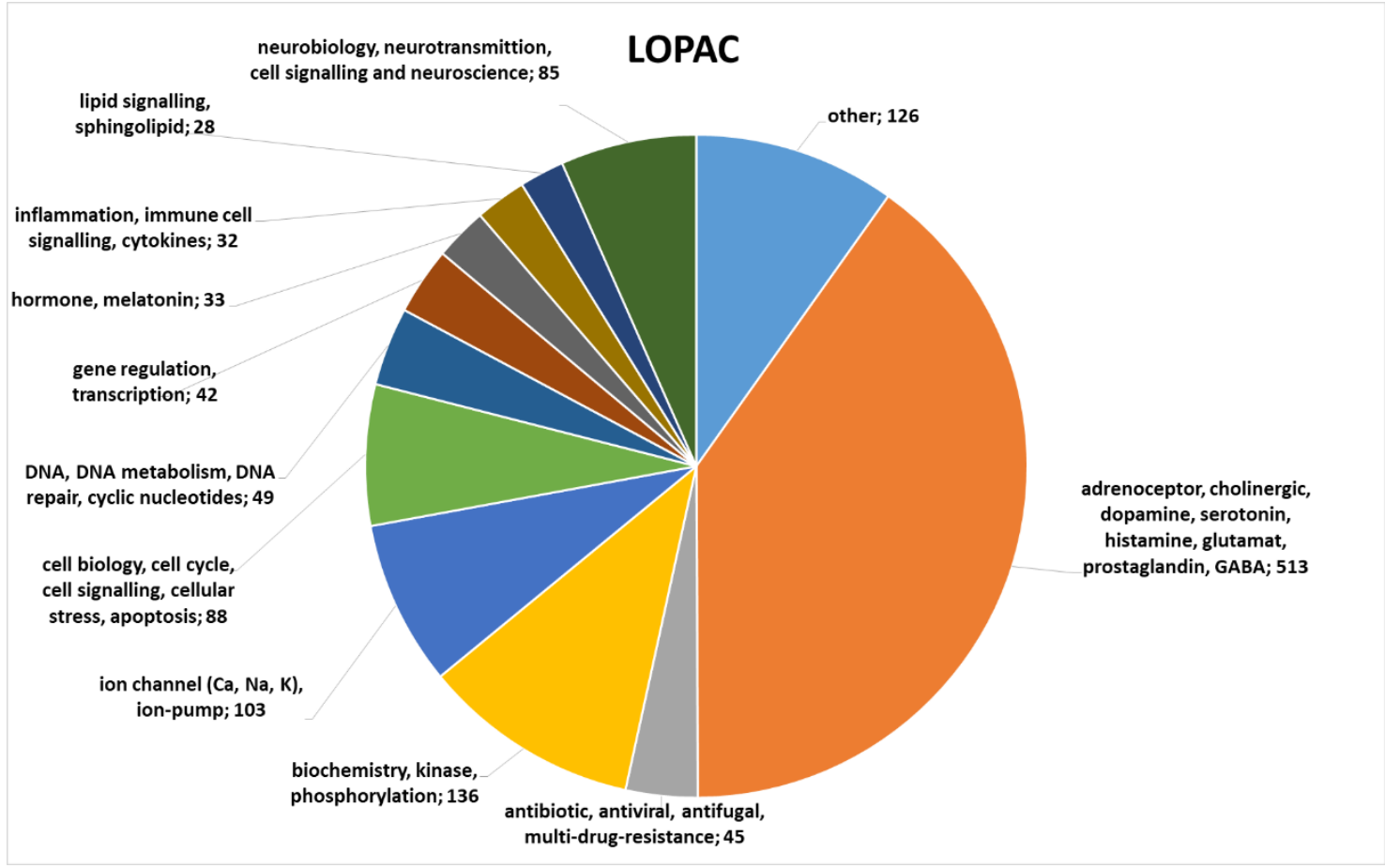

Figure 8-9: Pharmacological classes of compounds from LOPAC ${ }^{\circledR 1280}$ library. LOPAC ${ }^{\circledR}$ contains 1280 compounds that display various pharmacological properties summarized here to distinct classes. Digits indicate the number of compounds in each class. In this figure I analysed a raw library information, which was kindly provided by colleagues from Fraunhofer IME ScreeningPort in Hamburg.

The nein selected compounds from LOPAC ${ }^{\circledR 1280}$ library display their pharmacological activity in following classes: gene regulation and transcription (GSK 1210151A and

$11 *$ LOPAC $^{\oplus 1280}$ - The Library of Pharmacologically Active Compounds. Merck KGaA website. https://www.sigmaaldrich.com/life-science/cell-biology/bioactive-small-molecules/lopac1280-navigator.html. Accessed February 20, 2020.

$12 * *$ SCREEN-WELL ${ }^{\circledR}$ FDA approved drug library V2. Enzo Life Sciences, Inc. website.

https://www.enzolifesciences.com/BML-2843/screen-well-fda-approved-drug-library-v2/. Accessed February $20,2020$. 
13-cis-retinoic acid), lipid signalling (Ro 11-1464), neurobiology and -transmission (Ro 61-8048), cellular stress (danshensu sodium salt), immunomodulation (bropirimine), estrogen receptor targeting (lasofoxifene tartrate), ion pump targeting (sanguinarine chloride), and enzymatic phosphorylation (roscovitine).

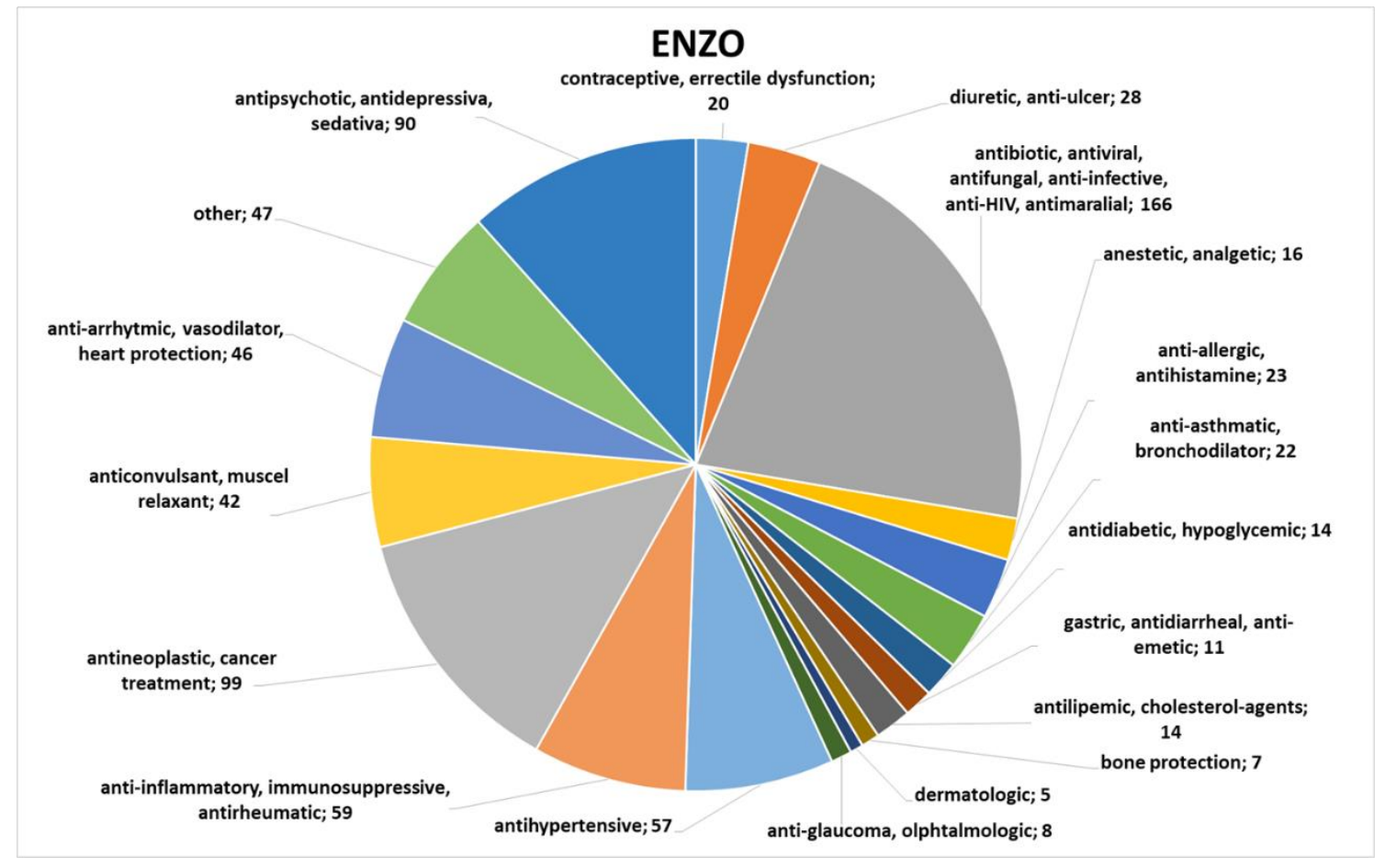

Figure 8-10: Therapeutic classes of compounds from ENZO ${ }^{\circledR 774}$ library. ENZO ${ }^{\circledR}$ contains 774 approved drug compounds that are summarized here according to their indication. Digits indicate the number of compounds in each class. In this figure I analysed a raw library information, which was kindly provided by colleagues from Fraunhofer IME ScreeningPort in Hamburg.

According to their therapeutic characteristics, the nine selected compounds from ENZO ${ }^{\circledR 74}$ library act as follows: antineoplastic (vorinostat, auranofin, and mitoxantrone), anti-inflammatory (leflunomide (DMARD), ketorolac tromethamine (NSAID), fluocinonide, amcinonide, and halcinonide), and anti-asthmatic (zileuton). 


\subsection{Screening for CEBPD-modulating compounds}

\subsubsection{High-throughput screening}

A

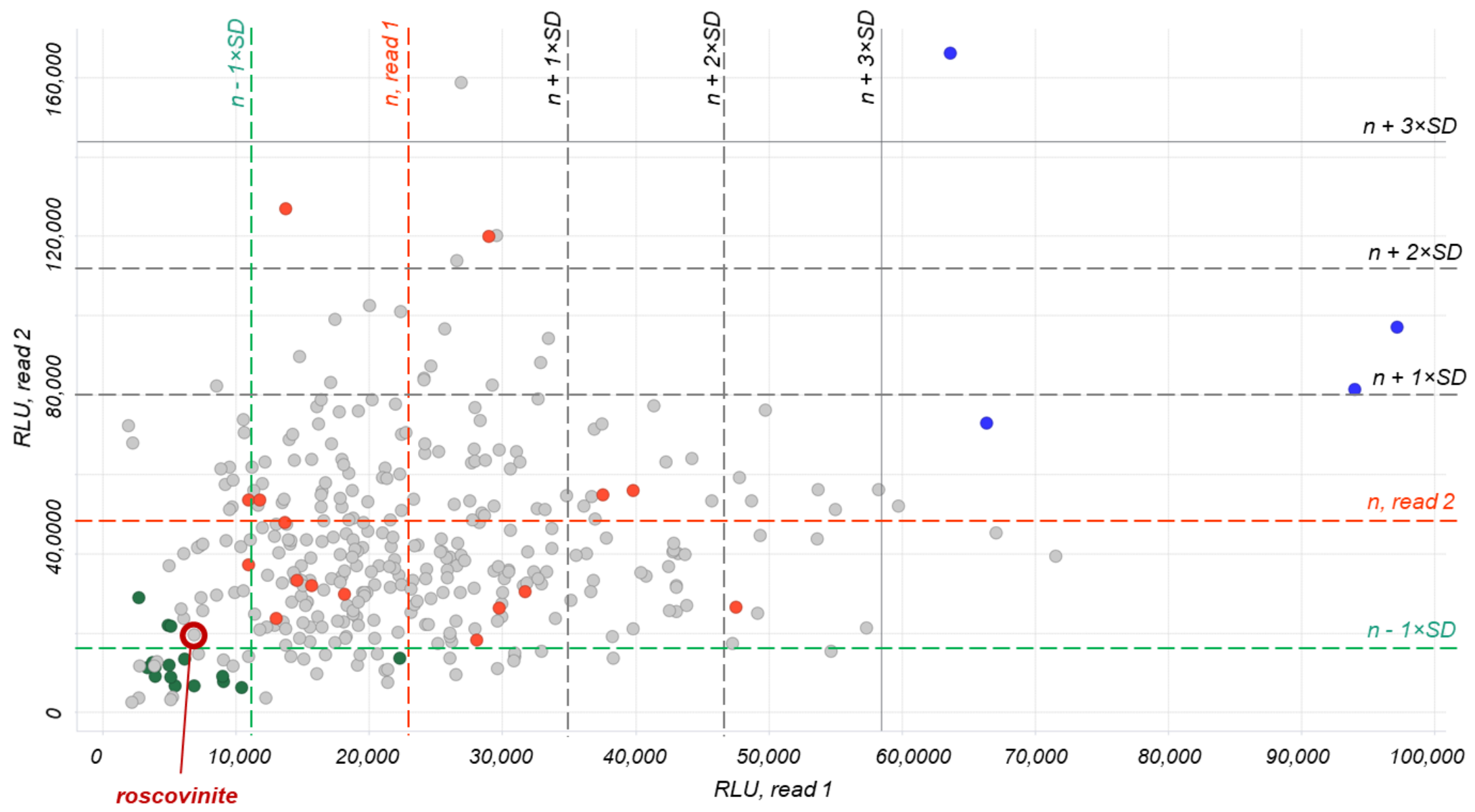

Figure 8-11: Identification of hit compounds derived from screening of LOPAC ${ }^{\oplus 1280}$ library. Figure continued on the next page. 
Figure 8-9: Continuance.

B

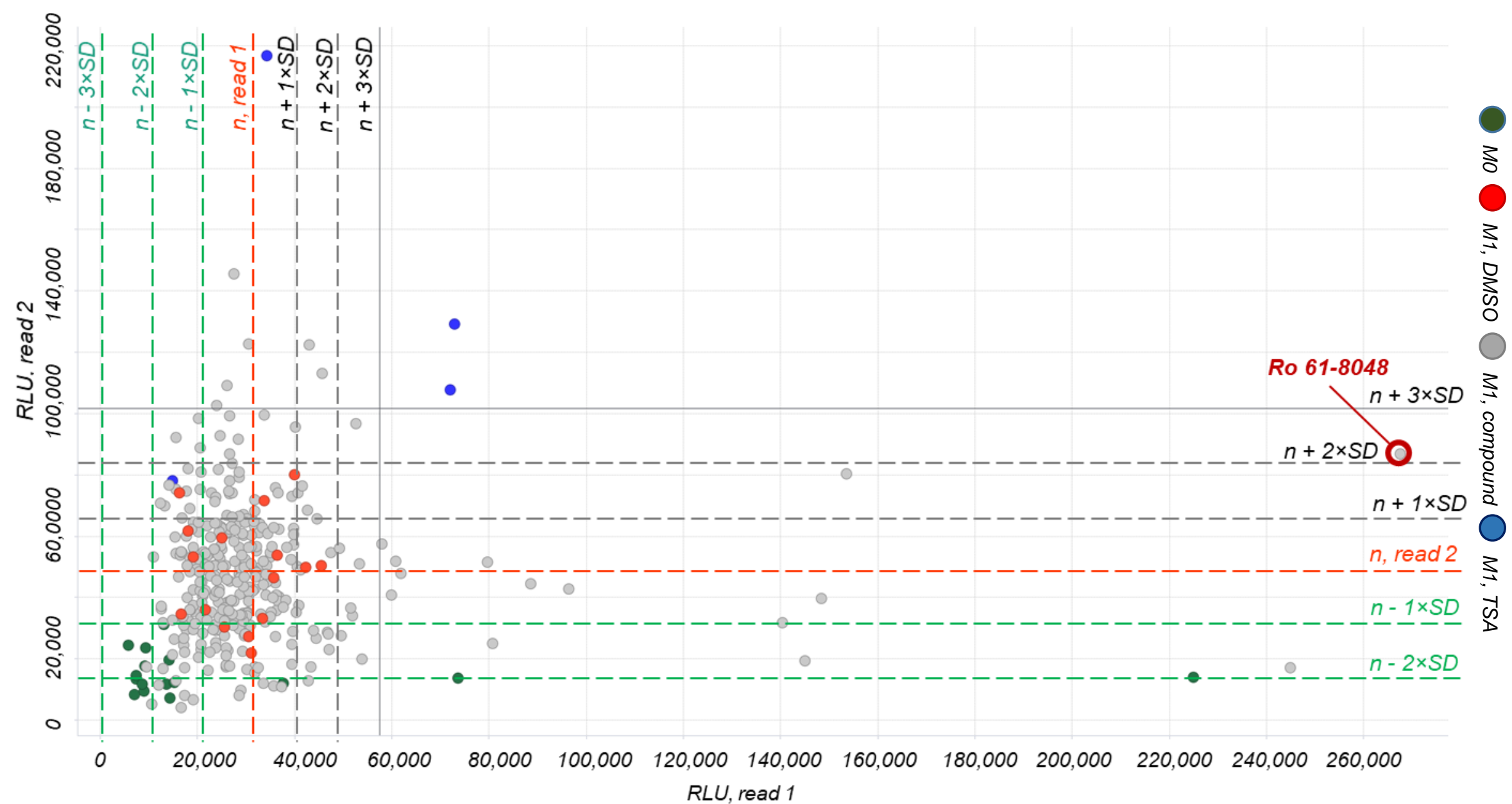

Figure 8-11: Identification of hit compounds derived from screening of LOPAC ${ }^{\circledR 1280}$ library.

Figure continued on the next page. 
C

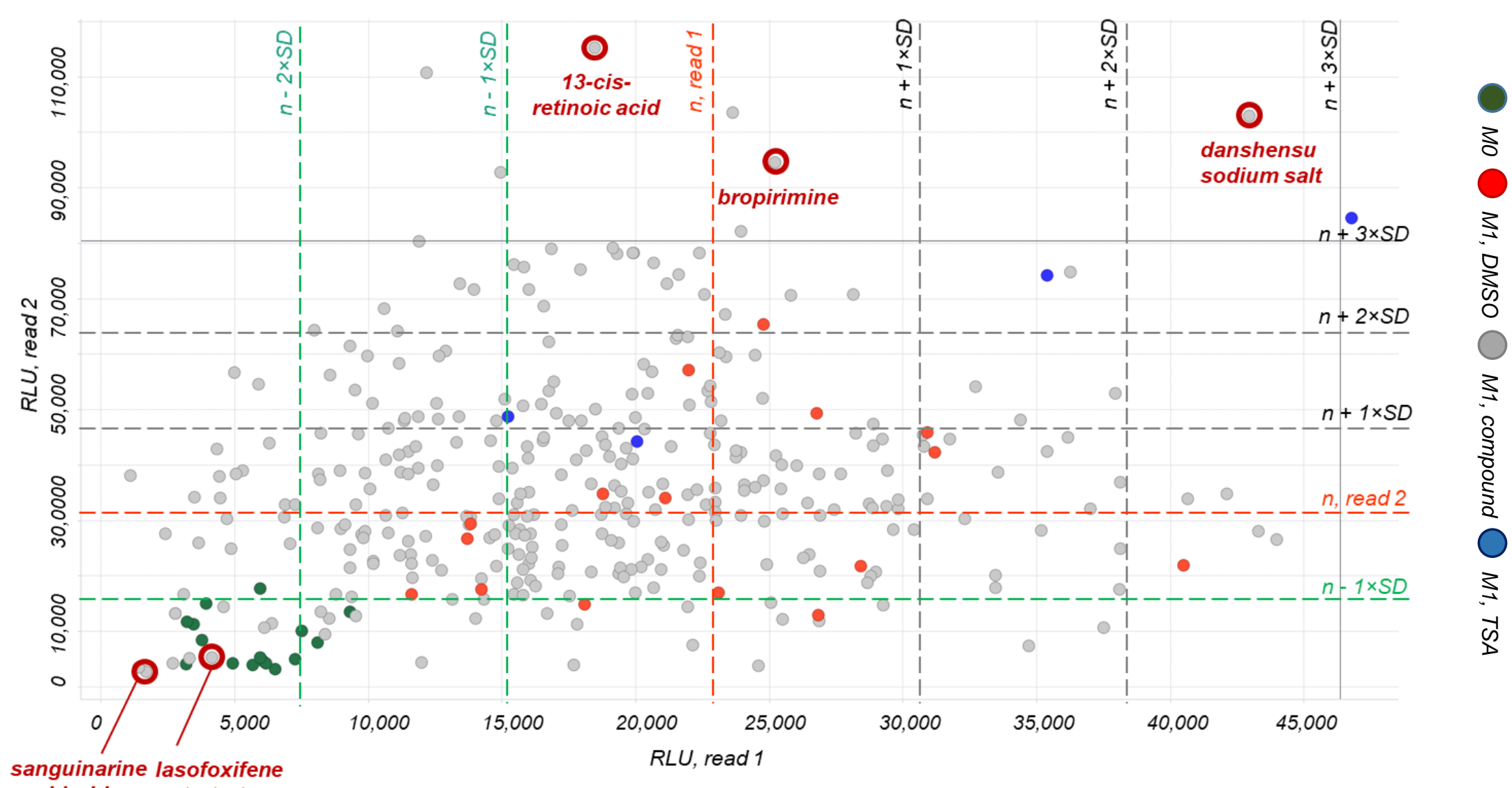

chloride

Figure 8-11: Identification of hit compounds derived from screening of LOPAC ${ }^{\otimes 1280}$ library. A, B, C: Screening of LOPAC ${ }^{\oplus 1280}$ library (1280 compounds) included four 384 -well plates, three of which are shown here (A, B, C). Enzymatic SEAP signals were detected in cellular supernatants of non-polarized and non-treated (MO control; 16 wells) and M1-polarized either with DMSO (M1, DMSO; 16 wells), compound (M1, compound; up to 336 wells), or TSA (M1, TSA; 4 wells) pre-treated THP-1 reporter M $\varphi$. As primary screening was performed twice, SEAP signals from read 1 are plotted versus corresponding signals from read 2 . Compounds showing strong activatory or inhibitory effect on SEAP secretion were identified in areas over ( $n+1 / 2 / 3 \times S D$, black line) or under ( $n-1 / 2 \times S D$, green line) up to three SDs of mean, calculated for "M1, DMSO" (solvent control) condition ( $n$, read line), respectively. Selected compounds (red circles) were summarized in Table 3.3 (chapter 3.3.1). 
A

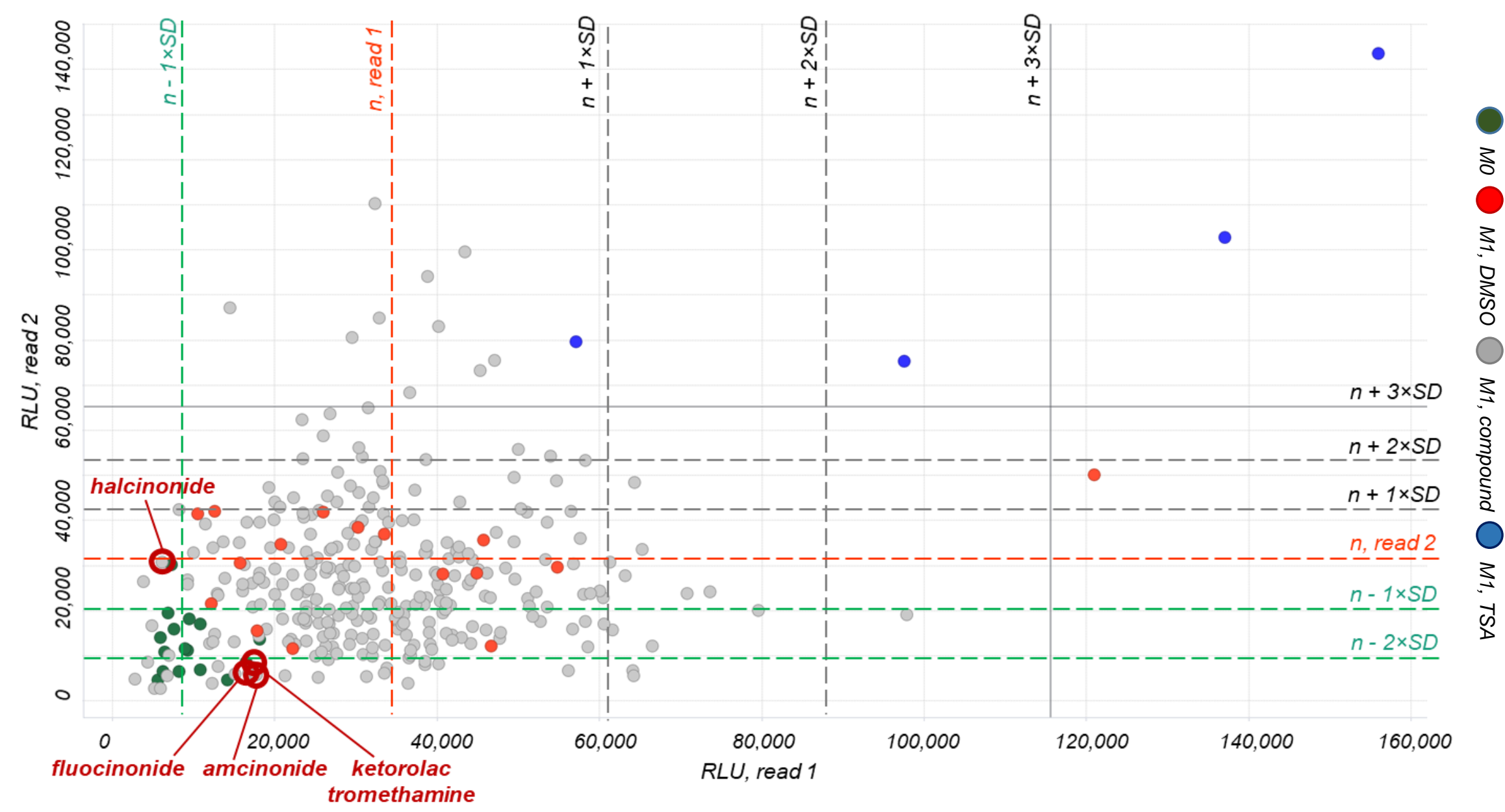

Figure 8-12: Identification of hit compounds derived from screening of ENZO ${ }^{\otimes 774}$ library.

Figure continued on the next page. 
B

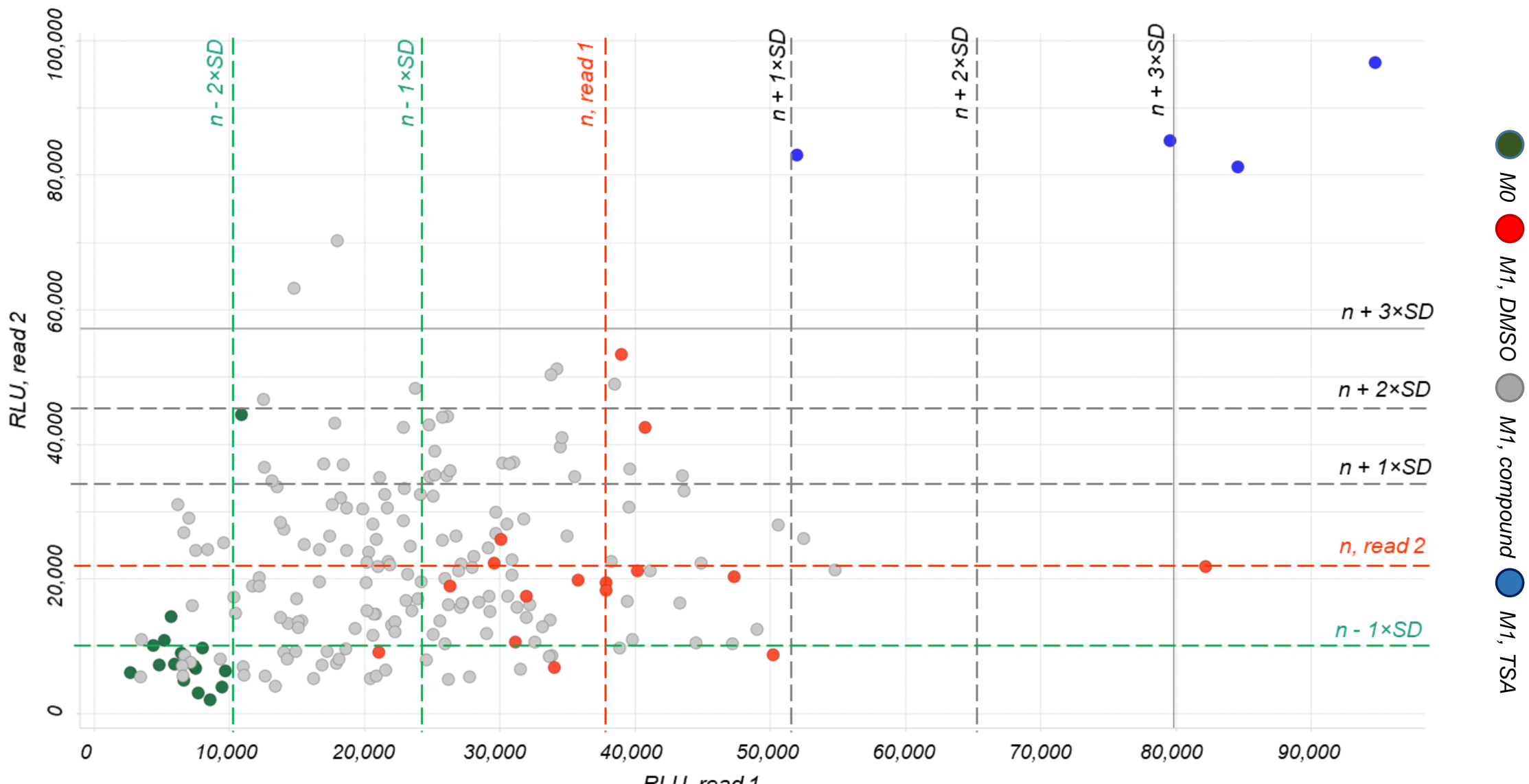

Figure 8-12: Identification of hit compounds derived from screening of ENZO ${ }^{\oplus 744}$ library. Screening of ENZO ${ }^{\circledR 774}$ library (774 compounds) included three 384 -well plates, two of which are shown here (A, B). Enzymatic SEAP signals were detected in cellular supernatants of non-polarized and non-treated (M0 control; 16 wells) and M1-polarized either with DMSO (M1, DMSO; 16 wells), compound (M1, compound; up to 336 wells), or TSA (M1, TSA; 4 wells) pre-treated THP-1 reporter M $\varphi$. As primary screening was performed twice, SEAP signals from read 1 are plotted versus corresponding signals from read 2. Compounds showing strong activatory or inhibitory effect on SEAP secretion level were identified in areas over $(n+1 / 2 / 3 \times S D$, black line) or under ( $n-1 / 2 \times S D$, green line) up to three SDs of mean, calculated for "M1, DMSO" (solvent control) condition ( $n$, read line), respectively. Selected compounds (red circles) were summarized in Table 3.3 (chapter 3.3.1). 


\subsubsection{Hit compound confirmation}

During HTS for CEBPD-modulating compounds, 18 compounds were selected: nine with the highest and nine with the lowest SEAP signals (chapter 3.3.1, Table 3.3). To confirm the observed compound-mediated effects, THP-1 reporter cells were treated with selected compounds in a 96-well format according to the developed screening protocol (chapter 2.39.2). Enzymatic SEAP signals (Fig.8-11A, B) were normalized to a corresponding cell number values determined by CellTiter-Glo ${ }^{\circledR}$ assay (Fig.8-11C, D).

A
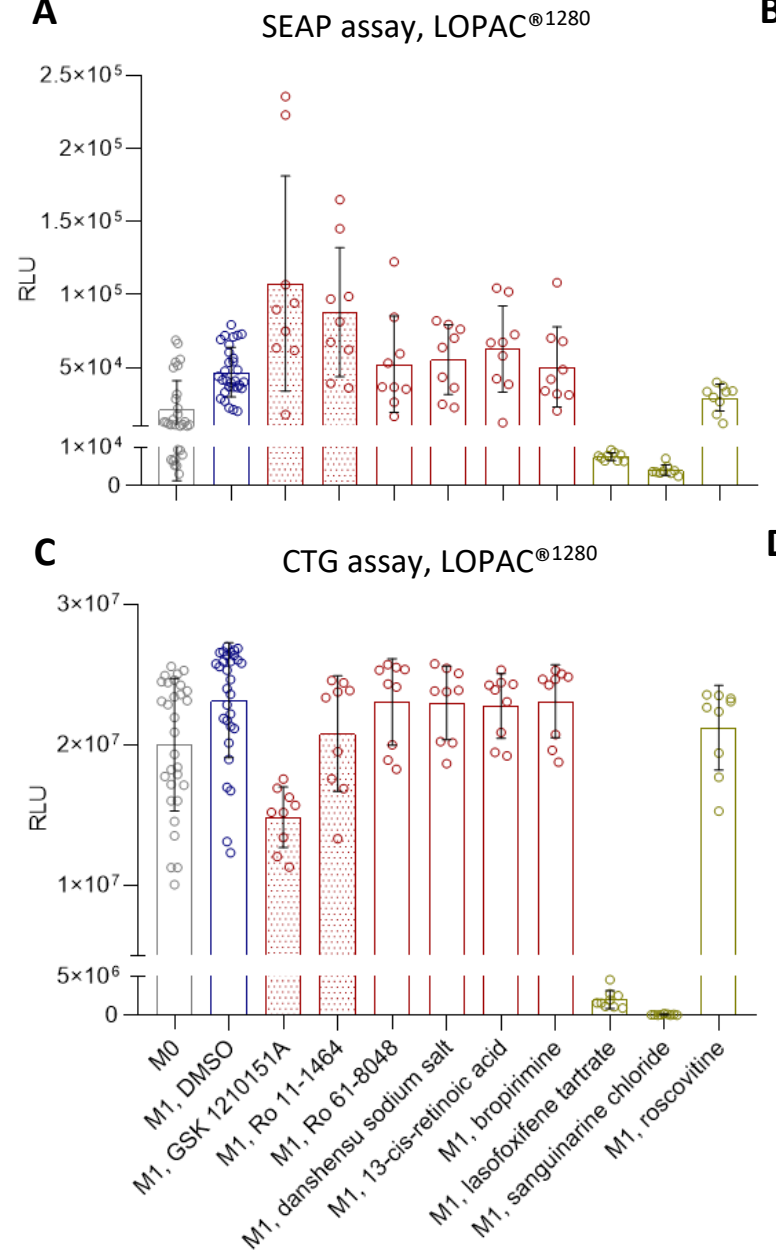

B

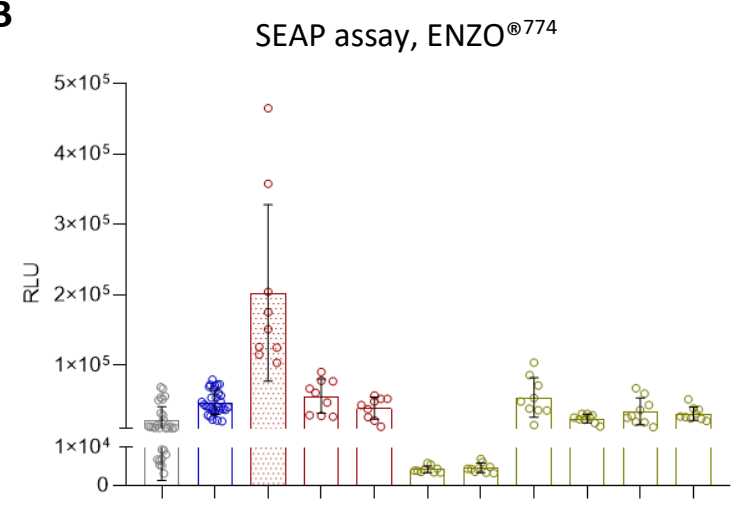

D

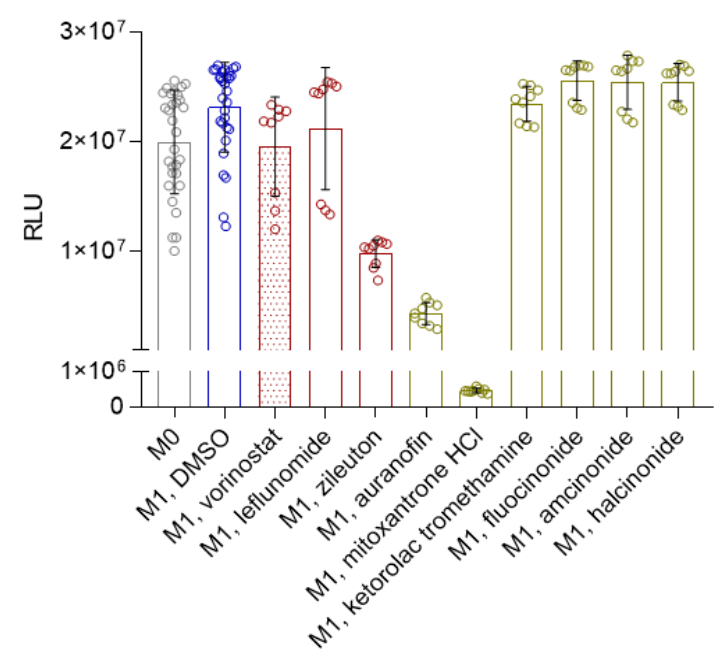

Figure 8-13: Conformational analysis of selected compounds from LOPAC ${ }^{\oplus 1280}$ and $\mathrm{ENZO}^{\circledR 774}$ libraries: THP-1 reporter cells were PMA-differentiated and treated with selected compounds from LOPAC $^{\otimes 1280}$ (A, C) and $\mathrm{ENZO}^{\otimes 774}(\mathrm{~B}, \mathrm{D})$ libraries in a 96-well format, as described previously (chapter 2.39.2). SEAP signals (A, B), detected in response to the pre-treatment with activatory- (red bars), A-ranked (red dotted bars), or inhibitory-acting (green bars) compounds, were normalized to corresponding cell number values detected by CellTiter-Glo ${ }^{\circledast}$ assay (CTG) (C, D). Non-polarized and non-treated THP-1 reporter M $\varphi$ represented $\mathrm{MO}$ control condition (grey bars), while M1-polarized and DMSO-treated THP-1 reporter $\mathrm{M} \varphi$ represented solvent control condition (blue bars). A, B: Chemiluminescent SEAP assay. LOPAC ${ }^{\oplus 1280}$ (A) and ENZO $^{\otimes 774}$ (B) compound-mediated changes in SEAP secretion level were determined in cellular supernatants of corresponding THP-1 reporter M $\varphi$. C, D: Chemiluminescent CTG assay. LOPAC ${ }^{\oplus 1280}$ (C) and $\mathrm{ENZO}^{\oplus 774}$ (D) compound-mediated changes in cell number were determined in lysates of corresponding THP-1 reporter $M \varphi$. Data represent mean \pm SD of 9 to 30 single wells per condition. 


\subsubsection{Signal correlation}

Eightteen compounds were selected during HTS for CEBPD-modulating compounds, which was performed in 384-well format twice (read 1 and read 2). Their effects on SEAP secretion were further evaluated by hit conformation and hit characterization, performed in 96- and 24-well formats, respectively. Correlation between corresponding SEAP signals (Fig.8-14) may indicate reproducibility and therefore reliability of the performed screening assay.
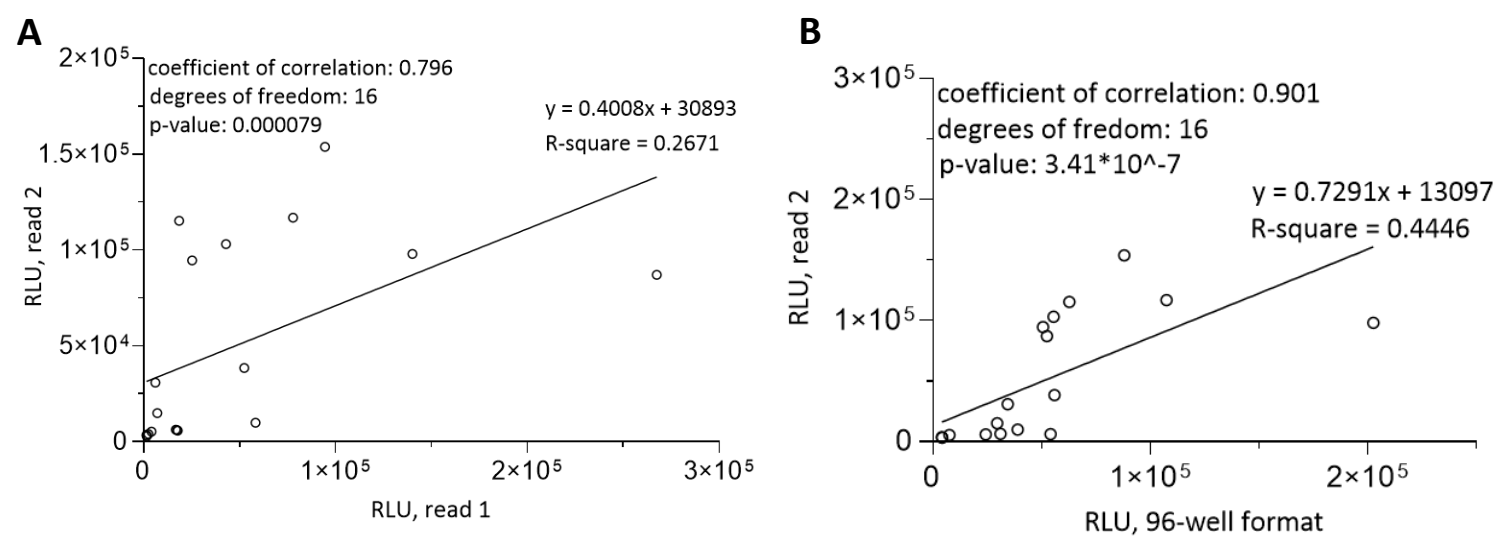

C

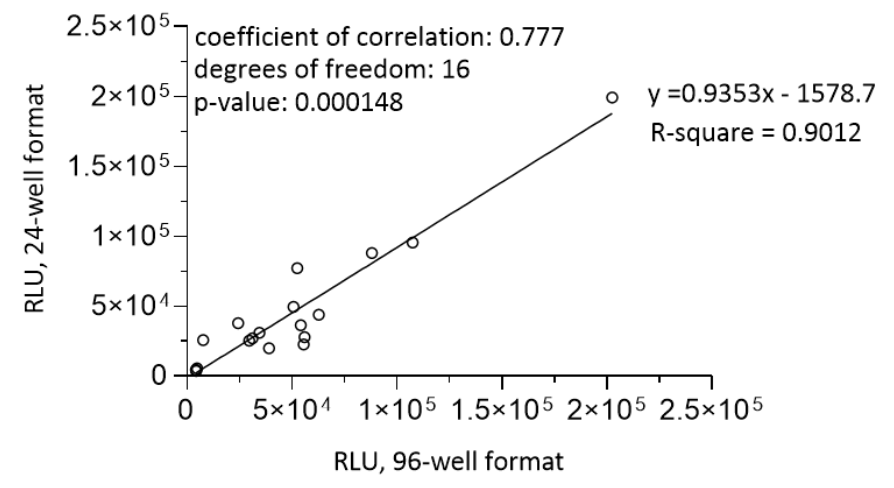

Figure 8-14: Correlation between compound-mediated SEAP readouts. A, B, C: Spearman rank correlation statistical analysis. Coefficient of correlation, degrees of freedom, $p$-values, as well as equation and $\mathrm{R}$-square of trend lines are displayed on individual graphs. A: Correlation of compound-caused SEAP enzymatic signals between read 1 and read 2 (HTS). B: Correlation of compound-caused SEAP enzymatic signals between read 2 (HTS) and 96-well format (hit confirmation). C: Correlation of compound-caused SEAP enzymatic signals between 96- (hit confirmation) and 24-well formats (hit characterization). 


\subsubsection{Hit compound characterization via RT-qPCR}

Gene expression analysis by real-time quantitative PCR in THP-1 reporter $M \varphi$ required selection of robust and reliable house-keeping genes. Ribosomal protein 37A (RPL37A) and $ß$-actin (ACTB) are reported to display the highest stability during differentiation of THP-1 M $\varphi .{ }^{210}$ In my experimental setting, following factors needed to be considered additionally: i) lack of introns in CEBPD gene and ii) presence of pseudo-genes in THP-1 reporter cells.

For an appropriate RT-qPCR analysis it was crucial that the tested sample is not contaminated with genomic DNA (gDNA) and the obtained RT-qPCR product originates definitely from cDNA. Potential gDNA contamination can be detected by a special primer design, when gene-specific forward and reverse primers locate in two different exons and flank at least one intron. However, such primer design is only possible for introncontaining genes like RPL37A (Fig.8-15A), which RT-qPCR product from cDNA is much smaller relative to this from gDNA (Fig.8-15D). For the intronless CEBPD gene (Fig.8-15B), the RT-qPCR products from cDNA and gDNA are of the same size (Fig.8-15E). Additionally, some house-keeping genes like ACTB exist as intronless pseudo-genes in the genome of THP-1 reporter cells. The RT-qPCR products from cDNA and gDNA of ACTB gene (Fig.8-15C) were also of the same size (Fig.8-15F). During RT-qPCR analysis of hit compound-mediated effects on gene expression in THP-1 reporter $\mathrm{M} \varphi$ ( $c f$. chapter 3.4), each RT-qPCR sample was tested according to the gDNA contamination using RPL37A primer pair.

RPL37A was also used as a house-keeping gene, but only for the samples, which were collected 4 hours after M1 treatment. Ct values of RPL37A were significantly higher relative to corresponding Ct values of M0 condition (Fig.8-15G), 24 hours after M1-treatment. Instead, ACTB was used as a house-keeping gene for samples, which were collected at the later time point, as ACTB Ct values were stable relative to the $\mathrm{MO}$ condition at both time points tested (Fig.8-15H). 


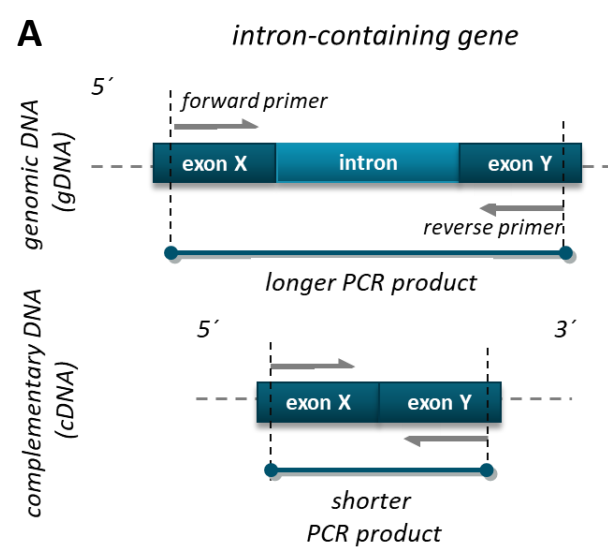

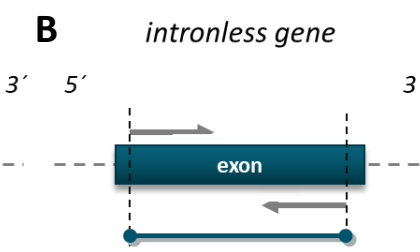

PCR product
C pseudo-gene
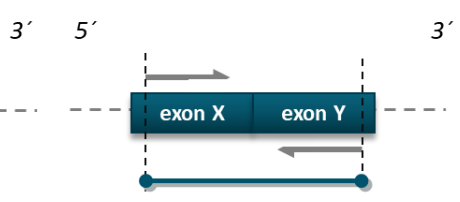

PCR product

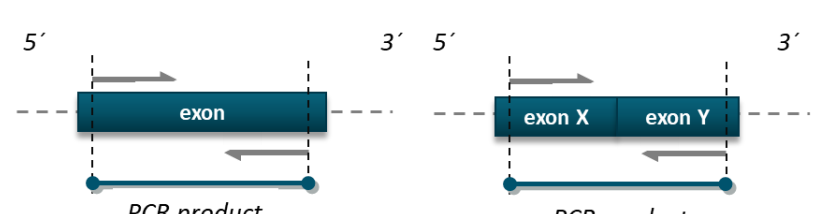

PCR product
D

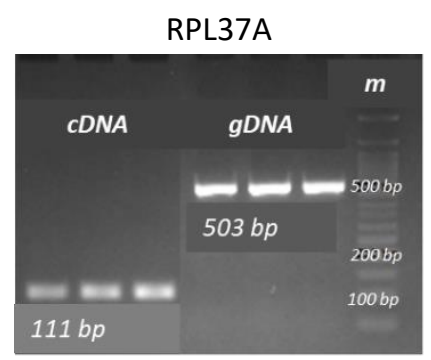

E

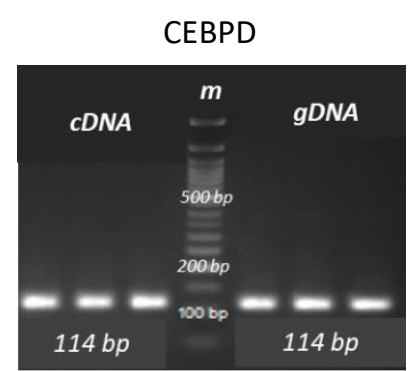

F

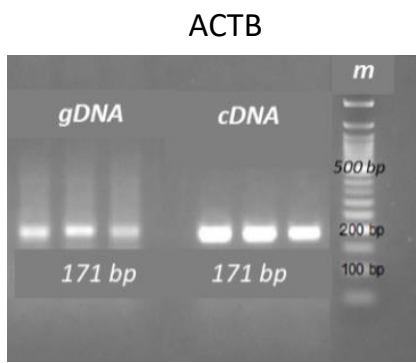

G

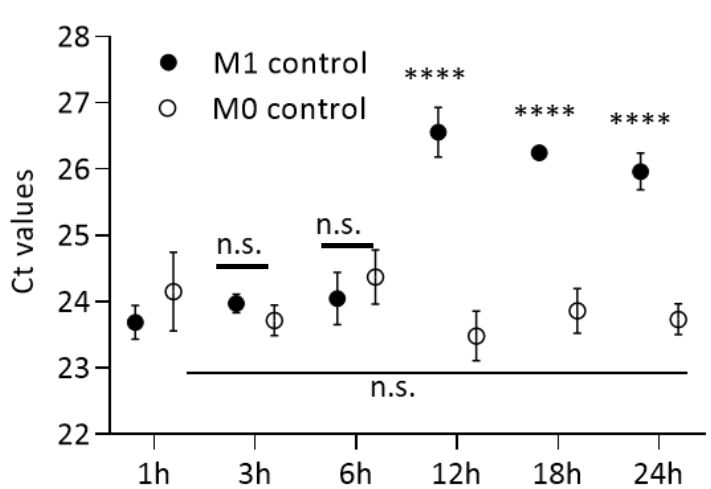

H
ACTB

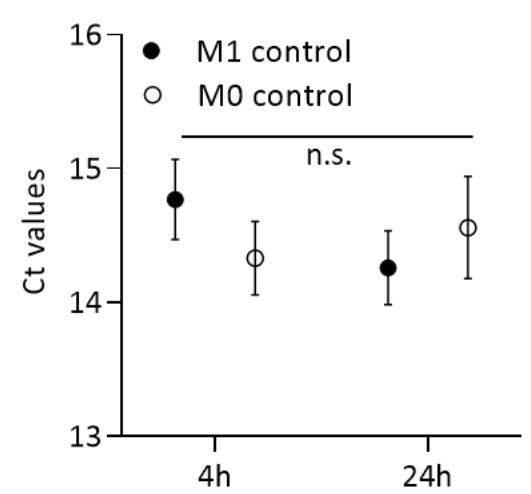

Figure 8-15: Selection of house-keeping genes for RT-qPCR. A, D: When forward and reverse primers locate in two different exons and flank at least one intron (A), the resulting RT-qPCR products from cDNA and gDNA differ in their size: a bigger product originates from gDNA and a smaller product - from cDNA, as shown for an intron-containing RPL37A gene (D). B, E: For intronless genes like CEBPD (B), the RT-qPCR products that originate from the gDNA or CDNA are of the same size (E). C, F: Also pseudo-genes, which are expressed in the genome in their intronless version (C), display equal-sized RT-qPCR products from gDNA and CDNA, as shown for ACTB (F). G, H: THP-1 reporter cells were PMA-differentiated and then M1-polarized by treatment with $0.1 \mu \mathrm{g} / \mathrm{mL}$ LPS $+20 \mathrm{ng} / \mathrm{mL}$ IFN-g for 24 hours. Ct values of RPL37A (G) and ACTB $(\mathrm{H})$ house-keeping genes were analysed in lysates of non-polarised and non-treated (M0 condition) or M1-polarized (M1 condition) THP-1 reporter $M \varphi$, at indicated time points after $M 1$ treatment. G: Timedependent differences of RPL37A Ct values in M1 condition were analysed via ordinary One Way ANOVA with Dunnett's correction for multiple comparisons. Time-dependent differences of RPL37A Ct values in MO condition were analysed via Kruskal-Wallis test with Dunnett's correction for multiple comparisons. Data represent mean \pm SD of six independent experiments. H: Time-dependent differences of ACTB Ct values in M1 and M0 conditions were analysed via Kruskal-Wallis test with Dunnett's correction for multiple comparisons. Data represent mean \pm SD of 20 independent experiments. ${ }^{* * * * p}<0.0001$; ns: not significant; m: 50 bp DNA marker; bp: base pairs. 


\subsection{Analysis of TSA- and vorinostat-mediated effects in a longitudinal study}

The observed TSA- and vorinostat-mediated effects on SEAP secretion and gene expression of endogenous CEBPD, reporter CEBPD::SEAP, and CCL2 (chapter 3.4, Fig.3-18), I further analyzed in a longitudinal study (Fig.8-36).

Gene expression of endogenous CEBPD, reporter CEBPD::SEAP, and CCL2 was significantly induced by M1 treatment, 3 and 6h after M1 treatment (Fig.8-36A, B, C, blue bars). The M1 treatment-mediated induction of endogenous CEBPD gene expression was completely abolished by vorinostat and TSA, while this of reporter CEBPD::SEAP was largely unaffected, at 1, 3, and 6h time points (Fig. 8-36A, B, red and orange bars). Vorinostat and TSA significantly reduced gene expression of CCL2 at all time points tested (Fig.8-36C, red and orange bars). Changes in SEAP secretion level were detected every 3 hours in cellular supernatants of non-polarized ( $M 0$ control) and M1 polarized THP-1 reporter $M \varphi$, for 24 hours. SEAP secretion was significantly upregulated by vorinostat and TSA at the later (18 to $24 \mathrm{~h}$ ) time points tested (Fig.8-36D, red and orange lines). 
A

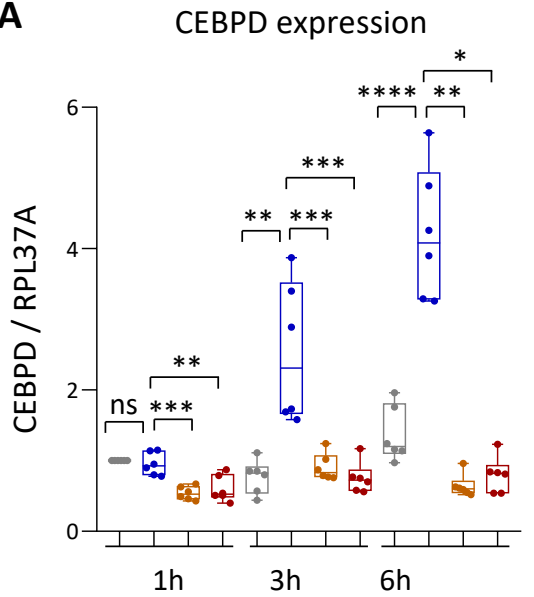

C

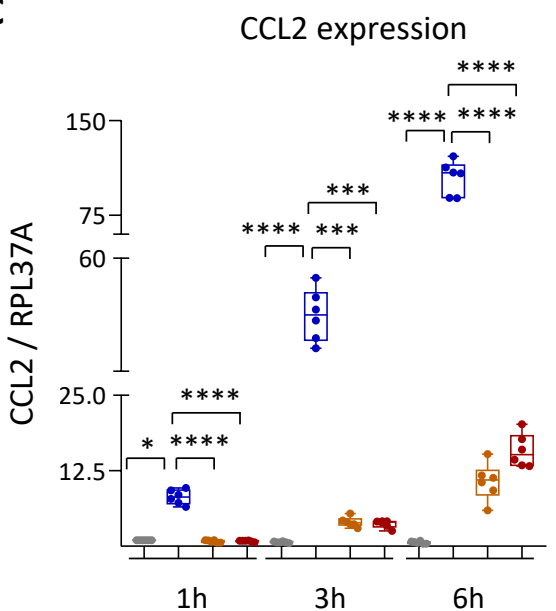

B CEBPD::SEAP expression

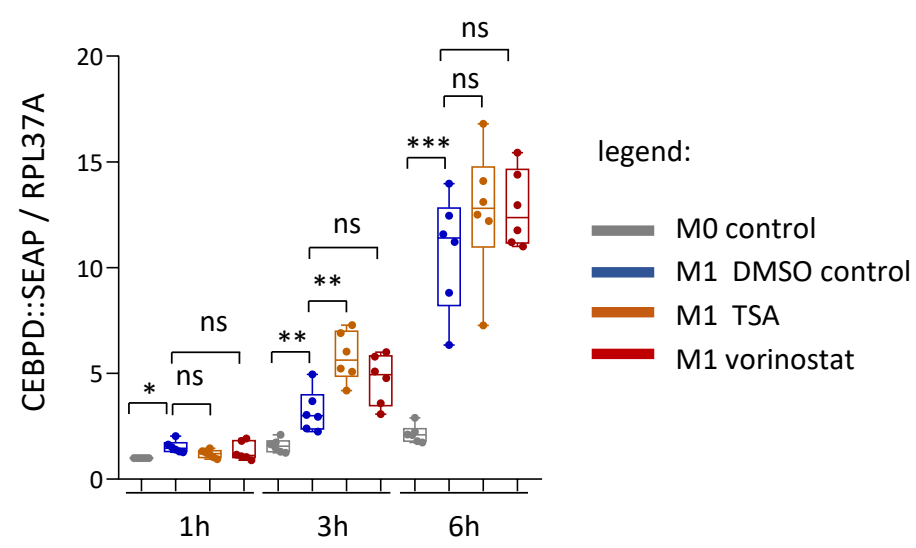

D

SEAP assay

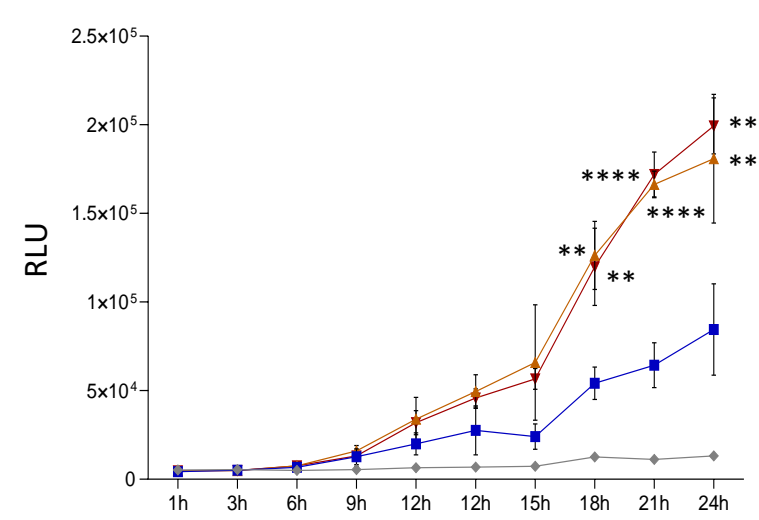

Figure 8-16: Effect of TSA and vorinostat on gene expression and SEAP secretion. Target THP-1 reporter cells were PMA-differentiated, seeded in a 24-well format, and treated as described previously (chapter 2.39.4). A, B, C: Changes in gene expression of endogenous CEBPD (A), reporter CEBPD::SEAP (B), and CCL2 (C) were analyzed in cell lysates of non-polarized (MO control) and M1-polarized DMSO-, TSA- (0.5 $\mu \mathrm{M})$, and vorinostat- $(10 \mu \mathrm{M})$ pre-treated reporter cells at indicated time points. Gene expression analysis was performed via RT-qPCR ( $\triangle \Delta C$ t method) using RPL37A as a house-keeping gene. Fold change in gene expression is displayed relative to minus M1 condition, detected one hour post-treatment and set as 1. M1 treatment-mediated induction in gene expression (difference between minus M1 and plus M1, DMSO) was analyzed via Wilcoxon signed-rank test (1.00 as hypothetical value). Compound-mediated changes in gene expression in comparison to DMSO were analyzed either ordinary one-way ANOVA, Brown-Forsythe and Welch ANOVA test with Dunnett's correction, or Kruskal-Wallis test with Dunn's correction for multiple comparisons. D: SEAP reporter secretion was monitored in cellular supernatants of corresponding reporter $M \varphi$, every $3 \mathrm{~h}$ over $24 \mathrm{~h}$. Changes in SEAP secretion were analyzed in comparison to DMSO control condition via ordinary one-way ANOVA Dunnett's correction or Kruskal-Wallis test with Dunn's correction for multiple comparisons. A, B, C, D: Data represent mean \pm SD of three single wells per condition, measured in duplicates. ${ }^{*} p<0.05 ;{ }^{* *} p<0.005 ;{ }^{* * *} p<0.001 ;{ }^{* * *} p<0.0001$; ns: not significant 


\subsection{Cloning strategies}

\subsubsection{Cloning strategy of pcDNA3.1(-)-G2P-based vectors}

HEK293T reporter cells stably expressing pcDNA3.1(-)-G2P-SEAP-myc and pcDNA3.1(-)G2P-Gaussia-myc vectors (Table 8.2) were generated to characterize SEAP and GLuc enzymatic assays (chapter 3.2.1.1). pcDNA3.1(-)-G2P served as a backbone (Table 8.1).

Table 8.1: Cloning of pcDNA3.1(-)-G2P vector.

\begin{tabular}{|c|c|}
\hline cloning strategy & standard, using restriction enzymes \\
\hline bacterial strains used & SCS110 \\
\hline \multicolumn{2}{|l|}{ backbone preparation } \\
\hline backbone, bacterial strain & pcDNA3.1(-) (Fig.8-17), SCS110 \\
\hline used restriction enzymes & BsaBI, BstBI \\
\hline digested backbone size & $4603 \mathrm{bp}$ \\
\hline \multicolumn{2}{|l|}{ insert preparation } \\
\hline template vector & pX335A-G2P (Fig.8-24) \\
\hline insert generation via & PCR, HD Advanced polymerase \\
\hline \multirow[t]{2}{*}{ primers } & F: G2P_BsaBI; R: G2P_BstBI \\
\hline & (Table 2.10) \\
\hline insert encodes & BsaBI-G2P-BstBI \\
\hline insert size & $1458 \mathrm{bp}$ \\
\hline digested insert size & 1437 bp \\
\hline ligation type & T4 ligase ligation \\
\hline transformation type & heat-shock, SCS110 bacteria \\
\hline selection antibiotic & $100 \mu \mathrm{g} / \mathrm{mL}$ ampicillin \\
\hline positive clone selection & restriction digestion after Holmes and Quigley prep \\
\hline \multirow[t]{2}{*}{ sequencing primers } & F: pcDNA_G2P_sv40; R: pcDNA_G2P \\
\hline & (Table 2.11$)$ \\
\hline final plasmid, size & pcDNA3.1(-)-G2P (Fig.8-29); 6,041 bp \\
\hline
\end{tabular}

Table 8.2: Cloning of pcDNA3.1(-)-G2P-GLuc-myc and pcDNA3.1(-)-G2P-SEAP-myc vectors.

cloning strategy standard, using restriction enzymes

bacterial strains used

SCS110, DH5 $\alpha$

backbone preparation

backbone, bacterial strain

pcDNA3.1(-)-G2P (Fig.8-29), SCS110

used restriction enzymes

BamHI-HF, HindIII-HF for Gaussia insert;

digested backbone size Xhol, HindIII-HF for SEAP insert

preparation of inserts

template vectors

pCMV-Gaussia (Fig.8-28) for Gaussia insert; 
insert generation via

primers

inserts encode

PCR-generated insert size

digested insert sizes

ligation type

transformation type

selection antibiotic

positive clone selection

sequencing primers

final plasmids, size
wt-SEAP-myc (Fig.8-18) for SEAP insert

PCR, HD Advanced polymerase for Gaussia-myc insert;

restriction digestion for SEAP-myc insert

F: Gaussia_myc; R: Gaussia_myc_HindIII

(Table 2.10)

BamHI-Gaussia-myc-HindIII *; SEAP-myc **

$613 \mathrm{bp} *$

$601 \mathrm{bp} *, 1563 \mathrm{bp} * *$

T4 ligase ligation

heat-shock, $\mathrm{DH} 5 \alpha$ bacteria

$100 \mu \mathrm{g} / \mathrm{mL}$ ampicillin

restriction digestion after Holmes and Quigley prep

F: seq_CMV_TB; F: seq_Gaussia; R: BGH_Poly

(Table 2.11)

pCDNA3.1(-)-G2P-GLuc-myc (Fig.8-30); 6,624 bp

pcDNA3.1(-)-G2P-SEAP-myc (Fig.8-31); 7,530 bp

\subsubsection{Cloning strategy of SP-SEAP-myc-NLS-mCherry vectors}

HEK293T reporter cells transiently expressing pmCherry-SP-SEAP-myc-NLS vectors were generated to evaluate SP-specific differences in protein targeting efficiency toward ER (chapter 3.2.2.1). SEAP-myc secreted reporter protein, carrying no (delta, $\Delta$ ), wild type (wt), AJAP-1 (shrew-1), prion (Prp), or prolactin (Prl) SPs, was N-terminally fused to a nuclear localization signal (NSL) and red fluorescent protein mCherry (Table 8.3). These vectors were cloned by Holger Jordan (Fraunhofer IME-TMP, Frankfurt am Main).

Table 8.3: Cloning of pmCherry-SP-SEAP-myc-NLS vectors.

\begin{tabular}{|c|c|}
\hline cloning strategy & standard, using restriction enzymes \\
\hline bacterial strains used & $\mathrm{DH} 5 \alpha$ \\
\hline \multicolumn{2}{|l|}{ backbone preparation } \\
\hline backbone, bacterial strain & pmCherry-N1 (Fig.8-27), DH5 $\alpha$ \\
\hline used restriction enzymes & Nhel, Kpnl \\
\hline digested backbone size & $4664 \mathrm{bp}$ \\
\hline \multicolumn{2}{|l|}{ insert preparation } \\
\hline template vectors & $\begin{array}{l}\text { wt-SEAP-myc (Fig.8-18), } \triangle \text { SP-SEAP-myc (Fig.8-19), } \\
\text { shrew-1- } \triangle \text { SP-SEAP-myc (Fig.8-20), Prl- } \Delta \text { SP-SEAP-myc } \\
\text { (Fig.8-21), Prp- } \triangle \text { SP-SEAP-myc (Fig.8-22) }\end{array}$ \\
\hline insert generation via & PCR, HD Advanced polymerase \\
\hline primers & F: uni_Nhel; R: myc_NLS_mCherry (Table 2.10) \\
\hline inserts encode & $\begin{array}{l}\text { wt-SEAP-myc-NLS (wt), } \Delta \text { SP-SEAP-myc-NLS }(\Delta) \text {, shrew- } \\
\text { 1-SEAP-myc-NLS (shrew-1), Prl-SEAP-myc-NLS (Prl), } \\
\text { Prp-SEAP-myc-NLS Prp) }\end{array}$ \\
\hline
\end{tabular}


insert sizes

digested insert sizes

ligation type

transformation type

selection antibiotic

positive clone selection

sequencing primers

final plasmids, size
1647 bp (wt), 1599 bp ( $\Delta$ ), 1744 bp (shrew-1), 1666 bp

(Prl), 1648 bp (Prp)

1613 bp (wt), 1565 bp ( $\Delta$ ), 1710 bp (shrew-1), 1632 bp

(Prl), 1614 bp (Prp)

T4 ligase ligation

heat-shock, $\mathrm{DH} 5 \alpha$ bacteria

$30 \mathrm{\mu g} / \mathrm{mL}$ kanamycin

restriction digestion after Holmes and Quigley prep

F: seq_CMV; R: seq-mCherry

(Table 2.11)

pmCherry-wt-SEAP-myc-NLS (Fig.8-32); 6,349 bp

pmCherry- $\triangle$ SEAP-myc-NLS (Fig.8-33); 6,231 bp

pmCherry-shrew-1-SEAP-myc-NLS (Fig.8-34); 6,376 bp

pmCherry-Prp-SEAP-myc-NLS (Fig.8-35); 6,280 bp

pmCherry-PrI-SEAP-myc-NLS (Fig.8-36); 6,298 bp

\subsubsection{Cloning strategy of pSEW-eGFP-based viral vectors encoding SEAP and GLuc}

THP-1 reporter cells stably expressing pSEW-eGFP-based vectors encoding SEAP and GLuc reporter proteins were generated to evaluate the use of SEAP and GLuc reporters in the screening assay (chapter 3.2.1.2) and were used to evaluate relative CEBPD promoter strength (chapter 8.3, Fig.8-6). THP-1 reporter cells stably expressing pSEW-eGFPCMV-GLuc-myc-CEBPD-SEAP-myc vector were used for screening. Final constructs (Table 8.5) were generated using intermediate vectors that express SEAP or GLuc under CMV promoter (Table 8.4), generated first.

Table 8.4: Cloning of pSEW-eGFP-CMV-SEAP-myc and pSEW-eGFP-CMV-Gaussia-myc vectors.

\begin{tabular}{ll}
\hline $\begin{array}{l}\text { cloning strategy } \\
\text { bacterial strains used }\end{array}$ & $\begin{array}{l}\text { standard, using restriction enzy } \\
\text { SCS110, DH5 } \alpha\end{array}$ \\
backbone preparation & \\
backbone, bacterial strain & pSEW-eGFP (Fig.8-23), SCS110 \\
used restriction enzymes & Kfll, EcoRI \\
digested backbone size & $9373 \mathrm{bp}$
\end{tabular}

insert preparation

template vector

pcDNA3.1(-) (Fig.8-17) for CMV promoter, pcDNA3.1(-

)-G2P-GLuc-myc (Fig.8-31) for Gaussia-myc, pcDNA3.1(-)-G2P-SEAP-myc (Fig.8-30) for SEAP-myc

inserts generation via two-round PCR, HD Advanced polymerase

first round PCR

primers

F: Kfll-CMV-Space; R: Space-SPg; F: Space_SPg; R: BGH poly EcoRI; R: Space-SPs; F: Space_SPs 


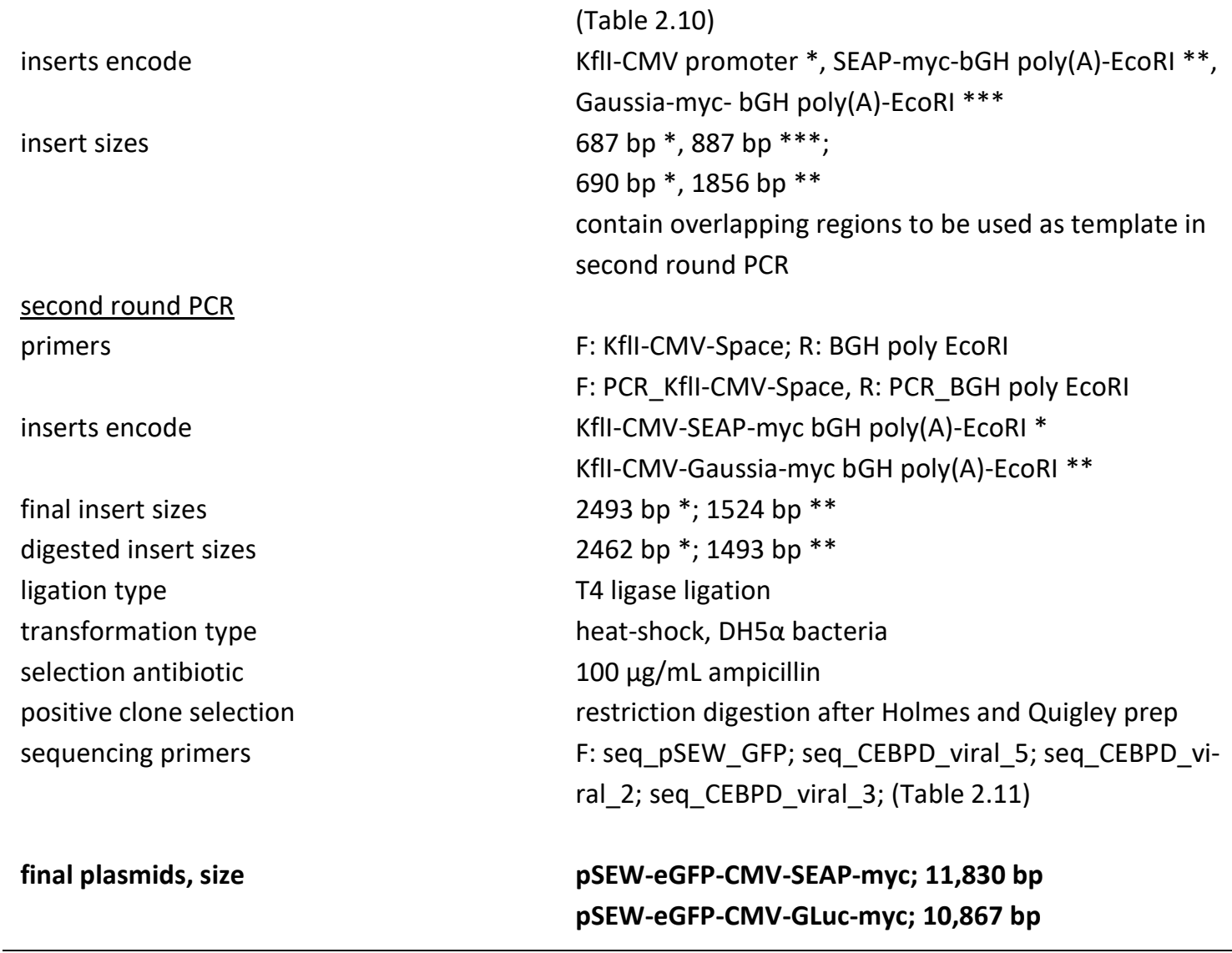

Table 8.5: Cloning of pSEW-eGFP-CMV-GLuc-myc-CEBPD-SEAP-myc and pSEW-eGFP-CMV-SEAP-mycCEBPD-GLuc-myc constructs.

\begin{tabular}{|c|c|}
\hline cloning strategy & standard, using restriction enzymes \\
\hline bacterial strains used & $\mathrm{DH} 5 \alpha$ \\
\hline \multicolumn{2}{|l|}{ backbone preparation } \\
\hline \multirow[t]{2}{*}{ backbones, bacterial strain } & pSEW-eGFP-CMV-SEAP-myc *, DH5 $\alpha$ \\
\hline & pSEW-eGFP-CMV-GLuc-myc **, DH5 $\alpha$ \\
\hline used restriction enzymes & Xhol, Acc65l \\
\hline \multirow[t]{2}{*}{ digested backbone sizes } & $11,811 \mathrm{bp} *$ \\
\hline & $10,848 \mathrm{bp} * *$ \\
\hline \multicolumn{2}{|l|}{ insert preparation } \\
\hline \multirow[t]{4}{*}{ template vector } & pcDNA3.1(-) (Fig.8-17) for SV40 early polyA, \\
\hline & pcDNA3.1(-)-G2P-GLuc-myc (Fig.8-31) for Gaussia-myc, \\
\hline & pcDNA3.1(-)-G2P-SEAP-myc (Fig.8-30) for SEAP-myc, \\
\hline & BAC clone $\mathrm{CH} 17-293 \mathrm{~N} 3$ for CEBPD promoter \\
\hline inserts generation via & three-round PCR, HD Advanced polymerase \\
\hline \multicolumn{2}{|l|}{ first round PCR } \\
\hline \multirow[t]{3}{*}{ primers } & F: Xhol SV40 poly; R: SV40 poly BGH poly; F: gaussia \\
\hline & backbone-CEBPD; R: SEAP-myc-CEBPD \\
\hline & (Table 2.10) \\
\hline inserts encode & Xhol-SV40 early poly(A), CEBPD promoter \\
\hline
\end{tabular}


second round PCR

primers

inserts encode

third round PCR

primers

inserts encode

final insert sizes

digested insert sizes

ligation type

transformation type

selection antibiotic

positive clone selection

sequencing primers

final plasmids, size

\author{
F: BGH poly SV40 poly; R: Acc65I CEBPD sv40 \\ Acc65I-CEBPD-SEAP-myc-bGH poly(A); \\ Acc65I-CEBPD-Gaussia-myc-bGH poly(A)
}

F: Xhol SV40 poly; R: Acc65I CEBPD sv40

Acc65I-CEBPD-SEAP-myc-bGH poly(A)- SV40 early

$\operatorname{poly}(A)-X h o l *$;

Acc65I-CEBPD-Gaussia-myc-bGH poly(A) SV40 early

$\operatorname{poly}(\mathrm{A})-\mathrm{Xhol} * *$

$2303 \mathrm{bp} *, 1340 \mathrm{bp} * *$

2286 bp *, 1329 bp **

T4 ligase ligation

heat-shock, $\mathrm{DH} 5 \alpha$ bacteria

$100 \mu \mathrm{g} / \mathrm{mL}$ ampicillin

restriction digestion after Holmes and Quigley prep

seq_CEBPD_viral_4; seq_CEBPD_viral_3; seq_CE-

BPD_viral_2; R: qPCR_SEAP_WT; seq_CEBPD_viral_6;

R: PCR_SEAP_space

(Table 2.11)

pSEW-eGFP-CMV-GLuc-myc-CEBPD-SEAP-myc

(Fig.8-37); 13,134 bp; pSEW-eGFP-CMV-SEAP-myc-CE-

BPD-GLuc-myc (Fig.8-38); 13,140 bp

\subsubsection{Cloning strategy of the multi-gene-reporter cassette 2.0 in pcDNA3.1(-) backbone}

The multi-gene-reporter cassette 2.0 was constructed in pcDNA3.1(-) backbone, which is easy to propagate in $\mathrm{DH} 5 \alpha$ E.coli as a high-copy plasmid. Due to its structural complexity, multi-gene-reporter cassette 2.0 was cloned over three steps: i) generation of pcDNA3.1(-)-CMV-GLuc-myc vector (Table 8.6); ii) generation of pcDNA3.1(-)-H2G-NLSCMV-GLuc-myc vector (Table 8.7); iii) generation of pcDNA3.1(-)-Prp-SEAP-myc-NLSH2G-NLS-CMV-GLuc-myc vector (Table 8.8).

Table 8.6: Cloning of pcDNA3.1(-)-CMV-GLuc-myc vector.

\begin{tabular}{ll}
\hline $\begin{array}{l}\text { cloning strategy } \\
\text { bacterial strains used }\end{array}$ & $\begin{array}{l}\text { standard, using restriction enzymes } \\
\mathrm{DH} 5 \alpha\end{array}$ \\
$\begin{array}{l}\text { backbone preparation } \\
\text { backbone, bacterial strain } \\
\text { used restriction enzymes } \\
\text { digested backbone size }\end{array}$ & pcDNA3.1(-) (Fig.8-17),DH5 $\alpha$ \\
& Xhol, EcoRV \\
insert preparation & 5402 bp \\
template vector & \\
\end{tabular}


inserts generation via

primers

inserts encode

insert size

digested insert size

ligation type

transformation type

selection antibiotic

positive clone selection

sequencing primer
PCR, Q5 High-Fidelity DNA polymerase

F: Xhol_pA_GLuc; R: EcoRV_CMV

(Table 2.10)

EcoRV-CMV-GLuc-myc-Xhol

$1497 \mathrm{bp}$

$1482 \mathrm{bp}$

NEB Quick ligation

heat-transformation

$100 \mu \mathrm{g} / \mathrm{mL}$ ampicillin

colony PCR

F: PGK_bGH; R: EcoRV_CMV

(Table 2.31)

restriction digestion after Holmes and Quigley prep

F: qPCR_Gaussia; R: qPCR_Gaussia

(Table 2.11)

pcDNA3.1(-)-CMV-GLuc-myc; 6,884 bp

Table 8.7: Cloning of pcDNA3.1(-)-H2G-NLS-CMV-GLuc-myc vector.

\begin{tabular}{|c|c|}
\hline cloning strategy & assembly \\
\hline bacterial strains used & $\mathrm{DH} 5 \alpha$ \\
\hline \multicolumn{2}{|l|}{ backbone preparation } \\
\hline backbone, bacterial strain & pcDNA3.1(-)-CMV-GLuc-myc, DH5 $\alpha$ \\
\hline used restriction enzymes & Xhol \\
\hline digested backbone size & $6884 \mathrm{bp}$ \\
\hline \multicolumn{2}{|l|}{ insert preparation } \\
\hline \multirow[t]{3}{*}{ template vector } & pcDNA3.1(-)-G2P (Fig.8-29) for copGFP and bGH \\
\hline & $\operatorname{poly}(A)$ \\
\hline & $\begin{array}{l}\text { pR6k(hygro)-bglobin (Fig.8-25) for hygromycin re- } \\
\text { sistance }\end{array}$ \\
\hline inserts generation via & three-round PCR, Q5 High-Fidelity DNA polymerase \\
\hline \multicolumn{2}{|l|}{ first round PCR } \\
\hline \multirow[t]{4}{*}{ primers } & F: T2A_hygro; R: bGH_PGK; F: pcDNA_G2H; R: \\
\hline & NLS_bGH; F: bGH_NLS_cGFP; R: GFP_NLS; R: \\
\hline & T2A_cGFP \\
\hline & (Table 2.10) \\
\hline \multirow[t]{2}{*}{ inserts encode } & PGK-em7-hygromysin-T2A *, bGH poly(A) **, copGFP- \\
\hline & NLS $* * * ;$ with overlapping regions \\
\hline insert sizes & 1685 bp *, 353 bp **, 844 bp *** \\
\hline \multicolumn{2}{|l|}{ second round PCR } \\
\hline primers & F: bGH_NLS; R: bGH_PGK \\
\hline insert encodes & PGK-em7-hygromysin-T2A-copGFP-NLS \\
\hline insert size & $2498 \mathrm{bp}$ \\
\hline
\end{tabular}


primers

insert encodes

insert size

ligation type

transformation type

selection antibiotic

positive clone selection

sequencing primer

final plasmid, size
F: pcDNA_G2H; R: bGH_PGK

PGK-em7-hygromysin-T2A- copGFP-NLS-bGH poly(A)

2812 bp

NEB Assembly

heat-transformation

$100 \mu \mathrm{g} / \mathrm{mL}$ ampicillin, $50 \mu \mathrm{g} / \mathrm{mL}$ hygromycin

colony $\mathrm{PCR}$

F: Xho_pA_GLuc; R: bGH_PGK

(Table 2.15)

F: pcDNA_G2H; R: bGH_PGK; R: T2A_cGFP; F: T2A_hy-

gro

(Table 2.11)

pcDNA3.1(-)-H2G-NLS-CMV-GLuc-myc (Fig.8-39);

9,649 bp

Table 8.8: Cloning of pcDNA3.1(-)-Prp-SEAP-myc-NLS-H2G-NLS-CMV-GLuc-myc vector.

\begin{tabular}{|c|c|}
\hline cloning strategy & assembly \\
\hline bacterial strains used & $\mathrm{DH} 5 \alpha$ \\
\hline \multicolumn{2}{|l|}{ backbone preparation } \\
\hline backbone, bacterial strain & pcDNA3.1(-)-H2G-NLS-CMV-GLuc-myc (Fig.8-39), DH5 $\alpha$ \\
\hline used restriction enzymes & Xhol, Nhel-HF \\
\hline digested backbone size & 9623 bp \\
\hline \multicolumn{2}{|l|}{ insert preparation } \\
\hline template vector & pmCherry-Prp-SEAP-myc-NLS (Fig.8-35) \\
\hline inserts generation via & PCR, Q5 High-Fidelity DNA polymerase \\
\hline \multirow[t]{2}{*}{ primers } & F: pcDNA_Prp; R: SV40_Poly_pcDNA \\
\hline & (Table 2.10$)$ \\
\hline inserts encode & $\begin{array}{l}\text { Prp-SEAP-myc-NLS-mCherry-SV40 poly(A), with over- } \\
\text { lapping regions on backbone }\end{array}$ \\
\hline insert size & $2623 \mathrm{bp}$ \\
\hline ligation type & NEB Assembly \\
\hline transformation type & heat-transformation \\
\hline selection antibiotic & $100 \mu \mathrm{g} / \mathrm{mL}$ ampicillin, $50 \mu \mathrm{g} / \mathrm{mL}$ hygromycin \\
\hline \multirow[t]{3}{*}{ positive clone selection } & colony PCR \\
\hline & F: Nhe_Prp_SEAP; R: Xho_SV40 \\
\hline & (Table 2.15) \\
\hline \multirow[t]{3}{*}{ sequencing primer } & R: SV40_Poly_pcDNA; R: seq-mCherry; F: \\
\hline & qPCR_SEAP_WT; R: qPCR_SEAP_WT \\
\hline & (Table 2.11) \\
\hline \multirow[t]{2}{*}{ final plasmid, size } & pcDNA3.1(-)-Prp-SEAP-myc-NLS-H2G-NLS-CMV-GLuc- \\
\hline & myc (Fig.8-40); 12,222 bp \\
\hline
\end{tabular}




\subsubsection{Cloning strategy of multi-gene-reporter cassette 2.0 in pR6k backbone}

To generate a reporter BAC vector via homolog recombination, it is obligatory to use pR6k-based vector as a template for the insert generation via PCR (chapter 2.25.2). The Prp-SEAP-myc-NLS-H2G-NLS-CMV-GLuc-myc vector was generated (Table 8.9) encoding the constructed multi-gene-reporter cassette 2.0 in a pR6k backbone.

Table 8.9: Cloning of pR6k-Prp-SEAP-myc-NLS-H2G-NLS-CMV-GLuc-myc vector.

\begin{tabular}{|c|c|}
\hline cloning strategy & in vivo assembly \\
\hline bacterial strains used & PIR1 \\
\hline \multicolumn{2}{|l|}{ backbone preparation } \\
\hline backbone, bacterial strain & pR6k(hygro)-bglobin (Fig.8-25) \\
\hline used restriction enzymes & Sacl-HF, Bmtl-HF \\
\hline digested backbone size & $4785 \mathrm{bp}$ \\
\hline \multicolumn{2}{|l|}{ insert preparation } \\
\hline template vector & $\begin{array}{l}\text { pcDNA3.1(-)-Prp-SEAP-myc-NLS-H2G-NLS-CMV-GLuc- } \\
\text { myc (Fig.8-40) }\end{array}$ \\
\hline inserts generation via & PCR, AccuPrime PfxSuperMix \\
\hline \multirow[t]{2}{*}{ primers } & F: Bmtl_GLuc; R: Prp_Sacl \\
\hline & (Table 2.10$)$ \\
\hline \multirow[t]{3}{*}{ inserts encode } & Prp-SEAP-myc-NLS-mCherry-SV40 poly(A)-bGH \\
\hline & poly(A)-H2G-NLS-CMV-GLuc-myc-bGH poly(A), with \\
\hline & overlapping regions on backbone \\
\hline insert size & $6893 \mathrm{bp}$ \\
\hline ligation type & in vivo assembly \\
\hline transformation type & heat-transformation \\
\hline selection antibiotic & $100 \mu \mathrm{g} / \mathrm{mL}$ ampicillin, $50 \mu \mathrm{g} / \mathrm{mL}$ hygromycin \\
\hline \multirow[t]{3}{*}{ positive clone selection } & colony PCR \\
\hline & F: Nhe_Prp_SEAP; R: Xho_SV40 \\
\hline & (Table 2.15) \\
\hline \multirow[t]{3}{*}{ sequencing primer } & R: SV40_Poly_pcDNA; R: seq-mCherry; F: \\
\hline & qPCR_SEAP_WT; R: qPCR_SEAP_WT \\
\hline & (Table 2.11) \\
\hline \multirow[t]{2}{*}{ final plasmid, size } & pR6k-Prp-SEAP-myc-NLS-H2G-NLS-CMV-GLuc-myc, \\
\hline & (Fig.8-41); 11,632 bp \\
\hline
\end{tabular}

\subsubsection{Cloning strategy of multi-gene-reporter cassette 2.0 under control of CEBPD pro- moter in pcDNA3.1(-) backbone}

HEK293T reporter cells transiently expressing the multi-gene-reporter cassette 2.0 were generated to test its functionality in-vitro (chapter 3.2.2.2). To do so, the multi-gene- 
reporter cassette 2.0 was set under control of the defined CEBPD promoter and expressed on the pcDNA3.1(-) backbone (Table 8.10).

Table 8.10: Cloning of pcDNA3.1(-)-CEBPD-Prp-SEAP-myc-NLS-H2G-NLS-CMV-GLuc-myc vector.

\begin{tabular}{|c|c|}
\hline cloning strategy & NEB assembly \\
\hline bacterial strains used & $\mathrm{DH} 5 \alpha$ \\
\hline \multicolumn{2}{|l|}{ backbone preparation } \\
\hline \multirow[t]{2}{*}{ backbone, bacterial strain } & pcDNA3.1(-)-Prp-SEAP-myc-NLS-H2G-NLS-CMV-GLuc- \\
\hline & myc (Fig.8-40), DH5 $\alpha$ \\
\hline used restriction enzymes & Sspl-HF, Bmtl-HF \\
\hline digested backbone size & $11,204 \mathrm{bp}$ \\
\hline \multicolumn{2}{|l|}{ insert preparation } \\
\hline \multirow{2}{*}{ template vector } & pSEW-eGFP-CMV-GLuc-myc-CEBPD-SEAP-myc \\
\hline & (Fig.8-37) \\
\hline inserts generation via & PCR, AccuPrime PfxSuperMix \\
\hline \multirow[t]{2}{*}{ primers } & F:PCR_CEBPD_cassette; R: PCR_CEBPD_cassette \\
\hline & (Table 2.10$)$ \\
\hline \multirow[t]{2}{*}{ inserts encode } & CEBPD promoter, with overlapping regions on back- \\
\hline & bone \\
\hline insert size & $385 \mathrm{bp}$ \\
\hline ligation type & NEB Assembly \\
\hline transformation type & heat-transformation \\
\hline selection antibiotic & $100 \mu \mathrm{g} / \mathrm{mL}$ ampicillin, $50 \mu \mathrm{g} / \mathrm{mL}$ hygromycin \\
\hline \multirow[t]{4}{*}{ positive clone selection } & colony PCR \\
\hline & F:PCR_CEBPD_cassette; R: qPCR_SEAP_WT \\
\hline & (Table 2.15) \\
\hline & test restriction digestion after plasmid DNA mini prep \\
\hline \multirow[t]{5}{*}{ sequencing primer } & R: SV40_Poly_pcDNA; R: seq-mCherry; F: \\
\hline & qPCR_SEAP_WT; R: qPCR_SEAP_WT; R: bGH_PGK; R: \\
\hline & T2A_cGFP; F: T2A_hygro; F: seq_BAC_4; F: \\
\hline & seq_test_BAC_2; F: qPCR_Gaussia; R: qPCR_Gaussia \\
\hline & (Table 2.11) \\
\hline \multirow[t]{2}{*}{ final plasmid, size } & pcDNA3.1(-)-CEBPD-Prp-SEAP-myc-NLS-H2G-NLS- \\
\hline & CMV-GLuc-myc (Fig.8-42); 11,538 bp \\
\hline
\end{tabular}




\subsection{Plasmid maps}

Below are shown plasmid maps (Fig.8-17 to 8-42) of in Table 2.12 and 2.13 (chapter 2.9)

listed plasmids. Plasmid maps show only sequences, which were relevant for cloning.

\section{List of abbreviations.}

\begin{tabular}{ll}
\hline abbreviation & meaning \\
\hline ampicillinR & ampicillin resistance gene \\
AraC & arabinose operon regulatory protein \\
bGH poly(A) & bovine growth hormone polyadenylation signal \\
CMV enhancer & cytomegalovirus enhancer \\
CMV promoter & cytomegalovirus promoter \\
copGFP & copepod green fluorescent protein \\
eGFP & enhanced green fluorescent protein \\
em 7 promoter & bacterial em7 promoter \\
FRT & flippase recognition target \\
hygromycinR & hygromycin resistance gene \\
MCS & multiple cloning site \\
neomycin/kanamycinR & neomycin and kanamycin resistance gene \\
NLS & nuclear localization signal \\
PGK promoter & phosphoglycerate kinase promoter \\
puromycinR & puromycin resistance gene \\
R6kg_origin & origin of replication for pR6k plasmids \\
RecA & Protein for repair and maintenance of bacterial DNA \\
RRE & HIV-1 Rev response element \\
SFFVp promoter & lenti-viral promoter \\
SP & signal peptide \\
T2A & self-cleavage peptide \\
T7 promoter & phage T7 promoter \\
tetracyclinR & tetracycline resistance gene \\
viral regulatory sequences & 3A'SIN, psi, PBS, U5, R, 5'LTR \\
WPRE & Woodchuck Hepatitis Virus (WHP) posttranscriptional regulatory ele- \\
& ment \\
\hline
\end{tabular}




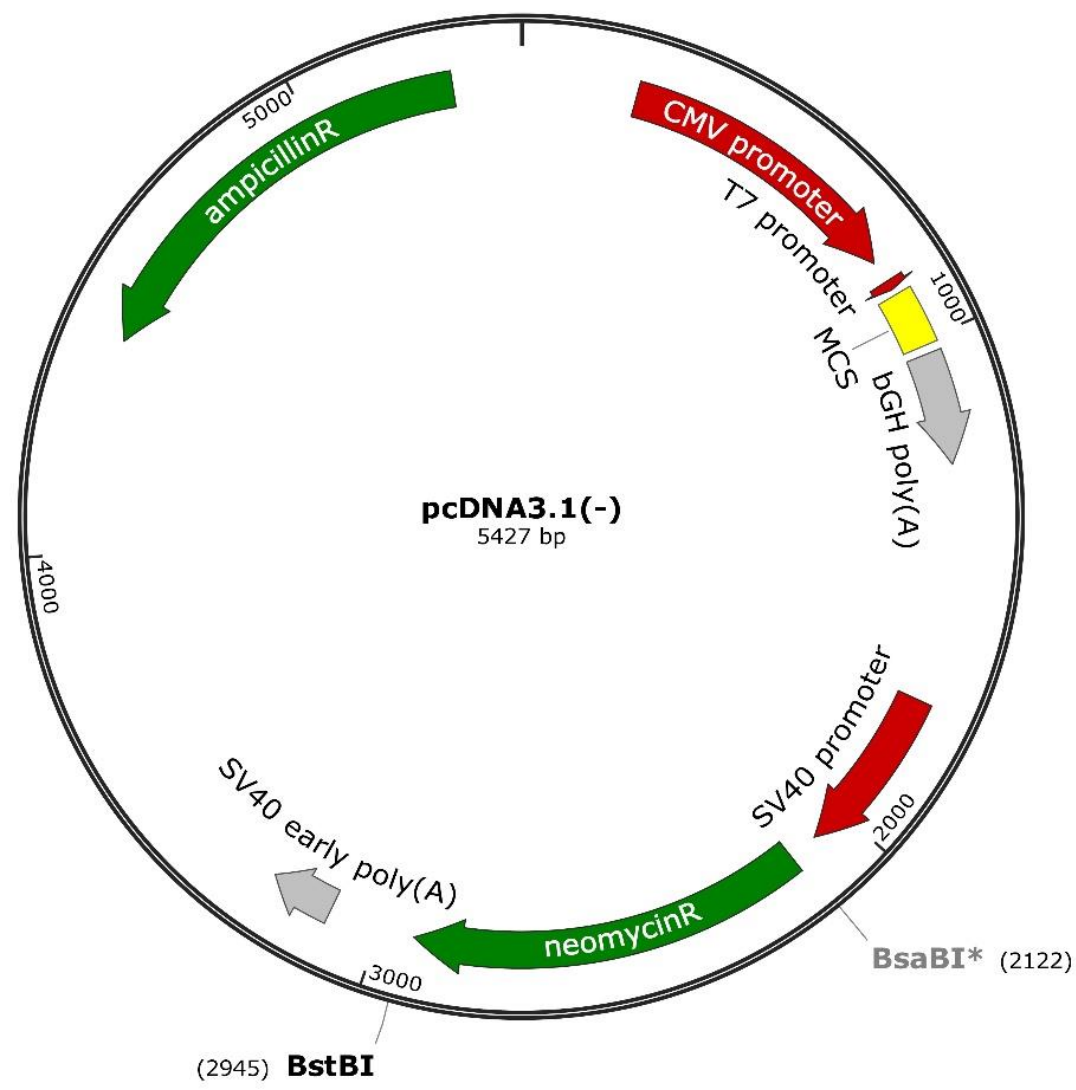

Figure 8-17: Plasmid map of pcDNA3.1(-) backbone vector.

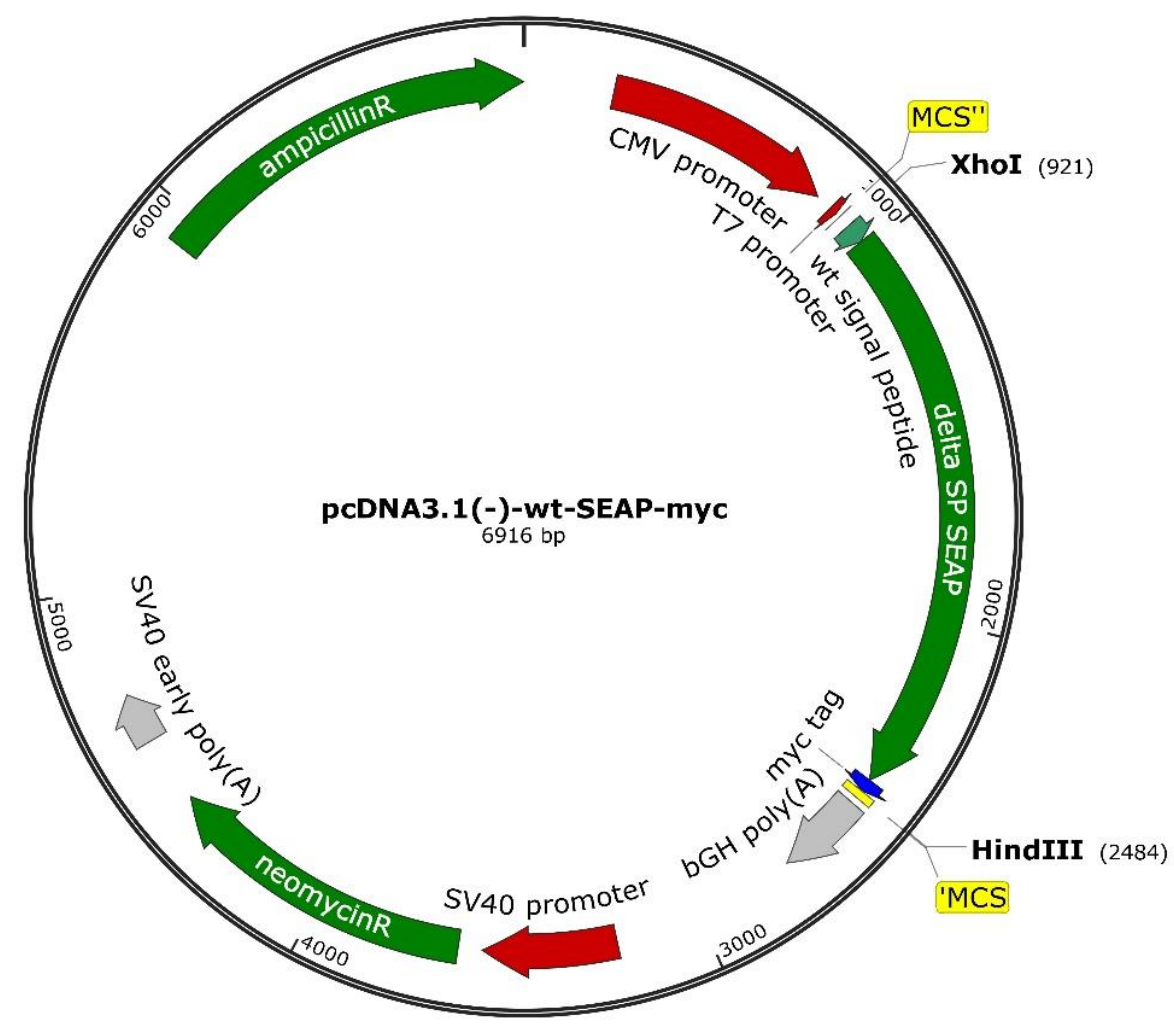

Figure 8-18: Plasmid map of wt-SEAP-myc vector in pcDNA3.1(-) backbone. 


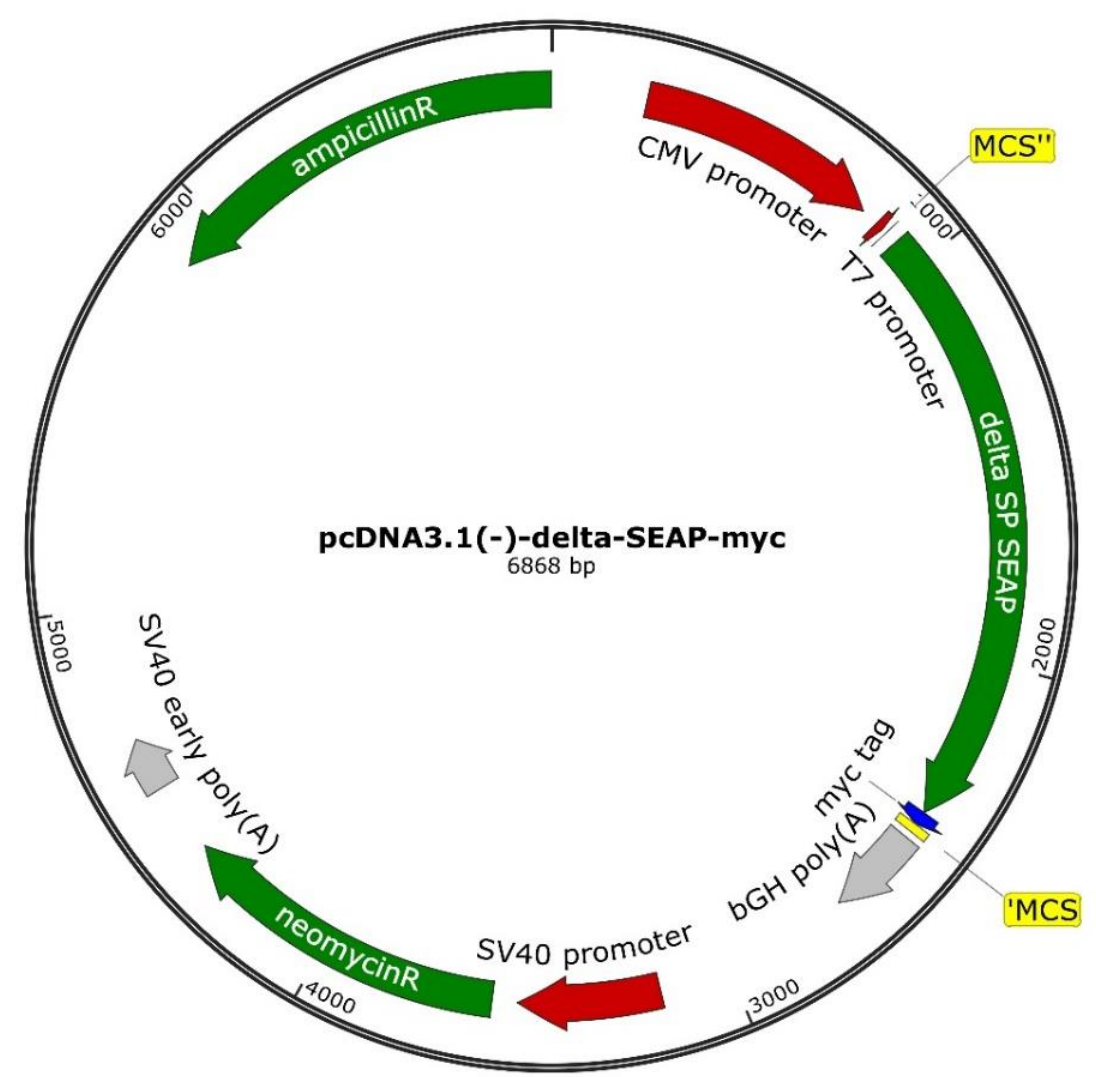

Figure 8-19: Plasmid map of $\triangle$ SP-SEAP-myc vector in pcDNA3.1(-) backbone.

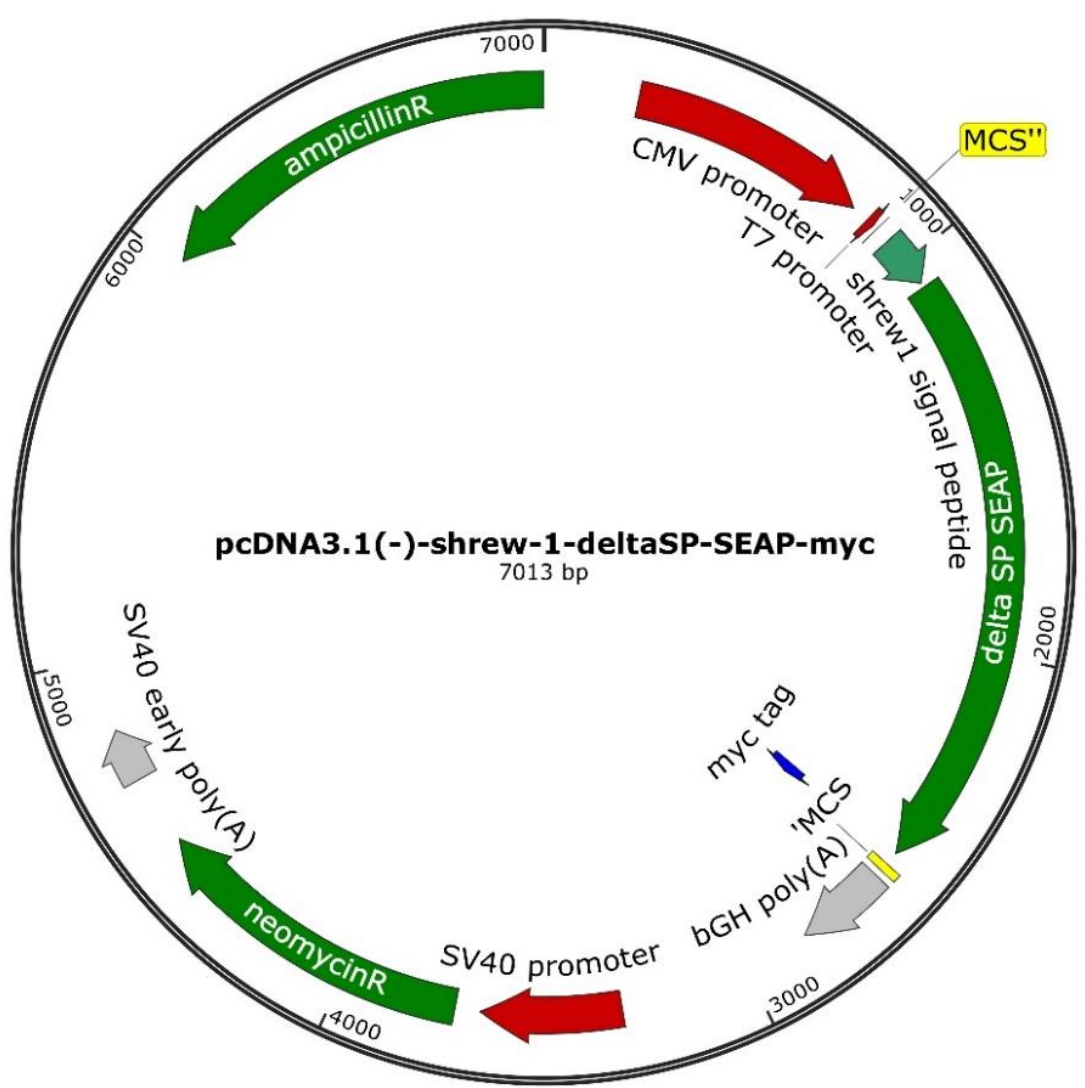

Figure 8-20: Plasmid map of shrew-1- $\triangle$ SP-SEAP-myc vector in pcDNA3.1(-) backbone. 


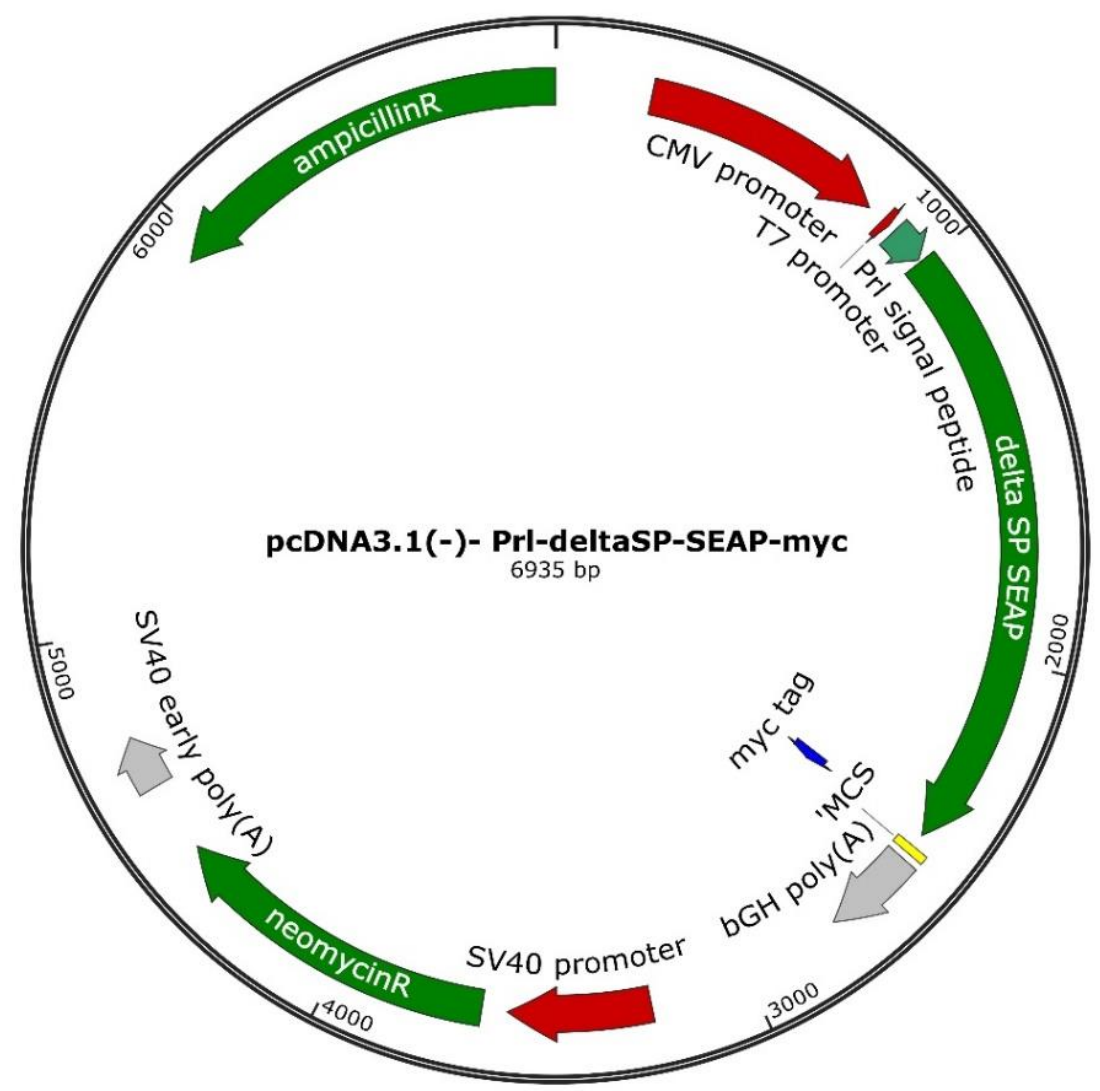

Figure 8-21: Plasmid map of Prl- $\triangle$ SP-SEAP-myc vector in pcDNA3.1(-) backbone.

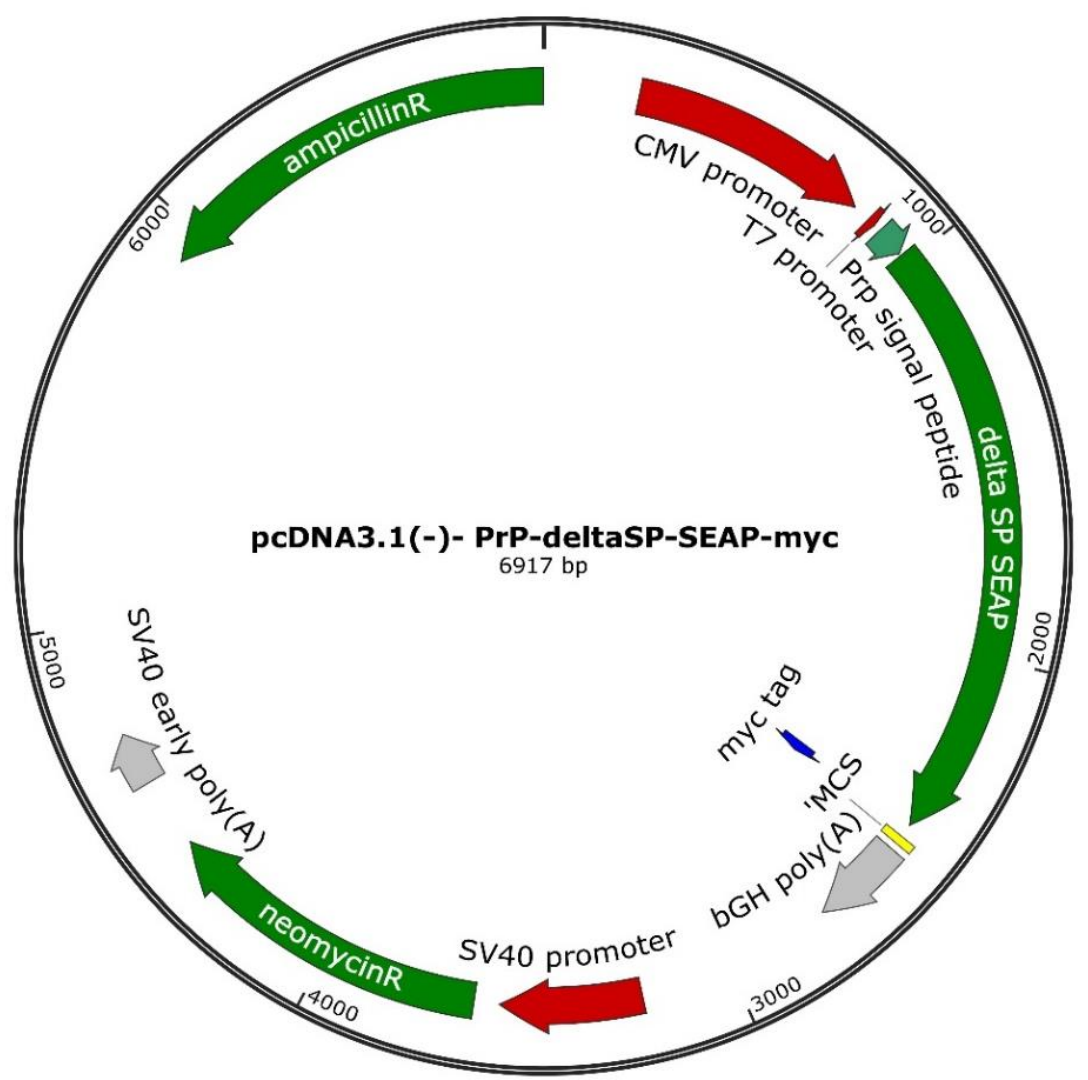

Figure 8-22: Plasmid map of Prp-DSP-SEAP-myc vector in pcDNA3.1(-) backbone. 


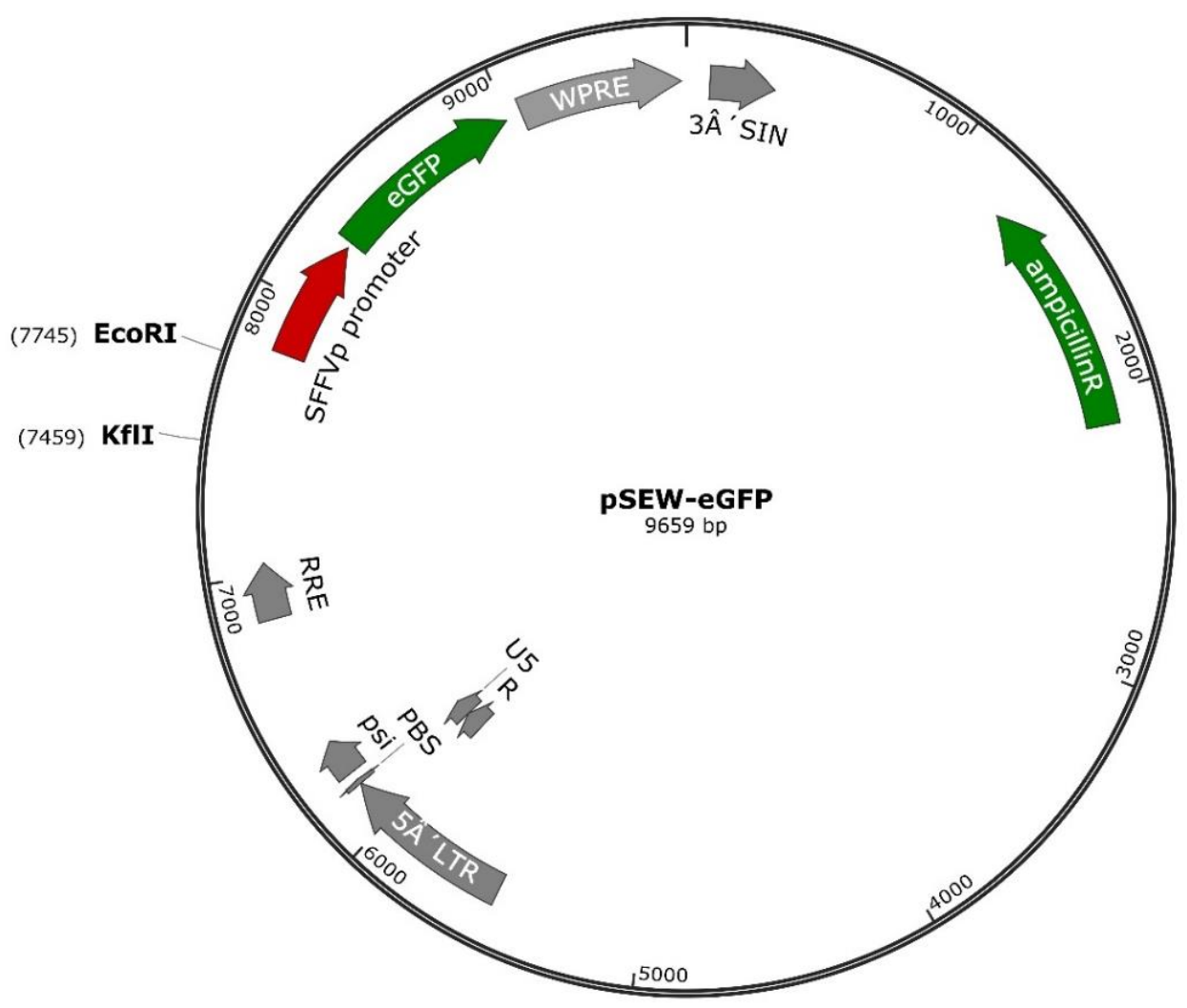

Figure 8-23: Plasmid map of pSEW-eGFP backbone vector.

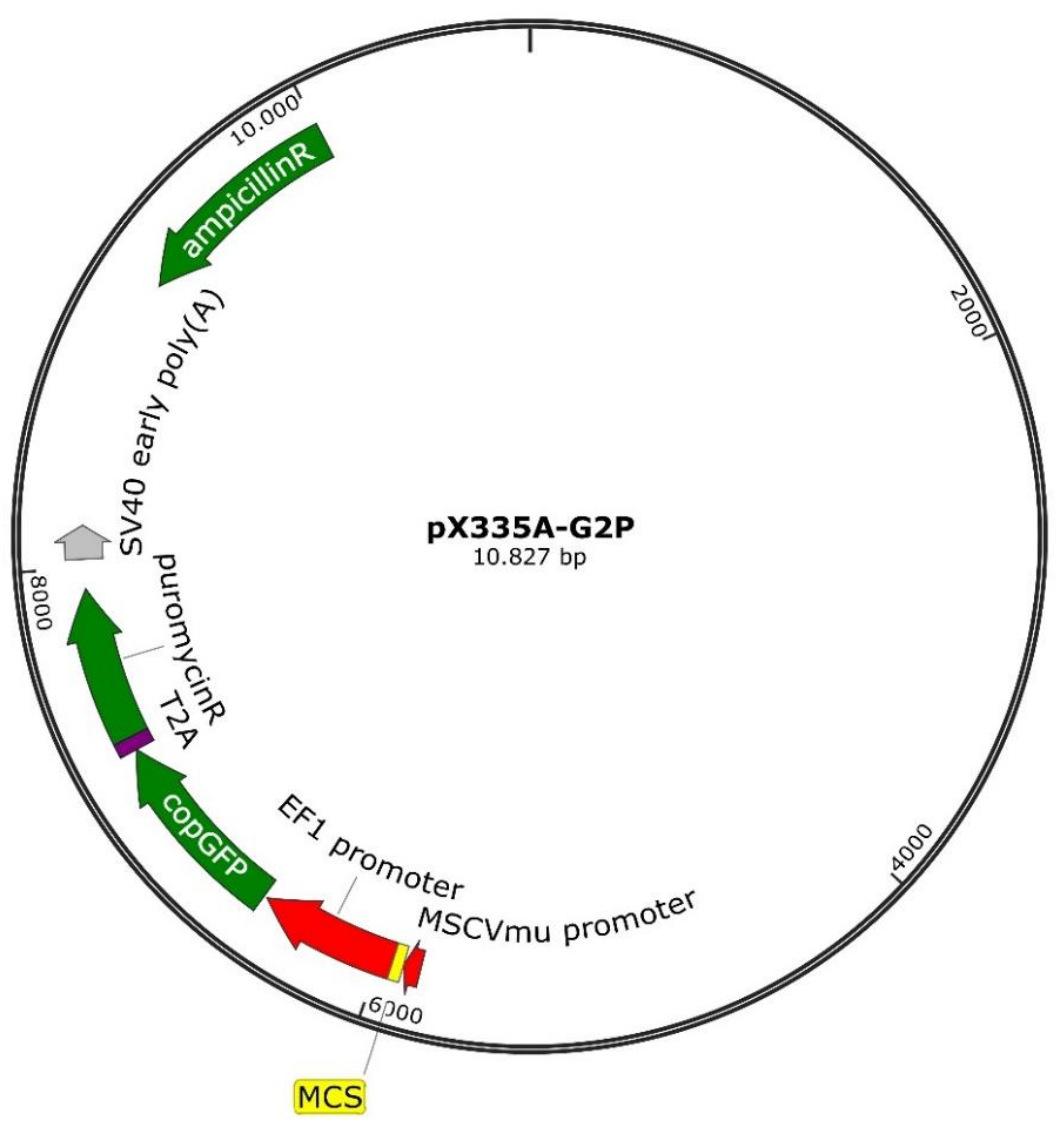

Figure 8-24: Plasmid map of pX335A-G2P vector. 


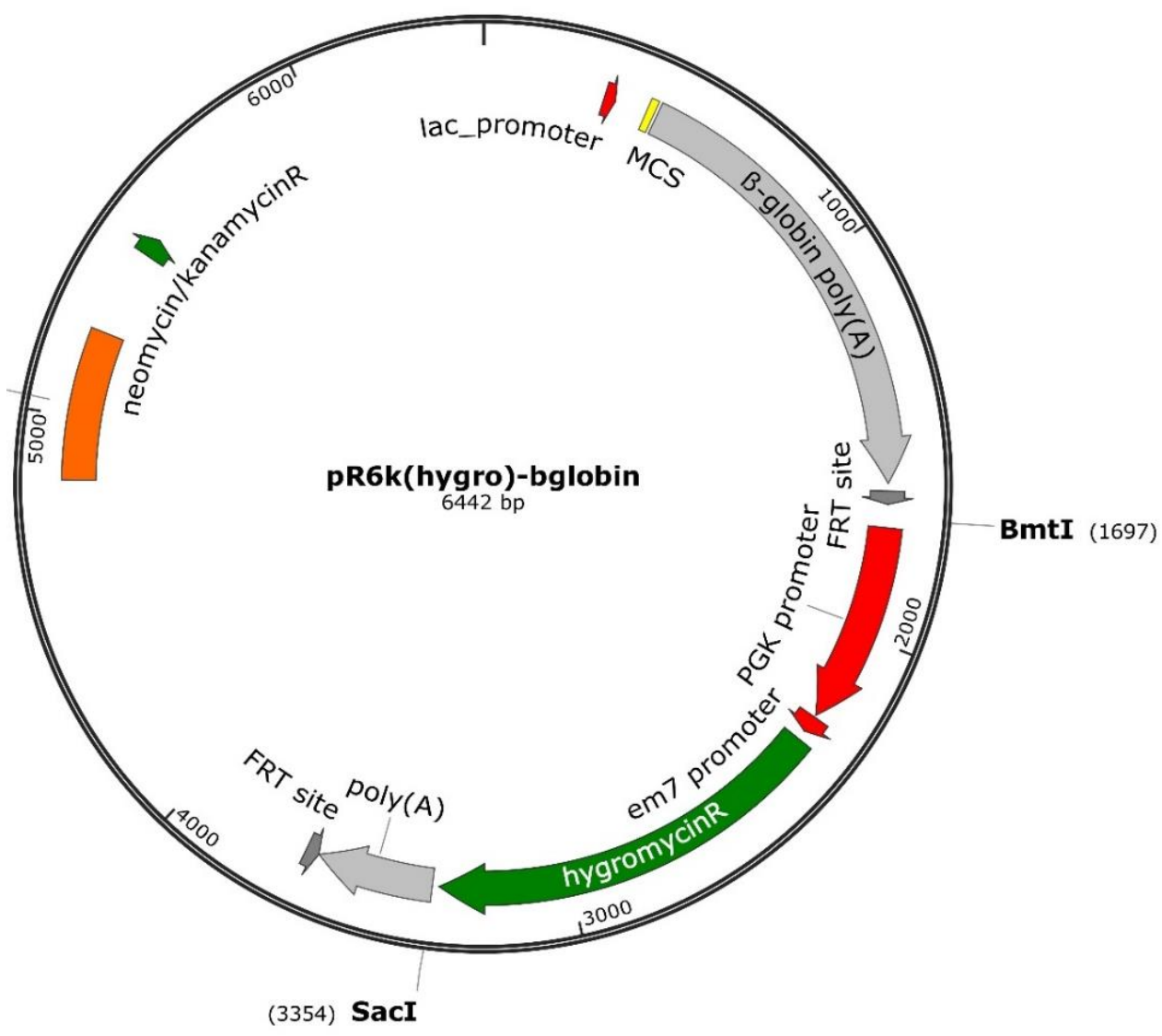

Figure 8-25: Plasmid map of pR6k(hygro)-bglobin vector.

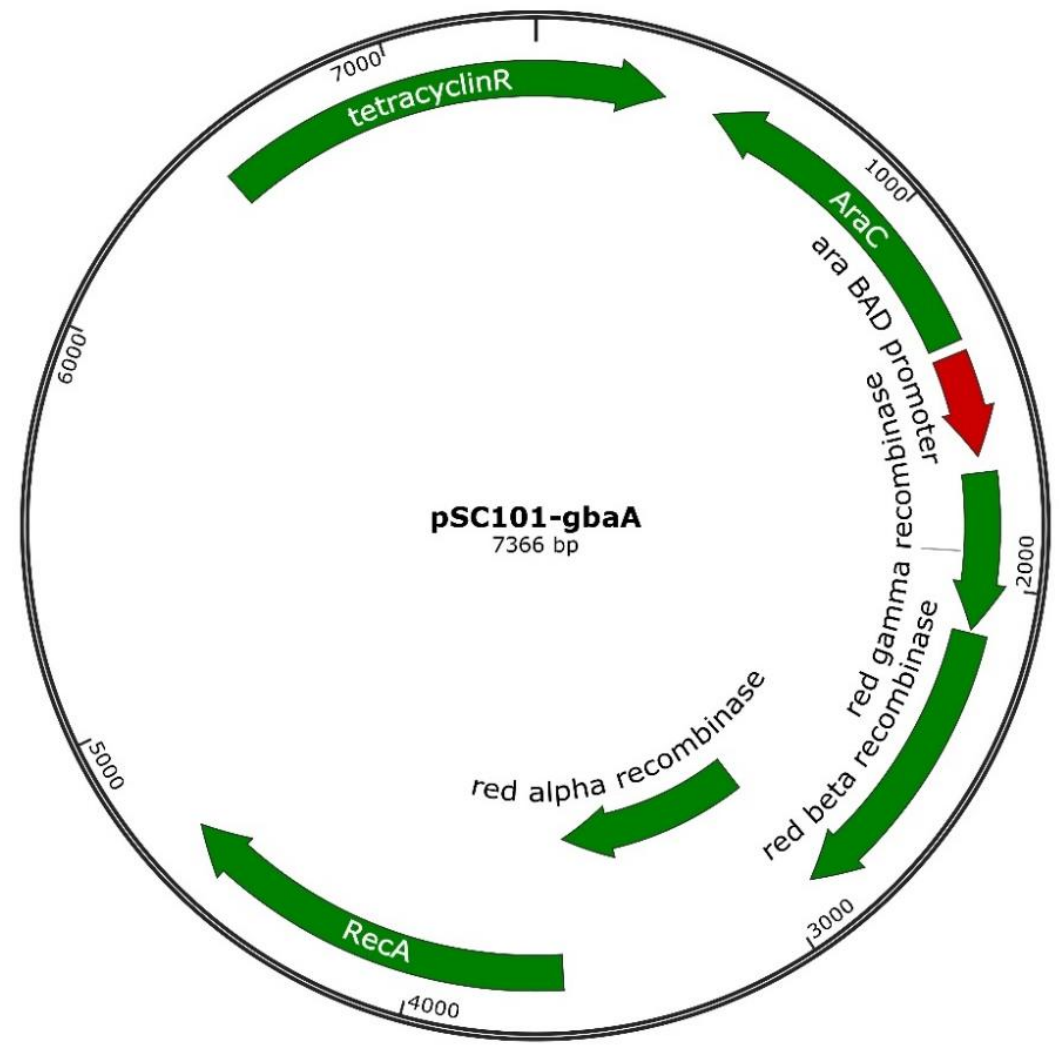

Figure 8-26: Plasmid map of pSC101-gbaA vector. 


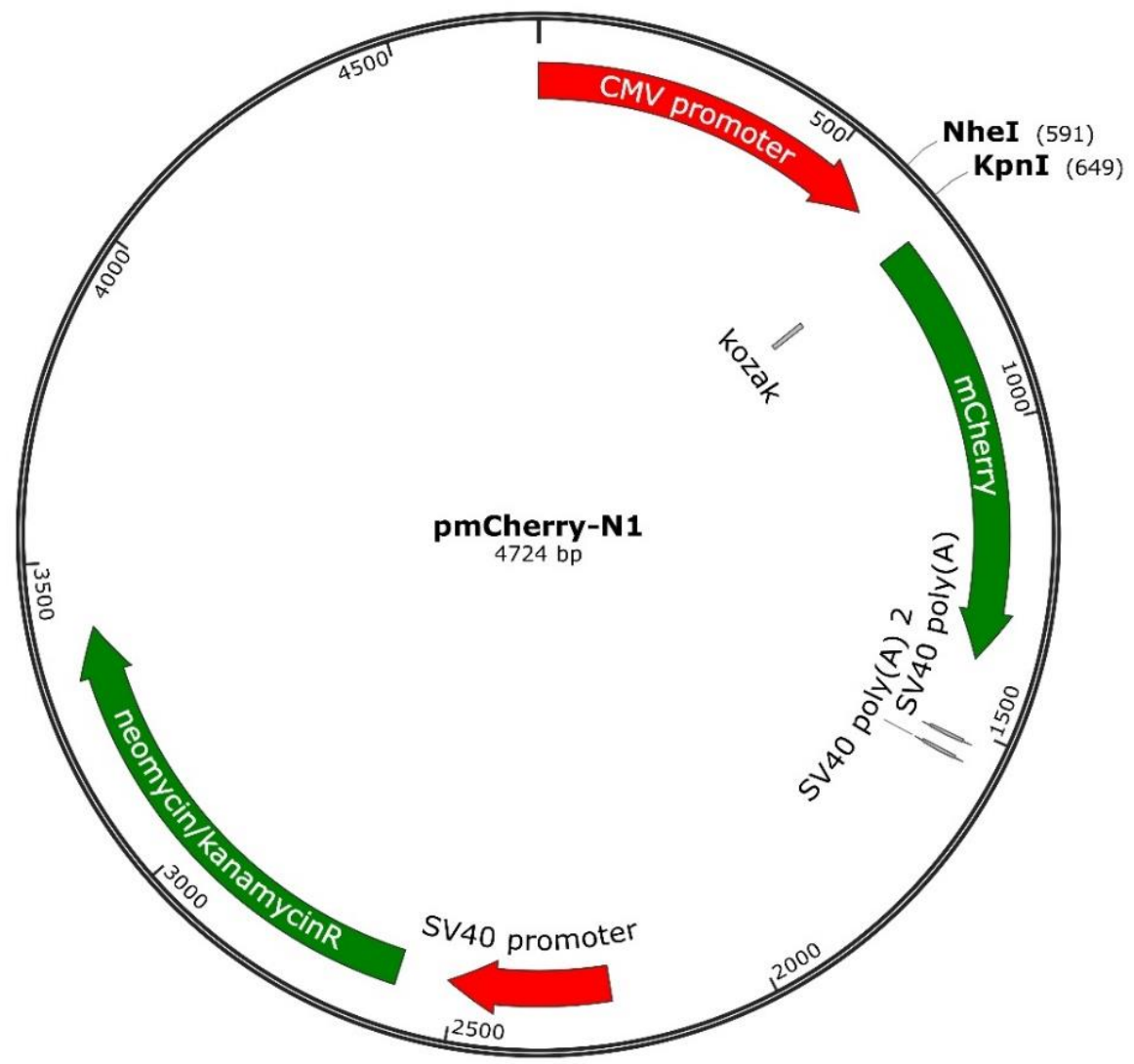

Figure 8-27: Plasmid map of pmCherry-N1 backbone vector.

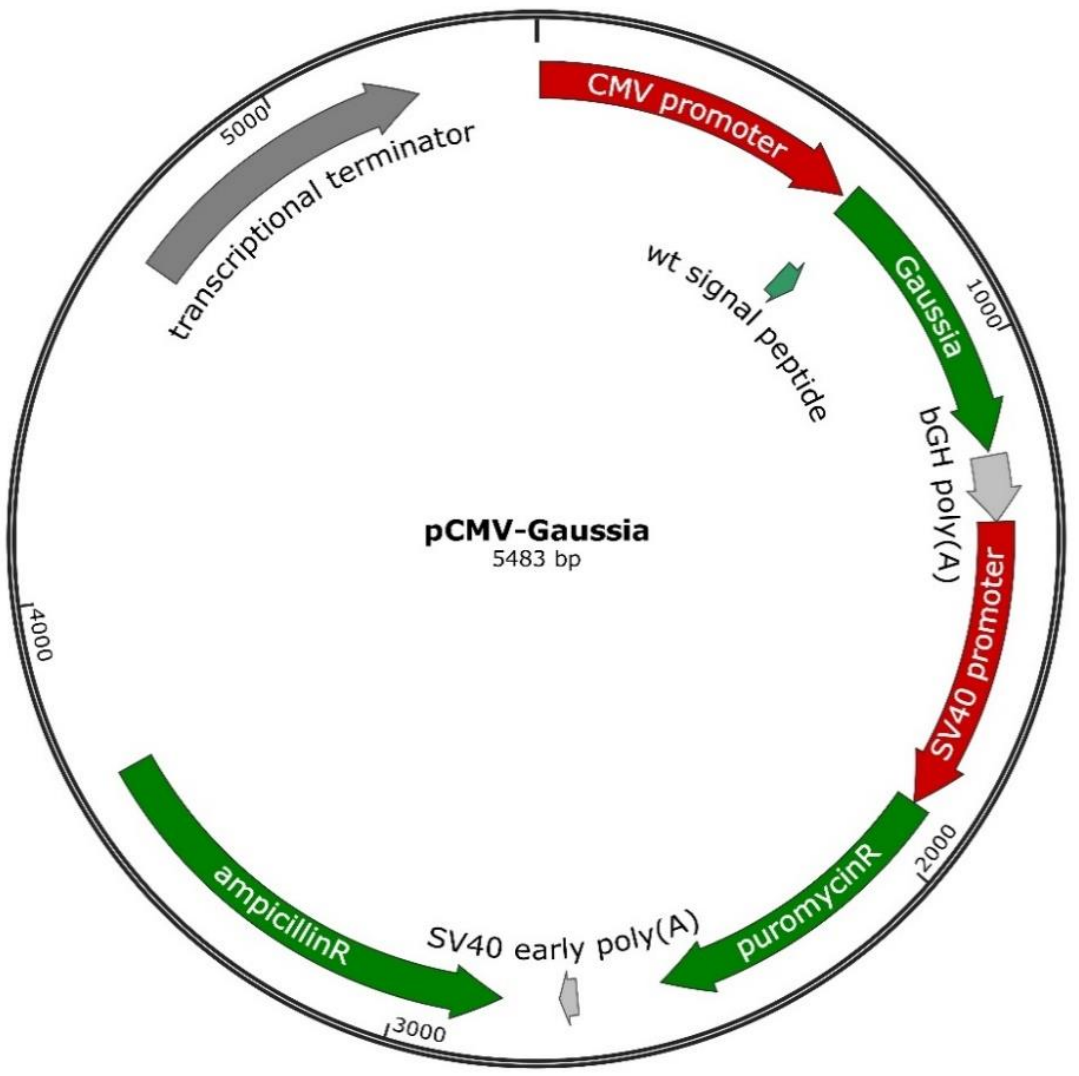

Figure 8-28: Plasmid map of pCMV-Gaussia vector. 


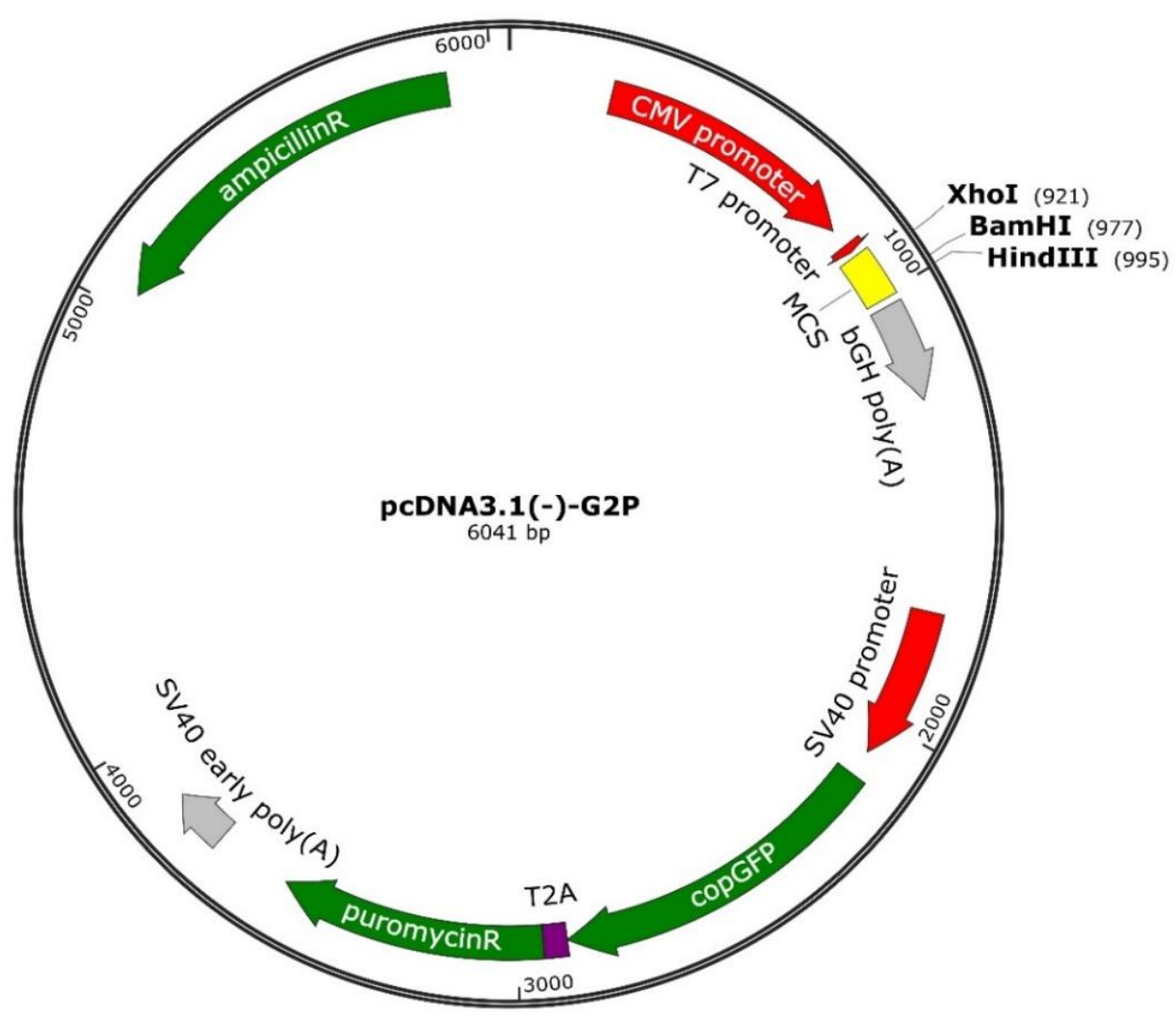

Figure 8-29: Plasmid map of pcDNA3.1(-)-G2P vector.

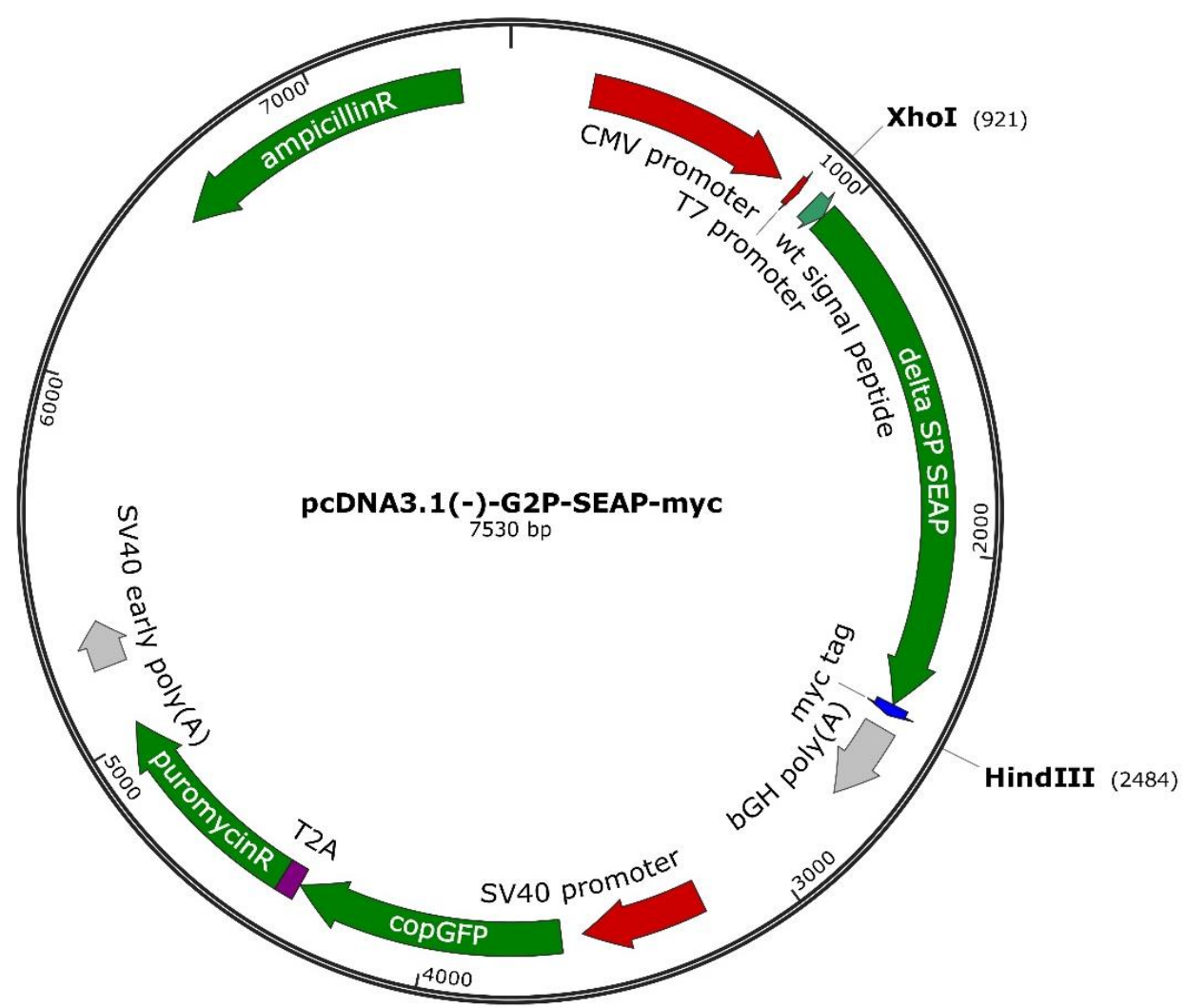

Figure 8-30: Plasmid map of pcDNA3.1(-)-G2P-SEAP-myc vector. 


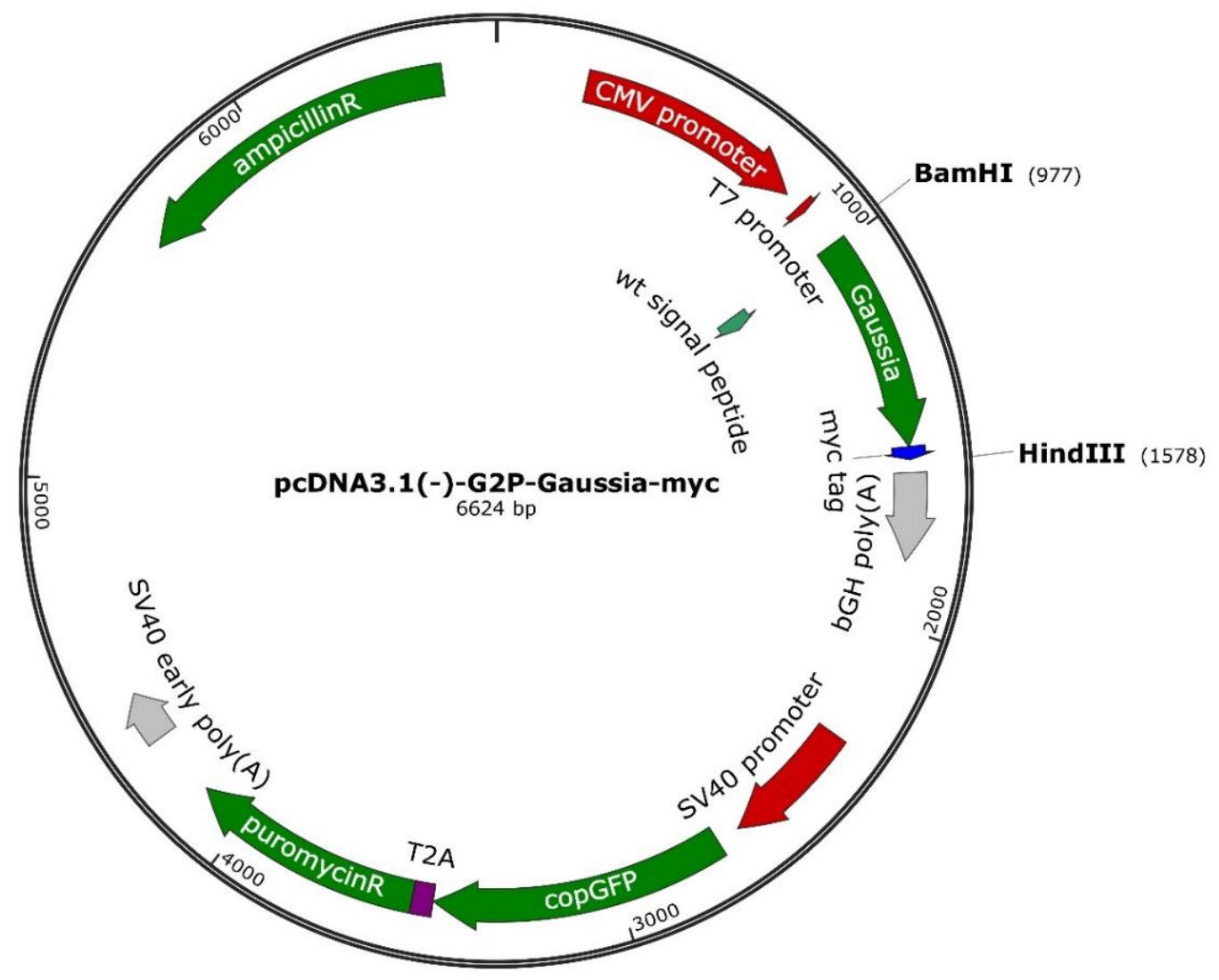

Figure 8-31: Plasmid map of pcDNA3.1(-)-G2P-GLuc-myc vector.

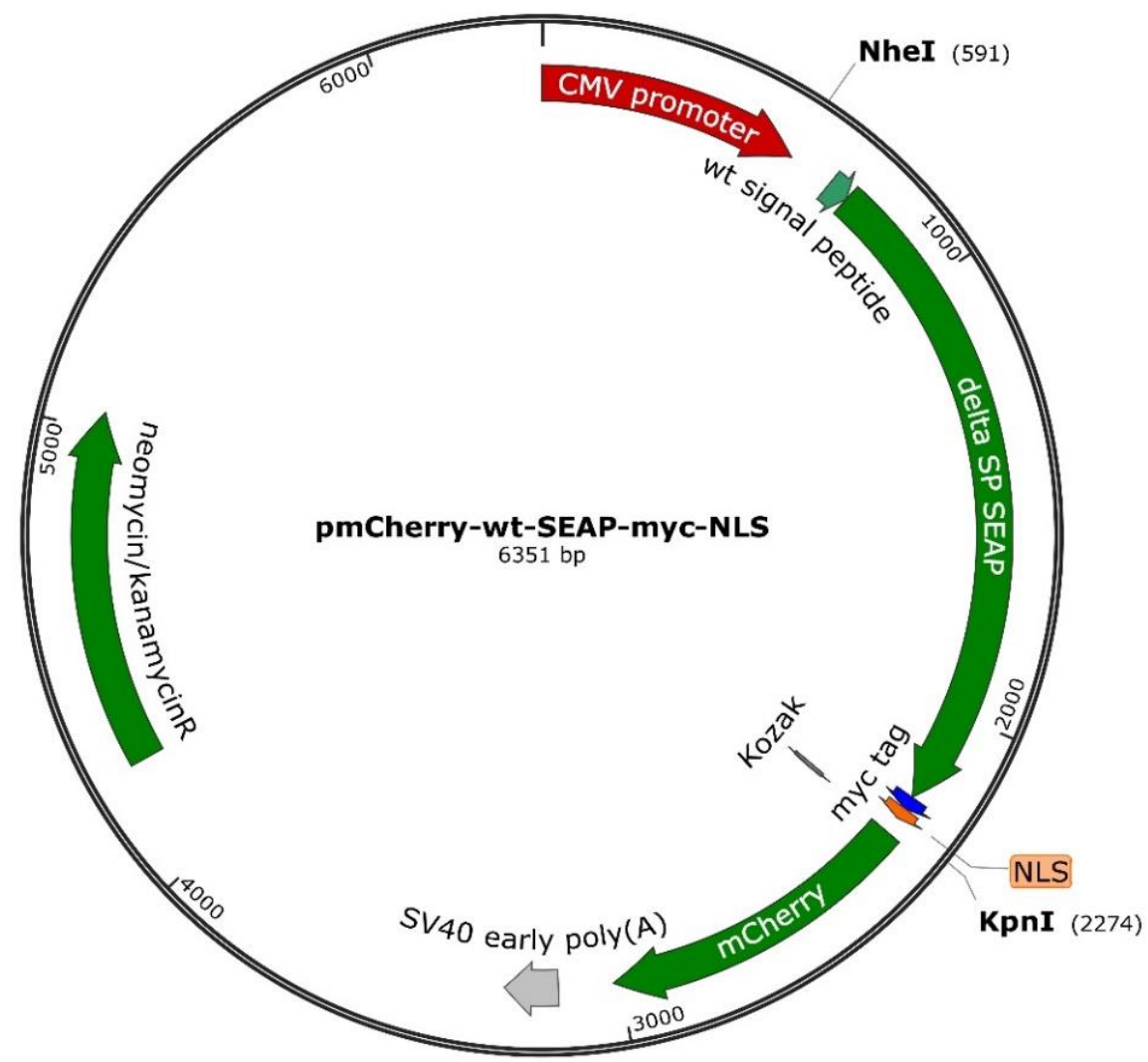

Figure 8-32: Plasmid map of pmCherry-wt-SEAP-myc-NLS vector. 


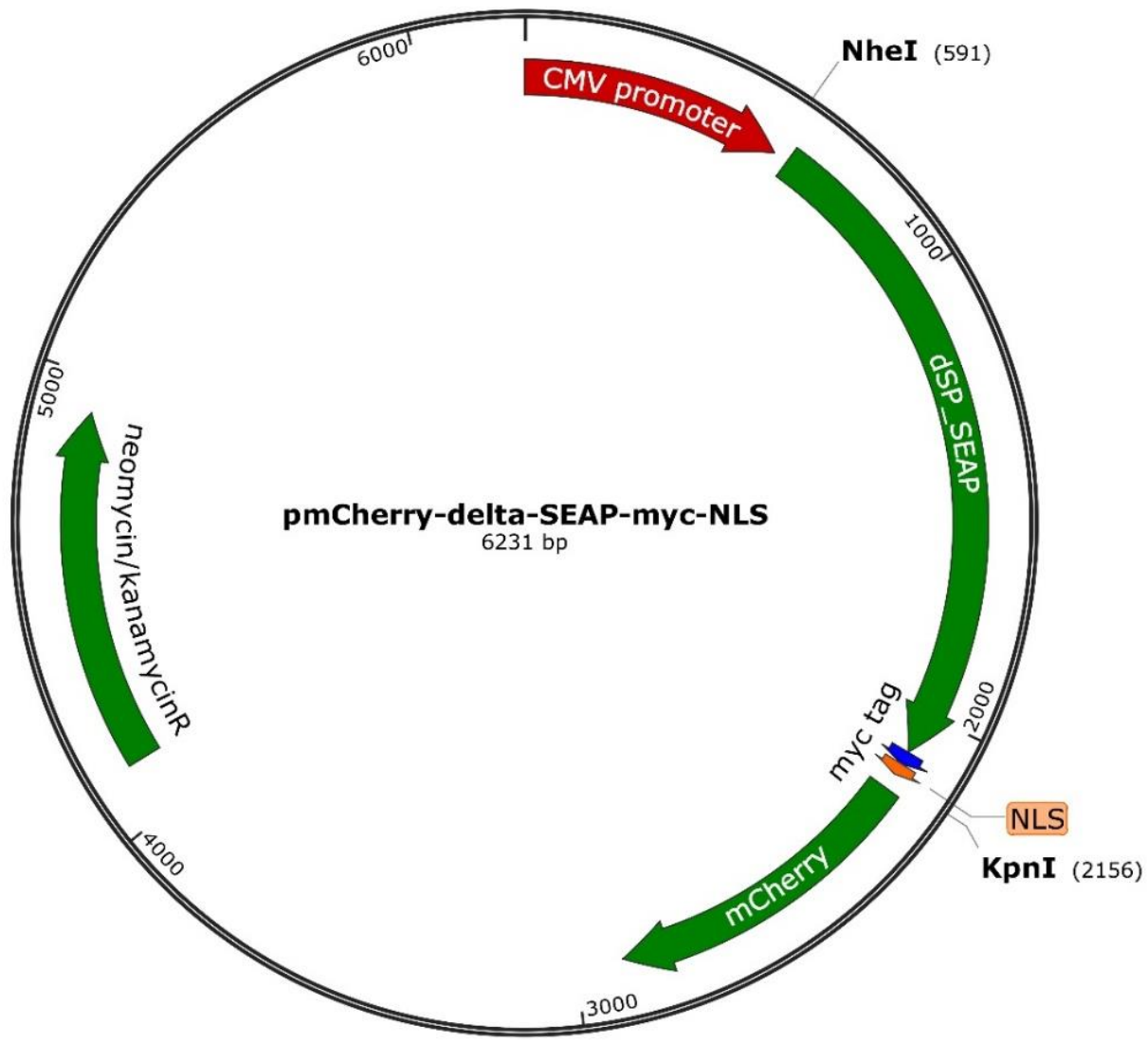

Figure 8-33: Plasmid map of pmCherry- $\triangle$ SEAP-myc-NLS vector.

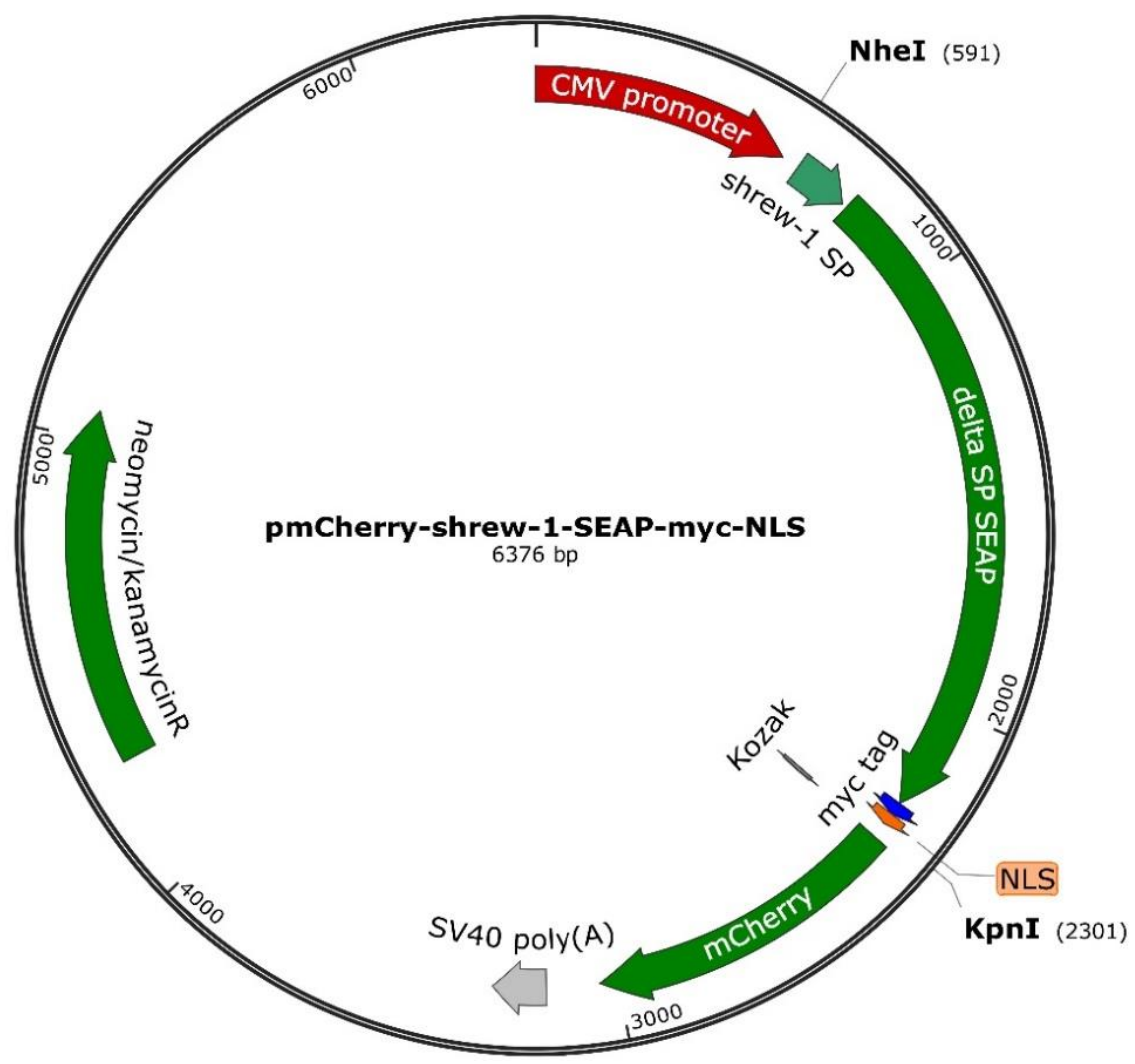

Figure 8-34: Plasmid map of pmCherry-shrew-1-SEAP-myc-NLS vector. 


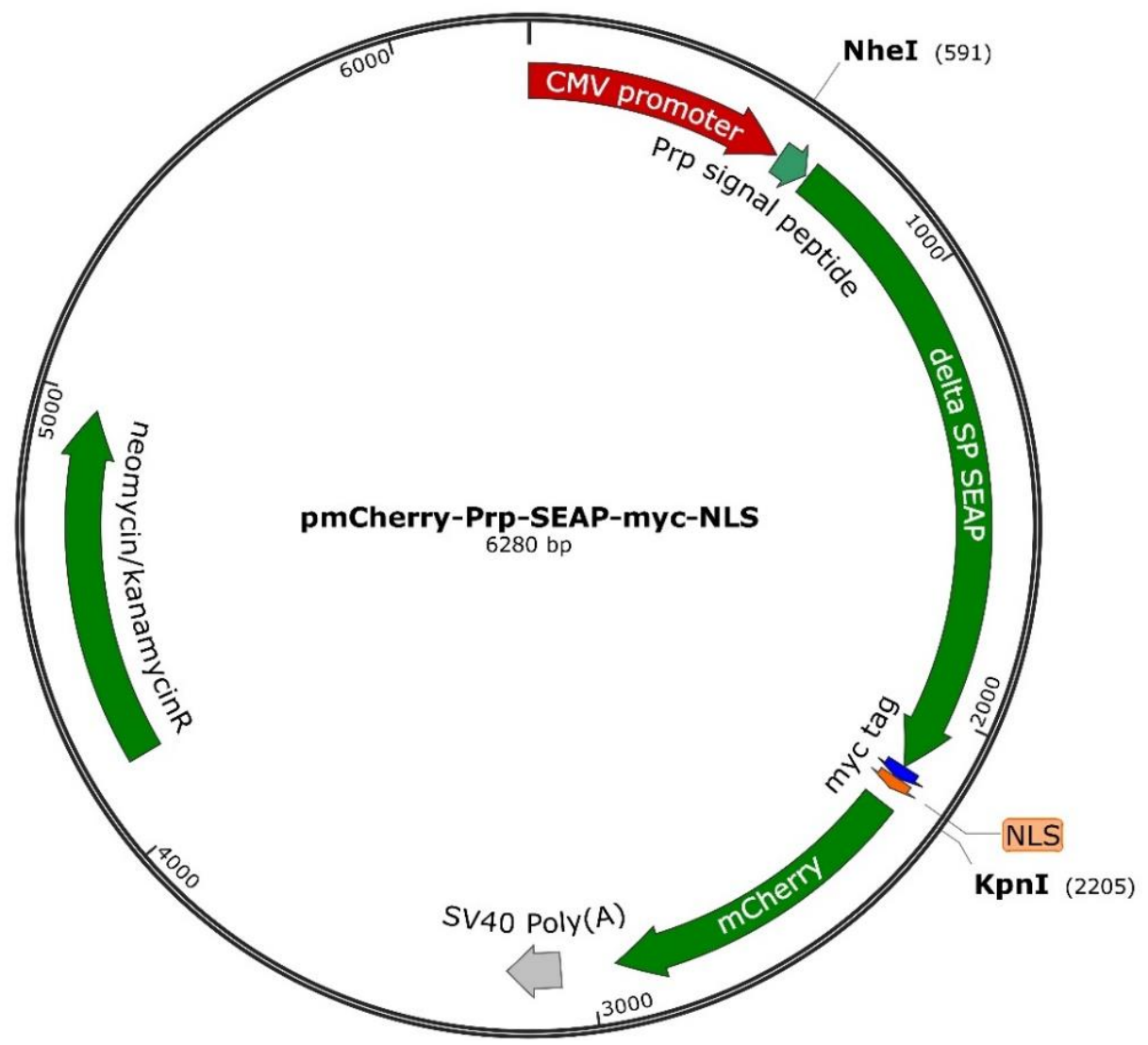

Figure 8-35: Plasmid map of pmCherry-Prp-SEAP-myc-NLS vector.

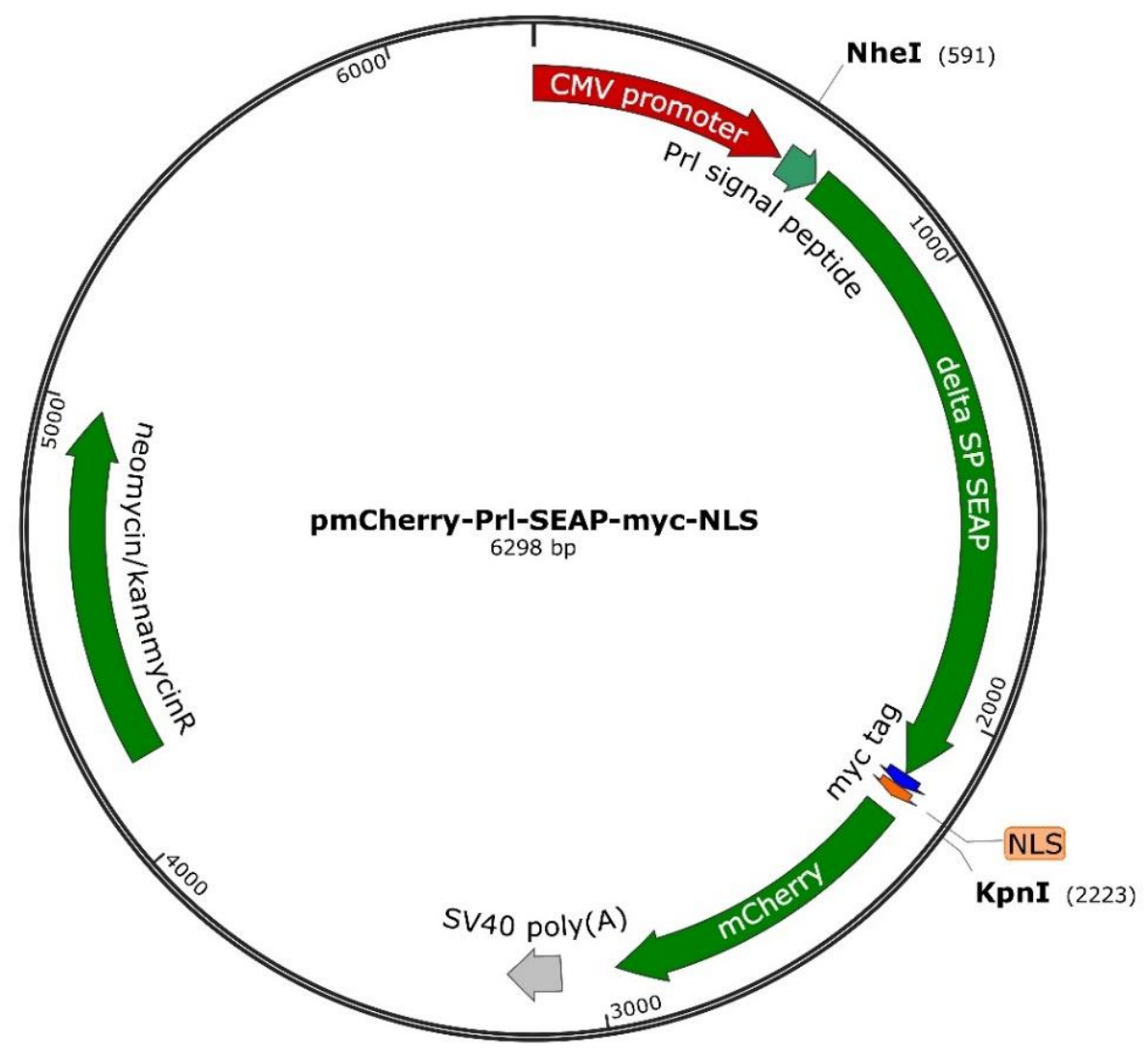

Figure 8-36: Plasmid map of pmCherry-PrI-SEAP-myc-NLS vector. 


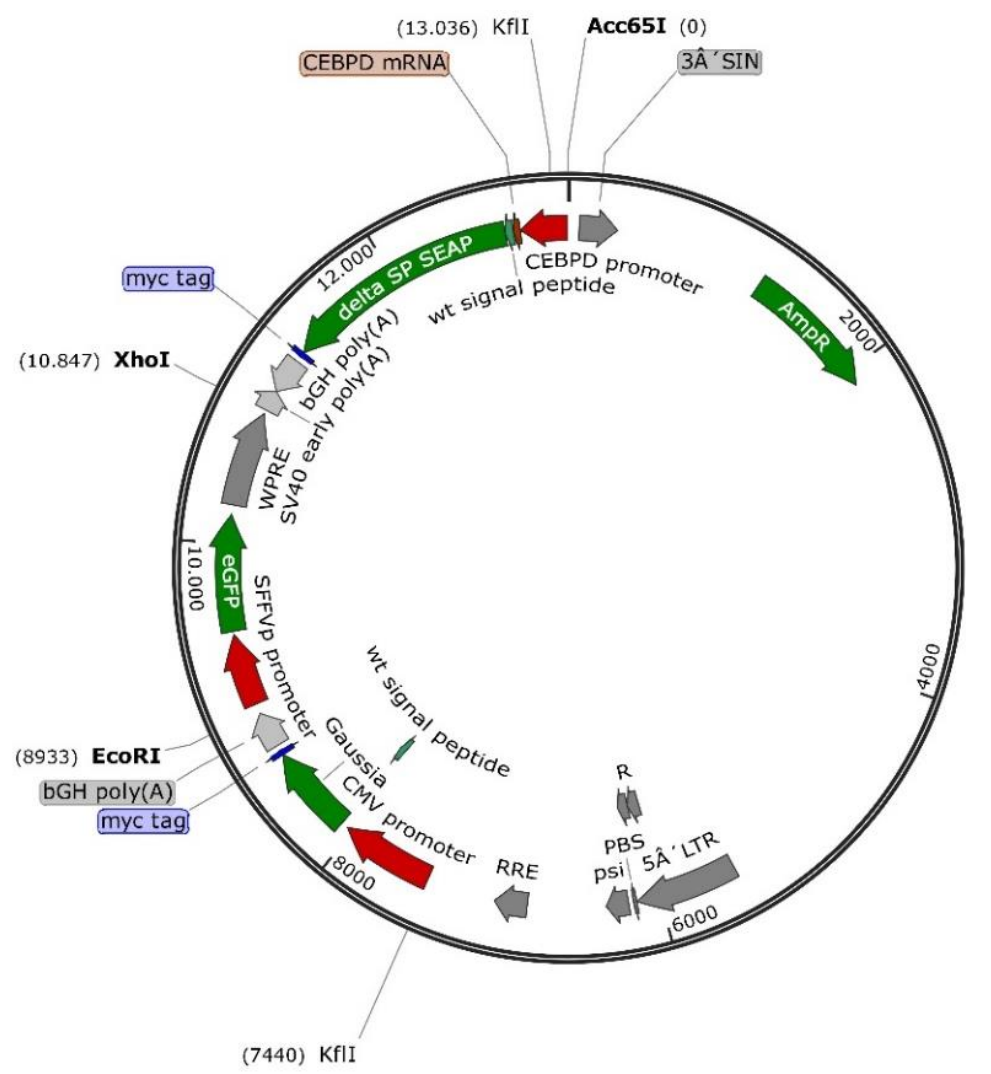

pSEW-eGFP-CMV-GLUC-myc-CEBPD-SEAP-myc

$$
13.134 \mathrm{bp}
$$

Figure 8-37: Plasmid map of pSEW-eGFP-CMV-GLuc-myc-CEBPD-SEAP-myc vector.

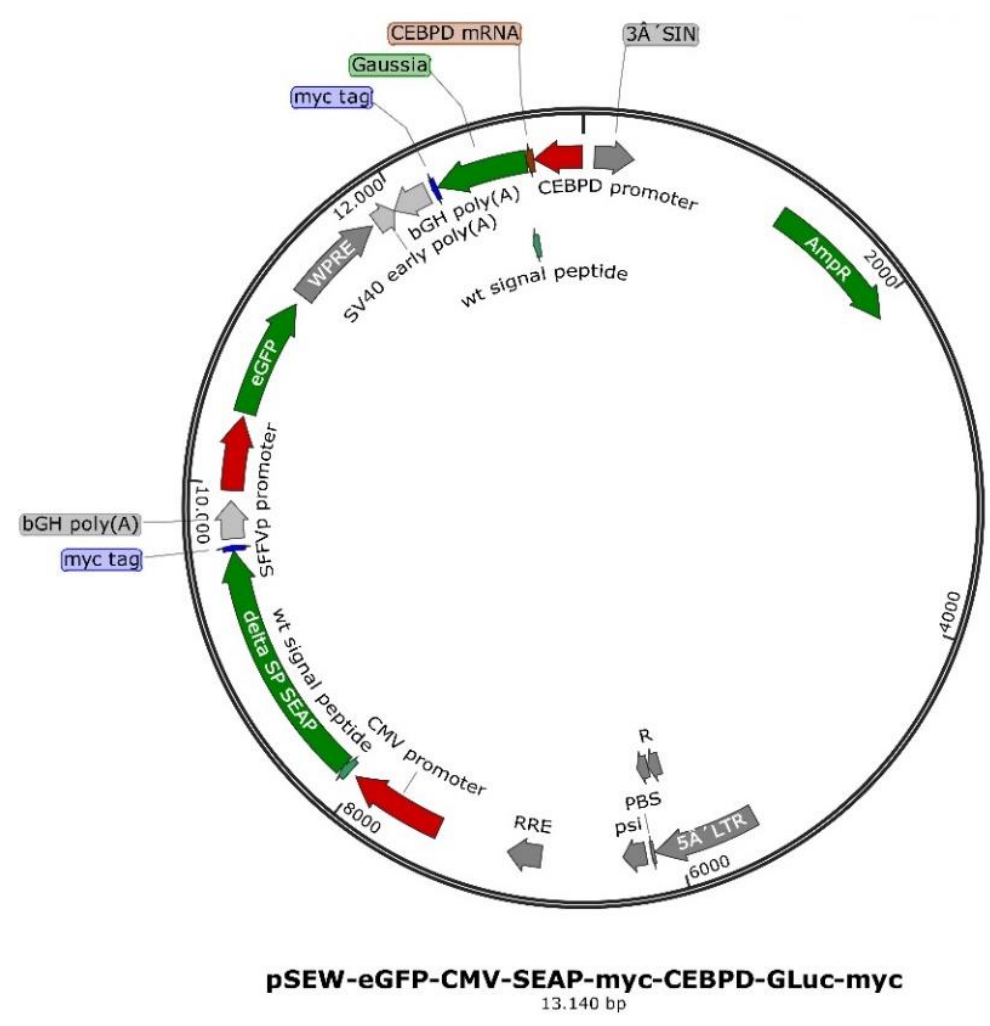

Figure 8-38: Plasmid map of pSEW-eGFP-CMV-SEAP-myc-CEBPD-GLuc-myc vector. 


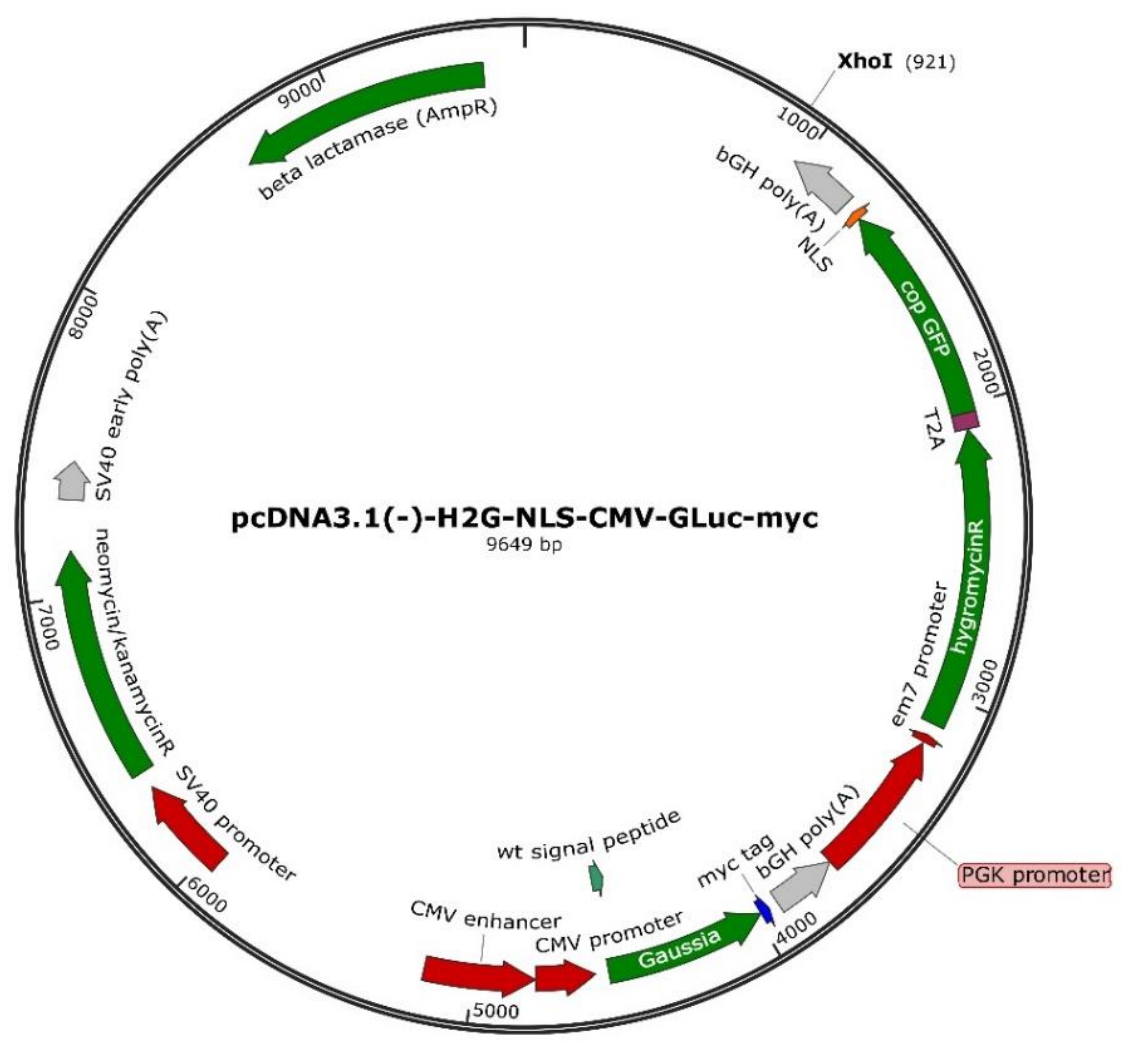

Figure 8-39: Plasmid map of pcDNA3.1(-)-H2G-NLS-CMV-GLuc-myc vector.

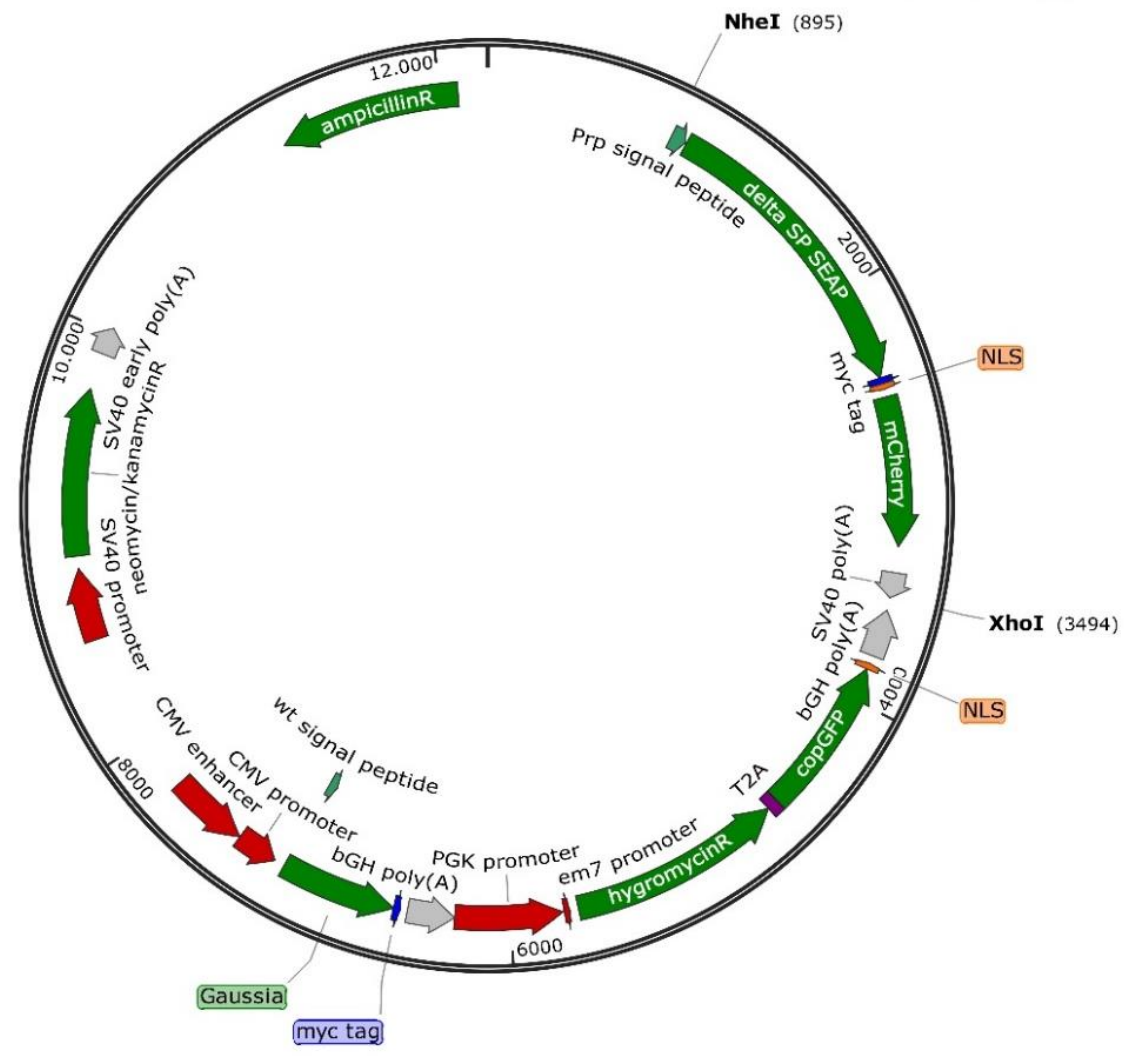

pCDNA3.1(-)-Prp-SEAP-myc-NLS-H2G-NLS-CMV-GLuc-myc

Figure 8-40: Plasmid map of pcDNA3.1(-)-Prp-SEAP-myc-NLS-H2G-NLS-CMV-GLuc-myc vector. 


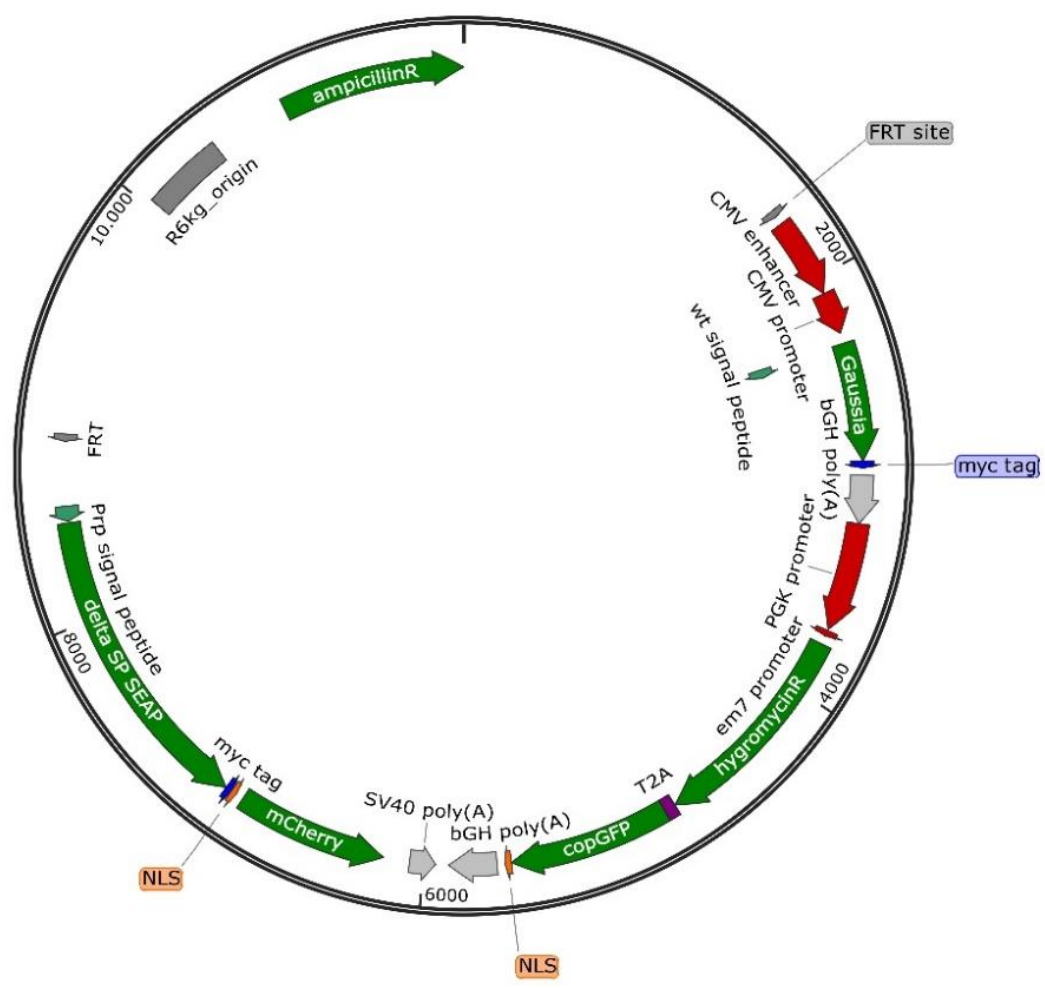

pR6k-Prp-SEAP-myc-NLS-H2G-NLS-CMV-GLuc-myc

Figure 8-41: Plasmid map of pR6k-Prp-SEAP-myc-NLS-H2G-NLS-CMV-GLuc-myc vector.

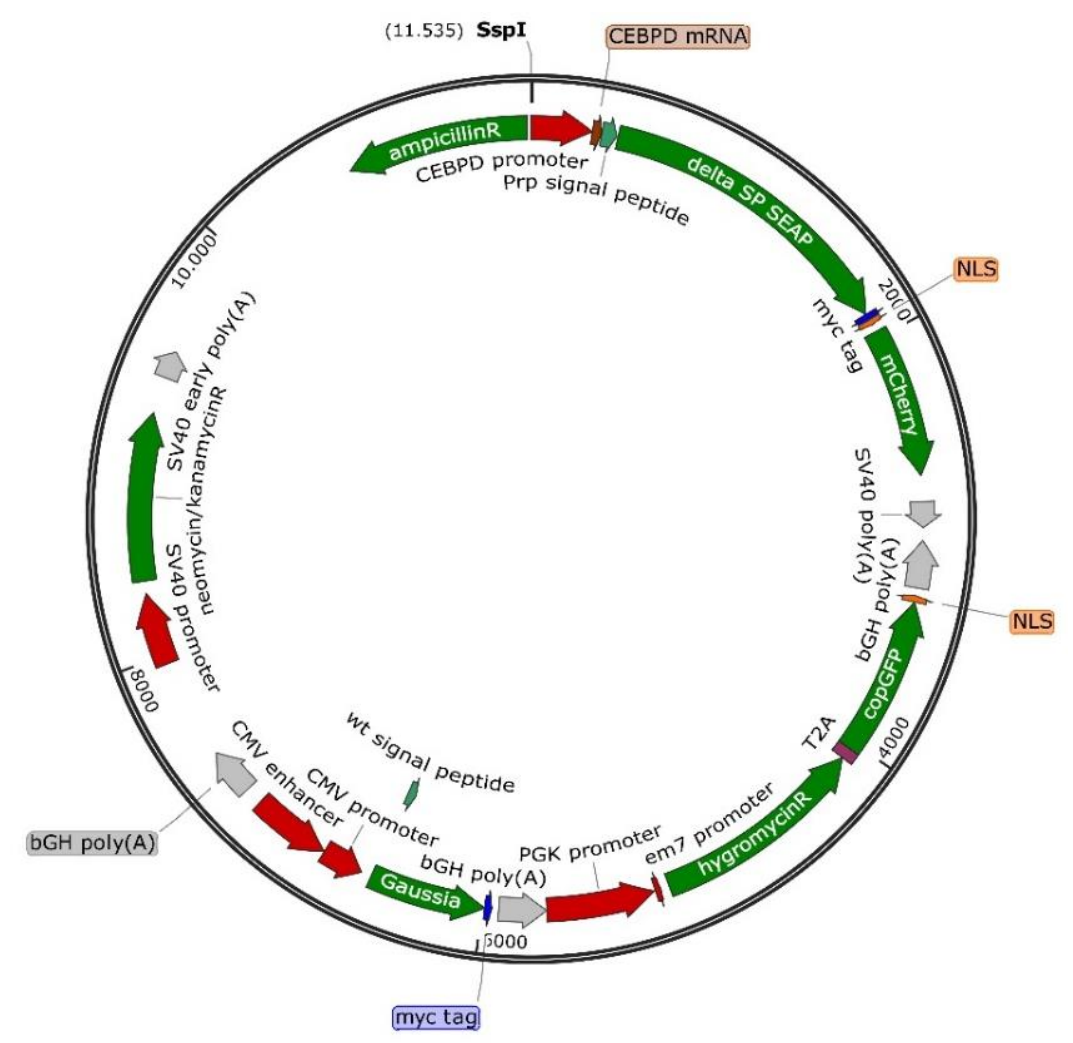

PCDNA3.1(-)-CEBPD-Prp-SEAP-myc-NLS-H2G-NLS-CMV-GLuc-myc

Figure 8-42: Plasmid map of pcDNA3.1(-)-CEBPD-Prp-SEAP-myc-NLS-H2G-NLS-CMV-GLuc-myc vector 


\section{Erklärung}

\section{Schriftliche Erklärung}

Ich erkläre ehrenwörtlich, dass ich die dem Fachbereich Medizin der Johann Wolfgang Goethe-Universität Frankfurt am Main zur Promotionsprüfung eingereichte Dissertation mit dem Titel

Screening for CEBPD-Modulating Compounds Using a THP-1-Derived Reporter Cell Line in the Context of Rheumatoid Arthritis

in dem Institut für Klinische Pharmakologie unter Betreuung und Anleitung von Prof. Dr. Dr. Gerd Geißlinger mit Unterstützung durch Dr. Eduard Resch ohne sonstige Hilfe selbst durchgeführt und bei der Abfassung der Arbeit keine anderen als die in der Dissertation angeführten Hilfsmittel benutzt habe. Darüber hinaus versichere ich, nicht die Hilfe einer kommerziellen Promotionsvermittlung in Anspruch genommen zu haben.

Ich habe bisher an keiner in- oder ausländischen Universität ein Gesuch um Zulassung zur Promotion eingereicht*. Die vorliegende Arbeit wurde bisher nicht als Dissertation eingereicht.

(Ort, Datum)

(Unterschrift) 